University of Warwick institutional repository: http://go.warwick.ac.uk/wrap

A Thesis Submitted for the Degree of PhD at the University of Warwick

http://go.warwick.ac.uk/wrap/73129

This thesis is made available online and is protected by original copyright.

Please scroll down to view the document itself.

Please refer to the repository record for this item for information to help you to cite it. Our policy information is available from the repository home page. 


\section{A Metrological \\ Scanning force microscope}

Ying $\mathrm{Xu}$

(M.Sc, B.Sc)

Submitted for the degree of Doctor of Philosophy to the Higher Degrees Commttee

University of Warwick

Centre for Nanotechnology and Microengineering

Department of Engineering

University of Warwick, Coventry; U.K.

March 1995 


\section{Boston Spa, Wetherby}

West Yorkshire, LS23 7BQ

www.bl.uk

\section{BEST COPY AVAILABLE.}

\section{VARIABLE PRINT QUALITY}




\section{Abstract}

In last decade, there has been a tremendous progress in scanning probe microscopies, some of which have achieved atomic resolution. However, there still exist some problems which have to be solved before the instrument can be used as a metrological measurement tool. The object of the project introduced in this thesis was to develop a scanning force microscope of metrological capability with the aim of making significant improvement in scanning force microscopy from the viewpoint of instrumentation.

A capacitance based force probe has been studied theoretically and experimentally with the main concern being its dynamic properties, characterized by squeeze air film damping, which are believed to have direct effects on the fidelity of measurement. The optimization of design is investigated so as to achieve the results of both high displacement sensitivity and force sensitivity.

An $x-y$ scanning stage has been designed and built, which consists of a two axis linear flexure system of motion amplifying mode machined from a single aluminium alloy block. The stage is driven by two piezo actuators with two capacitance sensors monitoring the actual position of the platform to form a closed loop control system. The design strategy is introduced and the performances and characteristics of two commonly used types of flexure translation mechanisms, leaf spring and notch hinge spring system, are analyzed. The finite element analysis method is employed in the analysis and design of translation mechanism.

Finally, a metrological scanning force microscope has been constructed, combining a constant force probe system, an $x-y$ scanning stage and a 3D coarse positioning mechanism into a metrological system. The performance of the instrument system has been systematically evaluated and its measuring capability investigated on the specimens of various properties and features. The results from this first prototype of the instrument demonstrated a subnanometer resolution with comparable stability and repeatability in all three axes. 


\section{Acknowledgment}

I would like to take this opportunity to express my sincere gratitude to Dr S.T. Smith, my supervisor, for his continual support and encoüragement, without which this project would not have been possible. His effort in improving my academic writic:g skill is also appreciated.

My sincere thanks to Professor D.K. Bowen, my supervisor at the later stage of my Ph.D course, for his effective supervision and help in the process of writing up the thesis. I like his dynamic working style.

Thanks also go to Dr. D.G. Chetwynd for his help and useful advices on my project, and to Dr. X. Liu for her help in my laboratory work.

Thanks to the technicians at the Centre for Nanotechnology and Microengineering: Steve, Dave, Rhod and Frank for their careful craftsmanship and friendship.

The Queensgate Instruments Ltd. is to be thanked for the cooperation of building the precision X-Y scanning stage and providing experiment facilities.

Finally, I am very grateful to Chinese government, British government and Sir. Y.K. Pac for a Sino-British Friendship Scholarship which supported my study in U.K. for last three years. 


\section{Declaration}

This thesis is presented in accordance with the regulations for the degree of Doctor of philosophy. It has been composed by myself and has not been submitted in any previous application for a higher degree. The work described has been undertaken by myself. 


\section{Contents}

1 Introduction 1

1.1 Surface metrology in engineering . . . . . . . . . . . . . 1

1.2 Instrumentation for surface metrology . . . . . . . . . 2

1.2.1 Stylus instruments . . . . . . . . . . . 3

1.2 .2 Optical methods $\ldots \ldots \ldots \ldots \ldots \ldots$

1.2.3 Modern scanning microscopies . . . . . . . . . . 9

2 Development of scanning probe microscopes 17

2.1 Scanning probe microscopy . . . . . . . . . . . 17

2.1.1 Scanning tunneling microscopy $\ldots \ldots \ldots \ldots 17$

2.1.2 Scanning force microscopy . . . . . . . . . . . . 19

2.1.3 Scanning near-field optical microscopy . . . . . . . . 23

2.1.4 Scanning capacitance microscopy . . . . . . . . . . 23

2.1.5 Scanning thermal microscopy . . . . . . . . . . . 24

2.2 SFM transduction methods $\ldots \ldots \ldots \ldots \ldots \ldots$

2.2.1 Tunneling detection system $\ldots \ldots \ldots \ldots \ldots$

2.2.2 Capacitance detection system $\ldots \ldots \ldots \ldots 27$

2.2.3 Optical detection system . . . . . . . . . . . 31

2.2.3.1 Optical beam deflection . . . . . . . . . 31

2.2.3.2 Laser-diode feedback detection . . . . . . . 33

2.2.3.3 Interferometer detection . . . . . . . . . . 33

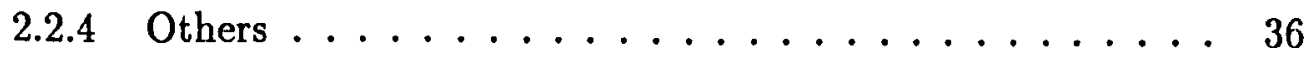


2.3 Scanning and coarse positioning mechanism $\ldots \ldots \ldots \ldots$

2.3.1 Scanning mechanism . . . . . . . . . . . . . 37

2.3.1.1 Piezoelectric tripod . . . . . . . . . . . 38

2.3.1.2 Tube scanner . . . . . . . . . 38

2.3.1.3 Bimorph scanner . . . . . . . . . . . . 40

2.3.1.4 Piezo-flexure stage . . . . . . . . . . 41

2.3.2 Coarse positioning mechanism . . . . . . . . . 42

2.3.2.1 Inchworm . . . . . . . . . . . 43

2.3.2.2 Piezo-walker . . . . . . . . . . 43

2.3.2.3 Magnetic walker . . . . . . . . . 45

2.3.2.4 Inertial glider . . . . . . . . . . . 46

2.3.2.5 Screw-driven mechanism . . . . . . . . 47

3 Force probe system $\quad 58$

3.1 Introduction . . . . . . . . . . . . . 58

3.2 Effects of damping on surface measurement with stylus method - 60

3.3 Dynamic characteristics of the force probe: theoretical analysis . . 63

3.4 Experimental assessment of the theoretical model . . . . . . . 73

3.5 Parameter design . . . . . . . . . . . . . . 80

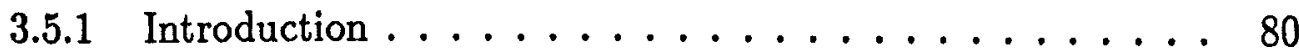

3.5.2 Mathematical model . . . . . . . . . . . . 81

3.5.3 Determination of optimal parameters . . . . . . . 83

3.6 Fabrication of the force probe $\ldots \ldots \ldots \ldots \ldots$

3.7 Force probe system $\ldots \ldots \ldots \ldots \ldots \ldots \ldots$

3.7 .1 Linearity . . . . . . . . . . . . . . 92

3.7.2 Constant force probe system . . . . . . . . . . . . 96

3.8 Conclusion . . . . . . . . . . . . . . . . 100

4 Precision X-Y stage 103

4.1 Introduction . . . . . . . . . . . . . 103 
4.2 Flexure translation mechanisms . . . . . . . . . . . . 104

4.2 .1 Stiffness . . . . . . . . . . . . . 105

4.2 .2 Deflection range . . . . . . . . . . . . . 108

4.2.3 Parasitic deflection errors $\ldots \ldots \ldots \ldots \ldots$

4.2.3.1 Effect of driving force . . . . . . . . 110

4.2.3.2 Effect of manufacture . . . . . . . . 115

4.2.4 Compound rectilinear mechanisms . . . . . . . . . 118

4.2.5 Two dimensional rectilinear mechanisms . . . . . . . . 119

4.3 Design of precision X-Y scanning stage for SPM . . . . . . . 124

4.3 .1 Introduction . . . . . . . . . . . . . . . 124

4.3.2 Description of the X-Y stage . . . . . . . . . . . . 124

4.3.3 Some problems in the design . . . . . . . . . . 127

4.3.4 Dynamic modelling . . . . . . . . . . . . . 130

4.3.5 Experimental assessment of motion errors . . . . . . 132

4.3.6 Finite element analysis . . . . . . . . . . . 135

4.3.6.1 Geometrical model building and mesh generation 135

4.3.6.2 Boundary condition definition . . . . . . . 136

4.3.6.3 Static deflections . . . . . . . . . . 138

4.3.6.4 Dynamic solution . . . . . . . . . . . . 146

4.4 Discussion and conclusion $\ldots \ldots \ldots \ldots \ldots \ldots$

5 Metrological SFM system 153

5.1 Introduction . . . . . . . . . . . . . 153

5.2 Principle of operation . . . . . . . . . . . . . 154

5.3 Some design details . . . . . . . . . . . . . 158

5.3.1 X-Y axis coarse positioning mechanism . . . . . 158

5.3.2 Probe coarse approach mechanism . . . . . . . . . 161

5.3.3 Measurement loop and material selection . . . . . . . 162

5.3.4 Electronic specification . . . . . . . . . . . 163

5.4 Performance evaluation . . . . . . . . . . . 166 
5.4.1 Instrument calibration $\ldots \ldots \ldots \ldots 6$

5.4 .2 Noise level . . . . . . . . . . . . . . . . . . 171

5.4 .3 Stability and repeatability . . . . . . . . . 174

6 Surface measurement $\quad 183$

6.1 Surface topography . . . . . . . . . . . . . . 183

6.2 Surface roughness measurement . . . . . . . . . . . 192

6.2 .1 Introduction . . . . . . . . . . . . . . . 192

6.2 .2 Instrumentation . . . . . . . . . . . . 193

6.2.3 Experiment process and results . . . . . . . 195

6.2 .4 Discussion . . . . . . . . . . . . . . . 199

6.3 Discussion and conclusion $\ldots \ldots \ldots \ldots \ldots$

7 General discussion and conclusion $\quad 204$

7.1 Metrological issues . . . . . . . . . . . . . . . . 204

7.2 What is measured . . . . . . . . . . . 206

7.3 Further improvement on X-Y stage . . . . . . . . . 208

7.4 Conclusion . . . . . . . . . . . . . . . 210

A Program for force probe design $\quad 212$

B Reaction force $R_{x} \quad 214$ 


\section{List of Tables}

3.1 The calculated values of $c h^{3} / \mu$ vs $l \& b \quad \ldots \ldots \ldots \ldots \ldots 72$

3.2 The comparison of the results of experiment and calculation . . 76

3.3 Damping ratio errors caused by parameter errors . . . . . 80

3.4 The parameters versus properties of the probe $\left(h_{0}=4,5 \mu m\right) \quad \ldots 85$

3.5 The parameters versus properties of the probe $\left(h_{0}=6,8 \mu m\right) \quad \ldots 86$

3.6 The parameters versus properties of the probe $\left(h_{0}=10 \mu \mathrm{m}\right) \quad \ldots 87$

3.7 The nonlinear errors of the capacitance probe $\ldots \ldots \ldots$. . . 95

4.1 Parasitic pitching errors caused by driving forces (from mathematic models) . . . . . . . . . . . . . . . 112

4.2 Parasitic pitching errors caused by driving forces (from FEA models)112

4.3 Rotation error of a simple flexure caused by non-parallel arrangement of parallel hinges . . . . . . . . . . . . . 118

4.4 Measured results for angular errors of the $x-y$ stage $\ldots \ldots \ldots 134$

4.5 The parameters chosen for element size setting . . . . . . . 136

4.6 The results of static solution of FEA $\ldots \ldots \ldots \ldots$

6.1 Results of the measurements by Nanostep, SFM and GIXR . . . 197 


\section{List of Figures}

1.1 Block diagram of relationship between surface measurement, manufacturing and function .............. 2

1.2 Schematic diagram of typical stylus instrument $\ldots \ldots \ldots \ldots$

1.3 Schematic diagram of stylus datum systems $\ldots \ldots \ldots \ldots$

1.4 Optical arrangement of Foucault knife-edge follower . . . . . . 6

1.5 Interferometer for surface metrology. (a) Interference fringes, (b) Fizeau interferometer . . . . . . . . . . . . 8

1.6 Scherratic arrangement of X-ray reflectometry . . . . . . . . 9

1.7 Schematic diagram of an SEM . . . . . . . . . . . 11

2.1 Schametic diagram of the configuration of atomic scanning microscope . . . . . . . . . . . . . . . . 20

2.2 The plot of interatomic force . . . . . . . . . . . 21

2.3 Schematic diagram of tunneling detection system $\ldots \ldots \ldots 26$

2.4 Schematic diagram of capacitance detection system $\ldots \ldots \ldots 28$

2.5 Schematic diagram of electrostatic force balanced force probe . . 29

2.6 Schematic diagram of a high stiff tribological force probe . . . . 30

2.7 Schematic diagram of optical beam deflection detection system . . 32

2.8 Schematic diagram of laser-diode feedback detection system . . . 34

2.9 Schematic diagram of homodyne detection system . . . . . . 35

2.10 Schematic diagram of fiber-optic interferometer detection system . 36

2.11 Schematic diagram of piezoelectric tripod scanning mechanism . . 39

2.12 Scherratic diagram of tube scanner $\ldots \ldots \ldots \ldots \ldots$ 
2.13 Bendiag mode of tube scanner. (a) Simple bending, (b) S-shape bending ..................... . . 40

2.14 Schentatic diagram of bimorphscanner . . . . . . . 41

2.15 Schematic diagram of piezo-flexure stage, (From reference 59) $\ldots 42$

2.16 Operation principle of inchworm. (a) The inchworm is ready for a new step. piezo 1 clamps. (b) Piezo 2 expands. (c) Piezo 3 clamps and Piezo 1 releases. (d) Piezo 2 contracts, and the tube is moving up. . . . . . . . . . . . . . . . . . . 44 44

2.17 Schematic diagram of operation principle of piezo-walker $\ldots \ldots 45$

2.18 Schematic diagram of magnetic walker . . . . . . . . . 46

2.19 Schematic diagram of inertial glider . . . . . . . . 47

2.20 Schematic diagram of a differential spring reduced screw positioner 48

3.1 The force probe $\ldots \ldots \ldots \ldots \ldots \ldots \ldots$

3.2 Schematic diagram of simplified model of general stylus method . 61

3.3 Surface spectrum weighting factors for different damping system

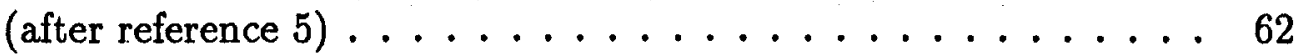

3.4 Modeling parameters of the force probe . . . . . . . . . . 64

3.5 Beam model satisfying the boundary condition of zero flow at the clamped end and ambient pressure at the beam edge . . . . .

3.6 The distribution of the damping pressure along central axis of a cantilever beam, $b=1.5 \mathrm{~mm}, V_{0}=1 \mathrm{~mm} \mathrm{~s}^{-1}, h=15 \mu \mathrm{m} \ldots \ldots$.

3.7 The relationship between damping ratio and gap for a variety of lengths of a glass $\left(E=70 G P_{a}\right)$ cantilever with the dimension $\mathrm{b}=$ $1.5 \mathrm{mrn}, \mathrm{t}=0.1 \mathrm{~mm}$ and tip mass $m_{t}=2 \mathrm{mg} \ldots \ldots \ldots 71$

3.8 The ratationship between damping factor and cantilever geometry 72

3.9 The schematical diagram of the dynamic testing system . . . . . 73

3.10 The measured transfer function of the force probe . . . . . . . 74

3.11 The measured coherence function of the force probe . . . . . . . 74

3.12 The measured transfer function of over damped force probe . . . 75 
3.13 The comparison of the results of experiment and calculation. $\mathrm{x}-$ $\xi_{c}^{*},+-\xi_{c}, o-\xi_{t} \ldots \ldots \ldots \ldots \ldots \ldots$

3.14 Calculated damping forces using different deflection models, $(a)$ results, $(b)$ difference between the two theoretical models $\ldots \ldots 78$

3.15 The comparison of the profiles of two deflection models . . . . 79

3.16 The curve of etching depth against etching time . . . . . . 89

3.17 The diagram of the pattern of aluminium layer deposited on base block . . . . . . . . . . . . . . . . 89

3.18 Schematic diagram of cantilever machining using milling machine 90

3.19 Plot of the output of capacitance $C / K(K=l b \varepsilon)$ versus the deflection of cantilever $\Delta h,\left(h_{\circ}=10 \mu m\right)$, from equation (3.29) $\ldots .93$

3.20 Plot of the linearity error against the capacitance gap thickness $h_{\circ}$ with the same deflection range of $2 \mu m \ldots \ldots \ldots . \ldots 93$

3.21 Plot of the linearity error against the thickness of capacitance gap $h_{o}$ corresponding to relative deflection ranges of $20 \%$ and $30 \%, 94$

3.22 The Curve of the output of probe versus the relative displacement between the probe and surface . . . . . . . . . . 95

3.23 Schematic diagram of constant force servo system of the force probe 97

3.24 The diagram of the constant force profiling system . . . . . . . 97

3.25 Profile of a single diamond turned silicon surface . . . . . . . . 99

4.1 The diagram of simple flexure rectilinear mechanisms . . . . . 106

4.2 The configurations of the flexure springs $\ldots \ldots \ldots \ldots$

4.3 Plot of $\mathrm{Q}$ in equation (4.10) against $R / t \ldots \ldots \ldots \ldots$

4.4 Simple parallel movement of leaf spring system: (a) undeflected, (b) intended parallel deflexion, (c) undesired deflexion . . . . . . 111

4.5 FEA model of leaf spring and its solution. (a) FEA model deformed and undeformed, (b) Pitching curve of moving platform a-b. . . . . . . . . . . . . . . . . . 113 
4.6 FEA model of hinge spring and its solution. (a) FEA model deformed and undeformed, (b) Pitching curve of moving platform a-b. . . . . . . . . . . . . . . . . . . 114

4.7 Deflexion from parallelism of motion caused by manufacturing errors 117

4.8 Schematic diagram of compound rectilinear mechanism . . . . . 120

4.9 Schematic diagram of double compound rectilinear mechanism . 120

4.10 Schematic diagram of a symmetric rectilinear system of threehole/1 wo-web flexure elements . . . . . . . . . . . . . . 121

4.11 Schematic diagram of a symmetric rectilinear system double leaf spring flexures . . . . . . . . . . . . . . . . 121

4.12 Schematic diagram of two dimension rectilinear mechanism . . . 123

4.13 Schematic diagram of configuration of $S 220 x-y$ scanning stage . . 125

4.14 Schematic diagram of translation configuration of the $x-y$ scanning stage . . . . . . . . . . . . . . . . 126

4.15 Schematic diagram of a simple model of motion amplification mechanism for effective stiffness analysis . . . . . . . . . . 129

4.16 The schematic diagram of spring systems on the $x$ and $y$ axes. (a) Model for the $x$ axis, (b) Model for the $y$ axis $\ldots \ldots \ldots \ldots 130$

4.17 The relationship between natural frequency and mass added on the platform.

4.18 The schematic diagram of angular error measurement setup for measuring the yaw and pitch errors of the $x$ axis and the roll error of the $y$ axis . . . . . . . . . . . . . . 134

4.19 Diagram of meshed model for $x-y$ stage (S220) $\ldots \ldots \ldots \ldots 137$

4.20 Diagram of boundary condition for FEA model $\ldots \ldots \ldots$

4.21 Deformed models from FEA static solution. (a) the stage is driven in the $x$ axis, (b) the stage is driven in the $y$ axis $\ldots \ldots \ldots 140$

4.22 The yaw error of platform in the $x$ axis, (Model-A) $\ldots \ldots \ldots 142$

4.23 Distortion curve of nodes along $a-b,($ Model-A) $\ldots \ldots \ldots \ldots 142$ 
4.24 Distortion curve of nodes along $c-d$, (Model-A) . . . . . . . 143

4.25 Distortion curve of nodes along $j-k$, (Model-A) . . . . . . . . 143

4.26 The yaw error of platform in the $x$ axis (Model-B) . . . . . . 144

4.27 Distortion curve of nodes along $a-b$, (Model-B) . . . . . . 144

4.28 Distortion curve of nodes along $c-d$, (Model-B) . . . . . . . 145

4.29 Distortion curve of nodes along $j-k$, (Model-B) . . . . . . 145

4.30 Free vibration mode of the stage, (mode-1) . . . . . . . . 147

4.31 Free vibration mode of the stage, (mode-2) . . . . . . . . 147

4.32 Free vibration mode of the stage, (mode-3) . . . . . . . 148

5.1 Block diagram of SFM system . . . . . . . . . 155

5.2 Photograph of the SFM system . . . . . . . . . 156

5.3 Isometric sketch of the metrological SFM . . . . . . . . 158

5.4 Isometric sketch of the X-Y coarse positioning mechanism $\ldots . .159$

5.5 Section view of $y$ axis locking mechanism . . . . . . . 160

5.6 Diagram of elastic contact mechanism of $Z$ translator carriage . . 161

5.7 Diagram of decoupling mechanism . . . . . . . . . . 162

5.8 Schematic diagram of the measurement loop of the instrument . . 164

5.9 Schematic diagram of $\mathrm{S} 2000$ rack system $\ldots \ldots \ldots \ldots$

5.10 Block diagram of of SM Servo Module . . . . . . . . . 166

5.11 Schematic diagram of calibration system of the $x-y$ stage $\ldots \ldots 168$

5.12 Plot of the calibration curves of the X-Y stage in $x$ axis . . . . 168

5.13 Schematic diagram of calibration set for measurement system in

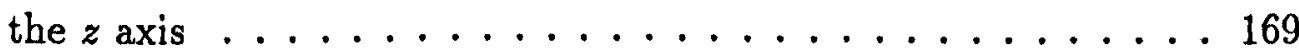

5.14 Plot of the calibration curves of measurement loop in the $z$ axis .170

5.15 Plot of standard step profiles from SFM and Nanostep $\ldots \ldots 170$

5.16 Noise level of the instrument system . . . . . . . . . 171

5.17 Noise level corresponding to sampling processes with various averaging times for each data point. The averaging times for these plots: (a) - zero, (b) -10, (c) -50, (d) $-1000 . \ldots \ldots 173$ 
5.18 Noise level and speed of data acquisition vs. averaging times : . 173

5.19 Noise levels of $x$-y scanning stage in the $x$ axis. (a) - open-loop control state, (b) - closed-loop control state . . . . . . . 175

5.20 Plots of system thermal drift . . . . . . . . . . . 176

5.21 Ramp scan trace for analysis of linearity . . . . . . . . 177

5.22 Plots of two meander pattern scans . . . . . . . . . . 179

5.23 Plot of two optical grating surface profiles obtained from a continuous forward and backward scan ... . . . . . . . 179

6.1 SEM image of normal stylus tip of radius $1 \mu \mathrm{m} \ldots \ldots$. . . . . 184

6.2 SEM image of Berkovich tip of radius $<0.1 \mu \mathrm{m} \ldots \ldots 184$

6.3 Image of optical grating surface. (a) 3D view, (b) Section profile . 187

$6.42 \mathrm{D}$ view of the same grating surface as in Fig.6.3 . . . . . . 188

6.5 SEM image of the same grating surface as in Fig.6.3 . . . . . . 188

6.6 SPM image of standard gratings of 2160 lines $/ \mathrm{mm}$ for SEM cali-

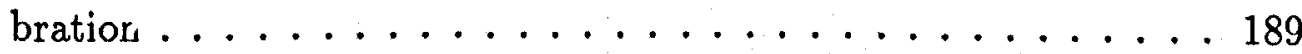

6.7 SPM image of polymer film (D9) . . . . . . . . . . . 189

6.8 SPM image of polymer film (D12) . . . . . . . . . . . 190

6.9 SPM image of polymer film (D2) ............... 190

6.10 SPM image of a well polished Zerodur optical flat . . . . . . . 191

6.11 3D image of electronic components on a silicon chip . . . . . . 191

6.12 Surface topography of $S_{i} / S_{i 0.8} G_{e 0.2} / S_{i}$ heterostructure sample . 192

6.13 Calculated GIXR of a perfect smooth surface and the surfaces with added roughness . . . . . . . . . . . . . . . . 194

6.14 Curve of a specular scan from the GXR1 reflectometer . . . . 198

6.15 SFM image of Surface topography of Zerodur $4 / 1 \ldots 199$

7.1 The interaction forces between a diamond tip and a floating glass cleaned with Acetone . . . . . . . . . . . . . 207

7.2 Schematic diagram of piezo expansion amplifier . . . . . . 209 
B.1 Reaction force model of simple notch hinge rectilinear mechanism 214 


\section{Chapter 1}

\section{Introduction}

\subsection{Surface metrology in engineering}

In engineering terms, surface metrology is usually referred to as the measurement and investigation of geometrical properties of surface, or surface texture, although sometimes other physical, chemical and biological properties may be included. Surface texture describes a surface in terms of the variation in amplitude and spacing. Depending upon the scale of lateral features, surface measurements are classified as form, waviness and roughness when ranging from 'large' to 'small' [1]. Large scale deviations from design shape obviously prevent reasonable operation. Roughness measures small characteristics that also affect the functional performance of engineering components [2]. Applications which are affected by surface finish include tribological regimes of friction, wear and lubrication, as well as optical, electrical and thermal contact properties. Therefore, to investigate or assess these performance related properties of surface, a understanding of surface texture is essential. Besides, the surface topography can also provide the information about manufacturing process or mechanisms used for generation of the surface [3] [4]. As mentioned by Whitehouse [7], the surface is a link between the manufacture of an engineering component and its function. From this perspective, surface measurement is key for both monitoring of manufactur- 
ing process and prediction of component function, as shown in Fig. 1.1. For most conventional machining processes, roughness features will typically contain asperity heights of the order of micrometers. Modern precision manufacturing techniques require that surface features be measured down to the nanometer regime or even smaller. The term 'nanotechnology' was coined by Taniguchi to describe engineering and measurement at such scales [5] [6].

Requirement of surface feature

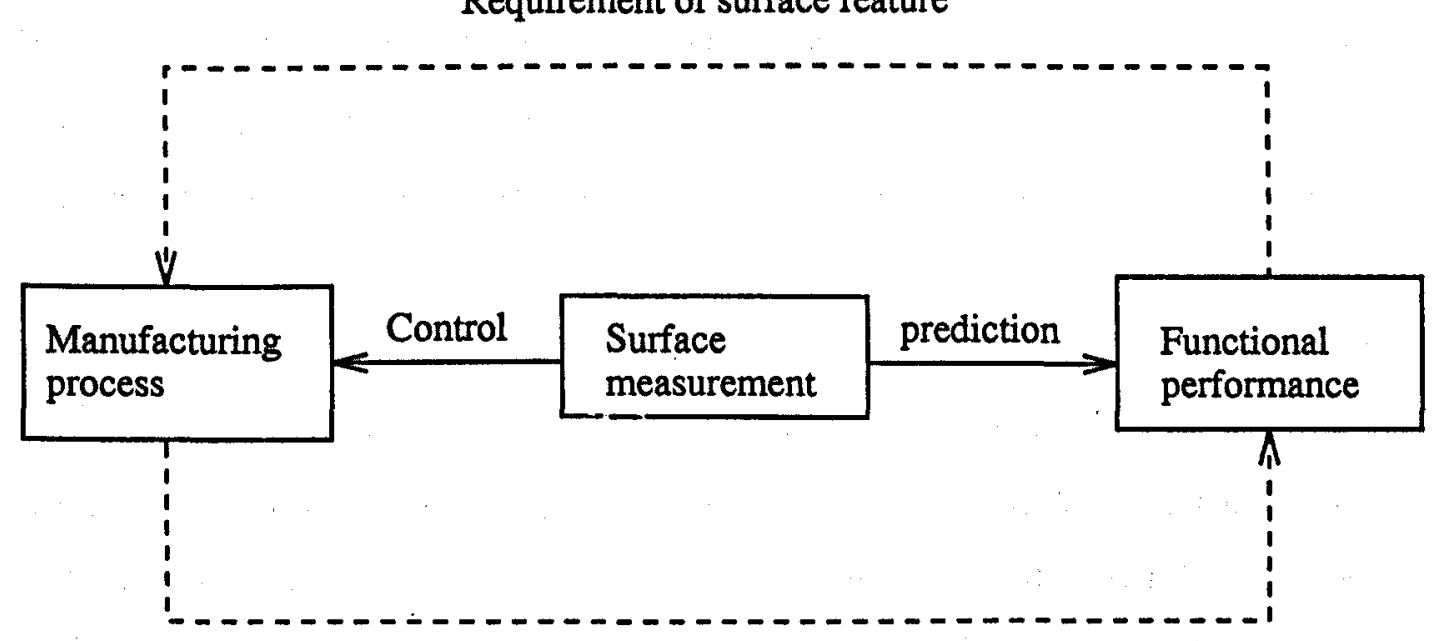

Satisfied surface roughness

Figure 1.1: Block diagram of relationship between surface measurement, manufacturing and function

\subsection{Instrumentation for surface metrology}

To satisfy engineering requirements, a large number of instruments have been developed for the measurement of surface profile or topography. Various physical principles are used to reveal surface geometric features. These instruments can, generally, be categorized into three groups or types: 1) stylus instruments, 2) optical methods, and 3) modern scanning microscopes. They can also be considered as three generations of surface measurement techniques in this sequence. Although there are some overlaps between these techniques in their capabilities, 
none of them can replace the other. Therefore, it is useful to have a general review on these existing instruments before moving to the main topic of the thesis which presents the development of a metrological scanning force microscope which is a particular example of the last category.

\subsubsection{Stylus instruments}

The stylus instrument is one of the most popular instruments used for surface profiling in both the workshop and research laboratory. A typical stylus instrument usually consists of five basic components, 1) a relative displacement transducer, 2) a datum surface, 3) a traverse mechanism, 4) a stylus probe, and 5) a signal and data processing unit. Fig. 1.2 shows a schematic diagram of the instrument. Although the structure details of the instruments may vary considerably, their general arrangements nearly all conform to it [8]. In the system shown in Fig. 1.2, a beam, pivoted on a knife edges, carries the stylus at one end and a transducer at the other. When measuring, the stylus is contacted and drawn by the traverse mechanism across the surface under examination. The irregularities of surface cause the stylus to move vertically, and the motion is sensed by a transducer that monitors the deflection of the beam. The displacement of the stylus relative to a datum surface is then used as a quantitative measurement of the surface profile.

A commonly used stylus transducer is an inductive gauge, LVDT or LVDI, in which a ferromagnetic slug is usually fixed on the beam and located between coils. When the stylus moves and the beam deflects, it causes a change in the mutual inductance in the coils modulating a high frequency carrier signal in proportion to the displacement of the stylus. In addition, some other displacement transducers such as optical interferometers and capacitors are also found in modern instruments [9]. All these transducers are of high sensitivity and capable of carrying out measurements at nanometer levels. Because stylus profiling is a relative measurenient, a properly arranged datum reference is essential. It is usually realized in either of two ways, skid datum or independent datum [10]. The skid 


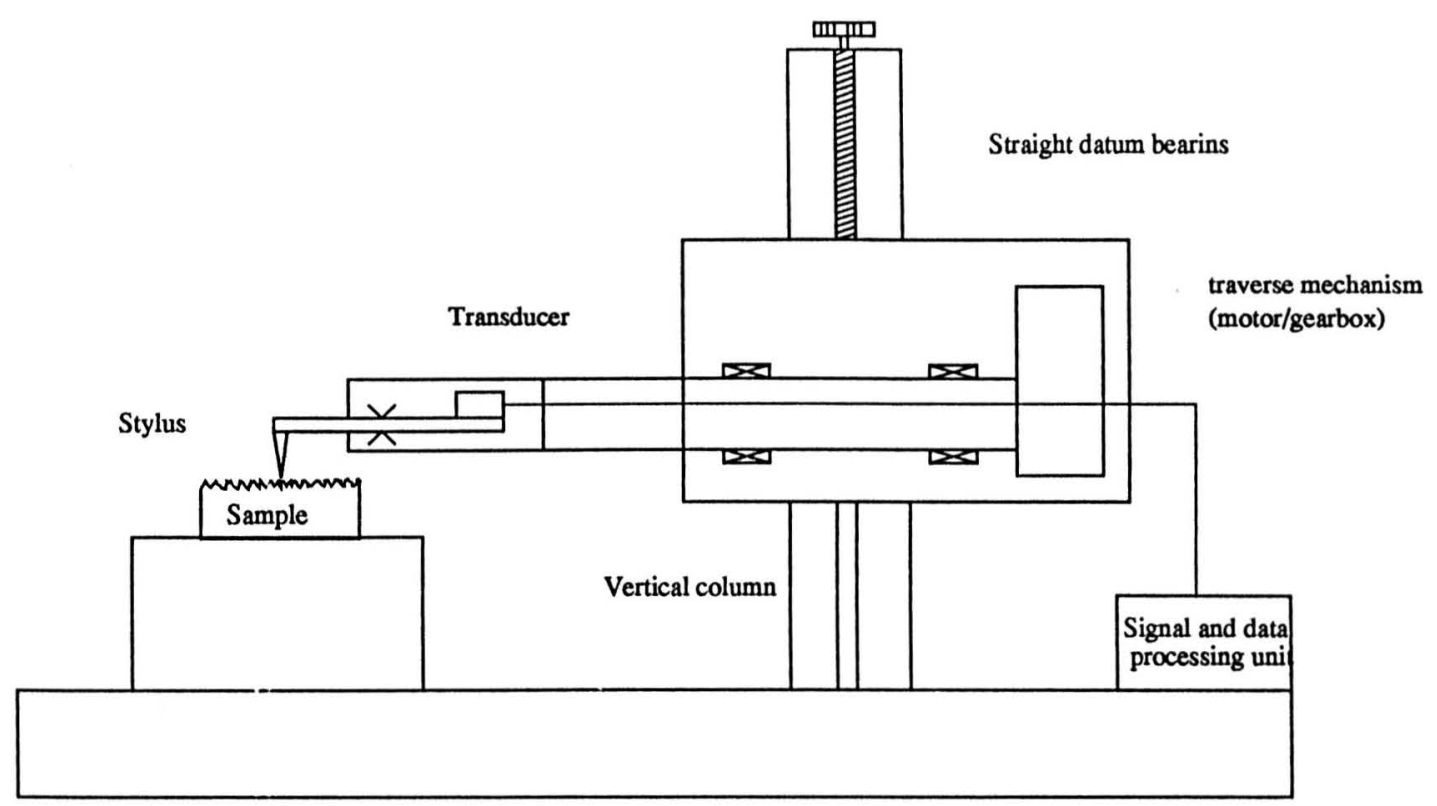

Figure 1.2: Schematic diagram of typical stylus instrument

system uses the measuring surface itself as a reference to generate the datum the locus of the skid center, as shown in Fig 1.3 (a). It functions like a mechanical filter, corresponding to information of small scale surface features, but losing the form and waviness information. Because only local surface "roughness" is of interest, in a majority of engineering applications this type of datum can be widely used. The independent datum is often defined by an optically flat surface on which the stylus traverse arm is rested, as shown in Fig 1.3 (b). This datum system has its mechanical loop open and, therefore, is susceptible to external vibration. Almost all styli are diamond, with the most common shapes being either conical with a $2 \mu \mathrm{m}$ radius tip or $90^{\circ}$ pyramids with the tip truncated to a $2 \mu \mathrm{m}$ square or somelimes $0.1 \times 2.5 \mu \mathrm{m}$ like chisel. The finite dimensions of the stylus inevitably produce a high frequency filter characteristic, exaggerating the radius of peak's curvature and reducing the width of troughs [11] [12]. The interaction between stylus and surface is predominantly a mechanical contact. This makes the measurement less sensitive to surface contaminations and what is measured is truly a mechanical surface. However, the contact forces may produce permanent 


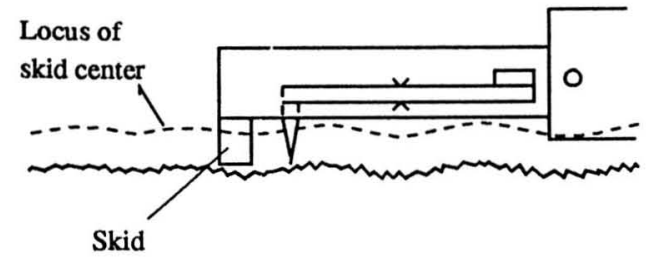

(a) Skid datum

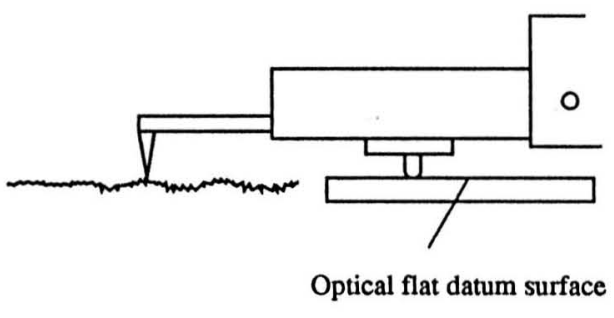

(b) independent datum

Figure 1.3: Schematic diagram of stylus datum systems

deformation and micro-scratches on some engineering surfaces, preventing its use on soft specimens. The relative low dynamic response is another drawback of the instrument, which limits the speed of measurement and makes "in process" measurement difficult.

\subsubsection{Optical methods}

Although the stylus techniques are of great versatility, their drawbacks such as contact deformation and slow response limit their use in many applications. There are many other conventional techniques for surface measurement, such as optical, capacitive and pneumatic methods. Among them, optical methods are considered to be the most obvious complement to the tactile instruments [13]. The optical methods can be generally divided into two categories: focused and area methods.

\section{Focused methods}

In focused methods, a small spot of light beam scans over a surface under measurement. The changes of reflected beam produce the information on the surface texture. There are a large number of focused instruments that can be classified as optical followers, which work on a closed-loop null principle so that the nature 
of the optical change does not have to be fully understood [14][15][16][17]. The feedback signals from photo diode sensors are used to control a servo mechanism which moves objective to follow the surface profile by keeping the light spot in focus. Fig. 1.4 illustrates a simple optical arrangement of the follower deviced by Dupuy based on the principle of the Foucault knife-edge test [14].

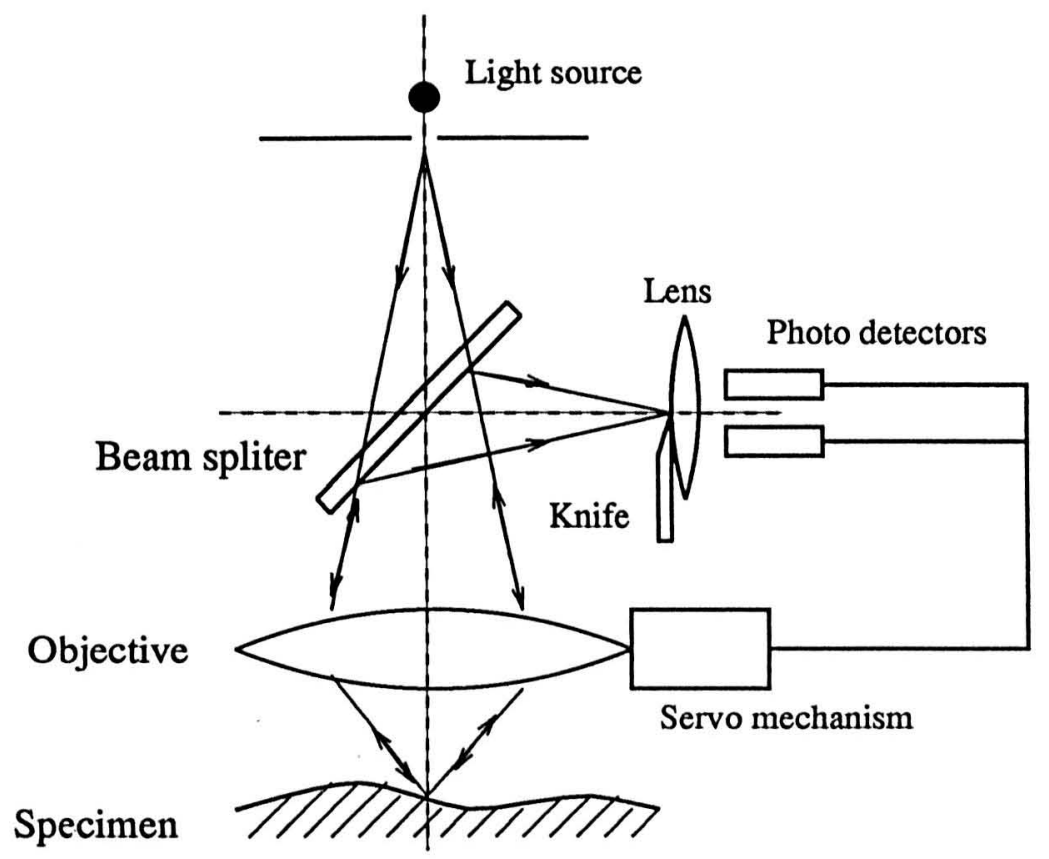

Figure 1.4: Optical arrangement of Foucault knife-edge follower

The defect-of-focus method is a non-follower focused technique [18], which uses a comparison of the differences of the intensities obtained by two photo detectors to sense the relative surface height. Most of these focused methods have a vertical resolution similar to that of stylus technique and the output of these instruments is analogous to the profile traces recorded by stylus instrument. Because of its nature of non-contart and high speed, the instruments based on this or similar principles may become a serious rival to stylus instruments in the market-place as the level of sophistication and accuracy of these devices improves [19]. The fundamental problem associate with the optical stylus to simulate the mechanical stylus is the fact that the focused spot size and the depth of focus are 
limited by the Rayleigh criterion for resolution [1]:

$$
S_{d}=\frac{1.22 \lambda}{\mu \sin \alpha}
$$

where $S_{d}$ is the diffraction-limited spot size, $\mu$ is the refractive index between the medium and the object and $\sin \alpha$ is the effective numerical aperture of the lens. The depth of focus $D_{f}$ is defined as the change in focal position for an increase in beam diameter (if, of $\sqrt{2}$ is given):

$$
D_{f}=\frac{1.22 \lambda}{\mu \sin \alpha \tan \alpha}
$$

However, recent development of scanning near-field optical microscopy breaks the barriers [20] and may make optical technique more competitive in surface metrology (more on these later).

\section{Area methods}

Interference microscopy is a popular technique in area methods. If two slightly inclined glass plates are illuminated by a coherent monochromatic light source, a series of inference fringes will be visible, as shown in Fig. 1.5 (a). The distance between neighbouring fringes is $\lambda / 2$. If these two glass plates are replaced by a reference plane and a specimen, a contour pattern of the surface texture will be generated by the interference between light beams from these two surfaces. There are many types of interferometers such as Newton, Twyman-Green, Fizeau, Michelson and Mach-Zehnder interferometers [21]. Among them, Fizeau interferometer is the most commonly used, the optical arrangement of which is shown in Fig. 1.5 (b). The interferograms can be not only analyzed directly by visual observation, but also digitized using a CCD camera and analyzed by computer with which higher resolution and more detailed information can be obtained [22][23]. In the phase measuring interferometer (PMI), the phase of the cavity is changed in a controlled manner and several interferograms are recorded to allow one of 
many PMI algorithms to interpolate the phase changes in interferogram. Vertical and lateral resolutions of $0.5 \mathrm{~nm}$ and $1.25 \mu \mathrm{m}$ is claimed [23].
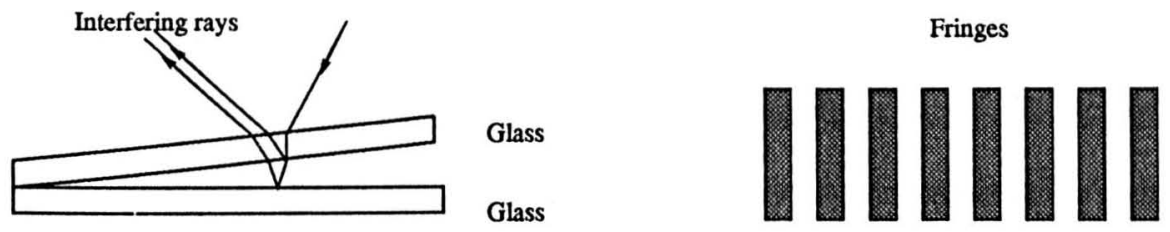

(a)

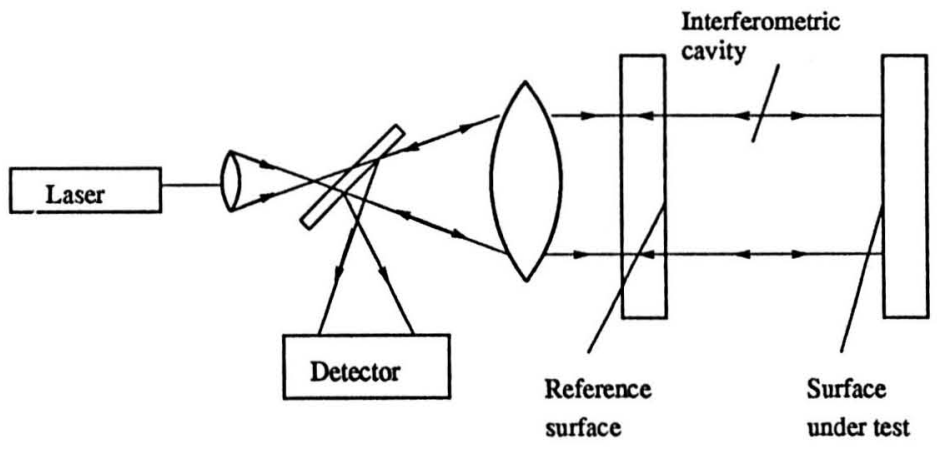

(b)

Figure 1.5: Interferometer for surface metrology. (a) Interference fringes, (b) Fizeau interferometer

\section{Miscellaneous}

All of the optical methods mentioned above measure surface topography directly, while other optical techniques use the reflection of light to quantify surface roughness in parametric terms. Many of these methods can provide surface roughness data in very short time, so they have considerable potential for "in process" application. Scattering measurement is one of these methods, which uses the diffracting properties of the surface to assess its topography [24]. One has to be careful with this method that the theory which reliable predicts the pattern of scattered light is only available for a surface having specific geometric 
properties [25]. The optical scattering instruments give excellent results for the features down to optical wavelengths. To extend the information to subnanometer features, wavelengths of similar order are needed. Recent advances in X-ray instrumentation have made the measurement of surface roughness and topography at nanometer level available by using specular or diffuse X-ray scattering technique [7]. Fig. 1.6 shows a schematic arrangement of X-ray reflectometry. A beam conditioner is used to collimate the $\mathrm{X}$-rays providing a incident beam with very small divergence, and a detector captures the reflected beam from which the surface roughness information is extracted by a computer using an efficient algorithm. The surfaces only show strong specular reflectivity near the critical angle for total external reflection, so that the specimens have to be flat enough to allow the beam to be incident at the grazing angle. The method averages the reflected X-rays over an area of a few square millimeters. The quantitative information on roughness between 0.05 and $5 \mathrm{~nm}$, on correlation lengths from sub-nanometer to tens of micrometers have been claimed [27].

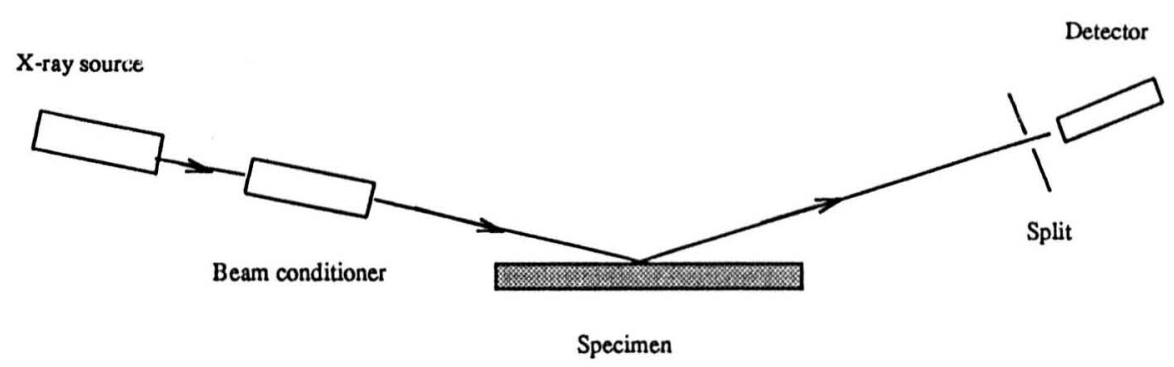

Figure 1.6: Schematic arrangement of X-ray reflectometry

\subsubsection{Modern scanning microscopies}

As the result of rapid development of precision engineering, the requirements on measurement of surface topography have become ever more increased and strict. Many conventional methods based upon stylus and optical techniques can provide a very high vertical resolution, sometimes at sub-nanometer levels. 
However, their lateral resolution is poor, usually of order about one micrometer, which is inadequate for the characterization of current advanced materials and for the application in nano-fabrication technology. Almost all of the modern microscopies such as electron microscopy and scanning probe microscopy have a significant improvement in lateral resolution. Therefore very fine details of surface feature can be distinguished, even sometimes atomic images can be obtained.

Electron microscopy uses electron beams to form magnified images of surface topography. The very short wavelength of the electron beam makes it much more superior to optical methods in respect of lateral resolution and the depth of resolution. A scanning electron microscope (SEM) can resolve to approximately 3 $\mathrm{nm}$ and a transmission electron microscope (TEM) to about $0.2 \mathrm{~nm}$ [28]. The SEM has become a very popular tool for observation and investigation of surface feature in engineering due to its versatility, and the use of TEM is not as popular as SEM because of the difficulty in preparing specimens thin enough to be measured. A basic diagram of an SEM is shown in Fig. 1.7. Electrons from a filament are accelerated by a high voltage and condensed by the condenser lens and then focused by the objective lens. Scanning coils located within the objective lens cause the electron spot scanning over the specimen. A detector captures usually the secondary electrons emitted from the surface and transfer them into signal for display on a CRT screen. The principal drawback of the method is that the interpretation of the images is not necessarily straight forward and they do not readily yield quantitative data about the height of surface feature. Although many attempts have been made to derive such data from the process [29] [30] [31], the results are, so far, still not reliable enough for practical application.

The introduction of scanning tunneling microscope (STM) in 1982 by G. Binnig and $\mathrm{H}$. Roher has had a great impact on surface science. Meanwhile, it has stimulated an entire family of scanning probe microscopes (SPM) which measure a range of physical and chemical properties of a surface on the nanometer or sometimes atomic scale [32]. The SPMs use near-field technique, so that Rayleigh 


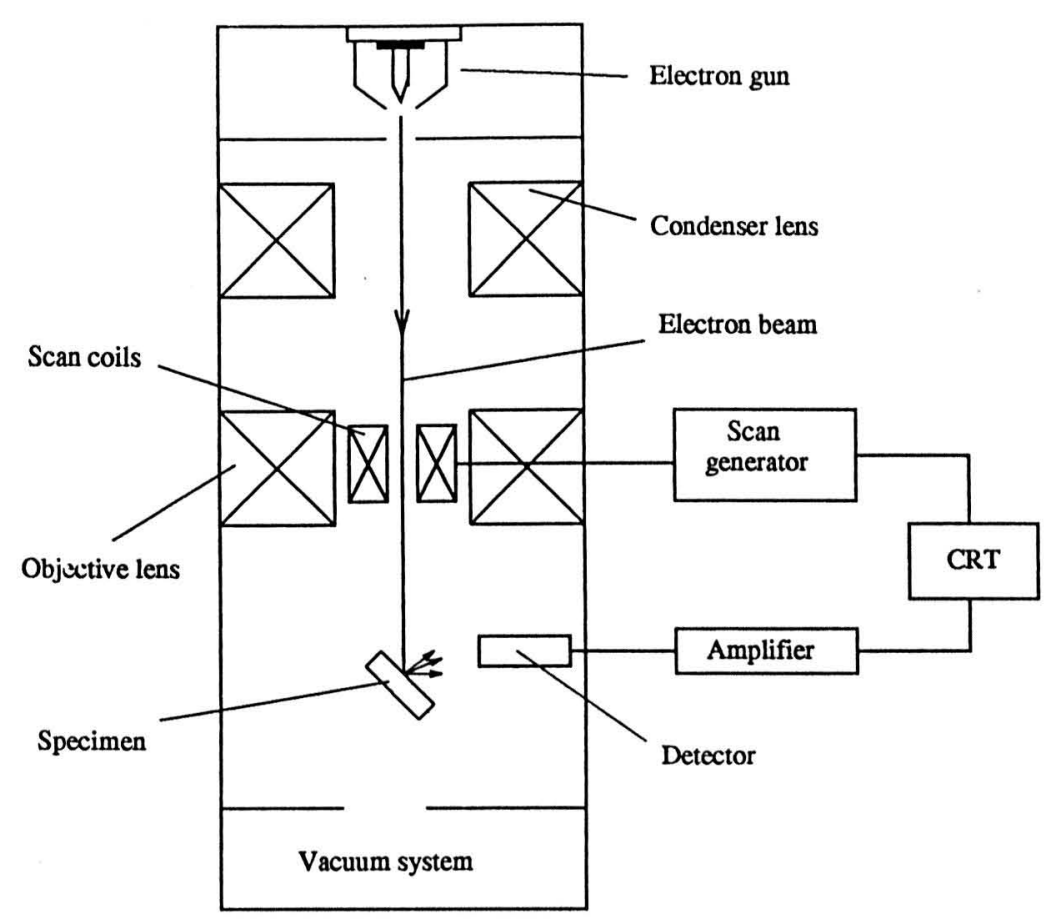

Figure 1.7: Schematic diagram of an SEM

criterion which assumes that the ultimate resolution of optical systems will be limited by the wavelength of the radiation used to form the image is surpassed. The resolution of the SPMs has approached to the physical limit of instrumentation. Their applications have not been limited to surface measurement, but also expanded to surface manipulation for subnanometer machining, single atom manipulation as well as a variety of chemical and biological processes. More details about SPM are reviewed in chapter 2. For surface metrology, scanning tunneling and atomic force microscopes are most commonly used. The atomic force microscope (AFM), or scanning force microscope (SFM), is more versatile for the measurement of surface topography because it can measure not only conducting but also non-conducting specimens. The SFM is similar to stylus techniques in principle, therefore the measurement is that of the mechanical surface. The research work of this thesis is aimed to improve SFM instrumentation with particular emphasis of the metrological function of the instrument, and introduces 
a new SFM instrument suitable for surface metrology. 


\section{Bibliography}

[1] D.J. Whitehouse, 1994, Handbook of surface metrology, IOP publishing Ltd.

[2] I. Sherington and E.H. Smith, 1986, The significance of surface metrology in engineering, Precision Engineering, $179-87$

[3] T.R. Thomas, 1975, Recent advances in the measurement and analysis of surface micro geometry, Wear, $33205-233$

[4] D.J. Whitehouse, 1985, Assessment of surface finish profiles produced by multi-process manufacture, Proc. Instn. Mech. Engrs. 199 Bł, 263 - 270

[5] N. Taniguchi, 1974, On the basic concept of nanotechnology, Proceedings of the ICPE, Tokyo

[6] N. Taniguchi, 1994, The state of the art of nanotechnology for processing of ultraprecision and ultrafine products, Precision Engineering, 16 (1), 5 - 24

[7] D.J. Whitehouse, 1978, Surface metrology instrumentation, J. Phys., E 20 $1145-1155$

[8] D.G. Chetwynd and S.T. Smith, 1991, High precision surface profilometry: from stylus to STM, From instrumentation to nanotechnology, edited by J.W. Gardner and H.T. Hingle, Gordon and Breach Science Publishers, 273 - 300

[9] J.D. Garratt, 1979, Survey of displacement transducers below $50 \mathrm{~mm}, J$. Phys., E 12, 563 - 573 
[10] I. Sherrington and E.H. Smith, 1988, Modern measurement techniques in surface metrology: part I; stylus instruments, electron microscopy and nonoptical comparators, Wear, 125271 - 288

[11] D.J. Whitehouse, Theoretical analysis of stylus integration, Ann. CIRP, 23, $181-182$

[12] V. Radhakirishnan, 1970, Effect of stylus radius on the roughness values measured with tracing stylus instruments, Wear, 16, 325 - 335

[13] R.D. Young, T.V. Vorburger and E.C. Teague, 1980, In process and on line measurement of surface finish, Ann. CIRP, $29435-439$

[14] O. Dupuy, 1967-1968, High precision optical profilometer for the study of micro-geometrical surface defects, Proc. Inst. Mech. Eng., London, 182 (3k), $255-259$

[15] Fang-Sheng Jing, A.W. Hartman and R.J. Hocken, Noncontacting optical probe, Rev. Sci. Instrum., 58 (5), $864-868$

[16] F.T. Arecchi, D. Bertani and S. Ciliberto, A fast versatile optical profilometer, Opt. Comm., 31 (30), 263 - 266

[17] Y. Fairman, E. Lenz and J. Shamir, 1982, Optical profilometer: a new method for high sensitivity and wide dynamic range, Appl. Opt., 21 (7), $3200-3208$

[18] J. Mignot and C. Gorecki, 1983, Measurement of surface roughness comparison between a defected of focus optical technique and the classical stylus technique, Wear, 87, $39-49$

[19] I. Sherrington and E.H. Smith, 1988, Modern measurement techniques in surface metrology: part II; optical instruments, Wear, 125289 - 308 
[20] D.W. Pohl, W. Denk and M. Lanz, 1984, Optical stethoscopy: image recording with resolution $\lambda / 20$, Appl. Phys. Lett., 44, $651-653$

[21] L.A. Selberg, 1994, Interferometric metrology: an introduction, Tutorial of ASPE 9th Annual Meeting, Cincinnati, October

[22] S. So and K. Wong, A hybrid optical-digital image processing method for surface inspection, IBM J. Res. Dev., 27 (4) 276 - 385

[23] J.C. Wyaut, C.L. Koliopoulos, B. Bhushan and D. Basila, 1986, Development of a three dimensional noncontact digital optical profiler, J. Tribol., 108, 1 $-8$

[24] K.J. Stout, 1984, Optical assessment of surface roughness, Precis. Eng., 6 (1), $35-3.9$

[25] J.K. Rakels, 1991, Optical diffraction for surface roughness measurement, From instrumentation to nanotechnoloy, edited by J.W. Gardner and H.T. Hingle, Gordon and Breach Science Publishers, 227 - 254

[26] D.K. Bowen and B.K. Tanner, 1993, Characterization of engineering surfaces by grazing incidence X-ray reflectivity, Nanotechnology, 4, $175-182$

[27] D.K. Bowen and M. Wormington, 1994, Measurement of surface roughnesses and topography at nanometer levels by diffuse X-ray scattering, Ann. of the CIRP $, 43,497-500$

[28] S.L. Flegler, J.W. Heckman, Jr. and K.L. Klomparens, 1993, Scanning and transmission electron microscopy - an introduction, W.H. Freeman and Company, New York

[29] M. Rasigni, G. Rasigni, J.P. Palmori and A. Llebaria, 1981, Validity of surface roughness study using microdensitometer analysis of electron micrographs of surface replicas, J. Opt. Soc. Am., 71 (12), 1549 - 1550 
[30] D.W. Butler, 1973, A stereo electron microscope technique for microtopographic measurements, Micron, 4, $410-424$

[31] Y. Matsuno, H. Yamada and A. Kobayashi, 1975, The microtopography of the grinding wheel surface with SEM, Ann. CIRP, 24 (1), 237 - 242

[32] H.K. Wickramasingle, 1992, Scanned probes old and new, Scanned probe microscopy, edited by H.K. Wickramasingle, American Institute of Physics, New York, 9 - 22 


\section{Chapter 2}

\section{Development of scanning probe microscopes}

Since an atomic image was first achieved by Binnig and his coworkers, 1982 [1] [2], using the scanning tunneling microscope, subsequent developments have resulted in the emergence of an entire family of SPMs from scanning tunneling and atomic force microscopes to those based on sensing techniques of capacitance, magnetic, near-field optic, thermal, ion-conductance and many other near-field physical and chemical properties. From the instrumentation point of view, all these have similar principles of operation with any particular design consisting of

1) A probe transducer system.

2) A three dimension positioning and scanning mechanism.

3) A control system.

4) Data processing and imaging software.

\subsection{Scanning probe microscopy}

\subsubsection{Scanning tunneling microscopy}

The scanning tunneling microscope (STM) is an example of a super resolution microscope capable of atomic imaging ${ }_{17}$ In the STM, the probe consists of a 
fine metal tip which is positioned in close proximity to a conducting surface with a voltage applied between them. The separation between tip and sample is sufficiently small that electrons can tunnel across and therefore generate a current. For an idealized one-dimensional planar tunneling model, when the gap is small and the voltage low, the current-gap distance relation can be simplified to $[3]$

$$
I \propto(V / d) \exp \left(-A \bar{\Phi}^{\frac{1}{2}} d\right)
$$

where $A=1.025(\mathrm{eV})^{-1 / 2} \stackrel{\circ}{A}^{-1}, V$ is the bias voltage between the sample and the tip, $d$ is the gap distance and $\bar{\Phi}$ is average of the barrier height between the two electrodes. It indicates that the tunneling current varies exponentially as gap distance changes. Actually, $1 \stackrel{\circ}{A}$ change in the gap distance can produce an order of magnitude change of the tunneling current with $\bar{\Phi} \sim 4 \mathrm{eV}$. By this, displacement of $10^{-4} \stackrel{\circ}{A}$ can be measured. This phenomenon was first introduced into metrological application as a field emission ultramicrometer by Young, 1966 [4], and possible applications of contact free measurement of surface profiles or surface contours were proposed. After that, the first surface profile measuring instrument based on field emission was invented by Young et al. 1972 [5], called "Topografiner". In this instrument, a tungsten tip (emitter) is held on an $x-y-z$ piezoelectric translation stage. The specimen is brought to the probe close enough so that it is within the range of $z$ piezo translator using a differential micrometer. The current in the gap is fed back to a servo controller to maintain a constant field emission current and hence a fixed gap distance. As the tip is scanned laterally across the specimen, the changes of $z$ piezo driver voltage are interpreted into the variations of surface height. Although the subnanometer resolution was not achieved by this instrument, tunnelling was demonstrated and STM instrumentation projosed. Ten years later, a working scanning tunneling microscope system was built by Binnig and Rohrer, 1982, [1] [2]. Their device had good vibration isolation and stable feedback servo controller and was maintained under UHV condition. The tip is kept close to the sample with even smaller gap sepa- 
ration of a few Angstroms, so that a tunneling current is obtained. At this time, atomically resolved images were obtained such as topographic maps of $C_{a} I_{r} S_{n 4}$ and $A_{u}$ (110) surface and an image of the $7 \times 7$ Reconstruction on $S_{i}(111)$ [6] . Stimulated by these results, there was a boom in research to further understand tunneling mechanisms, improve instrumentation and explore new applications of this technology. Therefore, their work was generally considered as a pivot for the development of whole range of scanning microscopes based on local probes.

\subsubsection{Scanning force microscopy}

Although the STM conducts atomical imaging successfully, it operates only with conducting surfaces. This prevents its normal application on an insulator or in a working environment in the presence of surface contamination. The invention of the atomic force microscope (AFM), also by Binnig and his coworkers 1986 [7] solved this problem. The AFM probe measures minute changes in force between the tip and the surface as a means of proximity detection. Therefore, it can image nonconducting as well as conducting sample surfaces.

In the AFM, a sharp tip is attached to the end of a small cantilever beam which bends due to van der Waals interatomic (or intermolecular) forces as the tip is brought into near proximity to the surface. The deflections of the beam are monitored with a sensitive detector. The signals from the detector are used to servo a vertical piezoelectric translator on which the force probe (or specimen) is mounted to maintain a constant force acting between the tip and the specimen as the tip scans across the surface, as shown in Fig. 2.1. The short range nature of the interatomic, or intermolecular, forces makes it possible for the AFM to get high resolution in both the vertical and lateral directions. Atomic resolution images have also been achieved using this technique [8].

Interatomic forces acting between any two solids can be divided into repulsive or attractive, short or long range. It is found experimentally that in the absence of contaminants and in equilibrium, typical interatomic spacings are on the order 


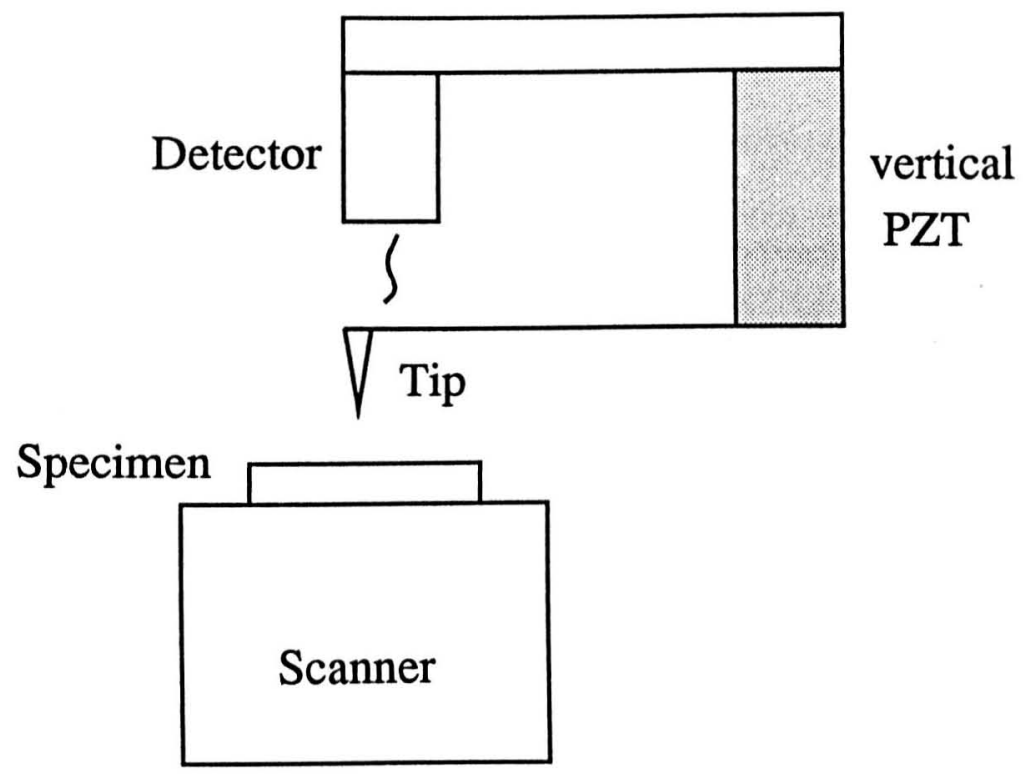

Figure 2.1: Schametic diagram of the configuration of atomic scanning microscope

of 2 to $3 \stackrel{\circ}{A}$ [9], below which interatomic forces are always repulsive, and above which they appear attractive within a certain range. According to Lennard-Jones potential, the main forces involved in the interatomic interactions can be classified as

A) electrostatic or Coulomb interactions between charges or charge distribution, such as mınopoles, dipoles, quadrupoles, and their combinations.

B) polarization forces, where a distribution of charges in one molecule create a dipole moment in an adjacent molecule.

C) quantum mechanical forces, which give rise to covalent bonding and to repulsive exchange interactions.

The experimentally derived Lennard-Jones molecular interaction energy, combining the attractive van der Waals and repulsive atomic potentials, provides an easily understood view of general properties of these interaction forces:

$$
w(r)=4 w_{o}\left([\sigma / r]^{12}-[\sigma / r]^{6}\right)
$$


The forces are obtained by differentiating equation 2.2 , yielding

$$
F(r)=24 w_{o}\left[\sigma^{6} / r^{7}-2 \sigma^{12} / r^{13}\right]
$$

where, $r$ is distance between two interacting atom systems, $w_{o}$ and $\sigma$ are experimentally derived constants. This force and distance relationship is plotted in Fig. 2.2 without a scaling for demonstration purpose,

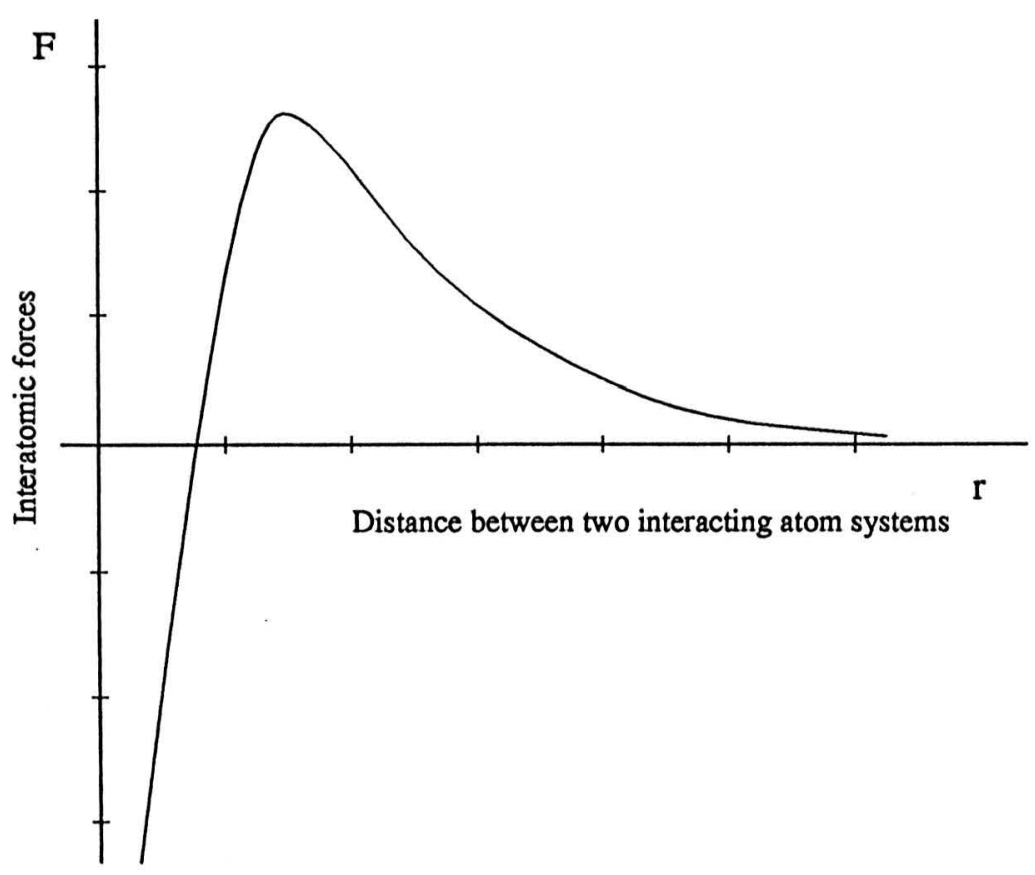

Figure 2.2: The plot of interatomic force

The real cases for these interatomic forces are far more complicated and are not encountered in AFM which 'see' only many body interactions. Discussions on that are beyond the scope of our interests in the thesis. More details can be found in references [9][10].

Corresponding to the characteristics of the interatomic forces, there are two distinct operation modes of the AFMs, attractive (noncontact) mode or repulsive (contact) mode. In the first, the probe is approached to the surface until a predefined attractive force is sensed. By servo controlling to maintain this force 
while the surface is moved relatively to it, the probe will move following the contour. The second mode is achieved by reducing the probe to surface separation until a repulsive force is encountered. This is directly analogous to conventional stylus profilometry only the force is maintained at a constant value which may be in the region of nanonewtons, which is three to six orders of magnitude lower than the forces commonly used with the stylus. Because of the short range nature, the AFMs working in repulsive mode are capable of achieving atomic resolution. In contrast, attractive mode AFMs operate with relatively large tipsample distances and it is therefore difficult to reach the same resolution. Its probe usually has interacting forces which are much smaller than that encountered by repulsive mode AFM, so that resonance enhancement techniques are required. In this case, the cantilever beam is vibrated and the changes of the vibration caused by small variation of the attractive forces or force deviatives are monitored and interpreted into profile information. Sarid gave a good summary on the enhancement $t \in$ chniques in his book [9].

Attractive mode scanning force microscopes have been used to conduct a number of novel measurements ranging from non-contact profiling of surfaces to magnetic [12] and electrostatic imaging [13].

In magnetic force microscopy (MFM), the tip is replaced by a magnetic one typically iron or nickel and a magnetic interacting force between tip and sample is detected. The topography measured over a flat magnetic surface can be directly related to its magnetic features.

In the electrostatic force microscopy (EFM), an AC voltage is applied between tip and sample and the induced force is sensed. The force is proportional to the square of the applied voltage $V^{2}$ times the rate of change of tip-sample capacitance with spacing, $\partial C / \partial r$. By keeping the spacing constant the surface topograph can thus be measured. 


\subsubsection{Scanning near-field optical microscopy}

In a long history of the development of optical microscopy, it was conventionally believed that the ultimate spatial resolution that can be achieved in any optical system is roughly one half the wavelength of the radiation used to form the images - the so called Rayleigh criterion (from equation 1.1). This limit was not surpassed until the invention of scanning near-field optical microscopy (SNOM). In this technique, an optical probe with a tiny aperture at the end of the tip is illuminated from the base side and scanned along the sample in very close proximity, typically less than $5 n m$. As a result, a scanning optical micrograph of the surface can be obtained, in which the lateral resolution is determined by the tip probe diameter and not by the wavelength of the radiation. Since the optical probe tip can be fabricated to the order of $10 \mathrm{~nm}$ in diameter, SNOM offers the possibility of spatial resolution far exceeding the Raleigh limit. The papers written by Durig et al. [14] and Isaacson et al. [15] give a good introduction and review of this technique.

\subsubsection{Scanning capacitance microscopy}

Capacitance has been used as the basis of a local probe resulting in the development of scanning capacitance microscopy (SCM), which provides a means for surface characterization through the measurement of local capacitance between tip and sample. A conductive tip is usually used as one of the electrodes and the other electrode, sample, can be either conductive or nonconductive. The capacitance or local charge storage between the proximal tip and sample forms the interaction. Therefore, both conductors and insulators can be profiled with SCM. If feed back control is used to scan the tip at constant gap across a sample, noncontact surface profiling can be realized. Explored by Matey [16], the scanning capacitance microscope demonstrated a vertical resolution of $0.3 \mathrm{~nm}$ and a lateral resolution of $0.1 \mu \mathrm{m}$ by $2.5 \mu \mathrm{m}$. The system of Bugg and King [17] has been developed into a commercial instrument with lateral resolution of $2 \mu m$. Using 
a near-field capacitance microscope built by Williams et al. [18], a capacitance image with resolution less than $25 \mathrm{~nm}$ has been achieved. Another unique capability of the technique is that it can measure buried conducting layers. This is potentially useful for semiconductor applications.

The capacitance method suffers from the problem that it measures variation in dielectric as well as topography. With current microscope designs the sensitivity is not as high as other near-field probe techniques.

\subsubsection{Scanning thermal microscopy}

Thermal imaging is another novel application of SPM. Scanning thermal microscopy (SThM) is to measure thermal interactions between tip and sample using SPM technique. The first scanning thermal microscope was built by Williams and Wickramsinghe (1986) [19], which aimed to overcome the inability of the STM to image non-conductive surfaces since the AFM was not well developed at that time. The thermal probe consists of a thermo-couple junction built at the end of a tungsten tip ( $50 \mathrm{~nm}$ in diameter). The probe is heated and brought within near proximity of surface. The thermo-couple junction is used to measure the temperature change due to the conduction of heat between the tip and sample via air. This provides the feedback signal to maintain constant tip-sample spacing during lateral scan. Because of the large difference in thermal conductivities between air and solids, the measurements tends to be independent of the material properties. However, temperature changes on the scan surface may appear as features on surface image thus making it difficult to separate temperature from topographical variations, and the surface temperature can not be imaged separately. These limit the application of the technique.

Weaver et al. [20] modified a STM to make a tunneling thermometer in order to measure optical absorption of thin metal film, achieving a $1 \mathrm{~nm}$ spatial resolution. More recently, atomic force microscopes have been used for thermal mapping by integrating a thermo-couple into the force probe [21][22][23]. Using 
this method, simultaneous thermal and topographical measurement becomes possible and thermal image and topographical image can be obtained separately. So called "passive" and "active" thermal measurement modes can be implemented. In the "passive" mode, the thermo-couple is only used to measure the surface temperature, so that temperature image is obtained. In the "active" mode, the thermo-couple is heated. The heat flow between the probe and sample will be influenced by the thermal conductivity of the sample and the temperature difference. In this case, it is possible to image the thermal properties of the sample surface or subsurface.

\subsection{SFM transduction methods}

A force detection system is essential for the realization of the scanning force microscopies mentioned above. Common to the force detection system is the use of a delicate transducer that measures the minute force-induced deflections of a flexible cantilever supporting the force sensing tip. The output of this is used to maintain the constant force feedback. Many possible sensing techniques have been tried for the deflection monitoring, of which some of the more successful are outlined below.

\subsubsection{Tunneling detection system}

In the first atomic force microscope invented by Binnig et al. [7], electron tunneling was used for its force detection system. The working principle of the tunneling detection system is shown schematically in Fig. 2.3. In this system, the minute deflections of the force sensing lever is monitored by a STM probe which measures the tunneling current from the back of the cantilever. The signals from the STM probe are fed to the servo controller for the $z$ translator of specimen to maintain constant contact force between the tip and the specimen. By modulating the tunneling current it is possible to measure displacements as 


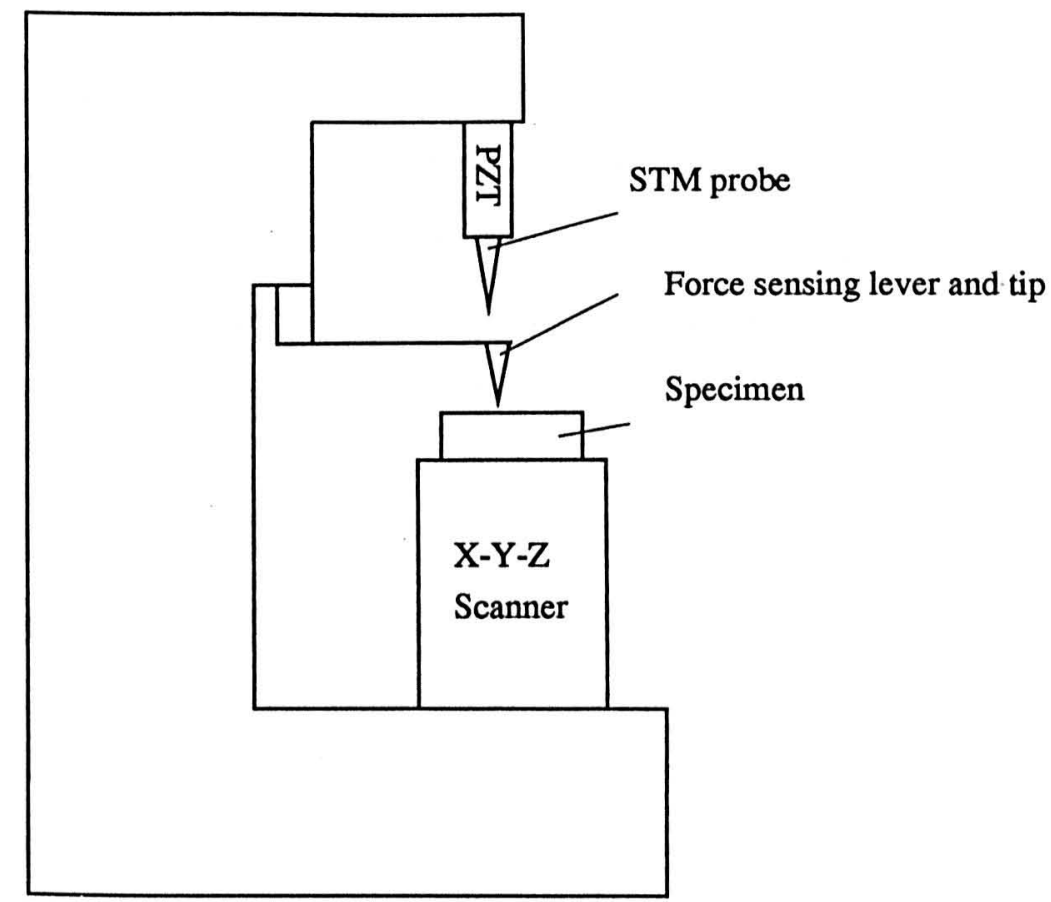

Figure 2.3: Schematic diagram of tunneling detection system

small as $10^{-4} \stackrel{\mathrm{C}}{.}$, corresponding to a force of $10^{-16} \mathrm{~N}$ if a cantilever of $0.01 \mathrm{~N} / \mathrm{m}$ in stiffness is used, and by vibrating the lever, the sensitivity to force can be further increased [7]. Atomic resolution has been achieved using the AFM with tunneling detection system by Binnig and Rohrer 1987 [25], Marti et al 1987 [26] and Kirk er al. 1988 [27]. However, some problems were encountered in its applications. Local topography of the cantilever surface and contaminants on tunneling interfaces can be detrimental to the stability of the tunneling current. The proximity of the tunneling tip and the cantilever will also introduce the effect of interaction atomic forces, resulting in further interpretation problems of measurement results. These problems make it unreliable for use as the tip force detector in air or even in UHV. Although it is an extremely sensitive transducer for high resolution displacement, it has become less favoured for force detection because of its local nature effects mentioned above. 


\subsubsection{Capacitance detection system}

Capacitance displacement detection is an alternative method for monitoring the minute deflections of the force sensing cantilever. Fig. 2.4 shows a diagram of capacitance based force probe system. A stationary electrode is located very close to the back face of the force sensing cantilever to form a capacitance sensor. The gap between the electrodes is usually in the region of a few micrometers. The capacitance changes caused by force-induced cantilever displacement can be detected using a transformer bridge. Again, the signal from the capacitance gauge is used for servo control of $z$ PZT to maintain constant contact force during scanning. The capacitance detection measures the average effect of the two electrode surfaces, so that it is much less susceptible to the local conditions of detecting area than STM detection. Therefore it is a more stable and reliable detection method and can be made compact in structure. In previous work by T. Goddenhenrich, et al. using capacitance detection in their magnetic force microscope, a minimum force $10^{-10} \mathrm{~N}$ was reported [28]. The SFM built by G. Neubauer, et al., [29] using bidirectional capacitance sensing technique for simultaneous measurements of normal and lateral forces, achieved a vertical resolution of $10 \AA$ and noise level of $0.03 \AA$ RMS. Design details of the capacitance based force probe will be discussed in Chapter 3.

Another novel application of the capacitance sensor is its additional use for electrostatic force balance. Generally, the force sensing modes of AFM can be divided into two classes simply known as " $d c$ " and "ac" modes. In the $d c$ microscope, the interaction force between tip and sample is directly sensed through the static deflection of a weak cantilever when the tip is in the proximity of the sample. To achieve high sensitivity requires a low spring constant. It, however, makes operation in the attractive mode difficult. When the probe-sample force gradient, $\partial F / \partial z$, where $z$ is the surface separation, exceeds the spring constant of the cantilever, instability of cantilever-tip-sample potential will occur, i.e. the tip is pulled into the surface. " $a c$ " microscopes can overcome this problem by detect- 


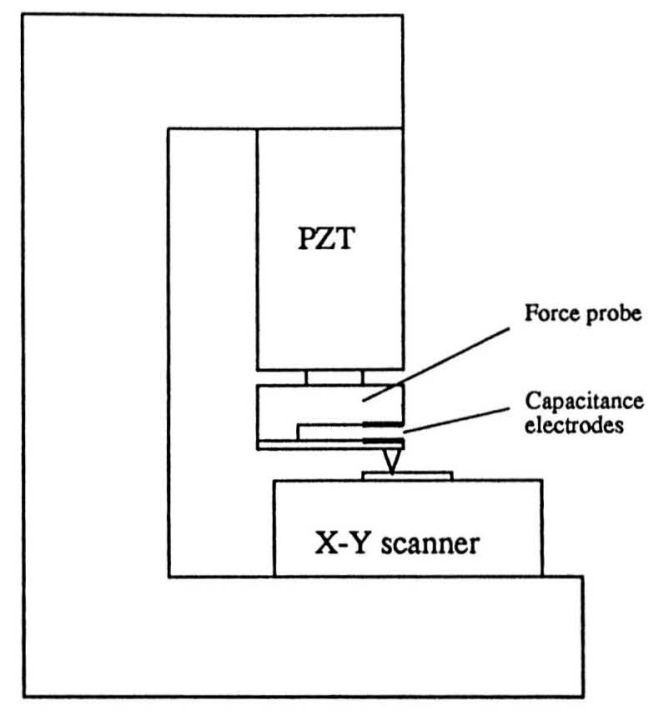

Figure 2.4: Schematic diagram of capacitance detection system

ing the force gradient through variations in either the amplitude or the frequency of a vibrating stiffer cantilever. In this way, however, the force is not directly available. By using the cantilever having large spring constant in $d c$ mode, the instability can be reduced, but the force sensitivity will decrease as well. A solution for the problem is electrostatic force balanced force probe explored by Joyce and Houston (1991) [30] and Miller et al. (1991) [31]. Fig. 2.5 shows a schematic diagram of the probe. A force sensing beam, with a tip mounted on the end of it, is centrally surported by a torsion bar which is, in turn, rigidly fixed on a solid base. A small gap exists between the beam and the base. Two electrodes (C1 and $\mathrm{C} 2$ ) on the opposite surfaces of them forms a differential capacitance sensor monitoring bearn's position using an rf phase shift technique.

A positive voltage is applied to each of the two electrodes inducing an electrostatic force $r_{c}$ on the beam, which is given by

$$
F_{c}=\frac{\varepsilon A V^{2}}{2 d^{2}}
$$

where $d$ is the gap between electrodes and $A$ is the over covered area of the 

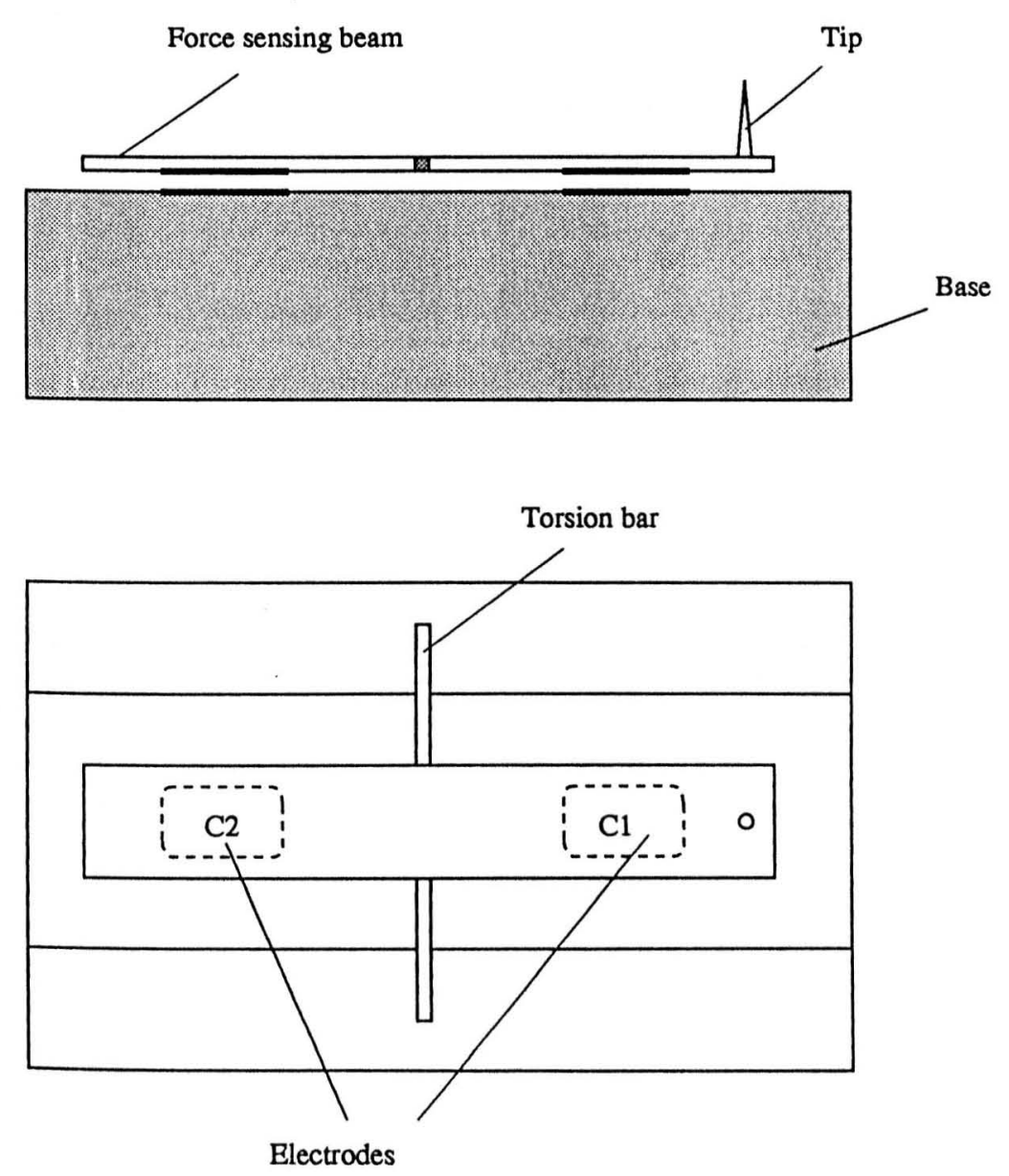

Figure 2.5: Schematic diagram of electrostatic force balanced force probe 
electrodes. When the tip approaches the sample the interaction force between tip and sample will cause the beam to rotate about the torsion bar, resulting in the gap change of each capacitance sensor, increasing one and decreasing the other. The signal from the sensor is fed back to a servo control system which applies a restoring force, by applying a positive voltage, to the appropriate electrodes. The gap change can be counter-balanced, i.e. the interaction force is counterbalanced, maintaining the beam at its rest position. This force feedback control, therefore, eliminates the instability or jumping problem and enables a higher sensitivity and an electrostatic stiffness.
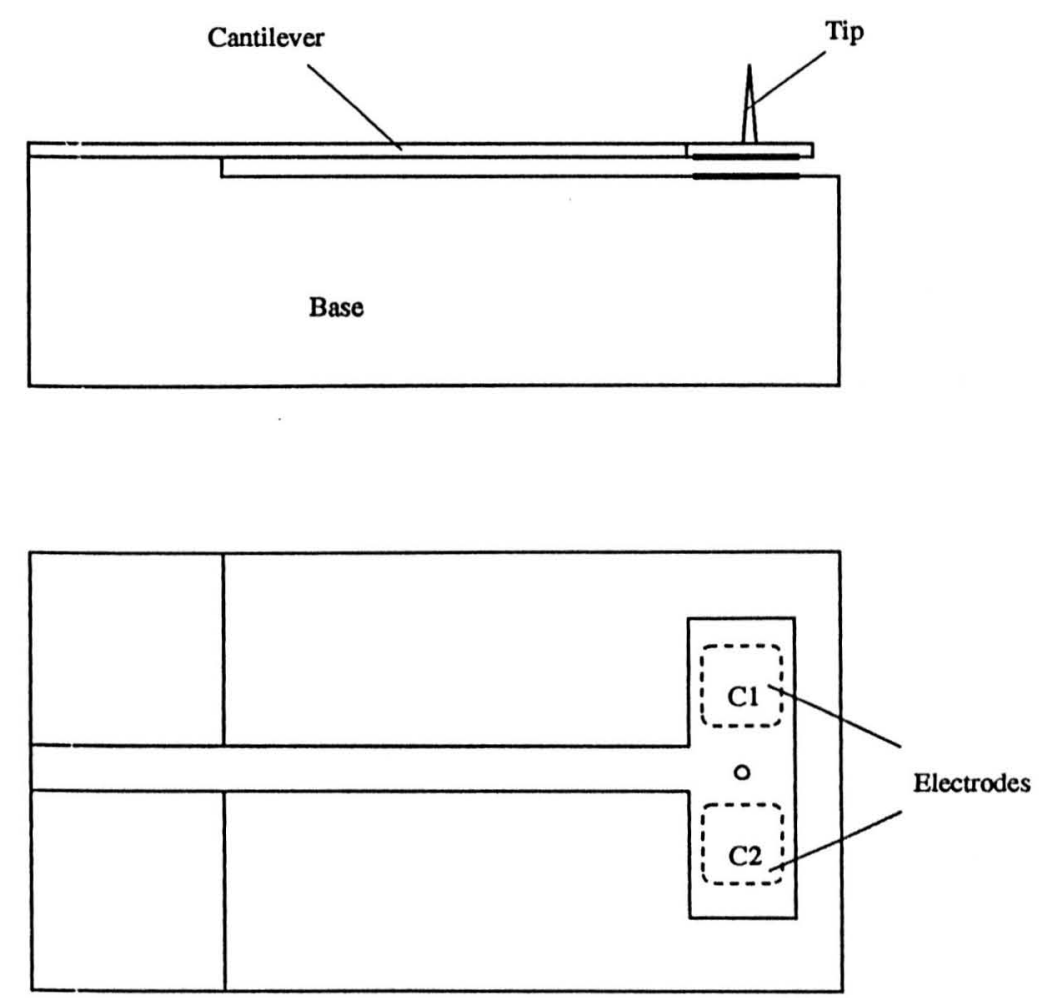

Figure 2.6: Schematic diagram of a high stiff tribological force probe

As an exter.sion of this technique, a high stiffness tribological force probe is in investigation in our laboratory at the University of Warwick [11]. In this probe, schematically shown in Fig. 2.6, a tip is mounted on a $T$ shape cantilever and the bottom end of the cantilever is fixed on the plateau of a solid base. 
Two electrodes at the top end of cantilever form a differential capacitance sensor monitoring the normal bending as well as twist of the cantilever. Correspondingly, both normal forces and lateral forces can be measured. By applying a voltage to each of these electrodes through servo control the twist of the cantilever can be counter-balance $\mathrm{d}$ by induced electrostatic forces. The stiffness of the probe can be increased in both normal bending and twist without reducing the sensitivity.

\subsubsection{Optical detection system}

Application of various optical techniques has become more and more popular in development of force detection system of SFMs. Some advantages of optical detection over tunneling detection of the lever deflection are quite clear. It is more reliable and easier to implement, it is insensitive to the roughness of the lever, and it has a lower sensitivity to thermal drifts [12]. These techniques include optical beam deflection, laser diode feedback detection and interferometer detection. All these methods are very sensitive for measuring the deflection of force sensing cantilever and, ideally, atomic resolution can be achieved.

\subsubsection{Optical beam deflection}

Optical beam detection technique was firstly used in scanning force microscopy by Meyer and Amer (1988) [32] [33]. The working principle of the detection system is shown in Fig. 2.7. A collimated laser beam is focused on a reflective surface on the back of force sensing cantilever, and is reflected back into a position sensitive detector. The force induced deflection of the cantilever will produce a relative angle change $\delta \alpha$ between the reflecting surface and incident light beam, resulting in a $2 \delta \alpha$ angle change of reflected light beam. The detector can be as simple as a split photodiode, whose photocurrents are fed into a differential amplifier. Two segmented photodiode can be used for single beam deflection monitoring. 
The angle change of the reflected light beam causes changes in received light distribution on the sensing elements resulting in one segment collecting more light than the other, the output of differential amplifier is used for imaging force across a sample. If four segmented photodiode is used, normal deflection and twist of the cantilever can be measured with one laser beam, i.e. the normal forces as well as lateral forces can be monitored simultaneously.

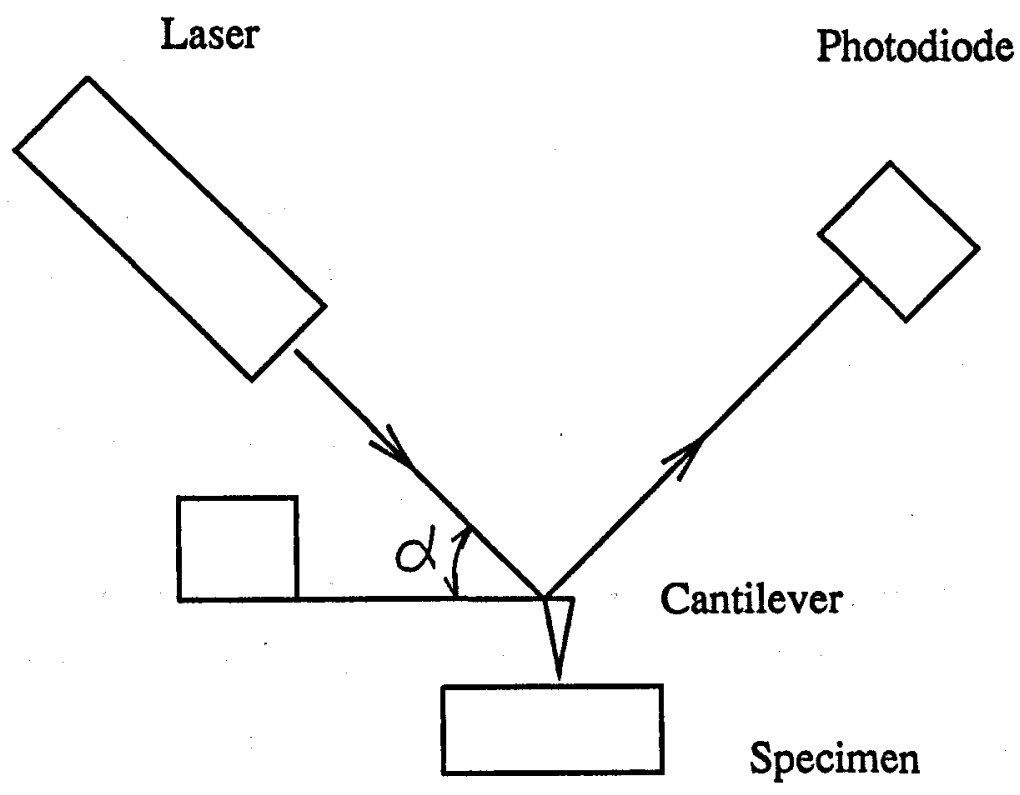

Figure 2.7: Schematic diagram of optical beam deflection detection system

Both theoretical analysis [34] and experiment results [35] [36] indicate that this method has similar sensitivity as the more complex interferometers. More over, it is simple in structure and reliable in performance so that it has been widely used in laboratory and commercially available AFMs. Atomic corrugations on a $\mathrm{NaCl}$ (001) surface were imaged in ultrahigh vacuum by Meyer and Amer [35] using an optical-beam-deflection atomic force microscope operating in the short-range repulsive regime. Alexander et al. [36] presented a atomic-resolution image of "native oxide" silicon surface, using similar optical lever AFM. The lateral forces between tip and surface were measured by Taubenblatt [37] by combining the optical lever technique into a modified AFM. Developed at the University of 
Utah by Wenzler et al.[38], an integrated scanning tunneling, atomic force and lateral force microscope has achieved simultaneous measurement of normal and lateral forces successfully using this optical technique.

\subsubsection{Laser-diode feedback detection}

Laser-diode feedback detection is a quite new technique compared with the other two optical methods used in atomic force microscopy. It takes advantage of the extreme sensitivity of a laser diode to optical feedback to obtain the same sensitivity as that of other optical techniques.

In a laser diode detection system, shown schematically in Fig. 2.8, a force sensing cantilever is mounted close to a laser diode, about several micrometers from the front facet of the laser. The reflective surface of the cantilever and front facet combine to act as a Fabry-Perot etalon. A minute deflection of the cantilever modifies the reflectivity of this effective front mirror of the laser diode strongly affecting the threshold current of the laser diode. A photodiode is integrated into the laser diode package, located close to the back facet of the laser diode, monitoring the light emitted from it. The signals from the photodiode can then be used for AFM imaging. No other optical components, such as mirrors, lenses and fibers are necessary in this system. It is therefore simple to assemble and easy to align. Developed at the University of Arizona by Sarid et al. [39] [40] [41], the atomic force microscope using a laser diode detection system of a sensitivity of 3 $p m$ per $\sqrt{H_{z}}$ has been used in the noncontact mode to image magnetic domains and in the contact mode to image large area samples. The improved version of the AFM uses commercially available mechanical and electronic components of the laser diode package leading to an atomic resolution [42].

\subsubsection{Interferometer detection}

Interferometry is a time-tested and well understood method for precision measurement of displacement. Various interferometric techniques have been applied 


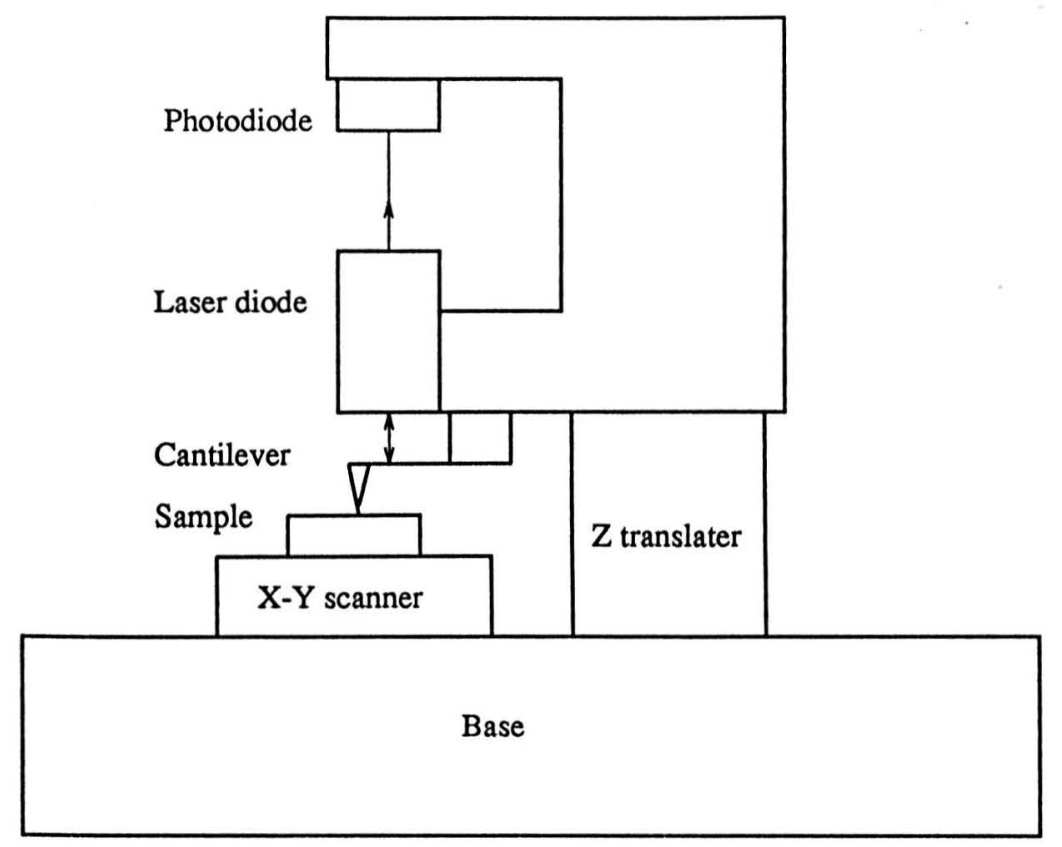

Figure 2.8: Schematic diagram of laser-diode feedback detection system

to atomic force microscopy for force sensing detection.

McClelland et al. (1987) [43] and Erlandsson et al. (1988) [44] employed a homodyne interferometer in atomic force microscopy. In this detection system, as shown schematically in Fig. 2.9, a polarized beam from a HeNe laser is incident on a polarizing beim splitter and goes through a quarter-wave plate. The quarterwave plate togeiher with the polarizing beam splitter serve as an optical isolator to prevent stability problems due to retro reflection of the laser beam. The beam continues towards a flat half mirror and part of it is reflected back passing again through the quarter-wave plate and deflected by the polarizing beam splitter into a photodiode. The second part of the beam passes through the flat half mirror and is focused onto the force sensing cantilever and is reflected back. The beam then traverses the same path as the first beam back to the same diode. The interference between these two beams is affected by the optical path length difference between the half mirror and the cantilever.

This interferometer has an excellent sensitivity when used for $a c$ displacement 


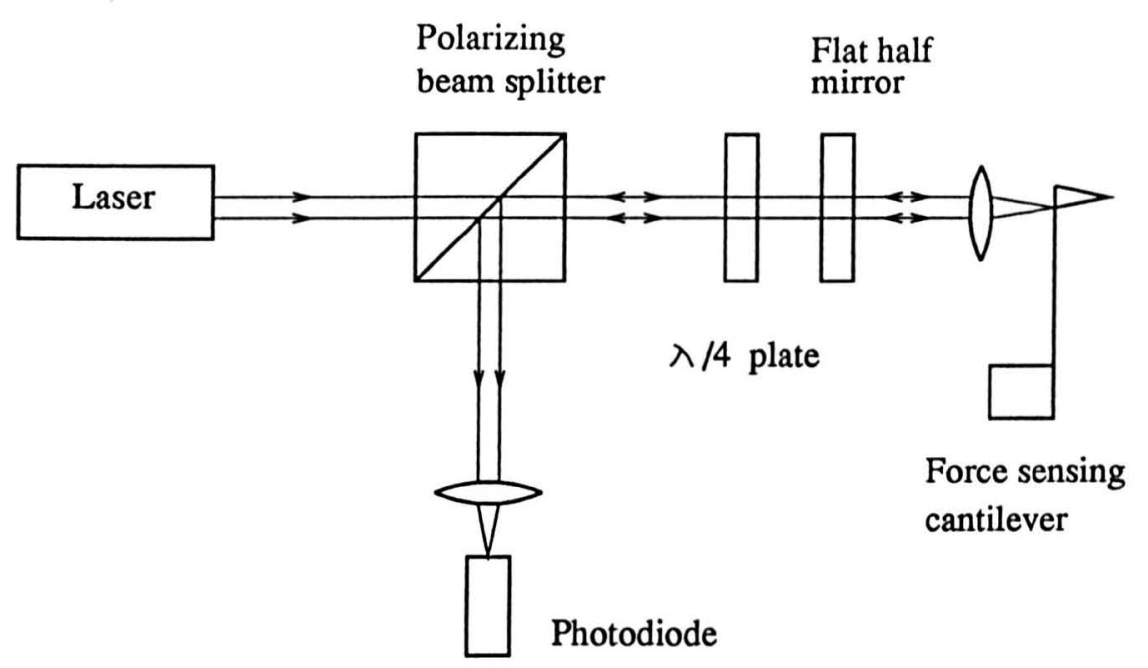

Figure 2.9: Schematic diagram of homodyne detection system

measurements. However, since this system provides no particular immunity to optical path length fluctuations it is not well suited to low-frequency or static measurements. In recognition of this deficiency, Rugar et al. [45] improved the Michelson interferometer used by Erlandsson et al.. By guiding the light in an optical fiber they were able to minimize the interferometer cavity and thereby improve the $d c$ stability. Later, it was improved into a differential fiber-coupled homodyne detection system, shown schematically in Fig. 2.10 [46] [47] [48]. The light from a laser diode is coupled into the input of a $2 \times 2$ single-mode directional coupler through a single mode optical fiber. The coupler splits the light power equally into two parts. One part is carried by a fiber to the reference photodiode and the other part is guided by the fiber which is placed up to a few micro meters from the reflective surface of force sensing cantilever. Approximately $4 \%$ of the light in the fiber is reflected at the cleaved end of the fiber. The remainder exits the fiber and is partially scattered from the cantilever back into the same fiber. There, it interferes with the light reflected from the fiber end and is directed by directional coupler into the fiber leading to the signal photodiode. The outputs of the two photodiodes are compared electronically and the difference between these 
two is used for force imaging. In this way, the laser amplitude noise is cancelled and better performance is obtained, especially at frequency below $1 k H_{z}$ with peak to peak noise less than $0.1 \stackrel{\circ}{A}$.

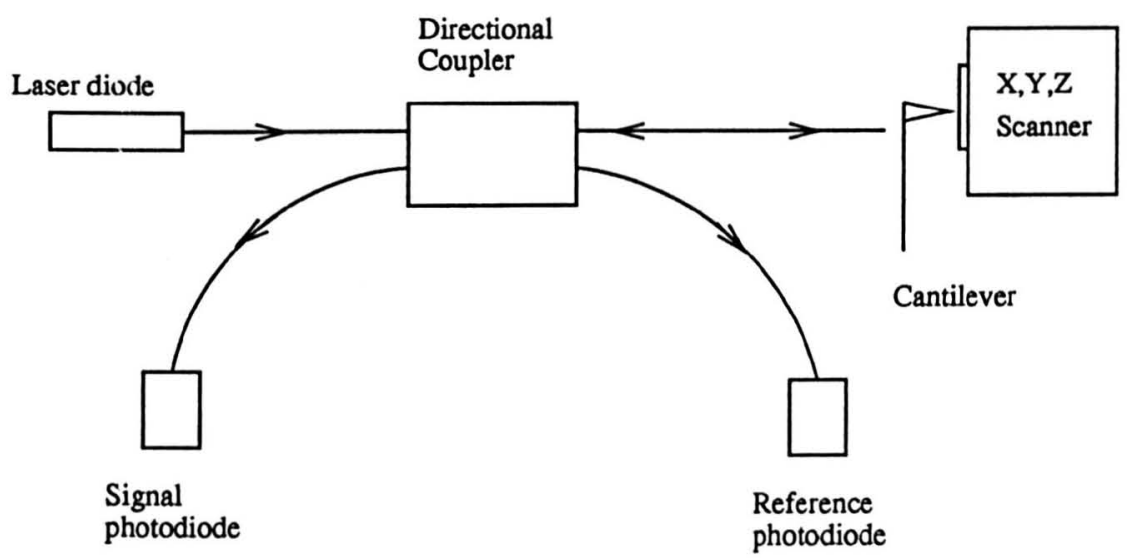

Figure 2.10: Sichematic diagram of fiber-optic interferometer detection system

Various differential interferometers have been developed and employed in atomic force microscopy. In the optical detection systems build by den Boef (1989) [49], and Schonenberger and Alvarad (1989) [50], the reference beam and signal beam are orthogonally polarized. A very low noise level of $0.01 \AA$ (rms) has been achieved in a frequency range from $1 k H_{z}$ to $20 k H_{z}$ [50].

Laser heterodyne interferometer was also employed in atomic force microscopy by Martin and Wickramasinghe (1987) [51]. Using this detection system, static and dynamic magnetic force fields and electric force fields were measured [51][52] [53][54]. The unique feature of the heterodyne detection system is that it is independent of optical path length drifts so that the stability can be improved.

\subsubsection{Others}

Besides the force detecting techniques introduced above, people are putting efforts in exploring more simple and compact force sensing methods for AFMs. Some sensors are fabricated directly onto force sensing cantilevers, and others 
use cantilever material itself to form a sensor. Itoh and Suga at University of Tokyo reported a force probe for non contact AFM, in which a piezoelectric film with electrode on each side was deposited on a cantilever. The deflection of the cantilever can be monitored by detecting the induced charge between the electrodes when using piezoelectric vibrator, or the current through the piezoelectric layer when cantilever is vibrated by a voltage between the electrodes. The smallest detectable derivative of the force and the minimum detectable change rate of the lever amplitude (amplitude change / amplitude in free vibration) were claimed to be $1.8 \times 10^{-3} \mathrm{Nm}^{-1}$ and 0.017 , respectively [55].

Using the piezoresistive effect of p-type silicon, Park Instruments developed a force probe using cantilever material itself as a strain gauge. The deflections are measured by connecting the force probe as one element of a Wheatstone Bridge. The minimum detectable deflections of around $0.2 \mathrm{~nm}$ with a bandwidth of 1 $k H_{z}$ was reported [56]

\subsection{Scanning and coarse positioning mechanism}

\subsubsection{Scanning mechanism}

Imaging or profiling surfaces to high resolution in both vertical and lateral directions is a unique capability of scanning probe microscopy. It is widely accepted that this capability depends largely on the performance of its scanning mechanism. Therefore, the design of scanning mechanism is always a key aspect of SPM development. Almost all of the SPM scanning mechanisms use piezoelectric ceramic electromechanical actuators to provide motions along scanning axes. Although it is not possible to describe all of the scanning mechanisms in this thesis, some of the moie common mechanisms will be discussed here. 


\subsubsection{Piezoelectric tripod}

One of the earliest three dimensional scanners used for scanning microscopy was that developed by Young et al. [5] for their Topografiner. A sample holder is supported by a flexible post which is driven vertically by a $z$ piezoelectric element and deflected by two orthogonally arranged lateral piezoelectric elements to produce $x-y-z$ scanning motions at the holder. Later on, a more compact and practical tripod scanner was built by Binnig and his co-workers [57], consisting of three separated piezoelectric transducers in a tripod arrangement in which the three meet at a common vertex on which the scanning tip is held, as shown in Fig. 2.11. It demonstrated a high resonant frequency and was found to be capable of scanning with high resolution. However, a scanning range of only a few micrometers was achieved because the scanning range is solely determined by the axial expansion of the piezoelectric elements. Drake et al. [58] used combinations of bimorphs in their designs of scanning mechanism to increase motion range and resonant frequencies.

\subsubsection{Tube scanner}

One of the replacements for tripod driver is tube scanner, which was first presented for this application by Binnig and Smith [59] in their STM and has become very popular in todays SPM systems. It is a three dimensional scanner fabricated from a single piezoelectric tube. The electrodes are coated on both the inside and outside walls. The electrode on the outside is axially sectioned into a quartered pattern with equal area for each part, as shown in Fig. 2.12. Applying a voltage to single inside electrode causes the tube to expand uniformly along its axis and $z$ motion is obtained. To realize the motion in $x$ and $y$ axes, voltages are applied on electrodes spaced $90^{\circ}$ a part on the outside individually, causing the tube to bend perpendicular to its axis in two orthogonal directions. A scanning tip is mounted at the end of the tube scanner. This design exhibits a low cross talk and a high resonant frequency. Commonly used tube scanners have similar dimensions to 


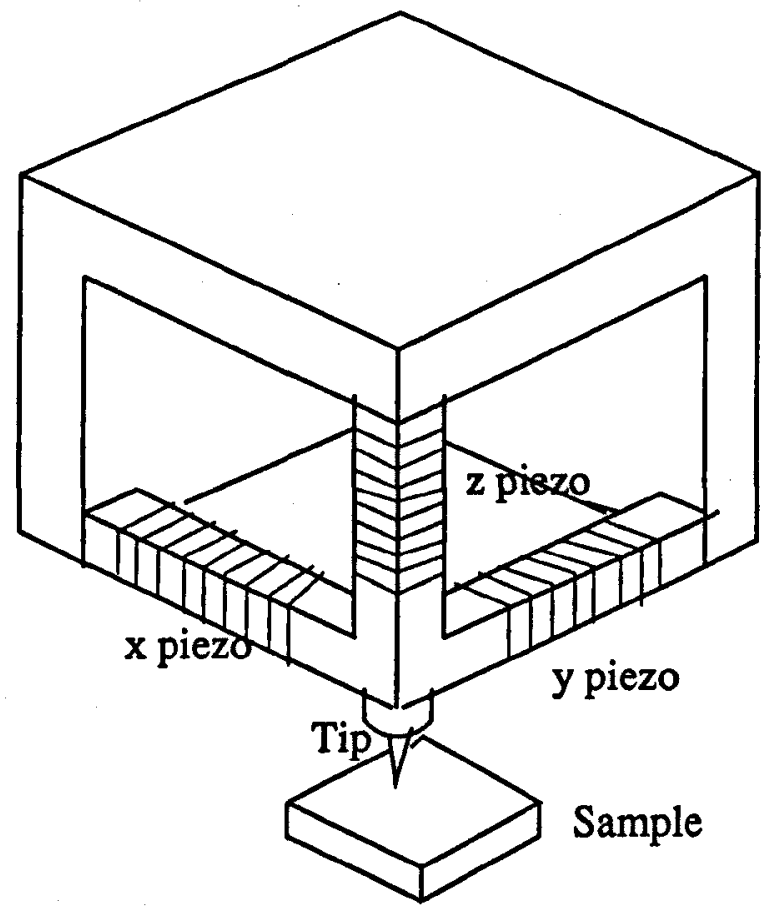

Figure 2.11: Schematic diagram of piezoelectric tripod scanning mechanism

that used by Binnig and Smith [59], which is measured $12.7 \mathrm{~mm}$ long, $6.35 \mathrm{~mm}$ in diameter and $0.51 \mathrm{~mm}$ thick, resulting in resonant frequencies of $8 k H_{z}$ in lateral and $40 \mathrm{kH}_{z}$ vertical. To improve the transient response behaviour, some users have filled the tube with silicon rubber [60] [61].

It should be: noted that the $x-y$ motion produced by simple tube bending is not entirely orthogonal to $z$ axis, which may reduce accuracy of tip position. The coupling can be minimized by mounting the probe tip on one of the dc motion electrodes [59]. By splitting the $x-y$ electrodes appropriately along $z$ axis, it is possible to benc: the tube into $S$ shape, as shown in Fig. 2.13. The end surface of the tube moves strictly parallel to the $x-y$ plane. Then the error can, therefore, be reduced. Again, the scanning range of the tube scanner is very small, usually a few micrometers. Although longer tubes can provide larger scanning range, a $100 \mathrm{~mm}$ tube for a $100 \mu \mathrm{m}$ expansion, as the tube becomes longer, its resonant frequency will reduce, theoretically with the inverse cube of the length, and the 
problem of creep and hysteresis will also become more serious.

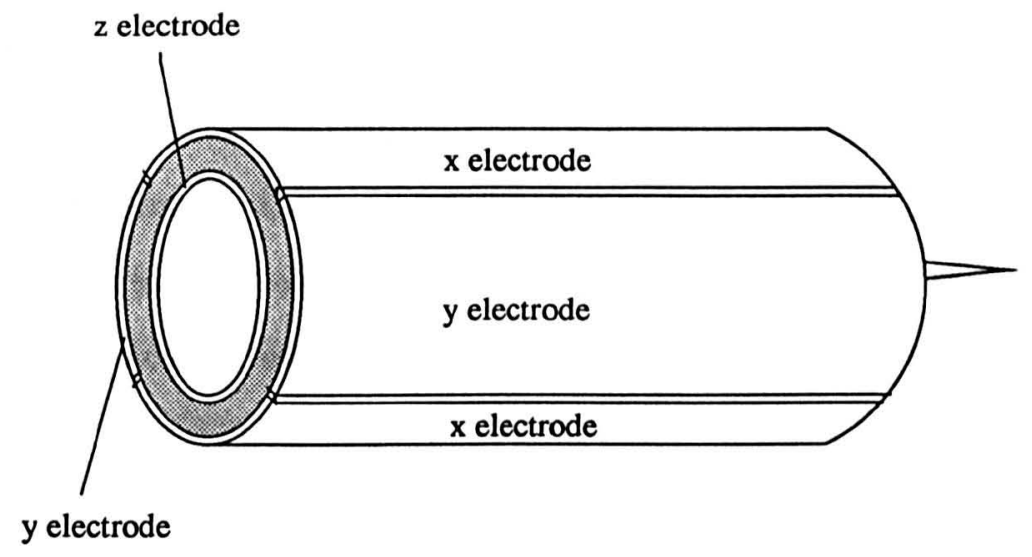

Figure 2.12: Schematic diagram of tube scanner

(a)

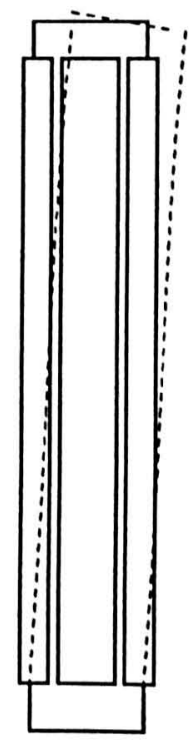

(b)

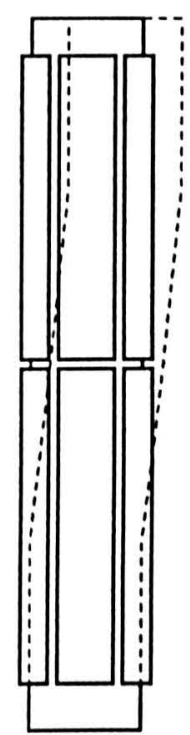

Figure 2.13: Bending mode of tube scanner. (a) Simple bending, (b) S-shape bending

\subsubsection{Bimorph scanner}

Similar to tube scanner in principle, piezoelectric bimorph can be used as scanning actuator. It consists of two thin sheets of piezo ceramic material, one of 
which expands and the other contracts upon application of a voltage, resulting in a bending motion. A bimorph x-y scanner built by Durig et al. 1985 [14] is schematically shown in Fig. 2.14. In this scanner, the sample holder is supported by a pair of parallel bimorphs which provide scanning motion in the $\mathrm{x}$ axis. The $y$-axis scanning is realized by adding another pair of parallel bimorphs with one end of them fixed to the base and the other end to the x-scanning mechanism. a scanning range of $50 \mu \times 50 \mu$ has been obtained.

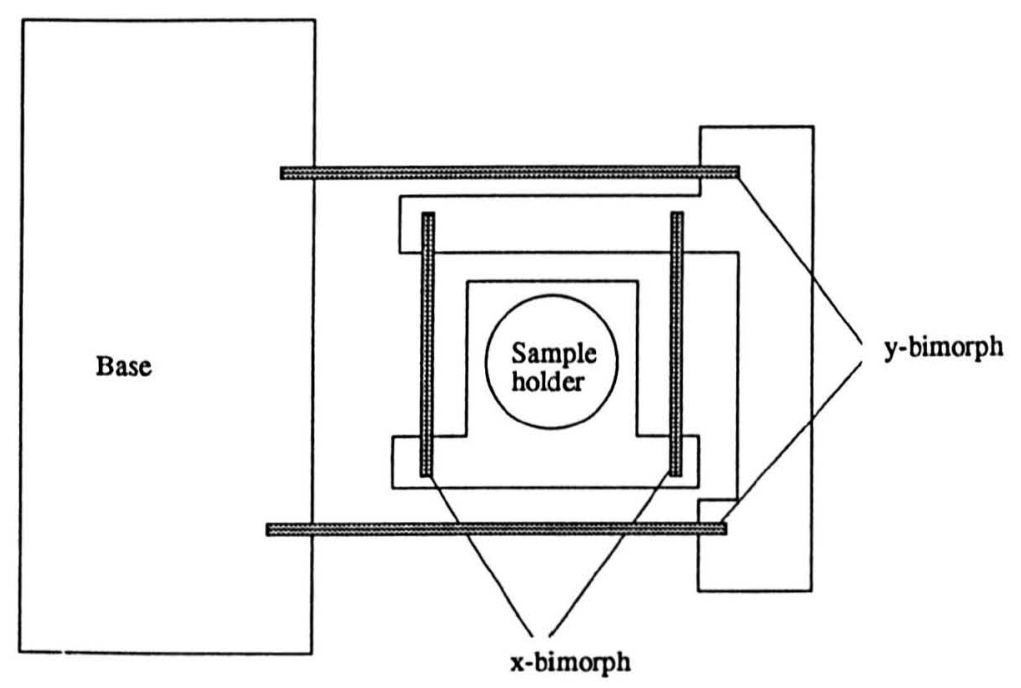

Figure 2.14: Schematic diagram of bimorphscanner

\subsubsection{Piezo-flexure stage}

In an effort to develop long range, metrological scanning microscopes, more and more precision scanning stages have been developed in recent years. At the time of writing, almost all of the scanning stages used in SPMs are piezo-driven flexure stages. This type of stage has the advantages of smooth motion (free from hysteresis and stick-slip friction), favourable dynamic properties and long time stability. Most of these stages are fabricated monolithically on a thick metal plate, usually aluminium alloys or stainless steel, in which lever arms and spring hinges are arranged to amplify the motions produced by the expansions of piezo actuators 
so that large motions can be obtained with a compact mechanism. For metrological purposes, a highly sensitive sensor can be easily inserted into the stage for position monitoring and/or closed loop control. The piezo-flex stage built by Scire and Teague (1978) [3] is probably the first precision stage of this type reported and is reproduced in Fig. 2.15. It is of a typical hinge-lever structure with notch hinges employed to produce flexure. $50 \mu \mathrm{m}$ range and subnanometer resolution were achieved, but its low resonant frequency and poor linearity limited its wide applications. The revised version of the stage was used in scanning tunneling microscopy by Fu et al. [63]. After that, various piezo-flexure scanning stages have been developed and many of them have been commercialized. Now the application of scanning stage in scanning microscopy has been increasingly reported, especially for metrological SPMs. [12] [65] [12]

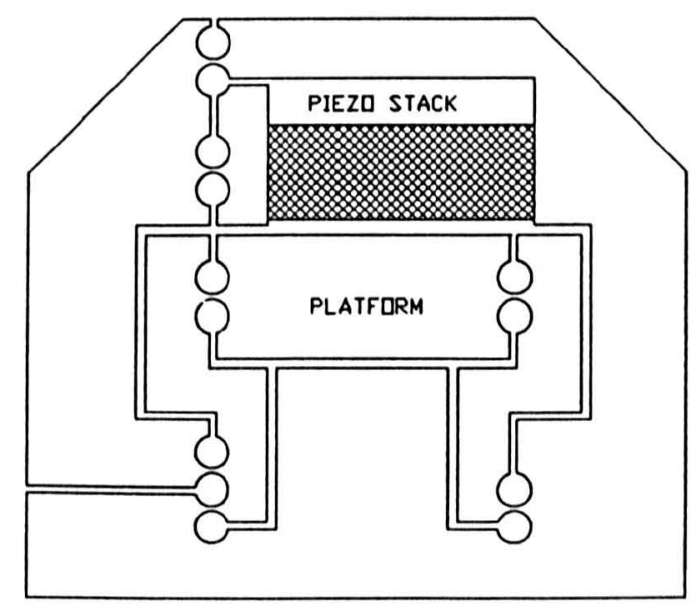

Figure 2.15: Schematic diagram of piezo-flexure stage, (From reference 59)

\subsubsection{Coarse positioning mechanism}

Coarse positioning generally refers to three dimensional relative motion of a probe tip to a sample, with a large moving range which covers the scanning range. 
Mostly concerned and investigated by designers is $z$ translating device which is usually called tip approaching mechanism. The tip approaching mechanism is to bring the tip approach to the sample to a proximity at which the interaction between them becomes available for detection system to pick up without punching the tip into the sample, and to maintain this in the controlling range of $z$ piezo. Therefore, the fundamental requirements for the mechanism comes to a smooth motion, a large positioning range and a relative high resolution.

\subsubsection{Inchworm}

Long range piezo-driven translators are widely used for coarse positioning in SPM. The "Inchworm" ${ }^{T M "}$ is a typical piezo-driven device available for this purpose. It combines piezo clamps and piezo expansion to produce step motion by properly implementing a sequence of clamping, unclamping, expanding and contracting. Fig. 2.16 shows schematically the principle of operation of the inchworm.

With this kind of device, a resolution of a few nanometers has been achieved and the translation range is theoretically unlimited [60][65]. However, the effects of clamping introduce parasitic motions typically of many 100's of nanometers. As a consequence they can only be used to provide the motions of resolutions that are between a clamp cycle.

\subsubsection{Piezo-walker}

Another well known piezo driven device is micro-walker, so called "louse", which was firstly developed by Binnig et al. [1]. A typical design of this type is shown in Fig. 2.17. The walker consists of a piezoplate and three dielectric feet standing on a metal plate. The feet can glide freely on the metal plate or are clamped in place by applying a voltage on them. Two dimensional motion can be realized by expansion and contraction of the piezoplate with an appropriate clamping sequence of the feet. A resolution of a few nanometers and a dynamic range of centimeters have been obtained. The drawback of the device is that the clamping 
(a)

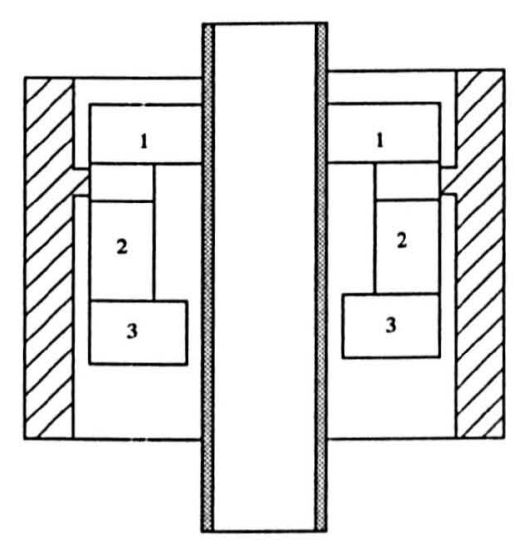

(c)

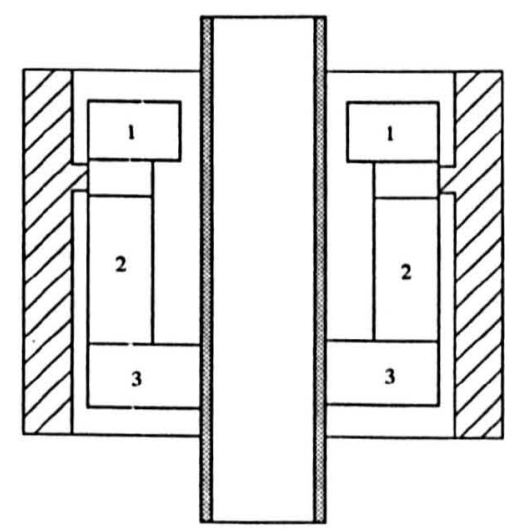

(b)

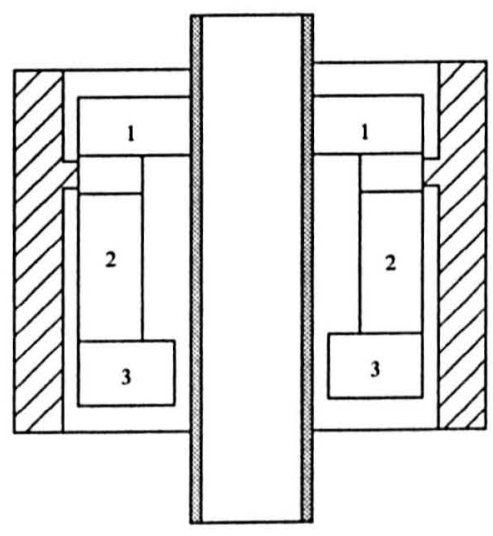

(d)

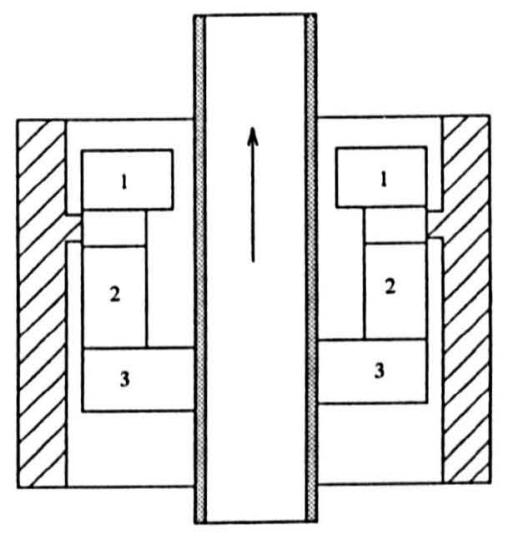

Figure 2.16: Operation principle of inchworm. (a) The inchworm is ready for a new step. piezo 1 clamps. (b) Piezo 2 expands. (c) Piezo 3 clamps and Piezo 1 releases. (d) Piezo 2 contracts, and the tube is moving up. 
forces are very sensitive to the surface condition of the dielectric material and poor in low resonant frequency. Decreased piezo expansion at low temperature also makes the louse performance unreliable for low-temperature application.

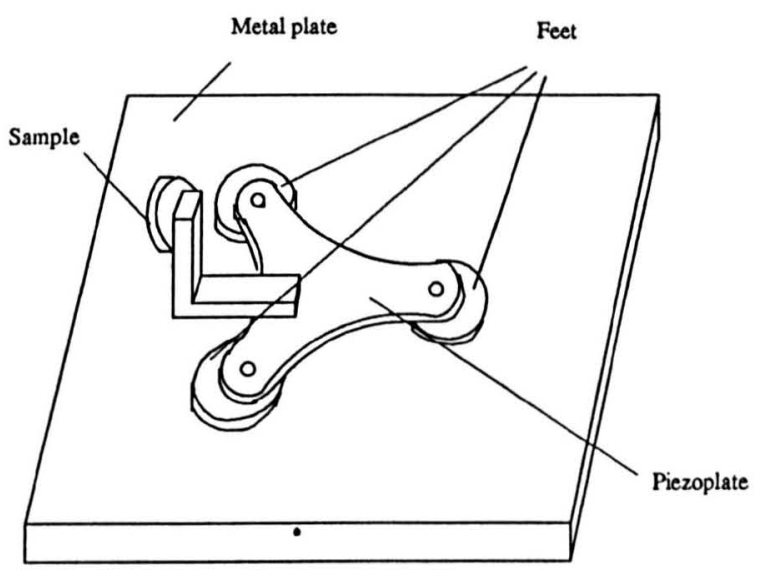

Figure 2.17: Schematic diagram of operation principle of piezo-walker

\subsubsection{Magnetic walker}

To overcome the problems on piezo-louse mentioned above, a magnetic walker was first built at Stanford University [67] and used by Elrod et al.[68] for lowtemperature vacuum tunneling microscopy. It was later developed and employed for STM by other groups [69][70]. The device generates movement from the force derived from pulses of current in the presence of a magnetic field. It slides on a low friction surface and requires no clamping, as shown schematically in Fig. 2.18. A permanent magnet is mounted on a sample holder which sits on a plate of smooth surface through three legs. A solenoid is fixed with the plate. When current is pulsed through the coils, an induced force pushes the sample holder moving in steps. The holder can move backward and forward depending on the direction of the current pulses. Again nanometer resolution over centimeter motions have been achieved. If two pairs of permanent magnets and solenoids are properly ar:anged, two dimensional motion can also be achieved. 


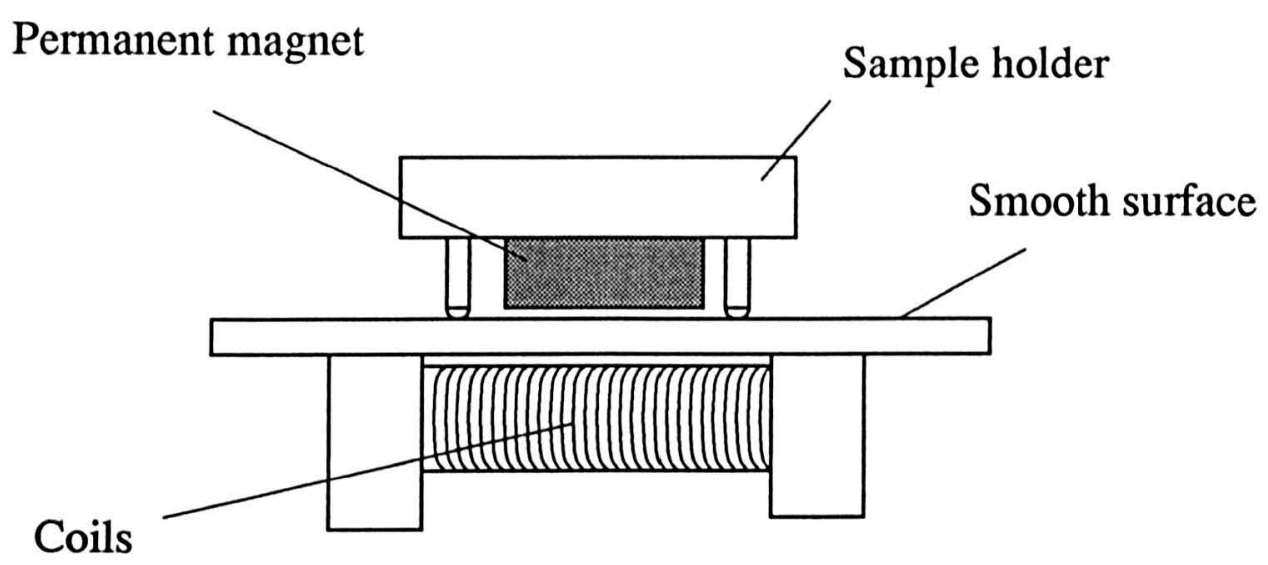

Figure 2.18: Schematic diagram of magnetic walker

\subsubsection{Inertial glider}

Built by Pohl [71] an inertial glider is another piezo-driven positioning device successfully used for tip approaching in scanning probe microscopy. As shown in Fig. 2.19, it consists a sample holder riding on a guide-way which is supported by a pair of leaf springs. The leaf springs connect the guide-way to a base and a piezo tube is firmly clamped between the guide-way and the base. A sawtooth electric waveform is applied to the piezo tube to generate step motion. During the slow ramp piezo's expansion causes guide-way and sample holder moving forwards together due to static friction forces. While the rapid jump of piezo's contract produces such a high acceleration of guide-way that the holder does not follow it anymore because of its inertial forces. Then a sliding motion happens between them. The device provided step sizes of $0.04-0.2 \mu \mathrm{m}$ and translation range is only limited by its structure.

Anders et al. [72] built an inertial sliding positioner capable of two dimensional motion. In their design a piezoelectric disc is clamped into a quadrate metal frame with a sample holder siting on it. The electrode on one side of the disc is cut into four quadrants. Step motion in disc plan is achieved by connecting different neighbouring pairs of quadrant electrodes to the same sawtooth-shaped 


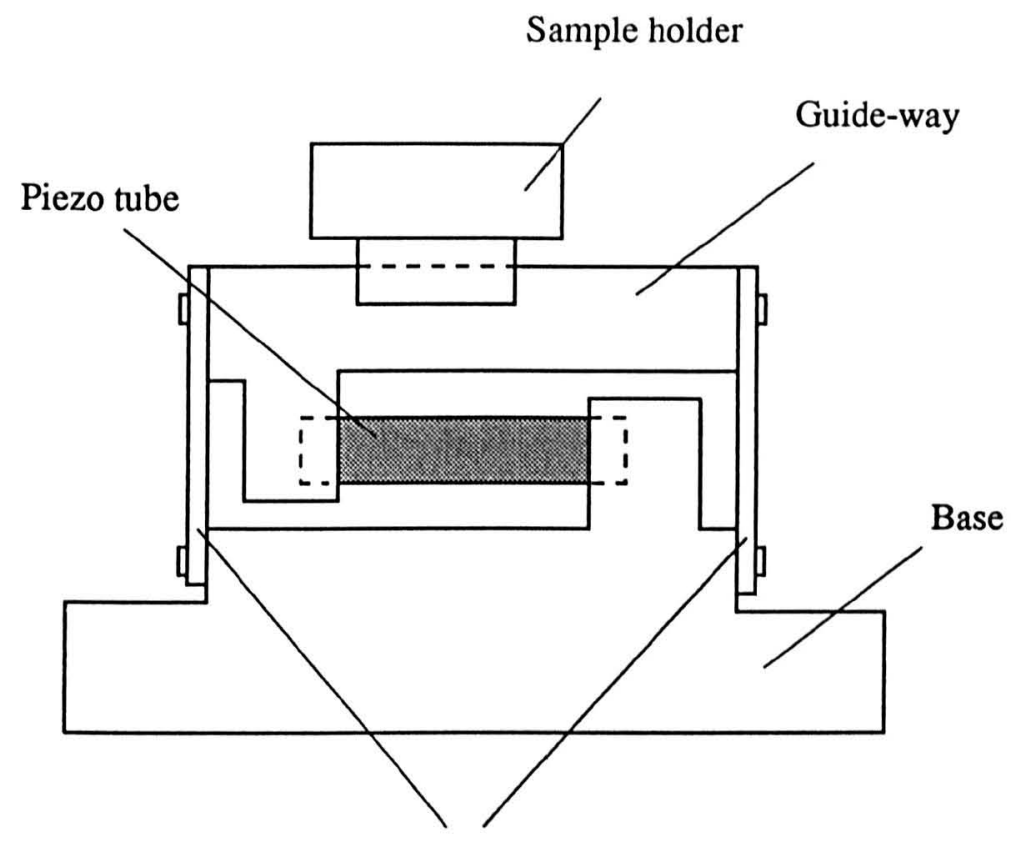

Leaf-spring

Figure 2.19: Schematic diagram of inertial glider

voltage. The inertial forces generate relative motion between the disc and the holder.

\subsubsection{Scre'd-driven mechanism}

Screw-driven mechanisms have also been widely explored and possibly provide the least expensive method for tip/specimen adjustment. Differential micrometers can be used directly for tip approach [73]. Various lever reduced and differential spring reduced screw mechanisms have also been developed and used for this purpose [74][75j] [76][77]. As an example, for one such mechanism, motion of a lead screw turned by rotational manipulator is reduced by a lever with a ratio of $1 / 100-1 / 5000$, resulting in a $500 \stackrel{\circ}{A}$ to $25 \mu \mathrm{m}$ translation per revolution [74]. Similarly, for differential spring mechanism the reduction of motion is produced by difference in spring constants between two series arranged springs. Fig. 2.20 shows a simple differential spring mechanism [4]. 


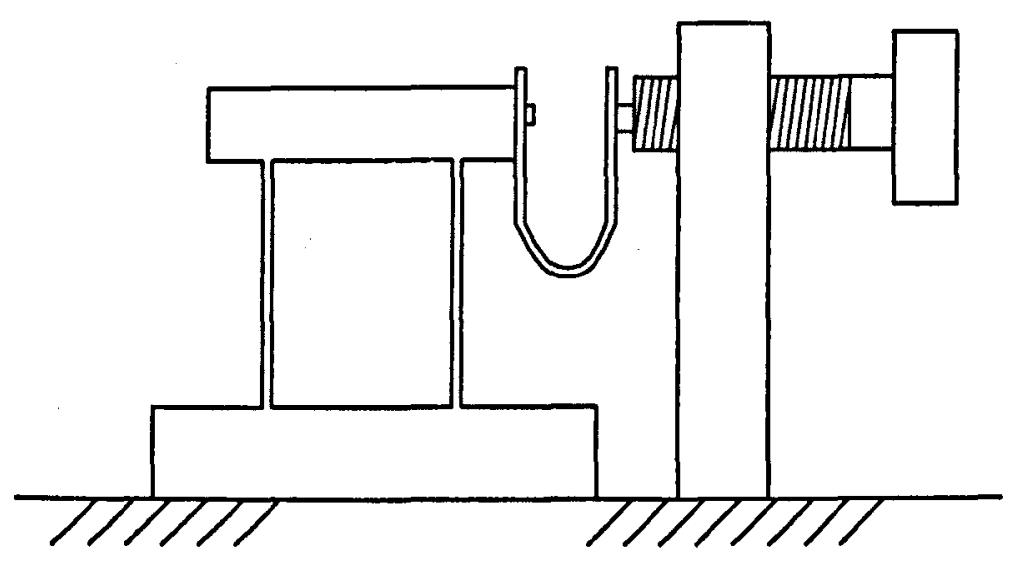

Figure 2.20: Schematic diagram of a differential spring reduced screw positioner 


\section{Bibliography}

[1] G. Binnig, H. Rohrer, Ch. Gerber, and E. Weibel, 1982, Tunneling through a controllable vacuum gap, Phys. Rev. Lett., 40178 - 182

[2] G. Binnig and H. Rohrer, 1982, Scanning tunneling microscopy, Helvetica Physica Acta 55, 726 - 735

[3] Y. Kuk and P.J. Silverman, 1989, Scanning tunneling microscope instrumentation, Rev. Sci. Instrum. 60(2), $165-180$

[4] R.D. Young, 1966, Field emission ultramicrometer, Rev. Sci. Instrum. 37(3), $275-278$

[5] R. Young, J. Ward, and F. Scire, 1972, The topografiner: an instrument for measuring surface microtopogrphy, Rev. Sci. Instrum. 43(7), 999 - 1011

[6] G. Binnig, H. Rohrer, Ch. Gerber, and E. Weibel, 1983, $7 \times 7$ reconstruction on $\mathrm{Si}(111)$ resolved in real space,Phys. Rev. Lett., 50120 - 123

[7] G. Binnig, C.F. Quate and C.G. Gerber, 1986, Atomic force microscope, Phys. Rev. Lett., 56 (9) 930 - 933

[8] T.R. Albrecht, and C.F. Quate, 1988, Atomic resolution with the atomic force microscope on conductors and nonconductors, J. Vac. Sci. Technol., A 6, 271 - 274

[9] D. Sarid, 1991, Scanning force microscopy with applications to electric, magnetic, and atomic forces, Oxford University Press 
[10] D. Sarid, 1991, Review of scanning force microscopy, J. Vac. Sci. Technol. , B 52 (2), $431-437$

[11] Lee Davis, private communication.

[12] Y. Martin and H.K. Wickramasinghe, 1987, Magnetic imaging by "force microscopy, with $1000 \AA$ r resolution, Appl. Phys. Lett. 50 (20) $1455-1457$

[13] Y. Martin, D.W. Abraham, and H.K. Wickramasinghe, 1988, Highresolution capacitance measurement and potentiometry by force microscopy, Appl. Phys. Lett. 52 (13), 1103 - 1105

[14] U. Durig, D. Pohl, and F. Rohrer, 1986, Near-field optical-scanning microscopy, J. Appl. Phys. 59(10), 3318 - 3327

[15] M. Isaacson, J. Cline and H. Barshatzky, 1991, Near-field-optical microscopy, Scanned Probe microscopy, Santa Barbara, CA 1991, edited by H.K. Wickramasinghe, American Institute of Physics, New York, 23 - 36

[16] J.R. Matey and J. Blanc, 1985, Scanning capacitance microscopy, J. Appl. Phys. 57(5), 1497 - 1444

[17] C.D. Bugg and P.J. King, 1988, Scanning capacitance microscopy, J. Phys. E21 $147-151$

[18] C.C. Williams, W.P. Hough, and S.A. Rishton, 1989, Scanning capacitance microscopy on a $25 \mathrm{~nm}$ scale, Appl. Phys. Lett. 55(2), 203 - 205

[19] C.C. Willi.ums and H.K. Wickramasinghe, 1986, Scanning thermal profiler, Appl. Phys. Lett. 49(23), 1587 - 1589

[20] J.M.R. Weaver, L.M. Walpite, and H.K. Wickramasinghe, 1989, Optical absorption microscopy and spectroscopy with nanometer resolution, Nature, 342, 783 - 785 
[21] M. Nonnenmacher and H.K. Wickramasinghe, 1992, Scanning probe microscopy of thermal conductivity and subsurface properties, Appl. Phys. Lett., $61(2), 168$ - 170

[22] A. Majumdar, J.P. Carrejo, and J. Lai, 1993, Thermal imaging using the atomic force microscope, Appl. Phys. Lett. 62(20), 2501 - 2503

[23] R.J. Pylkki, P.J. Moyer and P.E. West, 1994, Scanning near-field optical microscopy and scanning thermal microscopy, Jpn. J. Appl. Phys., 33, 3785 $-3790$

[24] P.K. Hamsina, B. Drake, O. Marti, S.A.C. Gould, and C.B. Prater, 1989, The scanning ion-conductance microscope Science, 243, $641-649$

[25] G. Binnig, Ch. Gerber, E. Stoll, T.R. Alrecht, and C.F. Quate, 1987, Atomic resolution with atomic force microscope, Europhys. Lett. 3, $1281-1286$

[26] O. Marti, B. Drake, and P.K. Hansma, 1987, Atomic force microscopy of liquid-covered surface: Atomic resolution images, Appl. Phys. Lett. 51, 484 $-486$

[27] M.D. Kirk, T.R. Albrecht, and C.F. Quate, 1988, Low-temperature atomic force microscopy, Rev. Sci. Instrum. 59, 839 - 835

[28] T. Goddenhenrich, H. Lemke, U. Hartmann, and C. Heiden, 1990, Force microscope with capacitance displacement detection, J. Vac. Sci. Technol., A 8 (1), $383-387$

[29] G. Neubauer, S.R. Cohen, G.M. McClelland, D. Home and C.M. Mate, 1990, Force microscopy with a bidirectional capacitance sensor, Rev. Sci. Instrum., 61 (9), 2296 - 2308

[30] S.T. Joycce and J.E. Houston, 1991, A new force sensor incorporating forcefeedback control for interfacial force microscopy, Rev. Sci. Instrum., 62 (3), $710-715$ 
[31] G.L. Miller, J.E. Griffith, and E.R. Wagner, 1991, A rocking beam electrostatic balance for the measurement of small forces, Rev. Sci. Instrum., 61 (9), $705-709$

[32] G. Meryer, N.M. Amer, 1988, Novel Optical approach to atomic force microscopy, Appl. Phys. Lett. 531045 - 1047

[33] G. Meryer, N.M. Amer, 1988, A simple optical method for the remote sensing of stylus deflection in atomic force microscopy, Bull. Amer. Phys. Soc. 33, 319

[34] C.A.J. Putman, B.G. De Grooth, N.F. Van Hulst, and J. Greve, 1992, A detailed aralysis of the optical beam deflection technique for use in atomic force microscopy, J. Appl. Phys. 72 (1), 6 - 12

[35] G. Meryer, N.M. Amer, 1990, Optical-beam-deflection atomic force microscopy: The $\mathrm{HaCl}$ (001) surface, Appl. Phys. Lett. 56 (21), $2100-2101$

[36] S.Alexander, L. Hellemans, O. Marti, J. Schneir, V. Elings, and P.K. Hansma, M. Longmire and J. Gurley, 1989, An atomic-resolution atomicforce microscope implemented using an optical lever, J. Appl. Phys. 65 (1), $164-167$

[37] M.A. Taubenblatt, 1989, Lateral forces and topography using scanning tunneling microscopy with optical sensing of the tip position, Appl. Phys. Lett. 54801

[38] L.A. Wenzler, T.Han, R.S.Bryner, and T.P. Beebe, Jr., 1994, An integrated scanning tunneling, atomic force and lateral force microscope, Rev. Sci. Instrum., 65 (1), $85-88$

[39] D. Sarid, D.A. Iams and V. Weissenberger, 1988, Compact scanning force microscope using a laser diode, Opt. lett., 13 1057 - 1059 
[40] D. Sarid, V. Weissenberger, D.A. Iams and J.T. Ingle, 1989, Theory of the laser diode interaction in scanning force microscope, IEEE J. Q. Elec., 25(8), $1968-1972$

[41] D. Sarid, D.A. Iams, J.T. Ingle, V. Weissenberger, and J. Ploetz, 1990, Performarce of a scanning force microscope using a laser diode, J. Vac. Sci. Technol., A 8 (1), 378 - 382

[42] D. Sarid, P. Pax, L. Yi, S. Howells, M. Gallagher, T. Chen, V. Elings and D. Bocek, 1992, Improved atomic force microscope using a laser diode interferometer. Rev. Sci. Instrum., (33(8), 3905 - 3908

[43] G.M. Mcclelland, R. Erlandsson, and S. Chiang, 1987, Atomic force microscopy: ieneral principles and a new implementation, Review of Progress in Quanti! ttive Non-Destructive Evaluation, edited by D.O. Thompson and D.E. Chimenti, Plenum, New York, Vol. 6B, 1907 - 1314

[44] R. Erland šen, G.M. McClelland, C.M. Mate, and S. Chiang, 1988, Atomic force microscopy using optical interferometry, J. Vac. Sci. Technol., A6(2), $266-270$

[45] D. Rugar. H.J. Mamin, R. Erlandssen, J.E. Stern, and B.D. 'Terris, 1988, Force microscope using a fiber-optic displacement sensor, Rev. Sci. Instrum., $59(11), 2337$ - 2340

[46] D. Rugar, H.J. Mamin, and P. Guethner, 1989, Improved fiber-optic interferometer jor atomic microscopy, Appl. Phys. Lett. 55 (25), $2588-2590$

[47] S. Breen, 3.E. Blackford, and M.H. Jericho, 1990, Fiber optical displacement sensor witl subangstrom resolution, Appl. Opt. 29(1), $16-18$

[48] P.J. Mulh:rn, T. Hubbard, C.S. Arnold, B.L. Blackford and M.H. Jericho, 1991, A scinning force microscope with a fiber-optic-interferometer displacement sensı r, Rev. Sci. Instrum., 62(5), 1280 - 1284 
[49] A.J. den Boef, 1989, Scanning force microscopy using a simple low-noise interferometer, Appl. Phys. Lett. 55(5), 439 - 441

[50] C. Schonenberger and S.F. Alvarado, 1989, A differential interferometer for force microscopy, Rev. Sci. Instrum., 60(10), 3131 - 3134

[51] Y. Martin, C.C. Williams, and H.K. Wickramasinghe, 1987, Atomic force microscope-force mapping and profiling on a sub $100-\AA$ scale, J. Appl. Phys. 61(10), 4723 - 4731

[52] Y. Martin and H.K. Wickramasinghe, 1987, Magnetic imaging by "force microscopy" with $1000 \AA$ resolution, Appl. Phys. Lett. 50(20), 1455 - 1457

[53] Y. Martin, D. Rugar, and H.K. Wickramasinghe, 1988, High-resolution magnetic imaging of domains in $T_{b} F_{e}$ by force microscopy, Appl. Phys. Lett. $52(13), 244246$

[54] Y. Martin, D.W. Abraham, and H.K. Wickramasinghe, 1988, High resolution capacitance measurement and potentiometry by force microscopy, Appl. Phys. Lett. 52(13), 1103 - 1105

[55] T. Itoh and T. Suga, 1993, Development of a force sensor for atomic force microscopy using piezoelectric thin films, Nanotechnology, 4, $218-224$

[56] L.P. Howard, 1993, Advances in ultra-low contact force nanometric surface metrology, Ph.D Thesis, Unversity of Warwick

[57] G. Binnig, H.Rohrer, Ch. Gerber, and E. Weibel, 1982, Surface studies by tunneling microscopy, Phys. Rev. Lett. 49(1), 57 - 61

[58] B. Drake, R. Sonnenfeld, J. Schneir, P.K. Hamsma, G. Slough and R.V. Coleman, 1986, Tunneling microscope for operation in air or fluids, Rev. Sci. Instrum., $\mathbf{5 7}(3), 441-445$ 
[59] G. Binnig and D.P.E. Smith, 1986, Single-tube three-dimensional scanner for scanning tunneling microscopy, Rev. Sci. Instrum.,57(8), 1688 - 1689

[60] H. Olin, 1994, Design of a scanning probe microscope, Meas. Sci. Technol. 5, $976-984$

[61] D.P. DiLella, J.H. Wandass, R.J. Colton and C.R.K. Marrian, 1989, Controlsystems for scanning tunneling microscope Rev. Sci. Instrum., 60(6), 997 1002

[62] F.E. Scire and E.C. Teague, 1978, Piezo driven $50-\mu \mathrm{m}$ range stage with subnanometer resolution, Rev. Sci. Instrum., 49(12), $1735-1740$

[63] J. Fu, R.D. Young, and T.V. Vorburger, 1992, Long-range scanning for scanning tunneling microscopy, Rev. Sci. Instrum., 63(4), 2200-2205

[64] Y. Xu, S.T. Smith, P.D. Atherton, T. Judge and R. Jones, 1994, A metrological scanning force microscope, Proceedings of ASPE 9th annual meeting, Cincinnati, $23-28$

[65] O. Jusko, X. Zhao, H. Wolff, and G. Wilkening, 1994, Design and three dimensional calibration of a measuring scanning tunneling microscope for metrological applications, Rev. Sci. Instrum., 65(8), 2514-2518

[66] T.H. McWaid and J. Schneir, 1994, The design of an atomic force microscope for metrology, Proceedings of ASPE 9th annual meeting, Cincinnati, 174 177

[67] D.P.E. Smith and S.A. Elrod, 1985, Magnetically driven micropositioners, Rev. Sci. Instrum., 56(10), 19701971

[68] S.A. Elrod, A.L. de Lozanne, and C.F. Quate, 1984, Low-temperature vacuum tunneling microscopy, Appl. Phys. Lett. 45(11), $1240-1242$ 
[69] B.W.Corb, M. Ringger, and H.J. Guntherodt, 1985, An electromagnetic microscopic positioning device for the scanning tunneling microscope, $\mathrm{J}$. Appl. Phys. 58(11), 3947 - 3953

[70] K. Kajimura, H. Bando, K. Endo, W. Mizutani, H. Murakami, M. Okano, S. Okayama, M. Ono, Y. Ono, H. Tokumoto, F. Sakai, K. Watanabe and S. Wakiyama, 1987, Construction of an STM and observation of $2 \mathrm{H}-\mathrm{NbSe}_{2}$ atomic images, Surface Science 181, 165 - 173

[71] D.W. Pohl, 1987, Dynamic piezoelectric translation devices, Rev. Sci. Instrum., 58(1), 5 \& - 57

[72] M. Anders, M. Thaer and C. Heiden, 1987, A simple micropositioning devices for STM, Surface Science 181, 176 - 182

[73] H. Bando, N. Morita, H. Tokumoto, W. Mizutani, K. Watanabe, A. Homma, S. Wakiyama, M. Shigeno, K. Endo, and K. Kajimura, 1988, Scanning tunneling spectroscopy study on graphite and $2 \mathrm{H}_{-} \mathrm{NbSe}_{2}$, J. Vac. Sci. Technol., A6 (2), $344-348$

[74] J.E. Demuth, R.J. Hamers, R.M. Tromp, and M.E. Welland, 1986, A scanning tunneling microscope for surface studies, IBM J. Res. Dev. 30, 397 402

[75] M.P. Cox and P.R. Griffin, 1988, A high-performance scanning tunneling microscope, J. Vac. Sci. Technol., A6(2), $376-378$

[76] A.P. Fein, J.R. Kertley, and R.M. Feenstra, 1987, Scanning tunneling microscope for low temperature. high magnetic field, and spatially resolved spectroscopy, Rev. Sci. Instrum., 58(10), 1806 - 1810

[77] P. Davidsson, T. Claeson, and S. Pehrson, 1988, A new symmetric scanning tunneling microscope design, J. Vac. Sci. Technol., A6, 380 - 382 
[78] S.T.Smith and D.G. Chetwynd, 1992, Ultra precision mechanism design, Gordon and Breach Sci. Publishers 


\section{Chapter 3}

\section{Force probe system}

\subsection{Introduction}

The force probe is the heart of a scanning force microscope. Its force sensing capability and other properties have a direct effect on the performance of the microscope. From a general point of view, almost all the force probes developed so far measure deflections of a cantilever as the force sensing mechanism. A sharp tip is mounted on a flexible cantilever beam whose deflection senses the resultant interaction of the tip with the surface. The role of the beam is to transfer the force acting on the cip into a deflection that can subsequently be monitored by various deflection-sensing methods. Among these, electron tunneling, capacitance, piezoelectricity, piezoresistivity, optical interferometry, optical polarization, and optical deflection have been used. A nanometre or sometimes even subnanometre resolution can be achieved by these techniques.

The force probe introduced in this chapter is a capacitance based constant force sensor, the first prototype of which was built and used by L.P.Howard and S.T.Smith $[1,2,3]$ in our laboratory. It demonstrated a very good sensitivity and a high stability. Further understanding of the characteristics and the optimization of the force probe design was investigated by the author. Then the new optimized force probe was applied to a metrological scanning force microscope. 
Fig. 3.1 shows a diagram of the force probe sensor. The probe consists of a thin cantilever beam that is rigidly attached to a solid base at one end and with the stylus tip attached at the other. The base extends below the cantilever on the face opposite to the tip and is separated by a small air gap. An aluminium film deposited onto the opposing faces forms capacitance electrodes which are used to monitor the deflection of the beam.

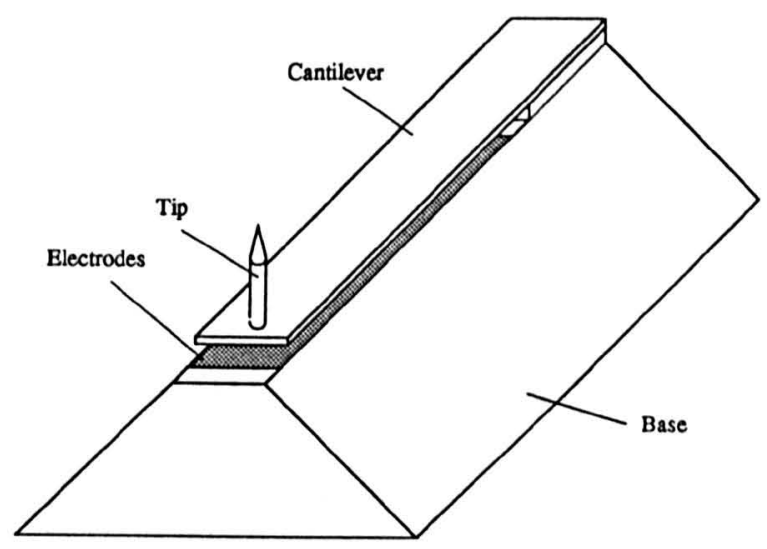

Figure 3.1: The force probe

Generally, the higher the resolution of the instrument, the smaller the field of view or measurement length. Although AFM is capable of very high resolution, its scanning range is too small for application to many precision engineering problems. Typically, traditional stylus instruments can measure profiles over many millimeters, but are limited to a relative low traverse speed and high contact forces. The constant force probe presented in this thesis is aimed to be developed as a hybrid of these techniques incorporating both the long range traverse characteristic of stylus instruments and high bandwidth response and low contact force of AFM. Theoretically, an ideal probe should be made as small as possible to reduce mass with minimal separation and large area electrodes for high sensitivity. However, as the separation reduces there arises significant squeeze film forces which, for small oscillations, result in an inherent viscous type damping between the beam and the base. The parameters of the cantilever beam not only 
determine the electrode area, but also affect the probe's dynamic properties as well. Sometimes, these effects are contradictory, so great care has to be taken in balancing the various aspects properly if an optimal probe is to be built.

In this chapter, a theoretical analysis of the dynamic characteristics of this kind of force probe is introduced and extended to an assessment of the sensor's characteristics. Based on this analysis. optimization is discussed and methods for parameters selection are presented. The experimental verification of squeeze film forces between the beam and base is demonstrated and other properties of the probe have also been assessed experimentally. Finally, fabrication consideration are briefly discussed.

\subsection{Effects of damping on surface measurement with stylus method}

The scanning force microscope is generally considered as a new generation of stylus technique. In particular, the contact mode SFM simulates the conventional stylus instrument in performance and many other aspects. As the surface features to be measured become finer and finer, the restrictions on the environmental conditions become more stringent. This requires modern instruments to speed up the measurement cycle so as to minimize the influence of environmental drift and vibration. In this case, the dynamic properties of the instrument become of increasing concern to both designers and users. A high natural resonant frequency provides high dynamic response which enables the tip to follow the sharp changes of contours while damping helps to reduce susceptibility to vibration and may iacrease general measuring fidelity. However, too much damping makes the dynamic response unacceptably slow, with a corresponding increase in tip/surface forces which may cause damage to the surface. Theoretical work on stylus dynarnics by Whitehouse $[4,5]$ based on a study of the reaction force at the contact indicates that the measurement fidelity might be enhanced and 
measuring speed be increased if the damping factor is controlled properly.

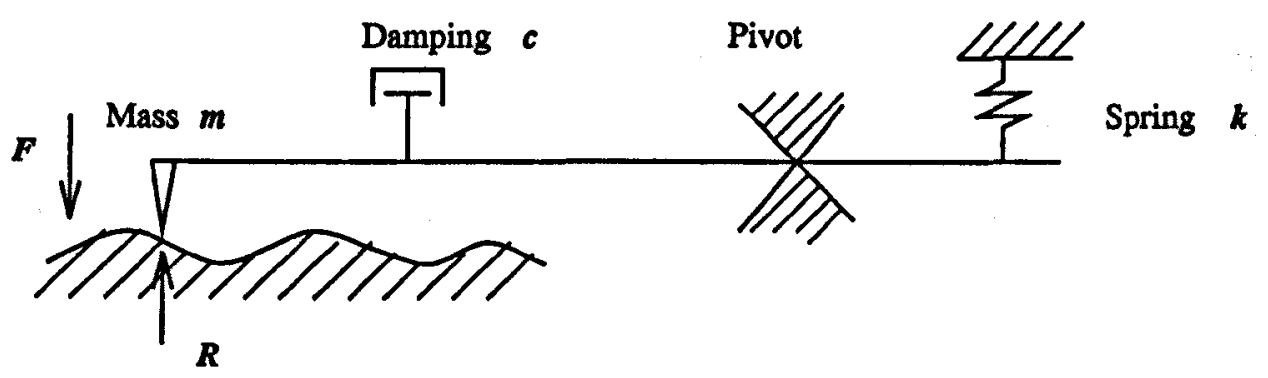

Figure 3.2: Schematic diagram of simplified model of general stylus method

For modeling purposes, a stylus system may be represented by a simple massspring-damping system, Fig. 3.2. The governing equation is

$$
m_{e} \ddot{y}+c \dot{y}+k y+F=R(t)
$$

where $m_{e}$ is the effective mass of the stylus relative to pivot, $c$ is the damping factor, $k$ is the rate of the equivalent spring, $F$ is the static force and $R$ is the reaction force. The statistical form of the model is more realistic for a discussion of the fidelity of surface measurement. From equation 3.1, taking the Fourier transform of both sides, squaring and taking to the limit gives $P_{R}(\omega)$, the power spectral density of $R(t)$

$$
P_{R}(\omega)=P_{Y}(\omega)\left[1+\left(\frac{\omega}{\omega_{n}}\right)^{2}\left(4 \xi^{2}-2\right)+\left(\frac{\omega}{\omega_{n}}\right)^{4}\right]
$$

The expression $\left[1+\left(\frac{\omega}{\omega_{n}}\right)^{2}\left(4 \xi^{2}-2\right)+\left(\frac{\omega}{\omega_{n}}\right)^{4}\right]$, designated as $H(\omega)$, is the weighting factor on $P_{Y}(\omega)$ to produce $P_{R}(\omega)$. From this equation it can be seen that the properties of the output $P_{R}(\omega)$ are related intimately to the system and input signal parameters, $m, \omega_{n}, \xi$, and $P_{Y}(\omega)$ respectively. From the point of view of damage and fidelity, two ways can be approached to improve the performance 
of the stylus instrument. One is to reduce the reaction; the other is to make the reaction match the surface profile. According to the equations 3.1 and 3.2 , reducing damping is an obvious way to reduce the magnitude of the reaction, but making $\xi$ tend to 0 introduces the problems of sensitivity to disturbances and long settling times which should be avoided in the instrument systems. However, too much damping makes the dynamic response unacceptably slow, with a corresponding increase in reaction $R$, which may cause damage and deteriorate fidelity. Therefore, there exists an optimum value of damping ratio $\xi$ to improve

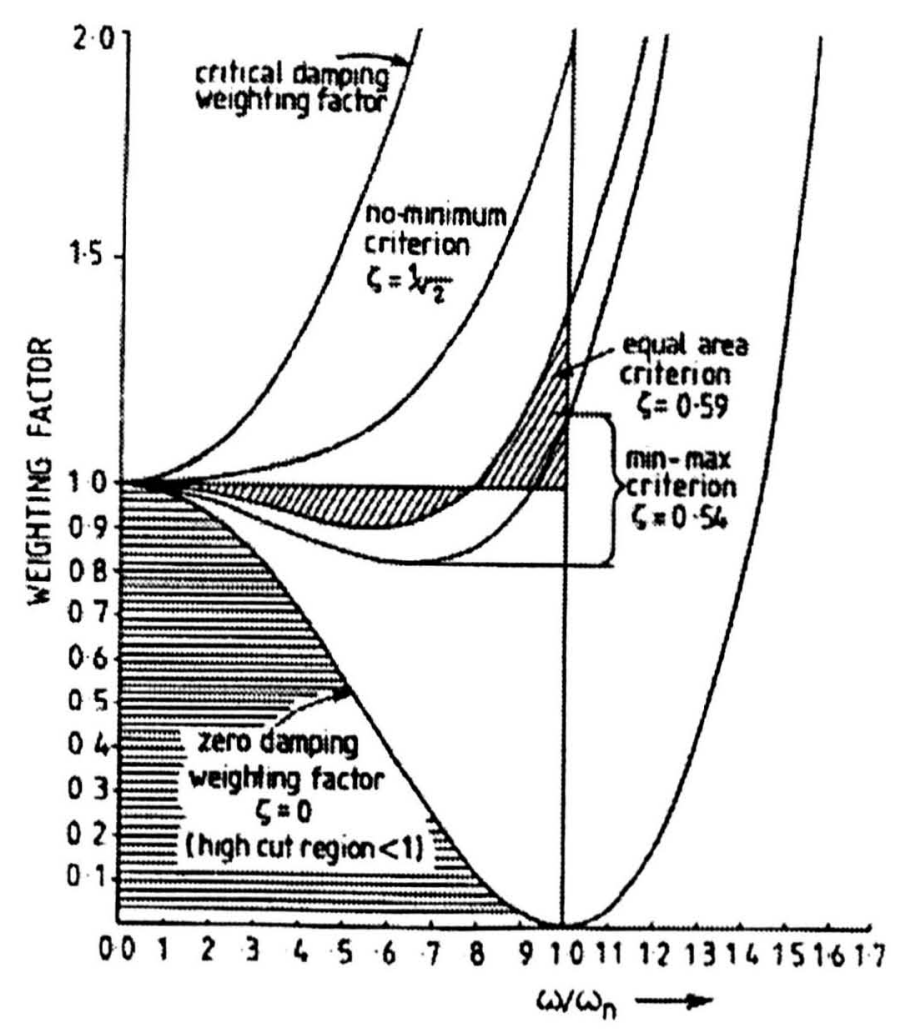

Figure 3.3: Surface spectrum weighting factors for different damping system (after reference 5)

fidelity and reduce damage. One approach is to optimize the shape of $H(\omega)$. Fig. 3.3 shows the shapes of weighting factor $H(\omega)$ corresponding to different damping systems. One criterion is to make $H(\omega)=1$ over the band of interest. The 
classical way to do this is to make $\omega_{n} \gg \omega_{u}\left(\omega_{u}\right.$ is the highest frequency on the surface), but it; is unrealistic for modern instruments because very fine features have to be measured and relative high speed is required by these instruments. An alternative criterion, which utilizes all the band of frequencies up to $\omega_{n}$, is to pick damping ratio $\xi$ from equation

$$
\int_{0}^{\omega_{n}} H(\omega) d \omega=1
$$

In this case, $H(\omega)$ is not unity for each individual value of $\omega$, but it is unity, on average, over the whole range. The solution for the equation (3.3) comes to be $\boldsymbol{\xi}=0.59$.

Damping effects on the fidelity of surface measurement have also been experimentally investigated by Liu et al.[6]. From this study damping ratios corresponding to optimal fidelity are claimed to be in the range of $0.4-0.8$.

\subsection{Dynamic characteristics of the force probe: theoretical analysis}

From the damping effects discussed above, it is clear that dynamic properties of the stylus instruments have direct influence on the surface measurement. It is easy to see that the force probe of cantilever type can be considered, in principle, as a miniature stylus system. It is generally used to measure the surface on much finer features with much lower contact force and higher speed. In this case, its dynamic characteristics become paramount factors for obtaining desired performance from the measurement system.

The geometric parameters for mathematical modeling are shown in figure 3.4. The cantilever of the force probe is modeled as a simple prismatic beam with concentrated mass (diamond tip) on the free end and distributed mass (beam mass) along the beam. Since the beam has distributed weight, theoretically, it 
can vibrate at an infinite number of frequencies, where each frequency has a unique deformation profile, or vibration mode. In reality it is the lowest, or fundamental, mode that will limit the dynamic response. The effects of the other frequencies are usually small and will decay rapidly enough to be considered negligible. Therefore, only the first or fundamental vibration mode is considered in our analysis. Using Rayleigh's approximate method, the fundamental undamped

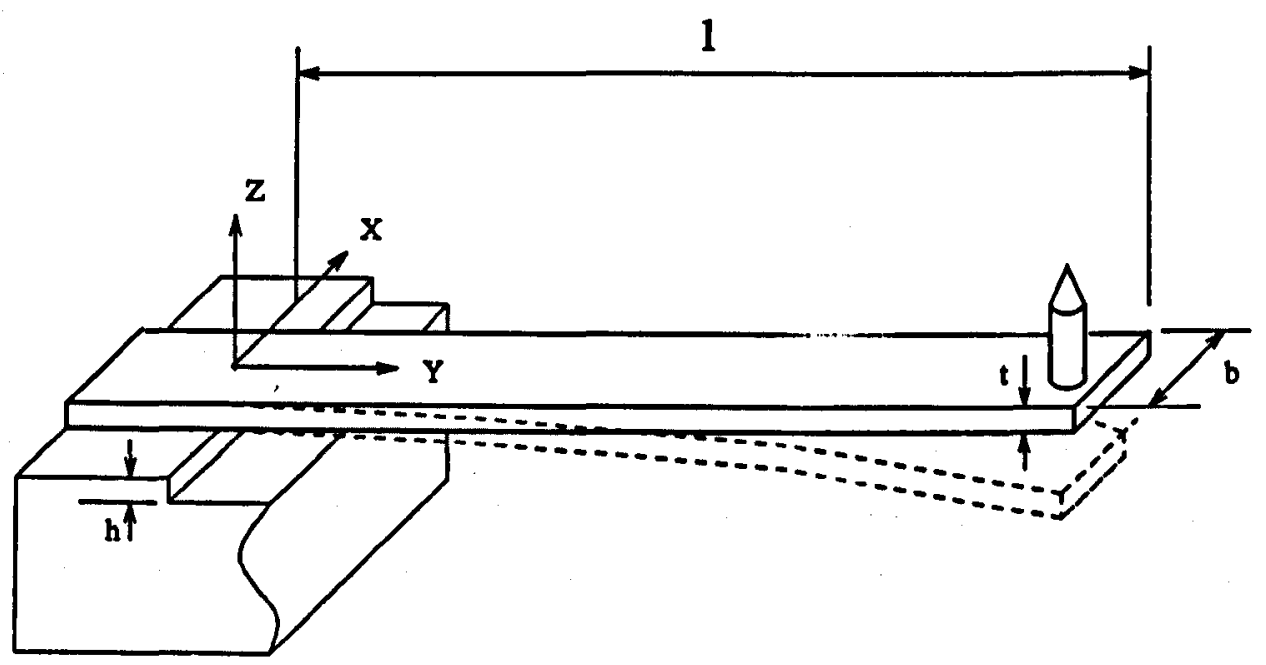

Figure 3.4: Modeling parameters of the force probe

resonant frequency $\omega_{n}$ of a prismatic cantilever with both distributed and concentrated mass is given by

$$
\omega_{n}=\sqrt{\frac{k}{m_{e}}}
$$

where $k$ is the static bending stiffness of the beam and $m_{e}$ is its effective mass both of which are given by $[7,8]$

$$
\begin{aligned}
k & =3 E I / l^{3} \\
m_{e} & =m_{t}+0.24 m_{d}
\end{aligned}
$$

where $m_{t}$ and $m_{d}$ are the concentrated mass (tip mass) and distributed mass (cantilever mass) respectively, $I$ is the second moment of area $\left(=b d^{3} / 12\right.$ for a 
rectangular cross section, where $b$ is the width and $d$ the depth of the beam) and $E$ is its elastic modulus. The probe is modeled as a linear second-order system described by the equation

$$
m_{e} \ddot{z}+c \dot{z}+k z=F
$$

where $c$ is the damping factor dominated primarily by squeeze film acting between the cantilever electrodes and $F$ is the contact force applied to the tip.

Ignoring the effect of the tip mass, the classical solution of the $i$ th deflection mode for lateral vibration of a prismatic cantilever is given by

$$
Z_{i}=\frac{Z_{0}}{2}\left[\cosh k_{i} y-\cos k_{i} y-\alpha_{i}\left(\sinh k_{i} y-\sin k_{i} y\right)\right]
$$

where $Z_{0}$ can be considered as the deflection amplitude at free end which is the function of time, $t$. For the fundamental vibration mode $(i=1)$, we have $k_{1} l=1.875$ and $\alpha_{1}=0.7341$. A simplified deflection model using the equation for static deflection of the beam with both distributed and concentrated mass is given by

$$
\begin{aligned}
Z & =\frac{Z_{0}}{D}\left(\frac{m_{d}(l-y)^{4}}{24 l}+\frac{m_{t}(l-y)^{3}}{6}-C(l-y)+D\right) \\
& =Z_{0} f(y) \\
C & =\frac{l^{2}}{2}\left(m_{t}+\frac{m_{d}}{3}\right) \\
D & =C l-\frac{l^{3}}{6}\left(\frac{m_{d}}{4}+m_{t}\right)
\end{aligned}
$$

Because the gap between the electrodes are minimized to obtain high sensitivity there is a high aspect ratio between electrode separation and beam dimension. This results in significant squeeze film forces and therefore introduces energy dissipation when the beam vibrates. In this model, the squeeze film is approximated as an incompressible and adiabatic film with low Reynolds number, and under 
these conditions Reynolds equation reduces to

$$
\frac{\partial^{2} p(x, y)}{\partial x^{2}}+\frac{\partial^{2} p(x, y)}{\partial y^{2}}=\frac{12 \mu}{h^{3}} V(x, y)
$$

where $p$ is the gauge pressure distribution in the gap, $\mu$ is the viscosity (for air, $\mu=1.862 \times 10^{-5} \mathrm{Nsm}^{-2}$ ), $h$ is the stationary air film thickness and $V$ is the instantaneous velocity of relative motion in the $z$ direction. The distribution of damping pressure in the air gap is obtained from a solution to equation (3.9). Because the mode shape is independent of time (although its magnitude is changing) and, for a slender beam, the bending across the width of the beam is negligible so that the velocity in $x$ is constant at constant $y$, the velocity $V(x, y, t)$ can be represented by two separable functions

$$
V(x, y, t)=V_{0}(t) f(y)
$$

where $V_{0}(t)$ is the amplitude of instantaneous velocity at the free end $\left(=\dot{Z}_{0}\right)$ and $f(y)$ the function of deflection mode obtained from equation (3.7) or (3.8). Assuming a solution to equation (3.9) of the general form [9] ,

$$
p(x, y)=\left(\sum_{n=1,3,5}^{\infty} \cos \frac{n \pi}{b} x\right) Y(y)
$$

and substituting equation (3.8), (3.10) and (3.11) into (3.9), the solution for the pressure distribution is given by

$$
p(x, y)=\sum_{n=1,3,5}^{\infty} \cos R_{n} x\left[B_{n} \sinh R_{n} y+C_{n} \cosh R_{n} y+Y^{*}\right]
$$

where

$$
\begin{aligned}
& Y^{m}=A_{1} y^{4}+A_{2} y^{3}+A_{3} y^{2}+A_{4} y+A_{5} \\
& R_{n}=\frac{n \pi}{b}
\end{aligned}
$$




$$
\begin{aligned}
A_{1} & =-\frac{m_{d} Q_{n}}{24 l R_{n}^{2} D} \\
A_{2} & =-\frac{m_{t} Q_{n}}{6 R_{n}^{2} D} \\
A_{3} & =\frac{12 A_{1}}{R_{n}^{2}} \\
A_{4} & =\frac{6 A_{2}+C Q_{n} / D}{R_{n}^{2}} \\
A_{5} & =-\frac{Q_{n}}{R_{n}^{2}} \\
C & =\frac{l^{2}}{2}\left(m_{t}+\frac{m_{d}}{3}\right) \\
D & =C l-\frac{l^{3}}{6}\left(m_{t}+\frac{m_{d}}{4}\right) \\
Q_{n} & =\frac{48 \mu(-1)^{\frac{(n-1)}{2}}}{\pi h^{3} n} V_{0}
\end{aligned}
$$

Correspondingly for the model of equation (3.7)

$$
\begin{aligned}
& J^{\prime *}=A_{1} \cosh k_{1} y+A_{2} \sinh k_{1} y+A_{3} \cos k_{1} y+A_{4} \sin k_{1} y \\
& R_{n}=\frac{n \pi}{b} \\
& A_{1}=\frac{Q_{n}}{k_{1}^{2}-R_{n}^{2}} \\
& A_{2}=-\frac{\alpha_{1} Q_{n}}{k_{1}^{2}-R_{n}^{2}} \\
& A_{3}=\frac{Q_{n}}{k_{1}^{2}+R_{n}^{2}} \\
& A_{4}=-\frac{Q_{n}}{k_{1}^{2}+R_{n}^{2}} \\
& Q_{n}=\frac{24 \mu(-1)^{\frac{(n-1)}{2}}}{\pi h^{3} n} V_{0}
\end{aligned}
$$

To determine the constants $B_{\mathfrak{n}}$ and $C_{\mathfrak{n}}$ in equation (3.12), the boundary conditions must be satisfied. At the fixed end of the beam air can not be squeezed out. To set up the boundary conditions satisfying this situation, a double length beam with a symmetrical vibration mode with a stationary node at the centre is assumed, Fig. 3.5, and only half of the resultant pressure distribution is used 
to compute the squeeze film force. In this case, the boundary conditions can be expressed mathematically as

$$
p=0, \quad \text { at } \quad x= \pm \frac{b}{2} \text { and } y= \pm l
$$

and the expression of the velocity distribution has to be rewritten as

$$
V=V_{0} f(|y|)
$$

and correspondingly, $Y^{*}$ in equation (3.12) becomes

$$
Y^{*}=A_{1}|y|^{4}+A_{2}|y|^{3}+A_{3}|y|^{2}+A_{4}|y|+A_{5}
$$

Introducing the boundary conditions into the equation (3.12), the constants $B_{n}$ and $C_{n}$ can be determined

$$
\begin{gathered}
B_{n}=\frac{Y^{*}(-l)-Y^{*}(l)}{2 \sinh (R l)} \\
C_{n}=\frac{Y^{*}(l)+Y^{*}(-l)}{2 \cosh (R l)}
\end{gathered}
$$

Fig. 3.6 shows the calculated pressure distribution along the central axis of a cantilever beam with an air film of thickness $15 \mu \mathrm{m}$. This pressure can be integrated over the $y$ axis to give the total damping force acting on the beam, $F_{d}$

$$
\begin{array}{r}
F_{d}=\iint p(x, y) \mathrm{d} x \mathrm{~d} y \\
-\frac{b}{2} \leq x \leq \frac{b}{2}, \quad 0 \leq y \leq l
\end{array}
$$

For modeling purposes the distributed damping force can be translated to a concentrated damping force $F_{e}$ at the same point as the effective mass or probe tip. Using the simple second order model of equation (3.6), the damping factor,c, 


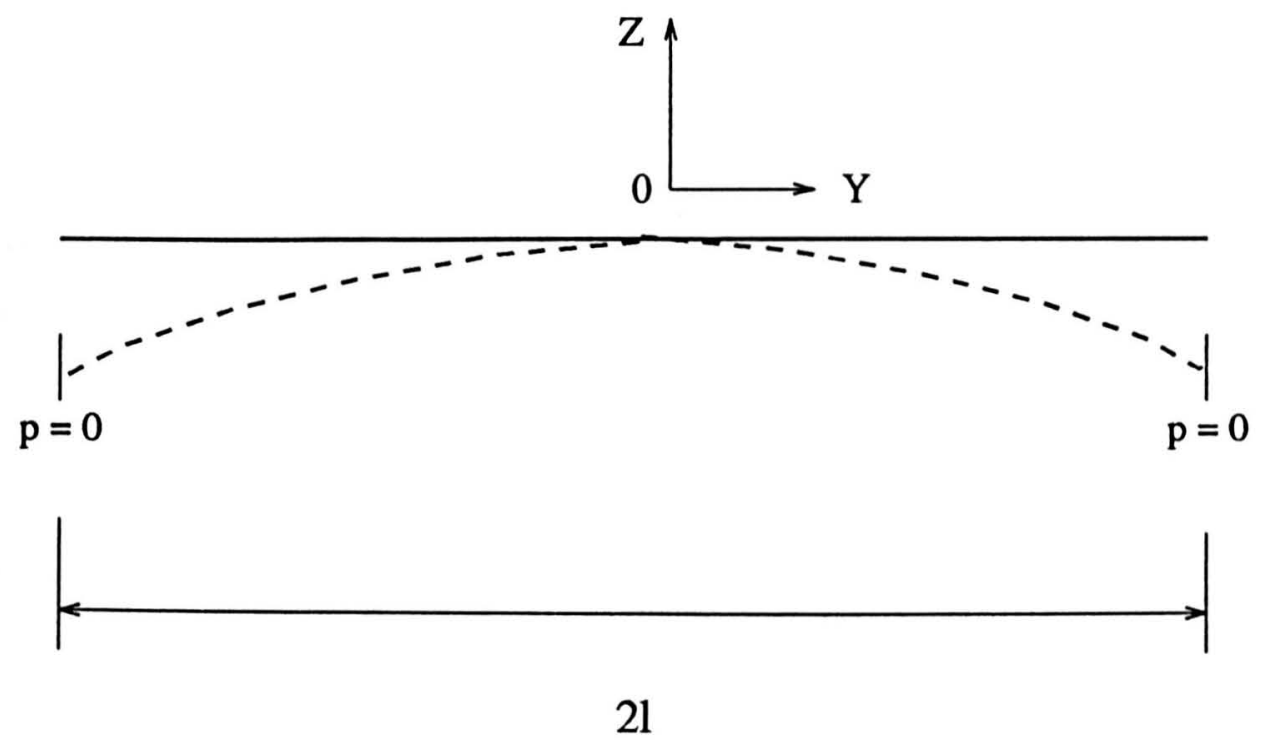

Figure 3.5: Beam model satisfying the boundary condition of zero flow at the clamped end and ambient pressure at the beam edge

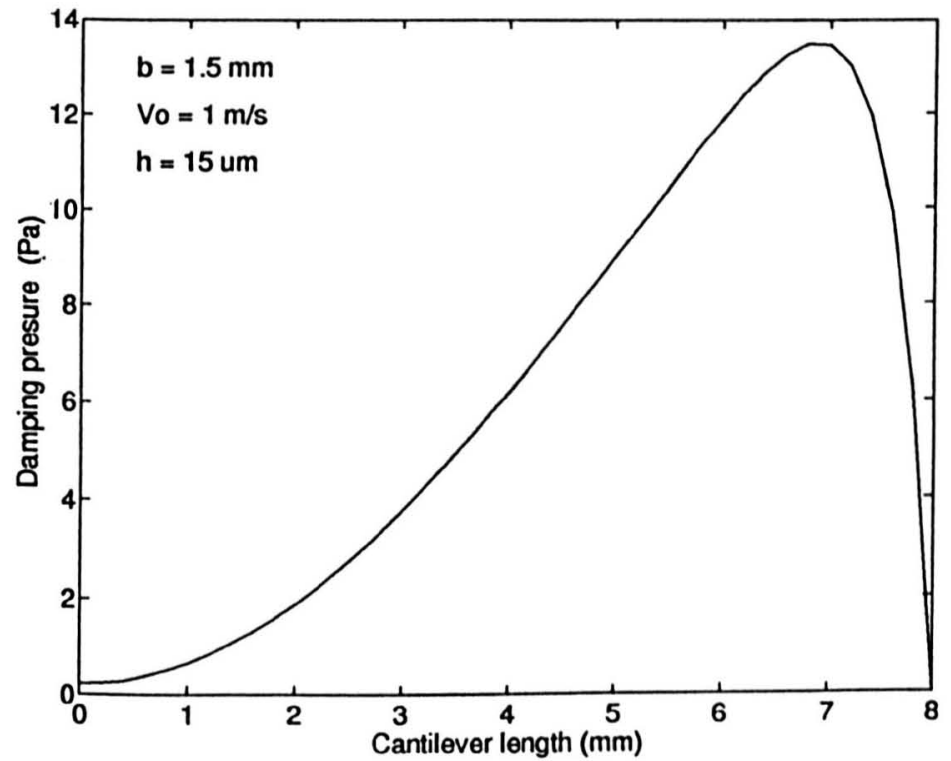

Figure 3.6: The distribution of the damping pressure along central axis of a cantilever beam, $b=1.5 \mathrm{~mm}, V_{0}=1 \mathrm{~mm} \mathrm{~s}^{-1}, h=15 \mu \mathrm{m}$ 
can be derived from the equation

$$
F_{e}=c \dot{z}
$$

where $\dot{z}$ is the velocity at the free end of the beam. Using the principle of virtual work,

$$
F_{e} \Delta_{0}=\iint p(x, y) \Delta(y) \mathrm{d} x \mathrm{~d} y
$$

where $\Delta_{0}$ is an assumed small displacement at the end of the beam, $\Delta(y)$ is deflection profile due to $\Delta_{0}$, given by

$$
\Delta(y)=\Delta_{0} f(y)
$$

Substituting equation (3.19) and (3.21) into equation (3.20), the damping factor is given by

$$
\begin{array}{r}
c=\frac{1}{V_{0}} \iint p(x, y) f(y) \mathrm{d} x \mathrm{~d} y \\
-\frac{b}{2} \leq x \leq \frac{b}{2}, \quad 0 \leq y \leq l
\end{array}
$$

Then the critical damping ratio $\xi$ and damped resonant frequency $\omega_{r}$ can be calculated from the equations

$$
\begin{aligned}
\xi & =\frac{c}{2 \sqrt{k m_{e}}} \\
\omega_{r} & =\omega_{n} \sqrt{1-2 \xi^{2}} \\
f_{r} & =\frac{\omega_{r}}{2 \pi}
\end{aligned}
$$

Fig. 3.7 shows the calculated damping ratios against squeeze film gap for cantilever lengths ranging from 5 to $8 \mathrm{~mm}$. For a wide range of applications, using the deflection model of equation (3.7) with no tip on the cantilever beam, 


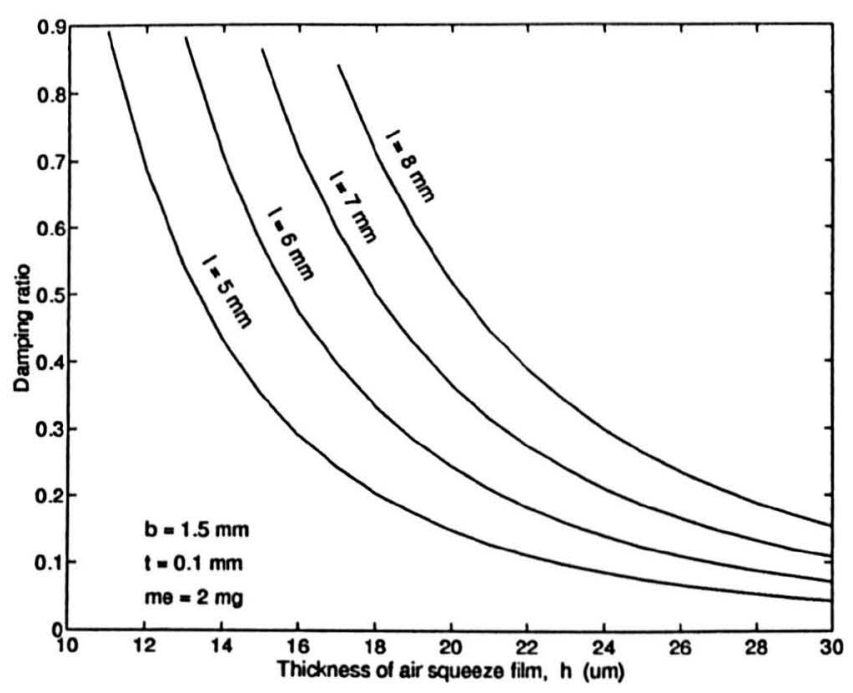

Figure 3.7: The relationship between damping ratio and gap for a variety of lengths of a glass $\left(E=70 G P_{a}\right)$ cantilever with the dimension $\mathrm{b}=1.5 \mathrm{~mm}, \mathrm{t}=$ $0.1 \mathrm{~mm}$ and tip mass $m_{t}=2 \mathrm{mg}$

the damping factors against cantilever beam length for a variety of widths have been calculated. All the calculation are performed on a Sun work station using the software package Mathematica ${ }^{T M}$. The program used for this model solution can be found in the Appendix A of the thesis. The results of the calculation are listed in Table 3.1 and plotted in Fig. $3.8\left(\mathrm{ch}^{3} / \mu\right.$ vs $\left.l \& b\right)$. These curves can be used for beams from 3 to $22 \mathrm{~mm}$ in length and 1 to $3 \mathrm{~mm}$ in width to determine the damping constant for a system of any material, gap medium and thickness of prismatic cantilever. As an example, if one has a silica cantilever beam $\left(E=70 G P_{a}\right.$ of dimension 7 and $1.5 \mathrm{~mm}$ in length and width respectively with an air gap of $15 \mu m\left(\mu=1.862 \times 10^{-5} \mathrm{Nsm}^{-2}\right), c h^{3} / \mu=46.04 \times 10^{-13} \mathrm{~m}^{4}$ can be obtained from figure 3.8, giving a damping factor of $c=46.04 \times 10^{-13} \times$ $1.862 \times 10^{-5} /\left(15 \times 10^{-6}\right)^{3}=0.0254 \mathrm{Nsm}^{-1}$. 


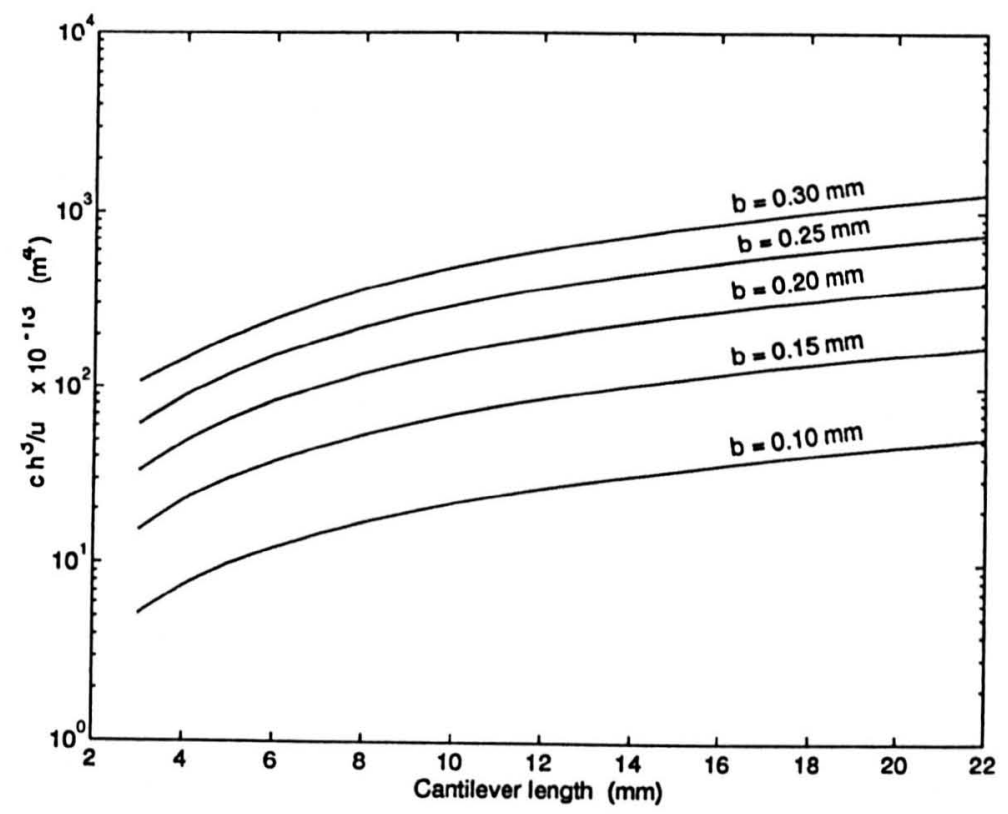

Figure 3.8: The relationship between damping factor and cantilever geometry

\begin{tabular}{|c|c|c|c|c|c|c|c|}
\hline $\mathrm{b}(\mathrm{mm})$ & \multicolumn{7}{|c|}{$\mathrm{l}(\mathrm{mm})$} \\
\hline & 3 & 6 & 9 & 12 & 15 & 18 & 21 \\
\hline 1.0 & 5.20 & 12.31 & 19.66 & 27.08 & 34.53 & 42.0 & 49.47 \\
\hline 1.5 & 15.39 & 38.04 & 62.30 & 87.05 & 112.01 & 137.08 & 162.22 \\
\hline 2.0 & 33.25 & 83.27 & 139.14 & 196.89 & 255.48 & 314.51 & 373.80 \\
\hline 2.5 & 61.78 & 151.56 & 257.00 & 367.66 & 480.70 & 595.02 & 710.11 \\
\hline 3.0 & 107.04 & 246.32 & 421.58 & 608.62 & 801.19 & 996.78 & 1194.20 \\
\hline
\end{tabular}

Table 3.1: The calculated values of $c h^{3} / \mu$ vs $l \& b$ 


\subsection{Experimental assessment of the theoretical model}

The Fig. 3.9 is a schematic diagram of the apparatus used to measure the dynamic response of a variety of force probes. With this the force probe is fixed on a Bruel \& Kjaer (3443) accelerometer, which is, in turn, mounted on a Ling Dynamic (V201) vibrator. A swept-sine signal from an Advantest (TR98021) signal generator was fed to the vibrator through a Derritron (25WT) power amplifier. The signal response from accelerometer and force probe is then recorded using an Advantest (TR9403) digital spectrum analyser and the frequency response computed. Some typical measurement results are shown in Fig 3.10 to 3.12. Fig. 3.10 is the transfer function of an underdamped force probe (No.13 in Table (3.2)). From this, a resonant frequency of $1625 \mathrm{~Hz}$ is observed along with a rapid $180^{\circ}$ phase change, typical of a second-order dynamic response. Fig. 3.11 shows the coherence of the same probe, indicating good linearity between input and output over all frequencies below $3 \mathrm{kHz}$ and thus justifying the assumption of small oscillations and therefore linear frequency response. Fig. 3.12 is the transfer function of a overdamped force probe $\mathrm{No.3}$, in which no resonant frequency can be seen.

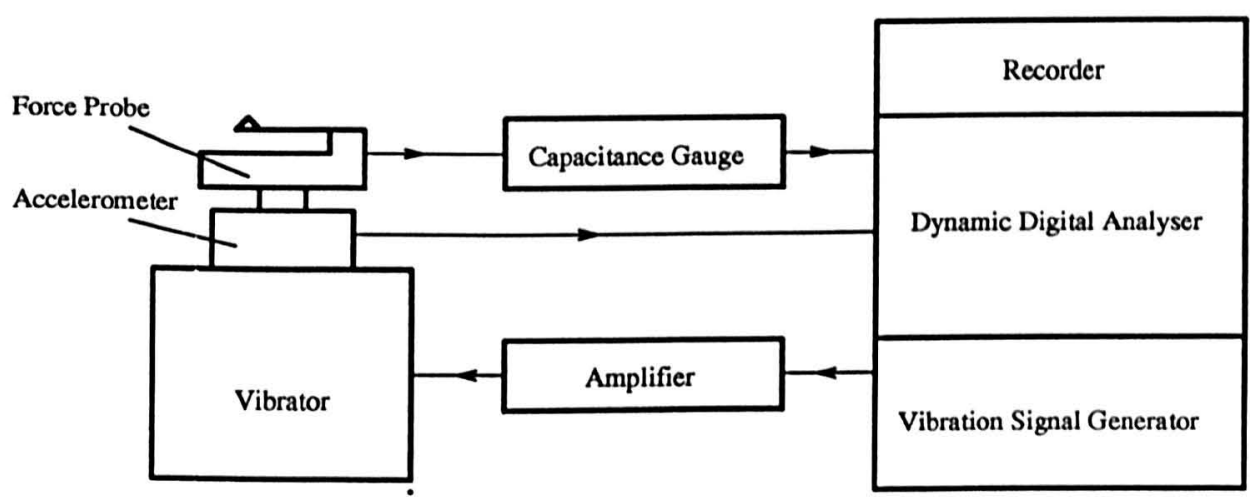

Figure 3.9: The schematical diagram of the dynamic testing system 


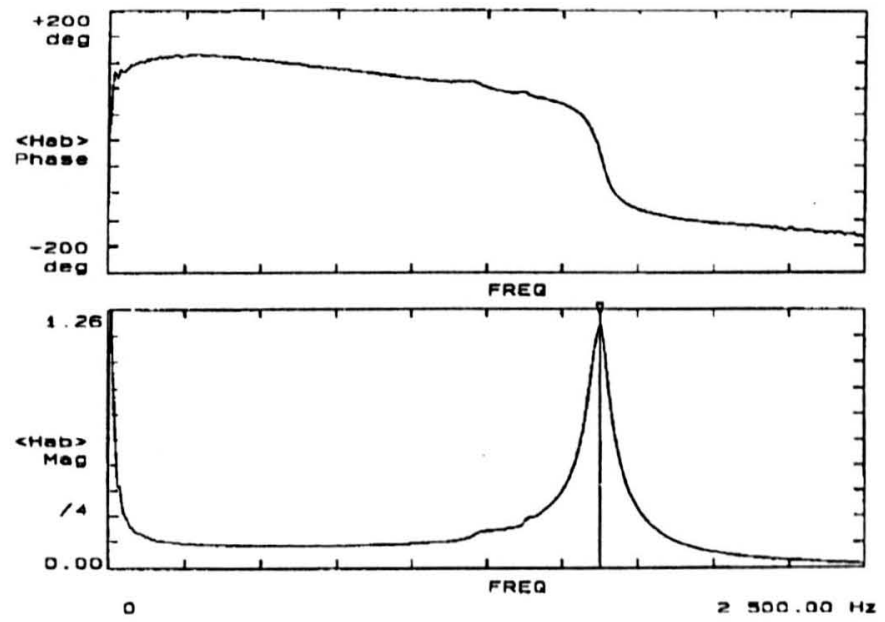

Figure 3.10: The measured transfer function of the force probe

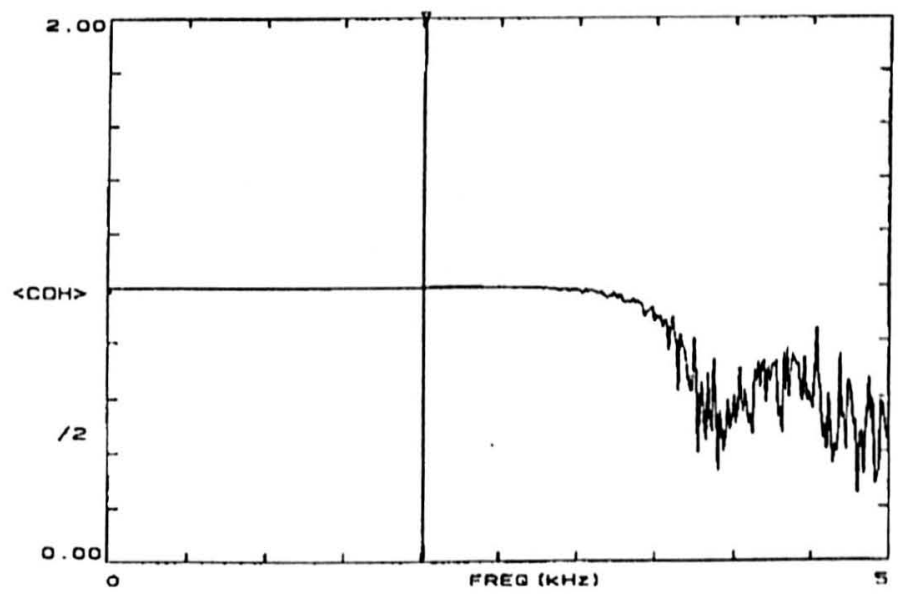

Figure 3.11: The measured coherence function of the force probe 


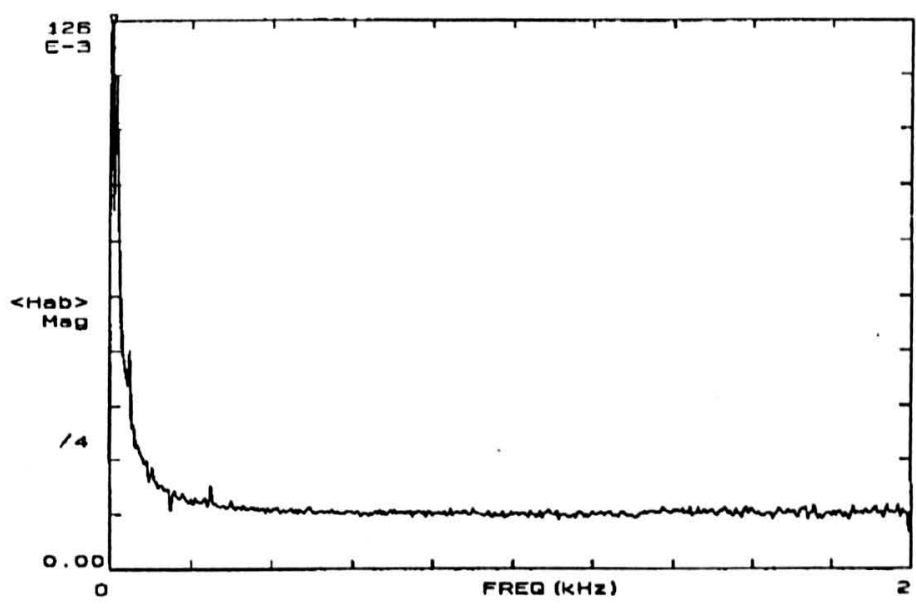

Figure 3.12: The measured transfer function of over damped force probe

More than twenty force probes were produced and their dynamic characteristics measured for comparison with theoretical results. However, some were found to be so overdamped that their damping ratios could not be measured. The remaining results are listed in Table 3.2 and plotted in Fig. 3.13. The parameter $h$ (air gap) is from the measurements using a SIP optical micrometer and the tip mass is measured using precision balance with $0.1 \mathrm{mg}$ resolution. Comparing these results, a. relatively small difference between theoretical and experimental values can be seen. The difference between theoretical and experimental results can be analyzed in two aspects, modeling and experimental errors.

A potential error could be introduced by the simplification of the assumed vibration mode. To assess this, two deflection models, equation (3.8) which is based on the static deflection curve of a prismatic beam with a concentrated mass (tip mass) at one end and equation (3.7) which is the dynamic distortion curve of a simple prismatic beam, were used in the calculations and the results are compared in Fig. 3.14. Fig. 3.15 shows that the profile of equation (3.8) is more curved than that of equation (3.7) due to the effect of tip mass, so the integrated speed along the former beam will be smaller, resulting a lower value 


\begin{tabular}{|c|c|c|c|c|c|c|c|c|c|}
\hline Probe & $\begin{array}{c}t \\
(\mu m)\end{array}$ & $\begin{array}{c}l \\
(m m)\end{array}$ & $\begin{array}{c}h \\
(\mu m)\end{array}$ & $\begin{array}{c}f_{r c} \\
\left(H_{z}\right)\end{array}$ & $\begin{array}{l}f_{r t} \\
\left(H_{z}\right)\end{array}$ & $\xi_{c}^{*}$ & $\xi_{c}$ & $\xi_{t}$ & error \\
\hline No. 1 & 195 & 7.0 & 6.0 & - & - & 4.546 & 4.634 & - & - \\
\hline No. 3 & 105 & 6.0 & 11.2 & - & - & 1.276 & 1.325 & - & - \\
\hline No. 13 & 195 & 8.0 & 24.5 & 1667 & 1625 & 0.094 & 0.095 & 0.044 & 0.050 \\
\hline No. 15 & 195 & 7.0 & 10.2 & 1781 & - & 0.925 & 0.943 & - & - \\
\hline No. 20 & $\overline{105}$ & 6.0 & 18.6 & 1082 & 1130 & 0.279 & 0.289 & 0.325 & 0.046 \\
\hline No. 23 & 195 & 7.5 & 17.7 & 1791 & 1765 & 0.211 & 0.215 & 0.225 & 0.014 \\
\hline No. 26 & 105 & 5.0 & 18.5 & 1530 & 1449 & 0.173 & 0.181 & 0.110 & 0.063 \\
\hline No. 101 & 105 & 5.7 & 18.8 & 1207 & 1190 & 0.235 & 0.245 & 0.257 & 0.022 \\
\hline No. 102 & 105 & 5.2 & 14.0 & 1227 & 1200 & 0.445 & 0.463 & 0.466 & .021 \\
\hline No. 108 & 105 & 6.2 & 26.5 & 1105 & 1100 & 0.105 & 0.109 & 0.148 & $\sqrt{3}$ \\
\hline No. 109 & 1.05 & 6.0 & 22.5 & 1149 & 1150 & 0.157 & 0.163 & 0.180 & 0.023 \\
\hline \multicolumn{10}{|c|}{$\begin{array}{l}t \text { - thickness of the cantilever; } \\
1 \text { - length of the cantilever; } \mathrm{h} \text { - thickness of the air film between the electrodes; } \\
\xi_{c} \text { - calculated damping ratio; } \\
\xi_{t} \text { - testing value of damping ratio; } \\
f_{r c} \text { - calculated value of damped resonant frequency; } \\
f_{r t} \text { - measured value of damped resonant frequency; } \\
* \text { - calculated with deflection model equation (3.8); } \\
\text { error }=\left|\xi_{c}^{*}-\xi_{t}\right| \\
\text { For all the probes: } \\
\quad \text { Glass cantilever } E=70 G P_{a} ; \\
\text { Width of the cantilever b }=1.5 \mathrm{~mm} ; \\
\text { Mass of the tip (concentrated mass) } \mathrm{m}_{c}=2 \mathrm{mg}\end{array}$} \\
\hline
\end{tabular}

Table 3.2: The comparison of the results of experiment and calculation 


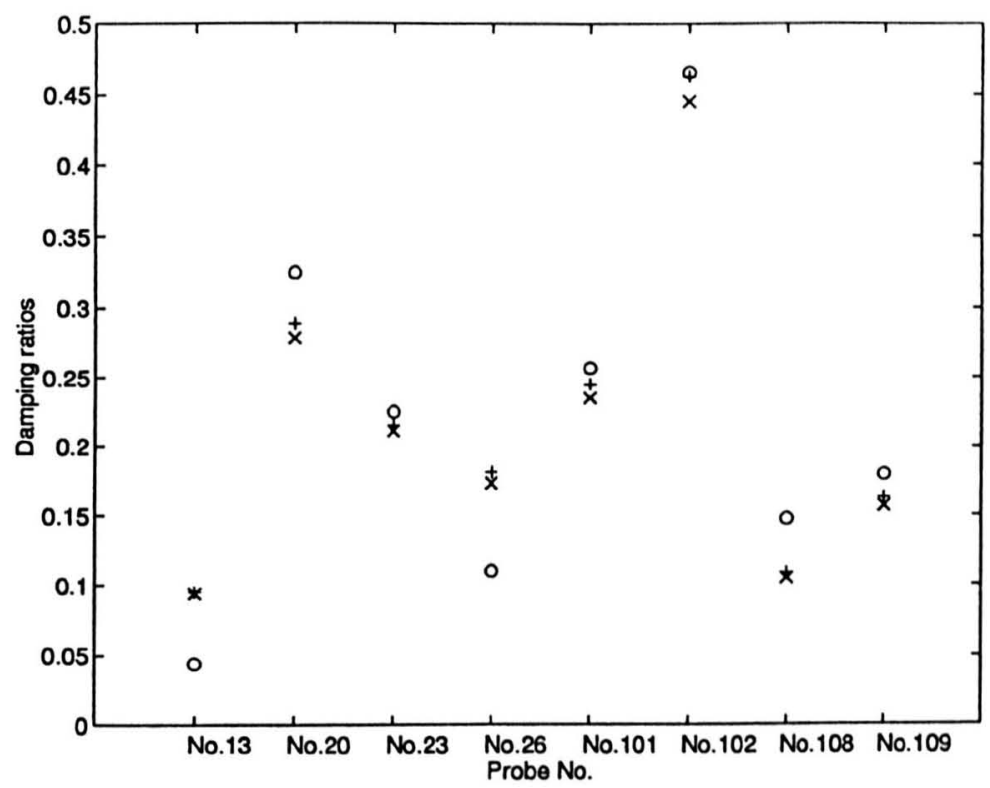

Figure 3.13: The comparison of the results of experiment and calculation. $\mathrm{x}-$ $\xi_{c}^{*},+-\xi_{c}, o--\xi_{t}$

of damping factor c. The shorter the beam, the larger this relative difference. Over the range of geometric dimensions used for these calculations, the maximum difference between calculated results of $c h^{3} / \mu$ is $5.58 \%$ at the point $(l=3 \mathrm{~mm})$, as shown in Fig. 3.14. From this, it can be seen that the damping factors are not very sensitive to the deflection models and the longer the beam, the smaller the error. Generally, if the tip mass is very small compared with the cantilever beam mass, then deflection model of equation (3.7) can be used to calculate the dynamic property of force probe without significant loss of precision, which is also verified by comparison of the calculated results $\xi_{c}^{*}$ and $\xi_{c}$ in Table 2. The main merit of using the model of equation (3.7) is that it is independent of thickness, width, density and elastic modulus of the beam.

For most of the probes in Table 3.2, the measured damping ratios and damped resonant frequency were respectively higher and lower than the values calculated from the mathematic model. A large number of factors will influence the experimental results such as probe dimensions, viscosity of ambient atmosphere, 


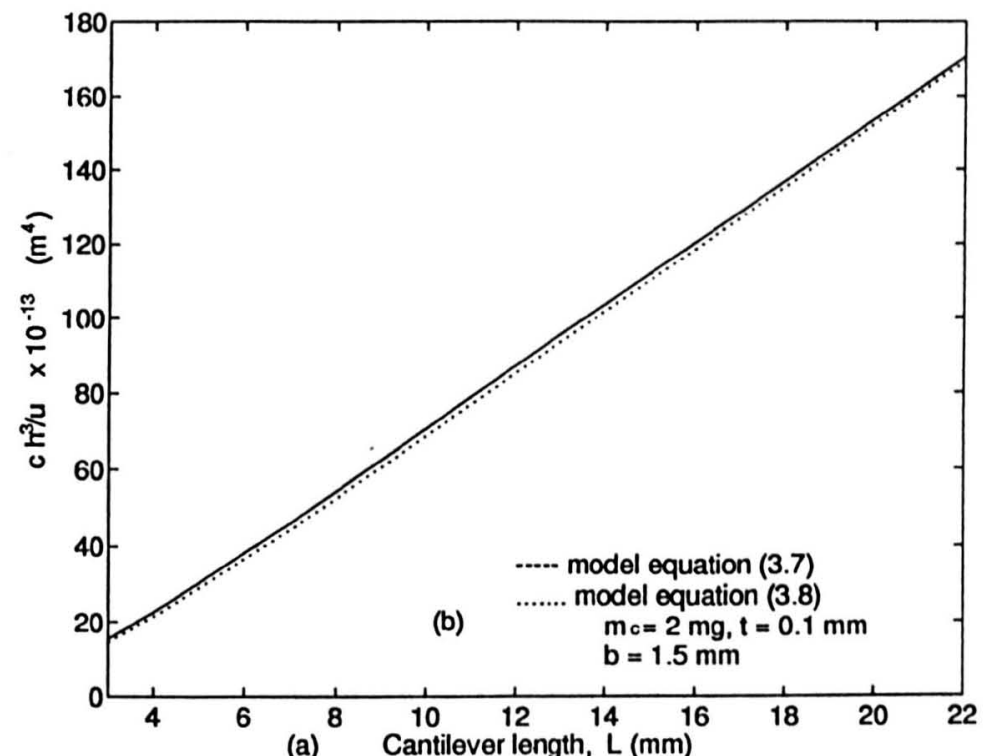

(a) Cantilever length, $L(\mathrm{~mm})$

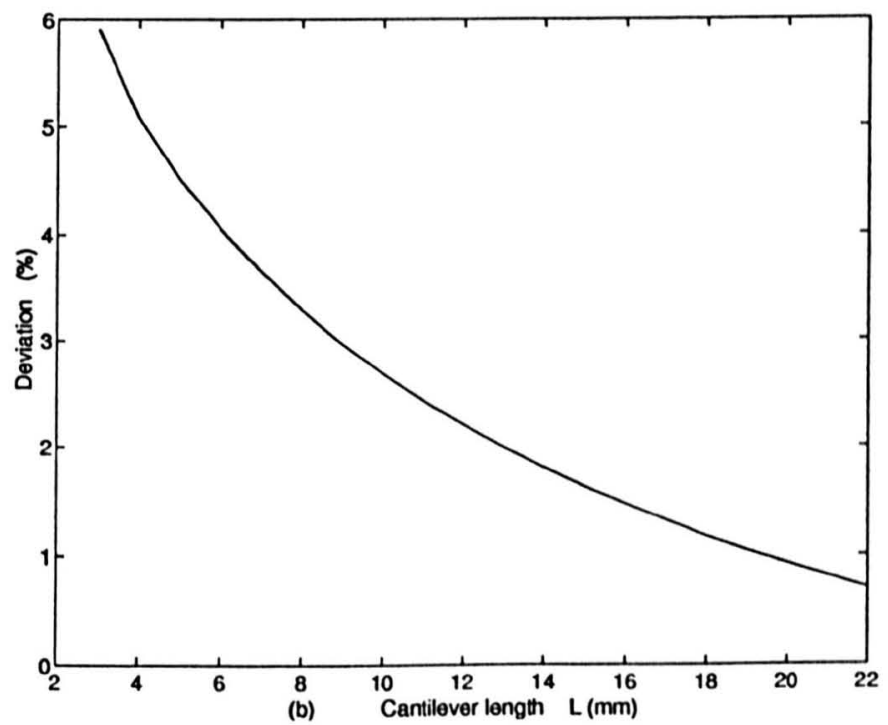

Figure 3.14: Calculated damping forces using different deflection models, $(a)$ results, $(b)$ difference between the two theoretical models 


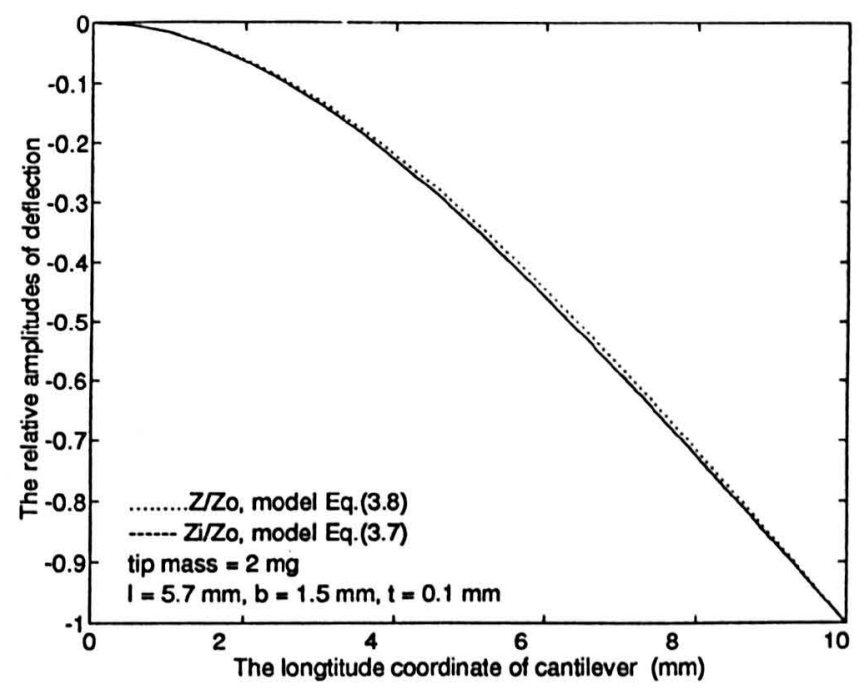

Figure 3.15: The comparison of the profiles of two deflection models

internal losses, and rigidity of the mounting. The sensitivity of the predicted damping ratio to errors in geometric dimensions has been assessed and the results are listed in Table 3.3 in which probe No.101 (in Table 3.2) is taken as an example where all measurement errors are assumed to be $5 \%$ of the measured parameters. From this table it can be seen that damping ratio errors are very sensitive to the thickness of capacitance gap and the beam parameters $b$ and $l$. This illustrates the necessity for stringent manufacturing tolerances if the damping ratio, and therefore dynamic characteristics, of such probes are to be controlled. Due to the small dimensions of our probes, it was very difficult to assess the errors in this experiment. However, we feel that, in general, we achieved measurements of geometric parameters to within a few percent. The separation $h$, being less well defined, is probably subject to an error margin between 5 and $10 \%$. Table 2 shows the error between calculated and measured damping ratios. This shows a standard deviation of 0.0175 with a worst case error of 0.063 . If probes with optimal damping ratio of 0.6 were desired, the present manufacturing process would therefore show a standard deviation of approximately $3 \%$ which will be 
acceptable in view of the wide margin of 0.4 to 0.8 for good measurement fidelity of contact probes.

\begin{tabular}{|c|c|c|c|c|c|}
\hline & $\begin{array}{c}\Delta h= \\
h \times 5 \%\end{array}$ & $\begin{array}{c}\Delta l= \\
l \times 5 \%\end{array}$ & $\begin{array}{c}\Delta b= \\
\mathrm{b} \times 5 \%\end{array}$ & $\begin{array}{c}\Delta t= \\
t \times 5 \%\end{array}$ & $\begin{array}{c}\Delta m_{t}= \\
m_{t} \times 5 \%\end{array}$ \\
\hline$\Delta \xi$ & 0.039 & 0.025 & 0.026 & 0.020 & 0.005 \\
\hline$\frac{\Delta \xi}{\xi} \times 100 \%$ & 16.6 & 10.6 & 11.1 & 8.5 & 2.1 \\
\hline
\end{tabular}

The parameters used in calculation are same as the parameters of probe No.101 in table 1 ,

$h=18.8 \mu m, \mathrm{l}=5.7 \mathrm{~mm}, \mathrm{~b}=1.5 \mathrm{~mm}, t=105 \mu m, m_{t}=2 \mathrm{mg} ; \xi=0.325$

All errors of parameter measurement are assumed to be $5 \%$ of the parameters.

Table 3.3: Damping ratio errors caused by parameter errors

\subsection{Parameter design}

\subsubsection{Introduction}

In principle, it is more complicated to optimize the design of the capacitance force probe than that of others such as the probes monitored by electron tunneling or laser interferorneters. For the capacitance force probe, the parameters of the cantilever beam are not only determined by the stiffness characteristic which is usually the main concern of force probe designers, but also by the capacitance property because the beam's surface opposite to the base block works as one of the capacitance electrodes. The gap thickness is also one of the parameters of importance. The damping effect and sensitivity of the probe are very susceptible to the parameter according to the analysis work introduced above and to be discussed later. In the parameter design of our force probe, four parameters to be determined are: air gap thickness $h$, cantilever beam width $b$, thickness $t$ and length $l$. The objective of optimal design to be achieved is to improve the properties of the probe in the aspects of displacement and force sensitivity, resonant frequency and damping ratio. 


\subsubsection{Mathematical model}

To optimize the properties of the probe, it is, first of all, essential to develop some mathematical models, in which the relationships between the properties and the parameters are revealed.

\section{Capacitance}

If a commercial bridge electronics is chosen for the sensor, there is therefore a requirement on capacitance matching between the probe and the electronic module. We used Queensgate Instruments nanosensor capacitance gauge for which a capacitance of between $2-10 p F$ is required.

Given a static condition with no force on the sensor tip, the relationship between capacitance and probe parameters

$$
C_{0}=\frac{\varepsilon b l}{h_{0}}
$$

where $\varepsilon$ is the dielectric constant, $h_{o}$ is the thickness of the air gap between electrodes and $b$ and $l$ are the width and length of the beam, respectively.

\section{Damping ratio and resonant frequency}

As pointed out at the beginning of this chapter, the dynamic characteristics of the force probe are important for a stylus measurement of high fidelity. A critical damping ratio of between 0.4 and 0.8 corresponds to optimal dynamic conditions. According to the results of dynamic analysis on the force probe introduced before, the damping ratio can be expressed as

$$
\xi=\frac{c}{2 \sqrt{k m_{e}}}=\frac{H(b, l) \mu}{2 \sqrt{k m_{e}} h_{o}^{3}}
$$

where, $k$ and $m_{e}$ are the functions of the parameters of cantilever beam, see equation (3.5), $\mu$ is the viscosity and $h_{o}$ is the stationary air film thickness. $H(b, l)$ is also the function of beam parameters $b$ and $l$. One can obtain $H(b, l)\left(=c h_{o}^{3} / \mu\right)$ 
from Fig. 3.8 by doing polyfit on $c h^{3} / \mu$ with respect to parameters $l$ and $b$, respectively

$$
H(l, b)=(1.71 l-5.09) b^{3}+(2.81 l-0.49) b^{2}-(3.65 l-8.31) b+(1.63 l-5.23)
$$

\section{Deflection and force sensitivity}

As mentioned before, the cantilever beam of the probe will be bent when a small force acted on the tip. The deflection leads to a gap change of capacitance electrodes, resulting a difference in capacitance output. Deflection sensitivity is the ability of the probe to sense small displacement, while the force sensitivity determines the magnitude of the contact force which acts on the surface being measured. Therefore, for the capacitance force probe, to obtain high resolution needs high deflection sensitivity, and to keep low contact force requires high force sensitivity. Considering the state which the tip and surface are in contact, the capacitance of the probe can be expressed as a function of contact force [1]

$$
\begin{aligned}
C(F) & =\frac{\varepsilon b}{a} \tanh ^{-1} \frac{l a}{h_{0}} \\
a & =\sqrt{\frac{h_{0} F}{l^{2} k}}
\end{aligned}
$$

where, $F$ is contact force and $k$ is the stiffness of the beam. Substituting initial static bending $\Delta h_{0}(=F / k)$ into equation (3.28), we have

$$
\begin{aligned}
a & =\sqrt{\frac{h_{0} F}{l^{2} k}}=\frac{1}{l} \sqrt{h_{0} \Delta h_{0}} \\
C\left(\Delta h_{0}\right) & =\frac{l \varepsilon b}{\sqrt{h_{0} \Delta h_{0}}} \tanh ^{-1} \sqrt{\frac{\Delta h_{0}}{h_{0}}}
\end{aligned}
$$


Then, the deflection sensitivity

$$
C_{d}=\frac{d C\left(\Delta h_{0}\right)}{d \Delta h_{0}}=\frac{\varepsilon b l}{2 \Delta h_{0}}\left(\frac{1}{h_{0}-\Delta h_{0}}-\frac{1}{\sqrt{h_{0} \Delta h_{0}}} \tanh ^{-1} \sqrt{\frac{\Delta h_{0}}{h_{0}}}\right)
$$

From which the force sensitivity can be expressed as

$$
C_{f}=\frac{C_{d}}{k}
$$

\subsubsection{Determination of optimal parameters}

With the above mathematical model, it is possible to determine the optimal parameters for the probe. Before doing that, some constraint conditions have to be set on the properties of the probe we concerned, based on the fundamental technical criterion set to the probe.

Firstly, the capacitance of the probe has to be limited between $2-5 p F$ which is required by the commercial bridge electronics.

Secondly, to achieve good fidelity of profiling, the dynamic property of the probe needs to be properly controlled. The results of research work [5][6] indicated that for stylus type of instruments, optimal dynamic conditions correspond to a system critical damping ratio between 0.4 and 0.8 .

Finally, with the concern of the stability and the respond speed of the probe system, the lower limit of the resonant frequency of the probe is set to be 400 $H_{s}$.

Summarizing all these, the constraint conditions for the optimal parameter search are as followings

$$
\begin{gathered}
2<C_{0}<10 \\
0.4<\xi<0.8 \\
400<f_{r}<2000
\end{gathered}
$$


Solving the mathematical model by substituting probe's parameters into equation $(3.24,3.25,3.26,3.30,3.31)$, gives the results in Table $3.4,3.5$ and 3.6, for parametre values which satisfy the constraint conditions. The mass of diamond tip used here is $1.5 \mathrm{mg}$. Other conditions are as same as in Table 3.2. The calculation ranges of the parameters are

$$
\begin{gathered}
0.1(\mathrm{~mm})<b<1.5(\mathrm{~mm}) \\
2.0(\mathrm{~mm})<l<10.0(\mathrm{~mm}) \\
3(\mu \mathrm{m})<h_{0}<10(\mu \mathrm{m})
\end{gathered}
$$

In the results with $h_{0}=3 \mu m$, no group of parameters within the calculation range satisfied the constraint conditions of equation (3.33). So, Table 3.4,3.5 and 3.6 start from $h_{0}=4 \mu m$.

Among the properties, The force sensitivity is usually a main concern of designer. From the equation $(3.30,3.31)$, it can be seen that $h_{0}$ should be chosen to be as small as possible if maximum sensitivity is to be obtained. It is found that reducing $h_{0}$ is mainly limited by the damping ratio constraint. Varying approximately as an inverse cube, the damping ratio is extremely sensitive to the thickness of air gap (see equation (3.26)). This can be improved by modifying other parameters, the width and length of beam, $b$ and $l$. Among them $b$ has dominant effect on damping ratio, and as $b$ reduces so too must the stiffness $k$, which can result in a further increase of the force sensitivity.

Then, the sequence of parameter determination can be derived. First of all, the width of beam, (b), should be chosen as narrow as possible. Then work out the minimum value of air gap $h_{0}$ with which the constraint conditions should be satisfied. Finally, the length of beam $l$ is determined. The same conclusion can be drawn directly from the calculation results in Table 3.4,3.5 and 3.6. 


\begin{tabular}{|c|c|c|c|c|c|c|}
\hline$b(\mathrm{~mm})$ & $l(\mathrm{~mm})$ & $C_{0}(p F)$ & $\xi$ & $f_{r}\left(H_{z}\right)$ & $C_{d}\left(p F / m \times 10^{5}\right)$ & $C_{k}(p F / N)$ \\
\hline 0.3 & 5.0 & 3.319 & 0.490 & 634 & 2.782 & 5722.5 \\
\hline 0.3 & 4.5 & 2.987 & 0.374 & 898 & 2.504 & 3754.6 \\
\hline 0.4 & 4.0 & 3.54 & 0.547 & 896 & 2.968 & 2343.9 \\
\hline 0.4 & 3.5 & 3.098 & 0.386 & 1454 & 2.597 & 1370.0 \\
\hline 0.5 & 3.5 & 3.872 & 0.649 & 764 & 3.246 & 1374.0 \\
\hline 0.5 & 3.0 & 3.319 & 0.431 & 1935 & 2.782 & 741.6 \\
\hline 0.6 & 3.0 & 3.983 & 0.653 & 1023 & 3.339 & 741.6 \\
\hline
\end{tabular}

$h_{0}=5 \mu m$

\begin{tabular}{|c|c|c|c|c|c|c|}
\hline$b(\mathrm{~mm})$ & $l(m \mathrm{~m})$ & $C_{0}(p F)$ & $\xi$ & $f_{r}\left(H_{z}\right)$ & $C_{d}\left(p F / m \times 10^{5}\right)$ & $C_{k}(p F / N)$ \\
\hline 0.3 & 6.0 & 3.186 & 0.400 & 549 & 2.134 & 7585.0 \\
\hline 0.3 & 5.5 & 2.921 & 0.320 & 677 & 1.956 & 5355.7 \\
\hline 0.4 & 5.0 & 3.540 & 0.499 & 712 & 2.371 & 3658.0 \\
\hline 0.4 & 4.5 & 3.186 & 0.380 & 996 & 2.134 & 2400.0 \\
\hline 0.5 & 4.5 & 3.983 & 0.642 & 548 & 2.618 & 2400.0 \\
\hline 0.5 & 4.0 & 3.540 & 0.472 & 1169 & 2.371 & 1498.3 \\
\hline 0.5 & 3.5 & 3.098 & 0.332 & 1702 & 2.075 & 878.3 \\
\hline 0.6 & 3.5 & 3.717 & 0.505 & 1468 & 2.490 & 878.3 \\
\hline 0.8 & 3.0 & 4.247 & 0.634 & 1340 & 2.846 & 474.1 \\
\hline
\end{tabular}

Table 3.4: The parameters versus properties of the probe $\left(h_{0}=4,5 \mu \mathrm{m}\right)$ 
$h_{0}=6 \mu m$

\begin{tabular}{|c|c|c|c|c|c|c|}
\hline$b(\mathrm{~mm})$ & $l(\mathrm{~mm})$ & $C_{0}(p F)$ & $\xi$ & $f_{r}\left(H_{z}\right)$ & $C_{d}\left(p F / m \times 10^{5}\right)$ & $C_{k}(p F / N)$ \\
\hline 0.4 & 6.0 & 3.540 & 0.460 & 576 & 1.975 & 5263.0 \\
\hline 0.4 & 5.5 & 2.245 & 0.369 & 741 & 1.810 & 3716.2 \\
\hline 0.5 & 5.0 & 3.688 & 0.488 & 805 & 2.057 & 2538.2 \\
\hline 0.5 & 4.5 & 3.319 & 0.372 & 1114 & 1.851 & 1665.3 \\
\hline 0.6 & 4.5 & 3.893 & 0.567 & 849 & 2.221 & 1665.3 \\
\hline 0.6 & 4.0 & 3.540 & 0.614 & 1380 & 1.975 & 1039.7 \\
\hline 0.7 & 3.0 & 4.130 & 0.592 & 1002 & 2.304 & 1039.7 \\
\hline 0.7 & 3.5 & 3.614 & 0.414 & 1824 & 2.016 & 609.4 \\
\hline 0.8 & 3.5 & 4.130 & 0.558 & 1467 & 2.304 & 609.4 \\
\hline 1.0 & 3.0 & 4.425 & 0.597 & 1800 & 2.468 & 329.0 \\
\hline
\end{tabular}

\begin{tabular}{|c|c|c|c|c|c|c|}
\hline$b(\mathrm{~mm})$ & $l(m m)$ & $C_{0}(p F)$ & $\xi$ & $f_{r}\left(H_{z}\right)$ & $C_{d}\left(p F / m \times 10^{5}\right)$ & $C_{k}(p F / N)$ \\
\hline 0.5 & 6.5 & 3.595 & 0.354 & 564 & 1.502 & 4073.7 \\
\hline 0.6 & 6.0 & 3.983 & 0.443 & 624 & 1.660 & 2957.6 \\
\hline 0.6 & 5.5 & 3.651 & 0.354 & 793 & 1.526 & 2088.3 \\
\hline 0.7 & 5.5 & 4.259 & 0.506 & 686 & 1.78 & 2088.3 \\
\hline 0.7 & 5.0 & 3.872 & 0.394 & 945 & 1.618 & 1426.3 \\
\hline 0.8 & 5.0 & 4.425 & 0.535 & 790 & 1.849 & 1426.3 \\
\hline 0.8 & 4.5 & 3.983 & 0.404 & 1167 & 1.664 & 935.8 \\
\hline 0.9 & 4.5 & 4.480 & 0.527 & 999 & 1.872 & 935.8 \\
\hline 0.9 & 4.0 & 3.983 & 0.384 & 1511 & 1.664 & 584.2 \\
\hline 1.0 & 4.5 & 4.978 & 0.667 & 522 & 2.080 & 935.8 \\
\hline 1.0 & 4.0 & 4.425 & 0.485 & 1373 & 1.849 & 584.2 \\
\hline 1.1 & 4.0 & 4.868 & 0.598 & 1051 & 2.034 & 584.2 \\
\hline 1.1 & 3.5 & 4.259 & 0.415 & 1963 & 1.780 & 342.5 \\
\hline 1.2 & 3.5 & 4.464 & 0.500 & 1780 & 1.942 & 342.5 \\
\hline 1.3 & 3.5 & 5.033 & 0.594 & 1418 & 2.103 & 342.5 \\
\hline 1.4 & 3.5 & 5.421 & 0.695 & 504 & 2.265 & 432.5 \\
\hline 1.6 & 3.0 & 5.310 & 0.597 & 1947 & 2.219 & 184.9 \\
\hline
\end{tabular}

Table 3.5: The parameters versus properties of the probe $\left(h_{0}=6,8 \mu \mathrm{m}\right)$ 
$h_{0}=10 \mu m$

\begin{tabular}{|c|c|c|c|c|c|c|}
\hline$b(\mathrm{~mm})$ & $l(m m)$ & $C_{0}(p F)$ & $\xi$ & $f_{\mathrm{r}}\left(H_{z}\right)$ & $C_{d}\left(p F / m \times 10^{5}\right)$ & $C_{k}(p F / N)$ \\
\hline 0.6 & 7.0 & 3.717 & 0.383 & 599 & 1.242 & 3504.7 \\
\hline 0.6 & 6.5 & 3.452 & 0.317 & 716 & 1.153 & 2605.6 \\
\hline 0.7 & 6.5 & 4.027 & 0.454 & 656 & 1.346 & 2605.6 \\
\hline 0.7 & 6.0 & 3.717 & 0.369 & 828 & 1.242 & 1891.8 \\
\hline 0.8 & 6.0 & 4.284 & 0.502 & 724 & 1.419 & 1891.8 \\
\hline 0.8 & 5.5 & 3.894 & 0.400 & 972 & 1.301 & 1335.7 \\
\hline 0.8 & 5.0 & 3.540 & 0.312 & 1229 & 1.183 & 912.5 \\
\hline 0.9 & 5.5 & 4.381 & 0.522 & 835 & 1.464 & 1335.7 \\
\hline 0.9 & 5.0 & 3.983 & 0.406 & 1178 & 1.331 & 912.3 \\
\hline 0.9 & 4.5 & 3.584 & 0.307 & 1530 & 1.198 & 598.6 \\
\hline 1.0 & 5.0 & 4.425 & 0.514 & 1033 & 1.479 & 912.3 \\
\hline 1.0 & 4.5 & 3.983 & 0.388 & 1485 & 1.331 & 598.6 \\
\hline 1.1 & 5.0 & 4.868 & 0.634 & 693 & 1.626 & 912.3 \\
\hline 1.1 & 4.5 & 4.381 & 0.478 & 1362 & 1.464 & 598.6 \\
\hline 1.1 & 4.0 & 3.894 & 0.348 & 1939 & 1.301 & 373.7 \\
\hline 1.2 & 4.5 & 4.779 & 0.578 & 1104 & 1.597 & 598.6 \\
\hline 1.2 & 4.0 & 4.248 & 0.420 & 1859 & 1.419 & 373.7 \\
\hline 1.3 & 4.0 & 4.602 & 0.499 & 1694 & 1.538 & 373.7 \\
\hline 1.4 & 4.0 & 4.956 & 0.583 & 1391 & 1.656 & 373.7 \\
\hline 1.5 & 4.0 & 5.310 & 0.675 & 760 & 1.774 & 373.7 \\
\hline 1.7 & 3.5 & 5.266 & 0.605 & 1706 & 1.706 & 210.0 \\
\hline 1.8 & 3.5 & 5.576 & 0.681 & 917 & 1.863 & 219.0 \\
\hline & & & & & & \\
\hline
\end{tabular}

Table 3.6: The parameters versus properties of the probe $\left(h_{0}=10 \mu m\right)$ 


\subsection{Fabrication of the force probe}

For the force probe, the thermal stability is a main concern for material selection, because the small thermal capacitance may make it responsive to thermal disturbances due to its small size. Therefore a low thermal expansion material, Zerodur $r M[10]$ is used for the base of the force probe. The Zerodur flat of $4 \mathrm{~mm}$ in thickness is first lapped on both sides and then optically polished on one side. After that, it is cut into the base block size using diamond slicing machine. A small plateau of a few micrometer in depth is then chemically etched on the optical flat surface in a solution of HF \& HCL acids. Usually a $4: 1 \mathrm{HF}(48 \%): \mathrm{HCL}(35.4 \%)$ acid solution is used with the temperature of $18-20^{\circ} \mathrm{C}$. Although many masking techniques are possible, for convenience the unetched part of the surface is masked with electrical insulating tape. Fig. 3.16 shows the curve of etching depth against time, from which a constant etching rate of $\approx 0.3 \mu \mathrm{m} / \mathrm{sec}$ is found. Finally the surface is carefully cleaned with standard acetone and coated by deposition with a $50 \mathrm{~nm}$ aluminium film patterned to produce one of the electrodes and to form a connection between electrodes and external wiring, as shown in Fig. 3.17.

The cantilever beam is machined from a thin glass cover slide of thickness 100 $\mu m$. Two methods have been tried to cut the cantilever from the slide. One is to use a high speed air motor spindle which is mounted on the vertical spindle of a CNC milling machine. A glass slide is first fixed to a piece of float glass using soft wax. With a little heating the wax will be melt so that the glass slide can be easily glued on or removed from the float glass, which is, in turn, fixed on the work table of the CNC milling machine. The cantilever is so cut that the diamond pin tool mills narrow grooves with certain pattern on the glass slide, as shown in Fig. 3.18. $0.5 \mathrm{~mm}$ webs are left at the ends of the cantilever to hold it for later processing. The diameter of the cutting tool is $1 \mathrm{~mm}$ and the spindle speed is between 50,000 and 100,000 r/min., providing a cutting speed of 5.2 $\mathrm{m} / \mathrm{s}$. Because of this low cutting speed, the feed rates have to be kept slow to prevent cutting force breaking the workpiece. Approximately 2 to $3 \mathrm{~mm} / \mathrm{min}$ was 


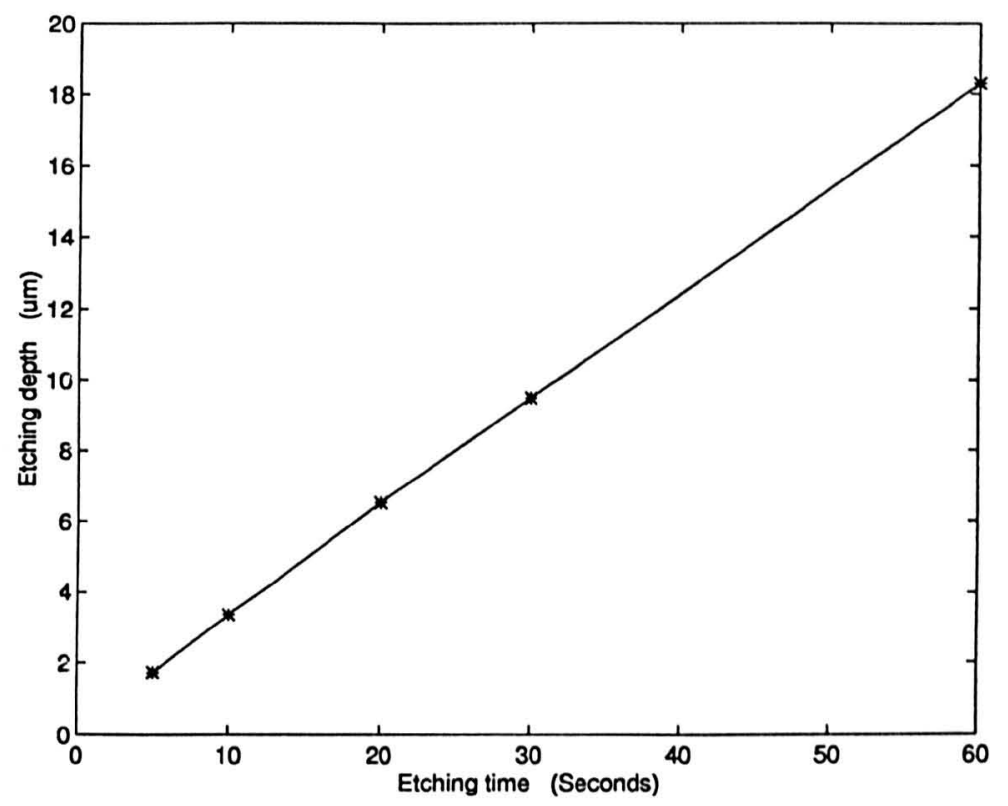

Figure 3.16: The curve of etching depth against etching time

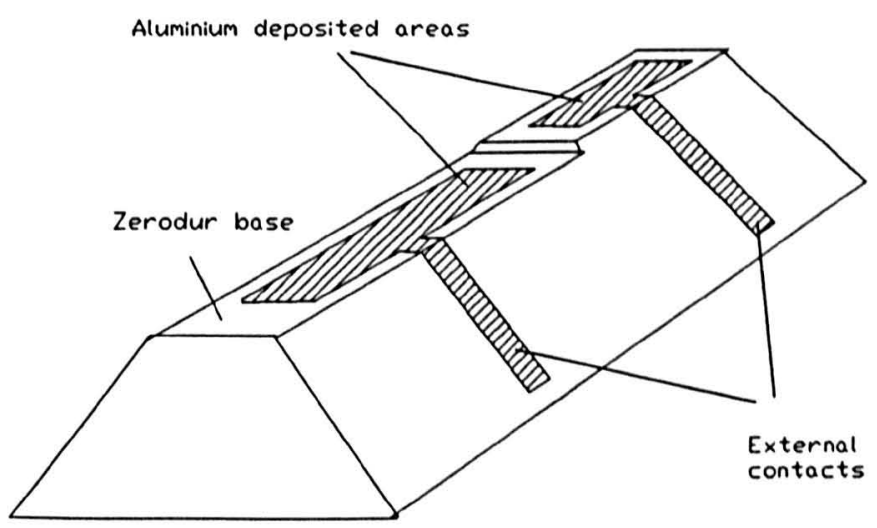

Figure 3.17: The diagram of the pattern of aluminium layer deposited on base block 
used. It is very difficult to increase the cutting speed due to the small diameter of the cutting tool. Low cutting speed generally produces high cutting force which limits the width of the cantilever to be machined down to submillimeter. The advantage of this method is its capability for cutting curved traces so that the components of more complicated shape can be produced. After machining the glass slide is removed from the float glass by heating the wax and cleaned in petrol to remove wax residue. It is then coated with a $50 \mathrm{~nm}$ aluminium by vacuum deposition after cleaned with acetone and methanol.

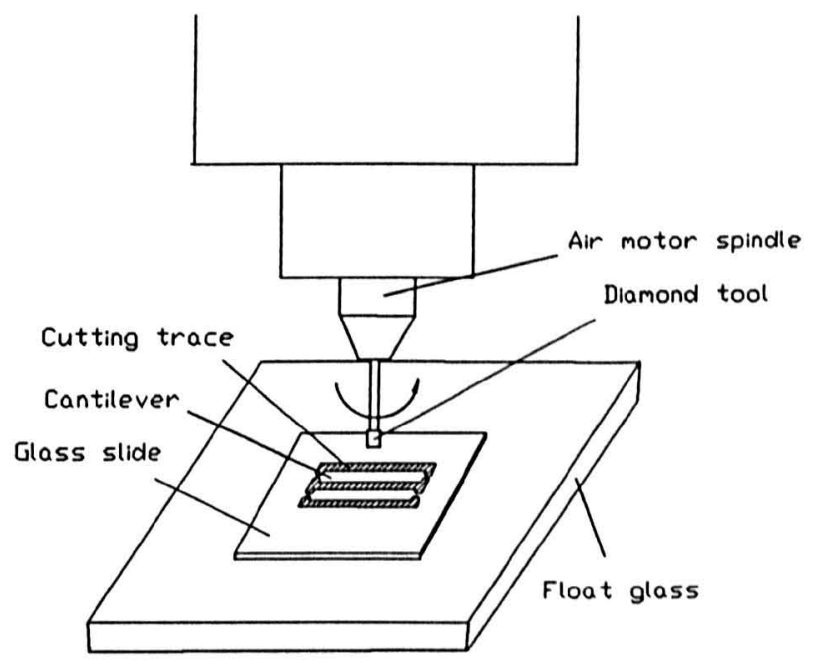

Figure 3.18: Schematic diagram of cantilever machining using milling machine

The other method is to cut the glass slide directly to narrow strips using a diamond wheel slicing machine. The deposition of aluminium has to be done before the glass slide is machined. In this case, to maintain the integration of the deposited surface becomes very important in the following manufacturing process. The glass slide is then sandwiched between two glass pieces, a thick float glass at the bottom and a glass slide of $0.5 \mathrm{~mm}$ thickness at the top. Both pink soft wax and nail polisher have been tried for bonding the workpieces. Soft 
wax is easy and quick for bonding, but not convenient for removing and cleaning so that damage on the surface is difficult to avoid. On the contrary nail polisher takes long time to dry when squeezed between glass pieces, usually about one day, but is quite easy and quick to be dissolved by acetone, resulting in less damage on the surface. The diamond wheel is $200 \mathrm{~mm}$ in diameter and $1.2 \mathrm{~mm}$ in thickness, with rotating speed of $3000 \mathrm{r} / \mathrm{min}$, giving a cutting speed of 31.4 $\mathrm{m} / \mathrm{s}$. 6.4 to $10 \mathrm{~mm} / \mathrm{min}$ feed rates have been used. According to the general principle of diamond cutting, the feed rates have direct effect on the cutting thickness of single diamond grit on the cutting wheel, therefore the cutting force and cutting quality. The higher the feed rate, the bigger the cutting thickness, which corresponds to a higher cutting force and poorer quality. The microscope observation did not show obvious difference in cutting quality within this feed rate range. However the cutting force did have influence on machining process. The very narrow beams can only be obtained with low feed rate. Cantilevers of $0.3 \mathrm{~mm}$ in width have been obtained using this method.

Mating the cantilever to the probe base is the most critical step in the assembly process. To do this, the base is seated on a clean table with step-etched surface facing upwards. The cantilever is then laid on the base, aligned parallel to and facing electrode surface against the base. A metal wire clamp can be used to press the cantilever to contact against the base tightly at the plateau area. The etched-step on the base separates the cantilever and base with a few micrometer gap, in which two facing electrodes form a capacitance sensor. The small gap has to be kept free of dust during the assembly. Once it is built, foreign matters will not be easy to get into the gap because of its very small thickness. Before bonding the cantilever permanently to the base, the probe is checked with electric meter to make sure that the cantilever has been properly located, conducting at the contact area and insulating between electrodes. Then it can be glued to the base with a small fillet of Araldite ${ }^{T M}$ or Superglue ${ }^{T M}$ along the plateau. The final stage is to bond a stylus to the free end of the cantilever. This is done by holding 
the stylus with tweezers, gently dipping its rear back in glue and attaching it on end to the cantilever.

\subsection{Force probe system}

The force probe is versatile to use. It can be usd in either open loop or closed loop control. In the open loop applications, the outputs from the force probe are directly interpreted as the forces acted on the tip or displacements of it. The closed loop system utilizes the outputs of the force probe as feedback signals to maintain the tip contact force constant, so is usually called constant force probe system.

\subsubsection{Linearity}

When the force probe is used in open loop state, the forces acting on the stylus are transferred into a displacement by the deflection of the cantilever and then sensed by the integral capacitance sensor. The outputs of the sensor directly correspond to the forces or displacements. In this case a good linearity of the force probe is essential for its performance and, therefore, it becomes important to determine the linear range. From the equation (3.29) it is clear that the linearity will be affected only by the thickness of capacitance gap $h_{o}$ and deflection magnitude $\Delta h$, and is independent of the width and the length of the cantilever. Fig. 3.19 is the plot of the function (3.29) showing the relationship between the output of capacitance gauge and the deflection of cantilever. From this it can be seen that in the range of small deflection the linearity is quite good. As the deflection increases the nonlinearity grows.

When the ceflection range is kept constant the linearity error will increase as the thickness of the capacitance gap $h_{o}$ reduces, as shown in Fig. 3.20. This indicates that the linearity range varies as the changes of the capacitance gap. It is found that the linearity error is mainly determined by a relative deflection range 


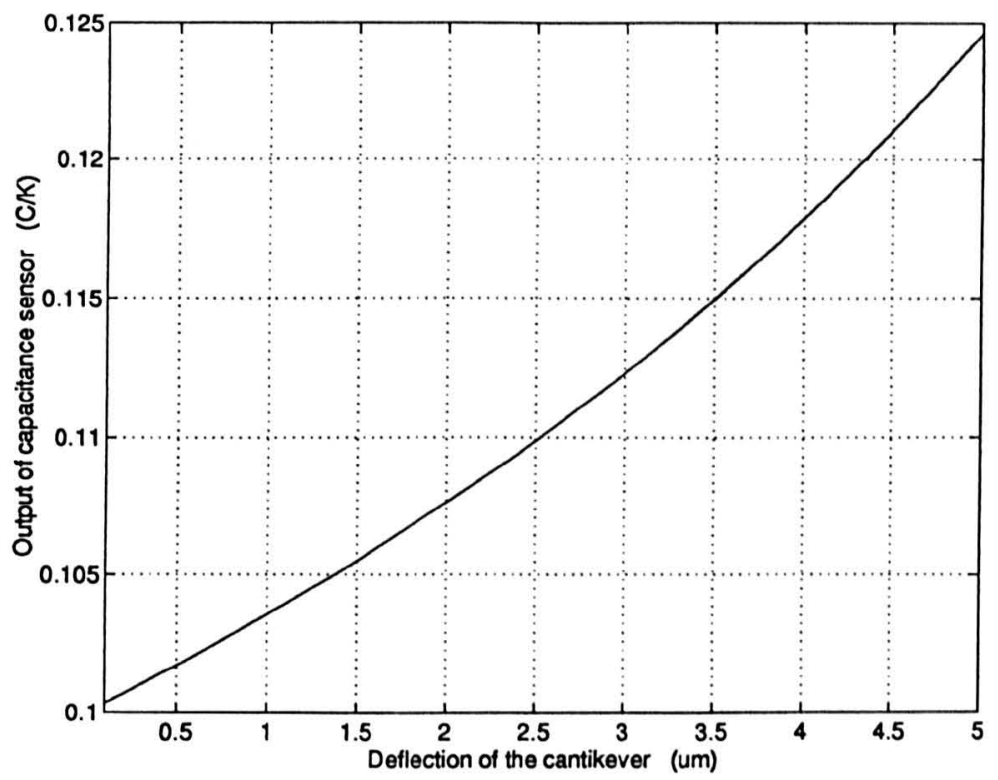

Figure 3.19: Plot of the output of capacitance $C / K(K=l b \varepsilon)$ versus the deflection of cantilever $\Delta h,\left(h_{o}=10 \mu m\right)$, from equation (3.29)

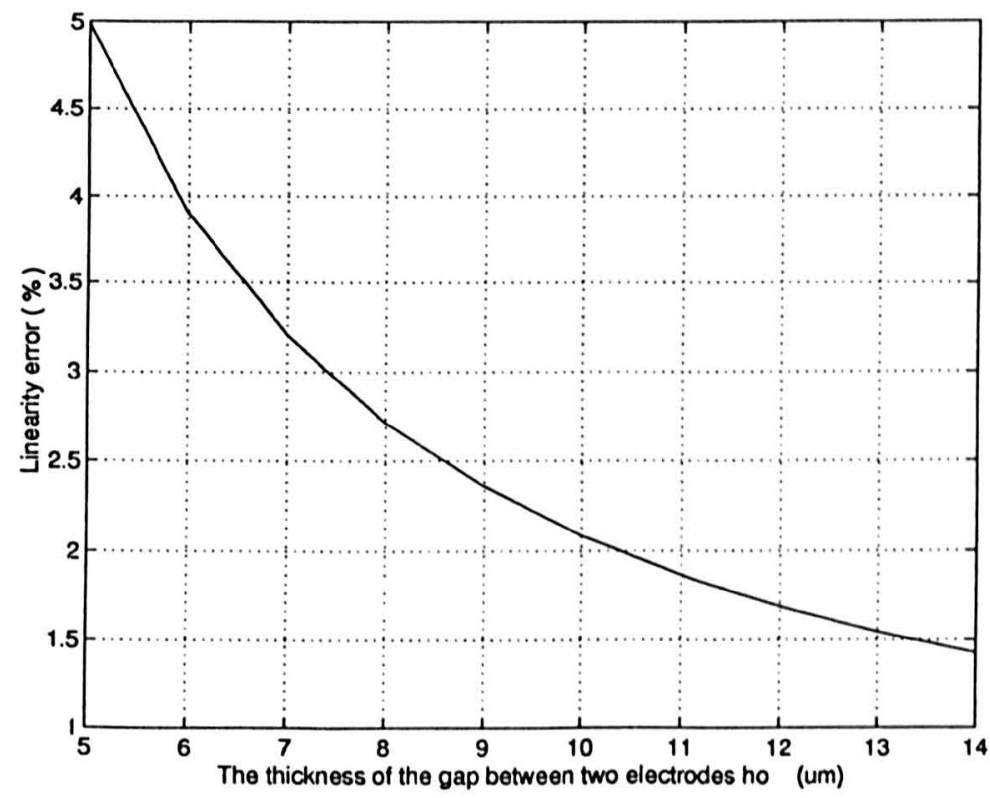

Figure 3.20: Plot of the linearity error against the capacitance gap thickness $h_{o}$ with the same deflection range of $2 \mu \mathrm{m}$ 
$h_{r}$, which is defined as the ratio of absolute deflection range $\Delta h$ and thickness of the gap $h_{o}$, i.e. $h_{r}=\Delta h / h_{o}$. From Fig. 3.21 , it is obvious that there will be no change in linearity if the relative deflection range keeps constant. For example, a relative deflection range of $20 \%$ capacitance gap is calculated to produce a linearity error of $2.08 \%$ in the output of the force probe. If a probe of $10 \mu \mathrm{m}$ capacitance gap is used, a relative deflection range of $20 \%$ corresponds to a 2 $\mu m$ absolute deflection range.

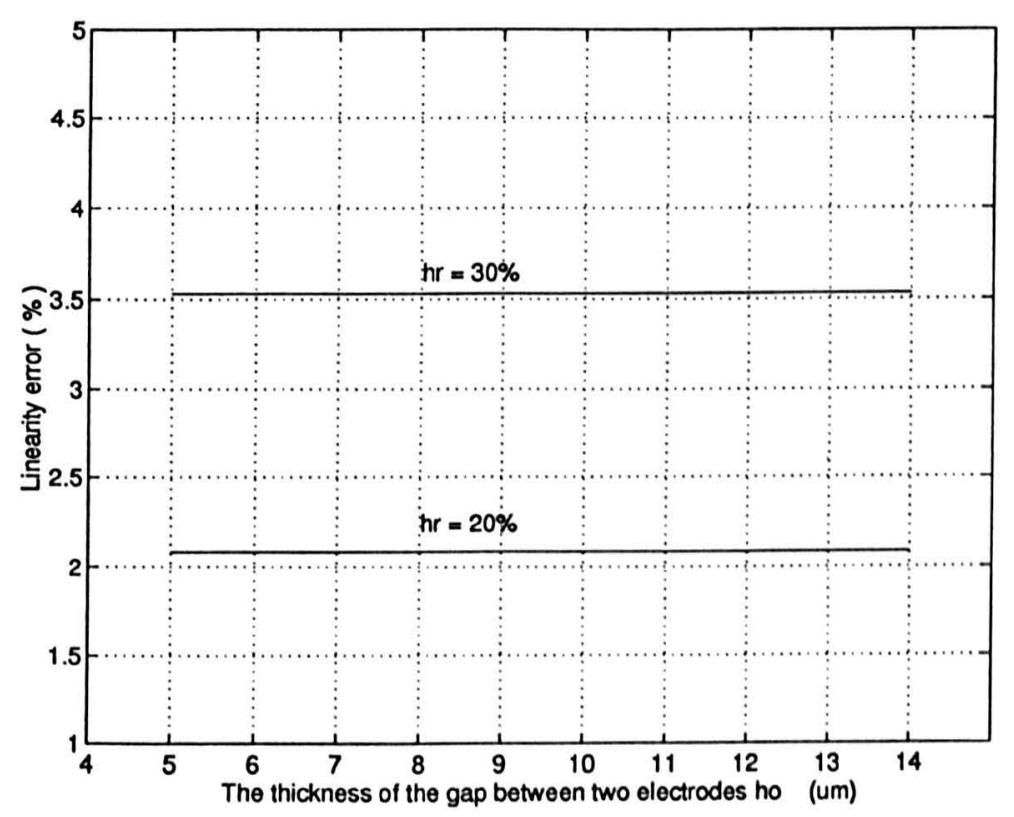

Figure 3.21: Plot of the linearity error against the thickness of capacitance gap $h_{o}$ corresponding to relative deflection ranges of $20 \%$ and $30 \%$

To assess the linearity of the force probe experimentally, a probe is mounted on a linear translator and driven against a surface. The displacement of the translator and the output of the probe are monitored simultaneously. Fig. 3.22 shows a curve of the output of capacitance gauge versus the relative displacement of the probe and surface. The linearities of both calculated and measured are listed in Table 3.7. The experimental results are the averages of several measurements on surfaces of Zerodur, polymer and silicon. 


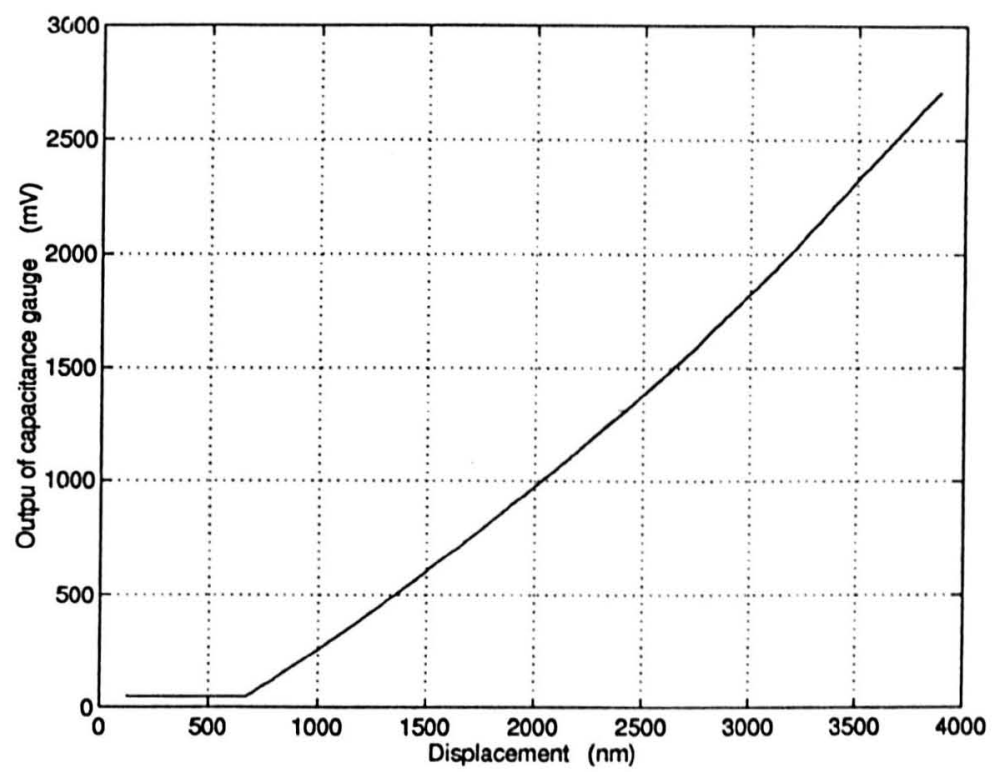

Figure 3.22: The Curve of the output of probe versus the relative displacement between the probe and surface

\begin{tabular}{|c|c|c|}
\hline Deflection range & $\begin{array}{c}\text { Calculated nonlinear } \\
\text { error (\%) }\end{array}$ & $\begin{array}{c}\text { Measured nonlinear } \\
\text { error (\%) }\end{array}$ \\
\hline$h_{o} \times 20 \%$ & 2.08 & 2.29 \\
\hline$h_{o} \times 30 \%$ & 3.52 & 3.74 \\
\hline \multicolumn{2}{|c|}{ Probe: $\quad h_{o}=7.5 \mu \mathrm{m}, b=1.5 \mathrm{~mm}, l=6.5 \mathrm{~mm}$} \\
\hline
\end{tabular}

Table 3.7: The nonlinear errors of the capacitance probe 


\subsubsection{Constant force probe system}

In the application of surface profiling and topographing, a constant contact force between stylus and surface is desired for improving the fidelity of measurement. In this case, a closed loop servo control is usually used, forming a constant force probe system. A constant servo control system usually consists a force probe, a piezo actuator and control unit, as shown in Fig. 3.23. The signals from the capacitance gauge of the force probe are fed back to the controller. The controller then drives the piezo actuator to expand and contract following the change of contact force. The use of piezo actuator is popular in this application. It has a high mechanical response, no backlash and is simple in structure. However, its inherent nonlinearity due to hysteresis and creeping is usually a big problem to the precision profiling and topographing instruments like STM and AFM because the driving signals of the actuator are directly used for interpretation of surface profiles. To overcome the nonlinear problem, a so-called Digital Piezoelectric Translator (DPT) produced by the Queensgate Instruments Ltd. [11] is adopted in our system. An integrated capacitance sensor is built in the translator to monitor the actual expansion of the piezo stacks. The outputs of the sensor can be used for the compensation of the nonlinearity or as the measurement results of the force probe system. Therefore, the nonlinear effect will not be introduced into the measurement. The DPT has a dynamic range of $15 \mu \mathrm{m}$ and a subnanometer resolution. To assess the profiling capability of the constant force probe system, a force probe profiling system was set up as shown schematically in Fig. 3.24. The profiling system is based on a Rank Taylor Hobson Nanostep (an advanced profiling instrument) utilizing its frame, positioning stage and super-precision slideway. The force probe is held on the DPT which is, in turn, mounted on an approaching stage driven manually by a differential micrometer screw. The adjustment of contact force is implemented by the DPT. The specimen is located on a working table which is mounted on a slideway. When the sample is carried to move along the slideway, the surface profile can be obtained by sampling 


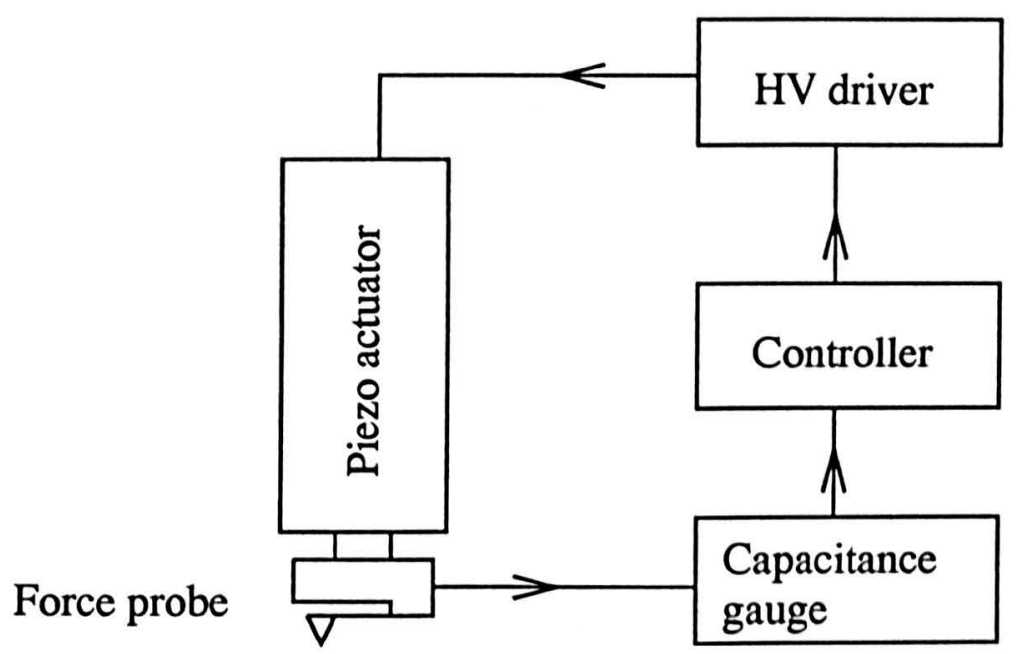

Figure 3.23: Schematic diagram of constant force servo system of the force probe

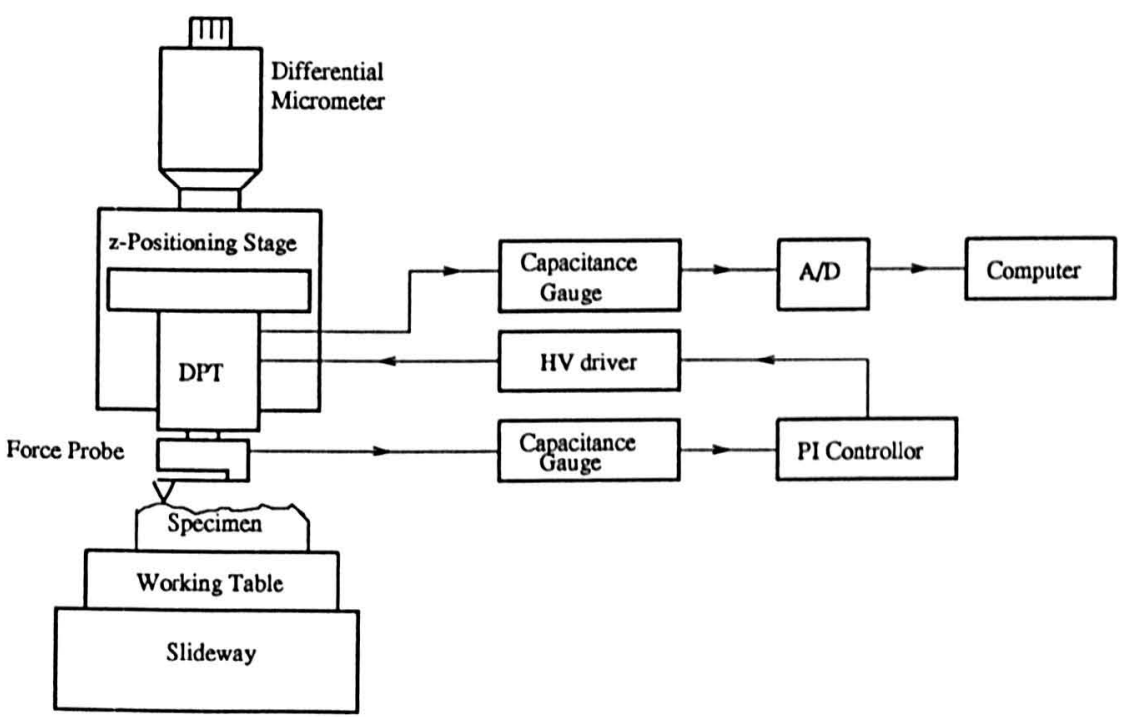

Figure 3.24: The diagram of the constant force profiling system 
the outputs from the capacitance gauge of DPT through a A/D converter to a computer. Alternatively, the specimen may be placed on an $x-y$ stage to obtain the topographic image. This will be discussed later.

Fig. 3.25 shows the profiles of a single diamond turned silicon surface. Fig. 3.25 (a) is the plot of a short range profiling in which the surface features of short wave length are revealed. The section profiles of the diamond cutting trace are shown clearly with an approximate period of $14.3 \mu \mathrm{m}$ and a cutting depth of $35 \mathrm{~nm}$. The repeated feature in the profile can be considered as the projection of the cutting edge shape of the single diamond tool. The long wave length features of the surface are demonstrated in Fig. 3.25 (b). The constant force in the measurement is controlled at about $300 n N$. The detailed assessment of the force probe system will be discussed in Chapter 5 , but from the profiles it is evident that nanometer resolution is achieved. 

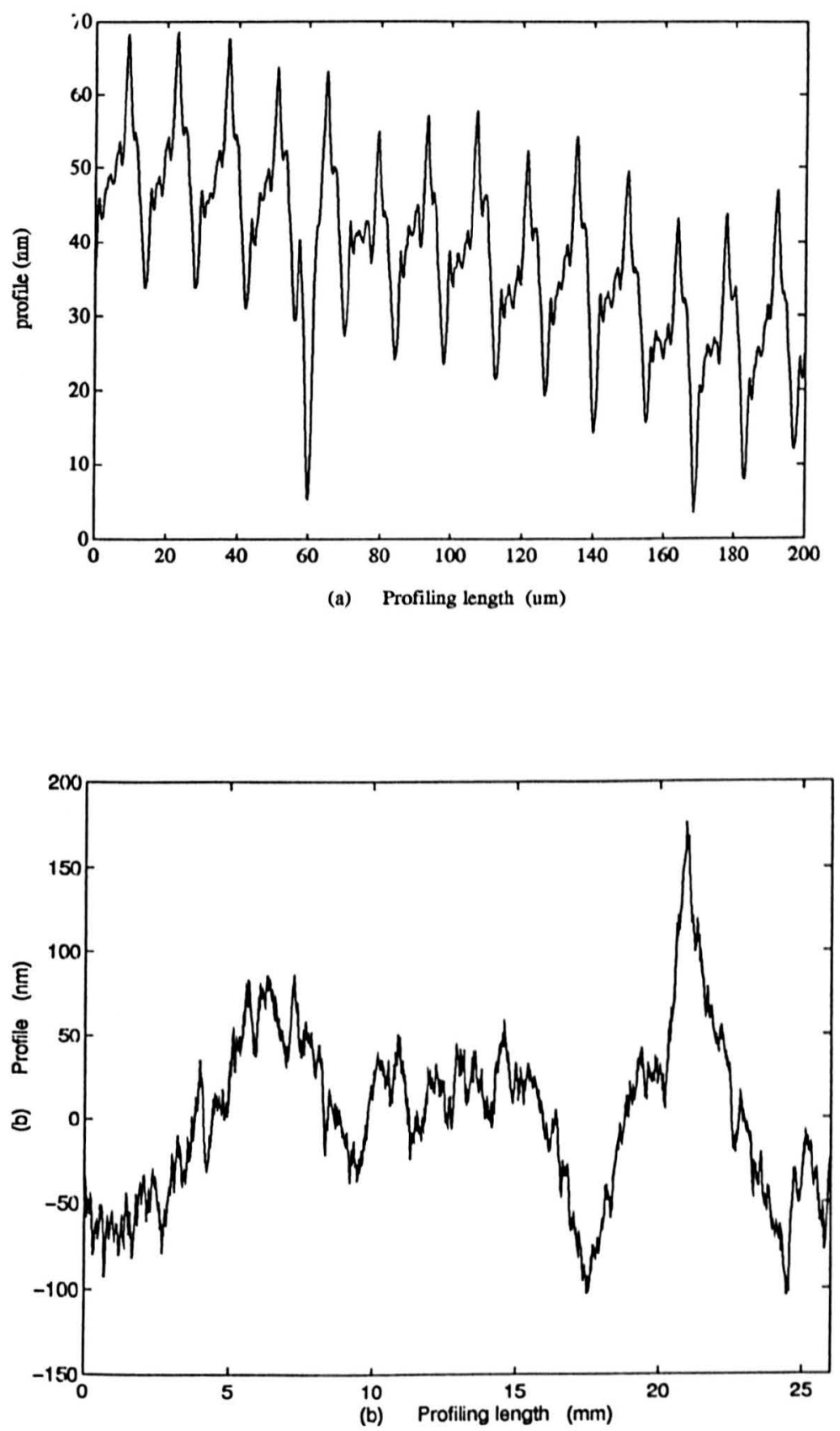

Figure 3.25: Profile of a single diamond turned silicon surface 


\subsection{Conclusion}

The force probe introduced in this chapter demonstrated a very good sensitivity and stability working for surface profiling and topogrphing [2] [12]. The results of the analysis above reveal that the geometric parameters of the force probe have great effects on its performance. When the desired ranges of capacitance, damping ratio and natural frequency are chosen, the parameters of the probe can be optimized to improve its properties in both deflection sensitivity and force sensitivity simultaneously. The former determines probe's capability in resolution, and the later limits the contact force between tip and surface. From the analysis, it is found that there exists a proper sequence for parameter selection. The width of cantilever beam should be first chosen as narrow as possible. Then work out the minimum depth of air gap between two electrodes (mainly limited by damping force). Finally, the length of the beam is determined accordingly.

The squeezing air damping is an unique characteristic for capacitance based force probe. Properly chosing the damping ratio can improve its performance in the aspect of measurement fidelity. The mathematical model which has been verified with experiment can be used for parameter design and prediction of dynamic properties. Moreover, the investigations show that the force probe has an intrinsic good linearity in certain range, which makes it available to be used in closed loop and/or open loop controls. Further applications of the probe are under investigation. 


\section{Bibliography}

[1] L.P.Howard and S.T.Smith, 1992, Long range constant force profiling for measurement of engineering surfaces, Rev. Sci. Instrum. 63(10), 4289 - 4295

[2] L.P.Howard and S.T.Smith, 1994, A metrological constant force stylus profiler, Rev. Sci. Instrum. 65(4), 892 - 902

[3] L.P.Howard, 1993, Advances in Ultra-low Contact Force Nanometric Surface Metrology, $\mathrm{Ph} . \mathrm{D}$ thesis, University of Warwick.

[4] D.J.Whitenouse, 1988, A revised philosophy of surface measuring systems, Proc. Instn. Mech. Engrs., 202 (C3), $169-185$

[5] D.J.Whitehouse, 1990, Dynamic aspects of scanning surface instruments and microscopes, Nanotechnology, 1, 93 - 102

[6] X.Liu, D.G.Chetwynd and S.T.Smith, 1993, Improvement on the fidelity of stylus measurement by active damping control, Meas. Sci. Technol. 4, 1930 $-1340$

[7] J.C.Maltbeak, 1961, The influence of a concentrated mass on the free vibrations of a uniform beam, Int. J. Mech. Sci., 3, 197 - 218

[8] J.P.Den Hartog, 1965, Mechanical Vibrations, 4th ed., McGraw-Hill Book Company, Inc., New York, $429-\not 31$

[9] O.Pinkus and B.Sternlicht, 1961, Theory of Hydrodynamic Lubrication, McGraw-Hill Book Company, Inc. New York. Chapter 7 
[10] Zerodur is a registered trade name of Schott Glass Technologies Inc., 400 York Ave., Durea, PA 18642, USA.

[11] Digital Piezoelectric Actuator is a commercial product of the Queensgate Instruments Ltd, Silwood Park, Ascot, Berkshire SL5 7PW, UK

[12] Y. Xu, S.T. Smith, P.D. Atherton, T. Judge and R. Jones, 1994, A metrological scanning force microscope, Proceedings of ASPE 9th annual meeting, Cincinnati, $23-28$ 


\section{Chapter 4}

\section{Precision X-Y stage}

\subsection{Introduction}

Recently, as the result of rapid development in precision engineering there has been a large increase in the need for precision positioning and scanning systems capable of nar:ometer or, sometimes, even subnanometer level motions. This trend is expected to grow, requiring new design concepts for the exploration of new devices. The major applications of these devices are in the areas of scanning probe microscopy, lithography and micro-fabrication technologies (mainly in the semiconductor industry). Among these, scanning probe microscopy is the most active and rapidly developed technique which provides the main motivation to build new $x-y$ scanning stages of ultra high accuracy, resolution and dynamic characteristics. As reviewed in Chapter 2, the new generation of scanning probe microscopes uses an $x-y$ scanning stage for the implementation of area scanning instead of the conventional tube scanner, due to the requirements of long scanning range and metrological capability.

Because of ihe exceptionally high vertical resolution of the probes such as STM and AFM, for metrological evaluation of surface features down to the atomic level the lateral resolution has to be matched by the scanning stages in similar range. To do this, the primary requirement on stage performance is that the stage 
travels smoothly and rectilinearly in the $x$ and $y$ directions, desirably with no undulations, rumble, vibration or other vertical displacement. In other words, the stage motion should map out an ideally flat datum with a precision greater than the vertical resolution expected. Any vertical erroneous motion could be falsely interpreted as surface roughness or waviness during surface measurement. Since the stage is usually scanned in a closely spaced raster pattern and a high scanning speed is needed to reduce the effect of environment, it is necessary that the stage have sufficient horizontal rigidity and compact size for a high resonant frequency. Scan-to-scan reproducibility so that an accurate measurement of surface feature positions can be made both perpendicular and parallel to the scan traces is also very important to this application.

Crucial to the success of such scanning stages is the availability of actuators and translation mechanisms of very high resolution and precision. The latter is the main concern of this chapter. The performance of the translation mechanism and its general design principles are analyzed and discussed. The design of a precision $x-y$ scanning stage for such applications is described in detail.

\subsection{Flexure translation mechanisms}

Elastic flexure mechanisms have been used for over a century in a wide variety of instruments such as precision balances, tiltmeters, gravitymeters, optical and $\mathrm{x}$-ray interferometers, surface metrology and many other devices which require a smooth and accurate motion over a limited traverse range [1]-[4]. The well known advantages of the flexure mechanisms are:

1) They are friction and wear free. Because there are no sliding pairs between moving parts, no wear will be produced. Therefore, the performance of the mechanisms will keep stable over a complete lifetime.

2) There is no backlash or creep, so that the displacement is smooth and continuous. 
3) The performance is predictable due to the linear relationship between deflections and driving forces over a certain range. The stiffnesses of the flexure members can be completely determined through a knowledge of their geometry and the elastic modulus.

4) They are simple, inexpensive and compact.

Generally, there are two types of spring components used in flexure translation mechanisms, the leaf and notch hinge spring. A basic form of the flexure translation mechanism is a simple rectilinear spring system, as shown in Fig. 4.1. Both of these systems can achieve precise motions. Jones devised a platform supported by a system of parallel horizontal leaf springs which could be moved parallel to itself by applying a force normal to the springs, limiting roll, pitch and yaw to less than 37 arcseconds over a $10 \mathrm{~mm}$ displacement [5]. Scire and Teague developed a single axis notch hinge stage, providing ultrasmooth movements over $75 \mu \mathrm{m}$ with roll, pitch and yaw less than 1 arcsecond [3]. Meanwhile, the performances in nanometer or even subnanometer level have been achieved using these simple flexure translation mechanisms driven by either piezo actuator [3] or a high compliance solenoid/magnet force actuator [6] [7].

\subsubsection{Stiffness}

The stiffness of the flexure springs is one of the most important specifications of concern to the designer. It determines both the static and dynamic characteristics of the system. For the simple leaf spring flexure, there is a pair of cantilevers rigidly attached to both the base and the free moving platform, Fig. 4.1 (a). Each spring then deflects into an $S$-shaped curve, Fig. 4.2. Using elementary beam theory, the stiffness for the leaf spring is [4]

$$
k_{\text {leal }}=\frac{E b d^{3}}{L^{3}}
$$




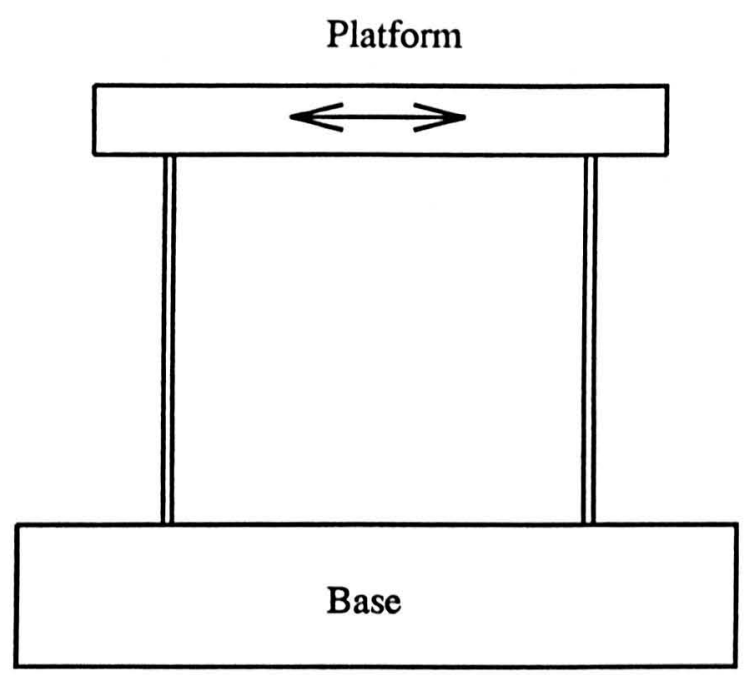

Simple leaf spring rectilinear mechanism

(a)

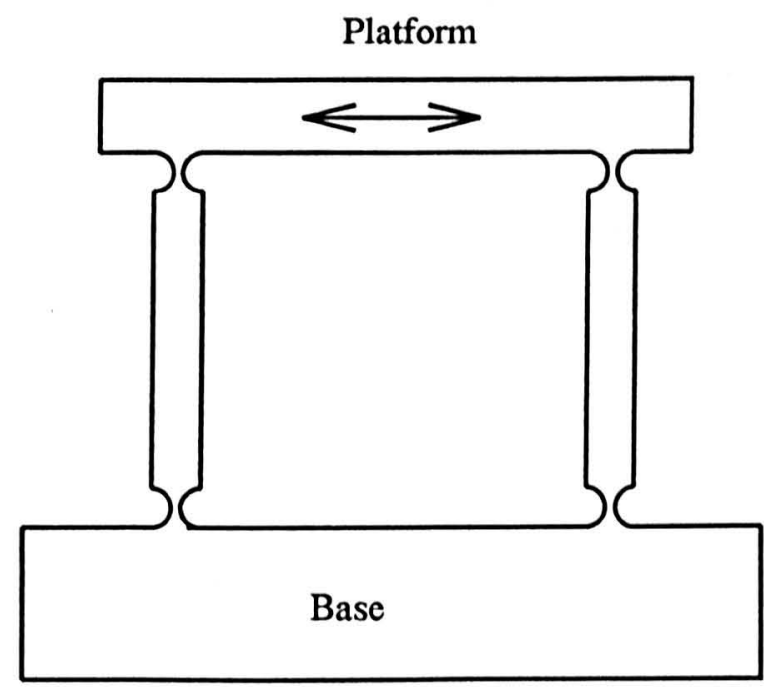

Simple notch hinge rectilinear mechanism

(b)

Figure 4.1: The diagram of simple flexure rectilinear mechanisms 
where $E$ is the elastic modulus, $L$ is the length of the spring, and $b$ and $d$ are width and depth of the cross section of the spring. For notch type rectilinear

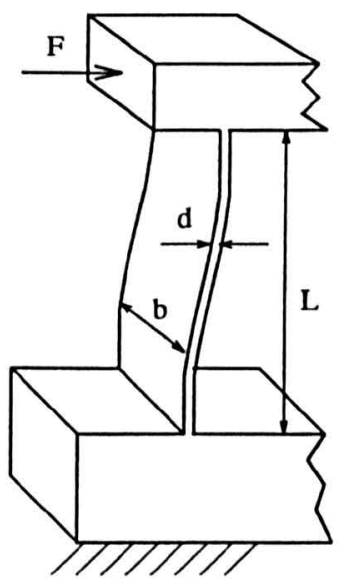

(a)

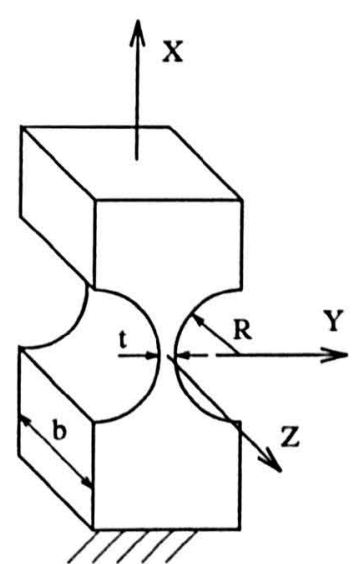

(b)

Figure 4.2: The configurations of the flexure springs

mechanism, the motion of the platform is produced by the elastic compliance of the four flexure notched hinges, Fig. 4.1 (b). The circular shaped hinges are most popularly used in practical applications, mainly because of its ease of manufacture. According to Paros and Weisbord's model [8], the rotation stiffness about the hinge axis ( $z$ axis in Fig. 4.2) can be expressed as

$$
k_{z}=\frac{M_{z}}{\alpha_{z}} \approx \frac{2 E b t^{5 / 2}}{9 \pi R^{1 / 2}}
$$

where $R, t$ and $b$ are the dimensions of the hinge as shown in Fig. 4.2(b). For $t$ $<R<5 t$, an approximation derived empirically from the finite element method gives [4]

$$
k_{z} \approx \frac{E I_{z z}}{2 K R}
$$

where $K=0.565 t / R+0.166, I_{z z}=\frac{1}{12} h t^{3}$

For analysis of system performance, the stiffnesses in other directions are also of interest. Deflections in these axes deteriorate the system's performance 
by reducing accuracy and providing mechanisms for 'lost' motion (as will be discussed later). The stiffnesses of hinge spring along the $x$ and $y$ axes are given by $[8]$

$$
\begin{gathered}
k_{x}=\frac{F_{x}}{\Delta x} \approx \frac{E b}{\pi(R / t)^{1 / 2}-2.57} \\
k_{y}=\frac{F_{y}}{\Delta y} \approx \frac{2 G b}{9 \pi}\left(\frac{t}{R}\right)^{5 / 2}
\end{gathered}
$$

where $G$ is shearing elastic modulus which can be derived from $E: G=E / 2(1+\nu)$, where $\nu$ is Poison ratio. The torsional stiffness of a leaf spring about the $y$ axis can be expressed by

$$
k_{t}=\frac{G J_{b}}{L}=\frac{G d b^{3}}{12 L^{3}}
$$

\subsubsection{Deflection range}

The deflection range of a simple flexure rectilinear mechanism is limited either by its stiffness, through the maximum force that can be applied to it, or by its maximum elastic strain. For the simple leaf spring mechanism (Fig. 4.1 (a)), the permissible displacement is [4]

$$
q_{\text {lea } f_{\max }}=\frac{\sigma_{\max } L^{2}}{3 E d}
$$

where $\sigma_{\max }$ is a maximum allowable tensile stress which is usually 0.3 to 0.1 of the effective yield stress for metal springs. For simple notch hinge rectilinear mechanism, the maximum moment which is allowable on each hinge is

$$
M_{\max }=\frac{b t^{2}}{6 K_{t}} \sigma_{\max }
$$

where

$$
K_{t} \approx \frac{2.7 t+5.4 R}{8 R+t}+0.325=\frac{2.7+5.4(R / t)}{8(R / t)+1}+0.325
$$

is the stress concentration factor caused by the circular notch shape. Combining equations 4.2 and 4.8 , the maximum allowable rotation of the hinge $\theta_{\max }$ and the 
maximum allowable displacement of the platform $q_{\max }$ are given by

$$
\begin{gathered}
\theta_{\max }=\frac{3 \pi \sigma_{\max }(t / R)^{1 / 2}}{4 E K_{t}}=\frac{3 \pi \sigma_{\max }}{4 E} Q \\
q_{\max }=\frac{3 \pi L \sigma_{\max }}{4 E} Q
\end{gathered}
$$

where $Q=(R / t)^{1 / 2} / K_{t}$ is plotted in Fig. 4.3.

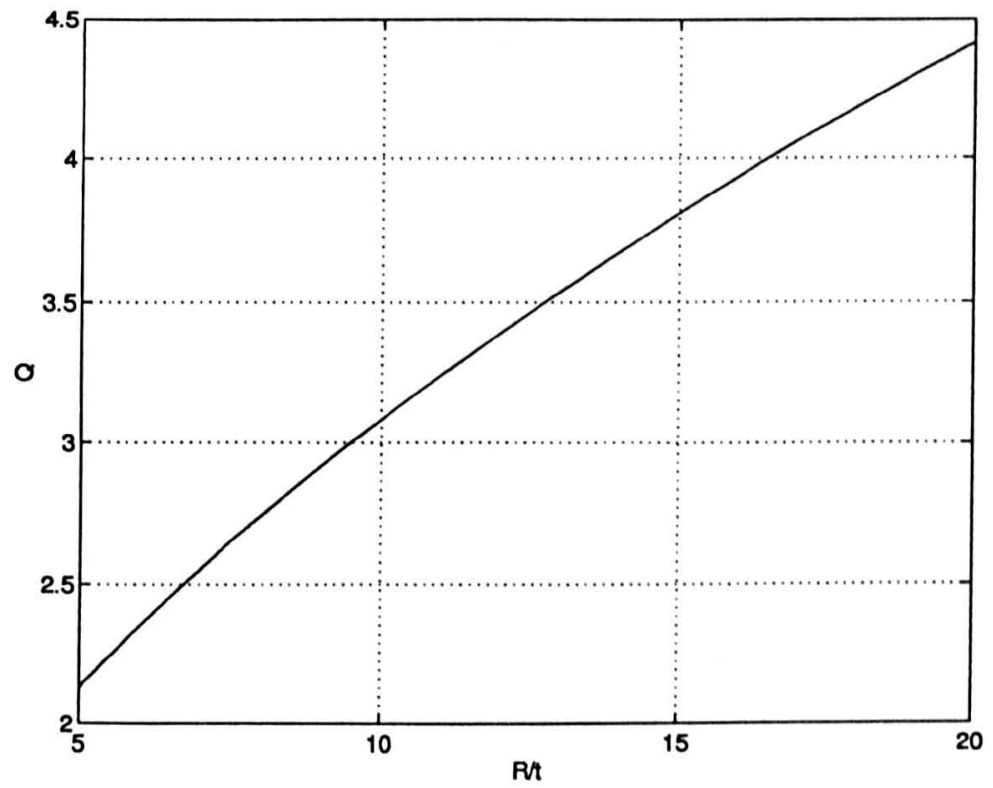

Figure 4.3: Plot of $Q$ in equation (4.10) against $R / t$

From equations (4.1) and (4.7), it can be seen that by keeping $d / L$ constant and increasing $L$ the deflection range of the leaf spring mechanism can be increased without losing system stiffness. However, for notch hinge mechanism of equations (4.2) and (4.11) the deflection range can be increased by increasing either $L$ or $R / t$, both of which will reduce the system stiffness. A certain stiffness is important for the dynamic response. Furthermore, the leaf springs store the elastic deflection energy in whole spring length so that the components are less strained. The notch hinges hold the energy in very small local area, resulting in 
a high strain in the components. Therefore, from the points of view of deflection range and strain state, the leaf spring mechanism is superior to notch hinge mechanism.

\subsubsection{Parasitic deflection errors}

One of the advantages of using parallel spring mechanisms is that it produces a parallel or rectilinear displacement of a platform. If the specified performance is to be attained, attention has to be paid to some factors which may affect the accuracy of the performance of the mechanism.

\subsubsection{Effect of driving force}

The simple rectilinear mechanisms in Fig. 4.1 are assumed to be perfectly built, i.e. ignoring the manufacturing errors. When a horizontal driving force acts on a platform, the legs constrain each other to prevent end rotations: a tensile force built up in one, and a compressive force in the other to provide a moment that keeps the platform parallel. The forces in the legs will produce elastic distortions under the axial forces: the tensile force stretches the leg and the compressive force shortens the leg.

For the simple leaf spring rectilinear mechanism, possible motions of the platform are shown in Fig. 4.4. For a force $F$ applied as shown, the position of the moving platform can be described by the superposition of two deflexions, the intended parallelogram type of motion (Fig. 4.4(b)) and an undesired tilt due to cantilever type bending (Fig. 4.4(c)). The later produces a deviation from purely parallel motion. For small displacements, the pitching angle $\alpha_{\text {leaf }}$ of the platform is approximately given by [9]

$$
\alpha_{l e a f}=\left[\frac{2\left(L-2 L_{a}\right) d^{2}}{L_{b}^{2} L^{2}}\right] q
$$

where $L$ is the free length of leaf springs, $L_{a}$ is the height of acting line of force $F$, 
$L_{b}$ is the distance between two parallel leaf springs, $d$ is the thickness of springs and $q$ is the displacement of platform.

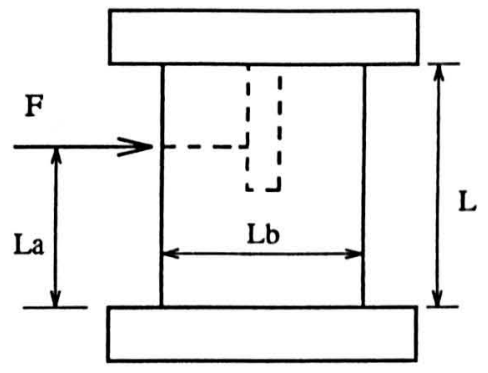

(a)

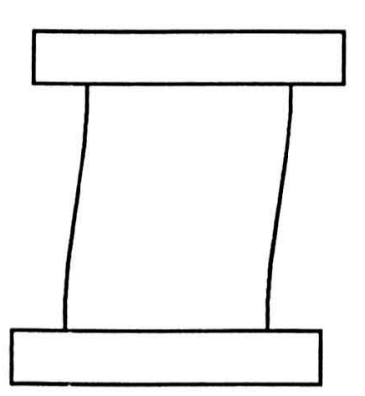

(b)

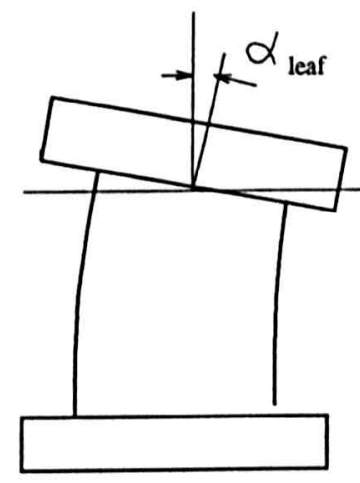

(c)

Figure 4.4: Simple parallel movement of leaf spring system: (a) undeflected, (b) intended parallel deflexion, (c) undesired deflexion

For simple notch hinge rectilinear mechanism, the pitching error caused by driving force can be considered in a similar way. The undesired distortions happen at four hinges resulting in a pitching angle $\alpha_{\text {hinge }}$ of the platform. The magnitude of the tensile or compressive force $R_{x}$ acted on each hinge can be worked out by (Appendix B)

$$
R_{x}=\frac{2 k_{z}\left(2 L_{a}-L\right)}{L^{2} L_{b}} q
$$

Then the pitching angle $\alpha_{\text {hinge }}$ of the platform can be written as

$$
\alpha_{\text {hinge }}=\frac{4 \Delta_{x}}{L_{b}}=\frac{4 R_{x}}{L_{b} k_{z}}
$$




$$
\begin{aligned}
& =\left[\frac{8\left(2 L_{a}-L\right) k_{z}}{L^{2} L_{b}^{2} k_{x}}\right] q \\
& =\left[\frac{16\left(2 L_{a}-L\right) t^{2}}{9 \pi L^{2} L_{b}^{2}}\left[\pi-2.57(t / R)^{1 / 2}\right]\right] q
\end{aligned}
$$

As an example the Table 4.1 gives a solution for the pitching angle models of equations (4.12) and (4.14), in which the two mechanisms have the same stiffness $k_{\text {s }}$ in motion translating direction. From the resultant pitchings it can be

\begin{tabular}{|c|c|c|c|c|c|c|}
\hline $\begin{array}{c}\text { Flexure } \\
\text { mechanisms }\end{array}$ & $R / t$ & $\begin{array}{c}d \\
(\mathrm{~mm})\end{array}$ & $\begin{array}{c}b \\
(\mathrm{~mm})\end{array}$ & $\begin{array}{c}q \\
(\mu \mathrm{m})\end{array}$ & $\begin{array}{c}k_{s} \\
(\mathrm{~N} / \mathrm{m})\end{array}$ & $\begin{array}{c}\alpha \\
(\mu \mathrm{rd})\end{array}$ \\
\hline Leaf spring & & 0.294 & 15 & 58 & 53.3 & 18.0 \\
\hline Hinge spring & $1 / 0.2$ & & 15 & 58 & 53.3 & 4.1 \\
\hline$L_{a}=14(\mathrm{~mm}), \quad L_{b}=10(\mathrm{~mm})$, & $L=10(\mathrm{~mm})$ \\
\hline
\end{tabular}

Table 4.1: Parasitic pitching errors caused by driving forces (from mathematic models)

\begin{tabular}{|c|c|c|c|c|c|c|c|}
\hline $\begin{array}{c}\text { Flexure } \\
\text { mechanisms }\end{array}$ & $R / t$ & $\begin{array}{c}d \\
(m m)\end{array}$ & $\begin{array}{c}b \\
(m m)\end{array}$ & $\begin{array}{c}q \\
(\mu m)\end{array}$ & $\begin{array}{c}k_{a} \\
(N / m)\end{array}$ & $\begin{array}{c}\alpha \\
(\mu r d)\end{array}$ & $\begin{array}{c}\sigma_{\max } \\
\left(N / m^{2}\right)\end{array}$ \\
\hline Leaf spring & & 0.3 & 15 & 57 & 52.9 & 18.9 & 38.9 \\
\hline Hinge spring & $1 / 0.2$ & & 15 & 58.1 & 51.7 & 10.0 & 72.6 \\
\hline$L_{a}=14(m m), \quad L_{b}=10(m m)$, & $L=10(m m)$ \\
\hline
\end{tabular}

Table 4.2: Parasitic pitching errors caused by driving forces (from FEA models)

seen that the performance accuracy of simple leaf spring rectilinear mechanism is poorer than an equivalent simple notch hinge rectilinear mechanism. From equation (4.12) and (4.14), the performance accuracy of both mechanisms can be improved by reducing the stiffnesses of the flexure elements and move down the acting lines of driving forces $F$. When the forces act midway along the springs, the pitching error is reduced to zero. These two pitching models were verified by Finite Element. Analysis. Fig. 4.5 and 4.6 give the FEA models and solutions with the numerical results listed in Table 4.2. 


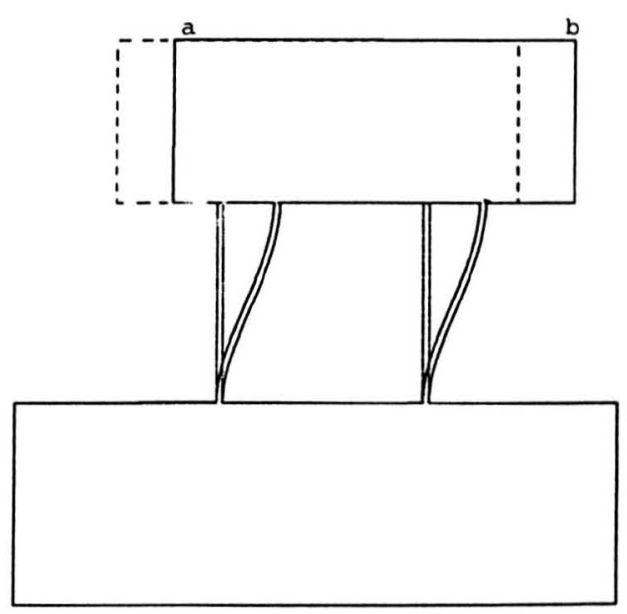

(a)

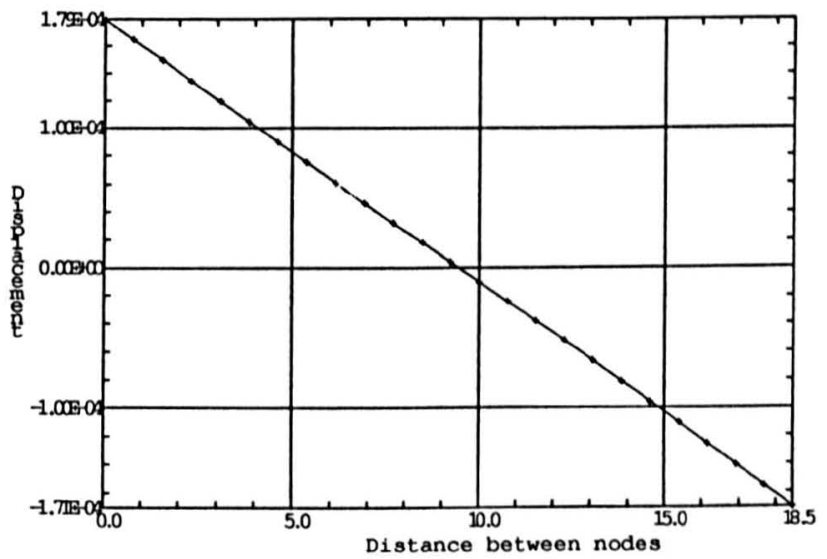

(b)

Figure 4.5: FEA model of leaf spring and its solution. (a) FEA model - deformed and undeformed, (b) Pitching curve of moving platform a-b. 


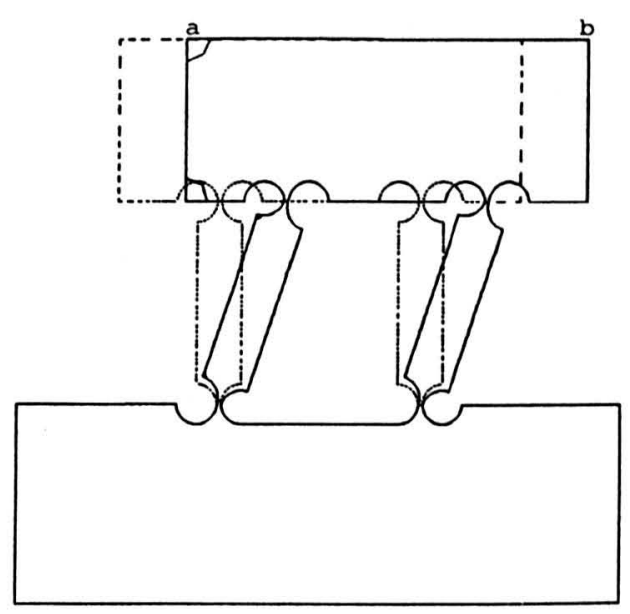

(a)

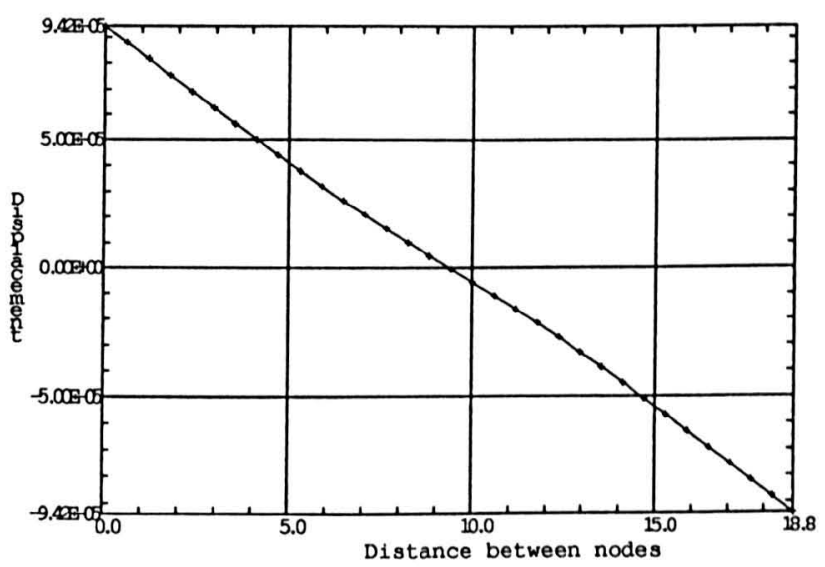

(b)

Figure 4.6: FEA model of hinge spring and its solution. (a) FEA model deformed and undeformed, (b) Pitching curve of moving platform a-b. 
Comparing the results in Table 4.1 and 4.2 , it can be seen that for leaf spring mechanism the result of mathematic model has a very good agreement with FEA. For hinge spring mechanism, the situation is more complicated because of the effects of stress concentration which makes the pitching error from FEA much bigger than that from mathematical model. By considering the effect of stress concentration on hinges the pitching error calculated with equation (4.14) becomes $6.9 \mu r d$. More importantly, misalignment of the driving force will cause rather large unexpected parasitic twisting. According to equation (4.6), the twisting error $\varphi$ of a single leaf spring can be estimated by

$$
\varphi=\frac{F z}{k_{t}}=\frac{12 L F z}{G d b^{3}}
$$

where $F$ is driving force, $z$ is the force misalignment distance from symmetric point. From this, it can be seen that the most sensitive parameter to the error is the width of spring. An error estimation done by Queensgate instruments Ltd. using computer modelling shows that a force acted at $7.5 \mathrm{~mm}$ away from the center of platform will produce a twisting error $\varphi=12 \mu \mathrm{rd}$ for a $50 \mu \mathrm{m}$ motion of simple notch hinge type rectilinear mechanism, parameters of which are given in Table 4.3 later in this section [10].

\subsubsection{Effect of manufacture}

Most of the simple parallel rectilinear spring systems used in earlier times were fabricated from many individual components. To achieve optimum performance requires a careful and systematic assembly. Fixing the component parts without introducing undesirable internal stresses and distortions is still a problem at assembly. It has been found with some flexures that there can now be a discrepancy of $30 \%$ or more between the theoretical and actual stiffness mainly due to the compliance of the screws and fixing plates [4]. Because of rapid advances in $\mathrm{CNC}$ machining techniques, monolithic mechanisms can be readily produced. Manufacture of notch hinge mechanisms is ideally suited to CNC milling and 
boring machines. Two holes are drilled close together in a solid blank and then excess material removed to reveal the desired shape. The accuracy of the hinge flexure is primarily dependent upon the accuracy of the centre of the holes with the material removed from the rest of the original blank being of little influence, so manufacture costs can be quite low. Manufacturing tolerances of these hinges always degrades performance of the platform from the geometric ideal. There are three sources of potentially significant errors, Fig. 4.7. The first two are the errors in the $x-y$ plane, which are similar to the cases analyzed by Jones [9] on leaf spring mechanism. When hinge separations on two legs differ by $\epsilon_{a}$, i.e. one leg length is $L$ and the other $L+\epsilon_{a}$, as shown in Fig. 4.7 (a), the parasitic pitching error about the $z$ axis can be approximately given by

$$
\theta_{a} \approx \frac{\epsilon_{a} x^{2}}{2 L^{2} L_{b}}
$$

where $x$ is platform motion along the $x$ axis, and $L_{b}$ is the nominal separation between two legs. In the other case Fig. 4.7(b), where two legs are not parallel due to error $\epsilon_{b}$ between the separations of the two legs $L_{b}$ on the platform end and base end, the parasitic pitching error is then

$$
\theta_{b} \approx \frac{\epsilon_{b} x}{L L_{b}}
$$

For general precision devices, displacement $x$ is always very small compared with the geometric parameters of rectilinear mechanism. Then among the two equations above, the later will predominate. The third case is that the hinges are not machined vertical to the moving plane with a deviation angle $\epsilon_{c}$ from the $x$ axis, i.e. they are not parallel in the $x-z$ plane. This will cause an unexpected platform rotation about the $y$ axis, as shown in Fig. 4.7(c). The parasitic yawing $\theta_{c}^{*}$ can be geometrically worked out as

$$
\theta_{c}^{*} \approx \frac{x \epsilon_{c}}{L_{b}}
$$




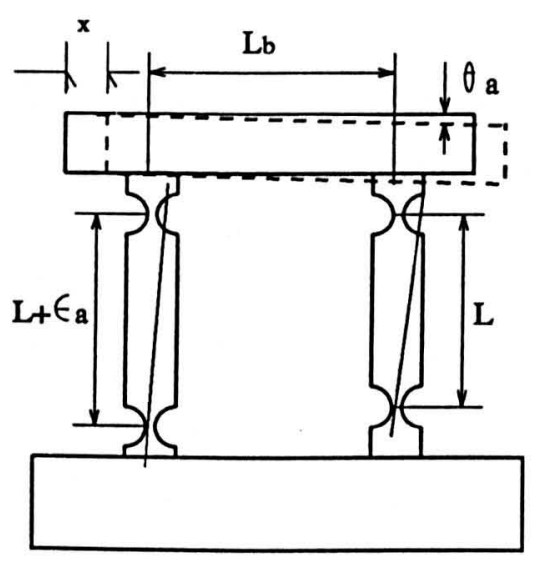

(a)

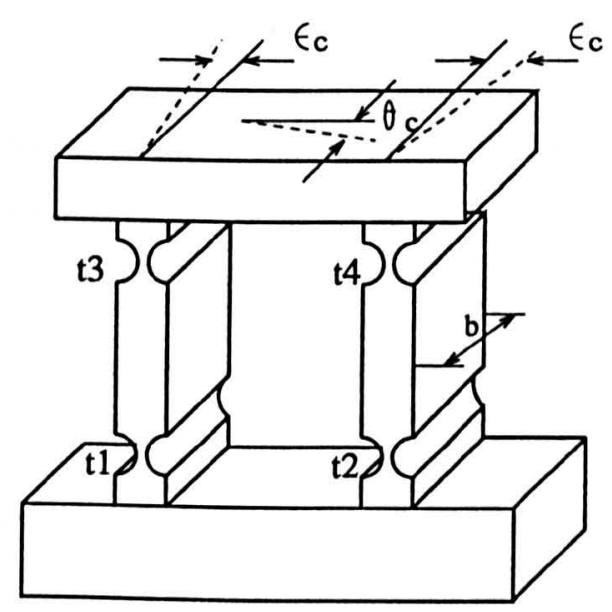

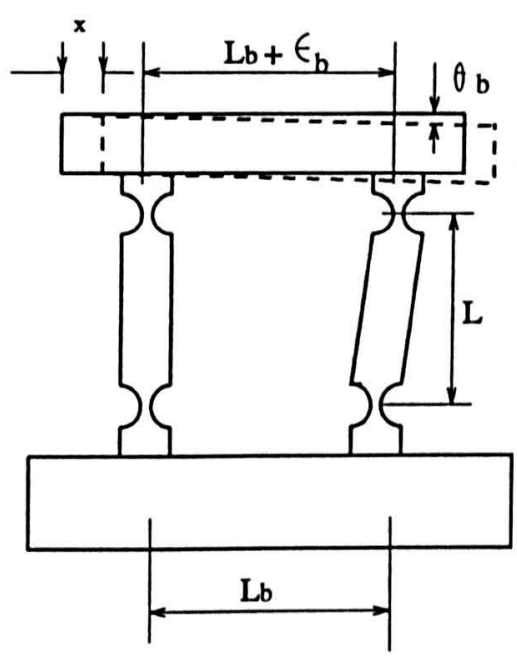

(b)

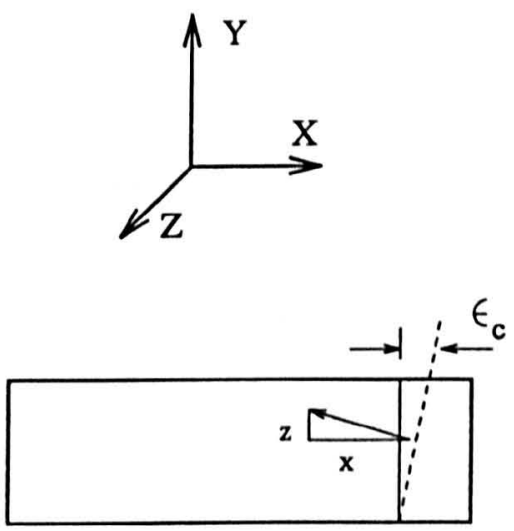

(c)

Figure 4.7: Deflexion from parallelism of motion caused by manufacturing errors 
However, the two legs connected to the platform will restrain each other rotating about the $y$ axis. Because they have same stiffness, the actual parasitic yawing $\theta_{c}$ will be reduced by half

$$
\theta_{c} \approx \frac{x \epsilon_{c}}{2 L_{b}}
$$

The investigation to this case has recently been done by Queensgate Instruments Ltd. using computer modelling with finite element analysis [10], Table 4.3. If

\begin{tabular}{|c|c|c|c|c|c|}
\hline Case & \multicolumn{4}{|c|}{$\epsilon_{c}(m r d)$} & Error $\theta_{c}(\mu r d)$ \\
\hline & $\epsilon_{c} 1(m r d)$ & $\overline{\epsilon_{c} 2(m r d)}$ & $\epsilon_{c} 3(m r d)$ & $\epsilon_{c} 4(m r d)$ & \\
\hline $\mathrm{cl}$ & 17 & 0 & 17 & 0 & +9.6 \\
\hline $\mathrm{c} 2$ & 17 & -17 & 17 & .17 & +20 \\
\hline $\begin{array}{l}t=0 \\
L= \\
x= \\
\epsilon_{c} 1, \epsilon\end{array}$ & $\begin{array}{l}2 m, K \\
2 m, L_{b} \\
m, \text { pla } \\
\varepsilon_{c} 3, \epsilon_{c}^{4}\end{array}$ & $\begin{array}{l}m, \text { hin } \\
m m, b \\
\text { the erro } \\
\text { the }\end{array}$ & $\begin{array}{l}\text { s thickn } \\
5 \mathrm{~mm} \\
\text { n the } x \\
\text { on hing }\end{array}$ & nd radius & \\
\hline
\end{tabular}

Table 4.3: Rotation error of a simple flexure caused by non-parallel arrangement of parallel hinges

the same pararneters in Table 4.3 (case $c 1$ ) are brought into equation (4.19), one can obtain $\theta_{c}=10.6 \mu r d$ which is very close to the result of computer modelling. Bringing the parameters in Table 4.3 into equation (4.16)and (4.17) with $\epsilon_{a}=$ $\epsilon_{b}=1 \mathrm{~mm}$, the parasitic pitching errors are calculated to be $\theta_{a}=0.046 \mu r d$ and $\theta_{b}=48.1 \mu r d$, respectively. $\epsilon_{a}=\epsilon_{b}=1 \mathrm{~mm}$ and $\epsilon_{c}=17 \mathrm{mrd}$ are huge errors for CNC machining, therefore, from the results it can be seen that the effects of manufacture errors on the performances of platform are very small, pitching and yawing errors being less than $10^{-3}$ of manufacturing errors.

\subsubsection{Compound rectilinear mechanisms}

The simple rectilinear mechanism discussed above has the disadvantage of intrinsic parasitic motion perpendicular to the traverse direction. In some applications this motion, although very small, is not acceptable. Using two such motions in 
a compound design, Fig. 4.8, eliminates the error. The double compound rectilinear mechanism is simply two simple compound rectilinear mechanism joined together about the $x$ axis, Fig. 4.9. The symmetrical structure gives a symmetrical stress distribution on both platform and base frame. The platform is restrained by flexure legs on both sides, an extremely precise rectilinear motion along $x$ axis can be achieved. The distortions of both platform and base frame will be much smaller than other rectilinear mechanisms due to the symmetrical stress distribution by which the bending moments on the platform or base frame cancel each other. The other advantage of using this type of mechanism is that there will not be any moment force transferred from the system to the external mechanisms on which it is mounted. This characteristic is particularly important for application in two dimensional precision rectilinear translation mechanism, in which the moment force from the stage moving in the $x$ axis will affect the performance the other stage moving in the $y$ axis. Further more, the symmetrical design is obviously less susceptible to the influence of thermal effects. Therefore, the symmetrical structure has been commonly used in precision $x-y$ positioning and scanning stages. For the scanning stage used in modern microscope, a compact structure is essential for high dynamic response and vacuum availability. In practice, several variations of double compound spring systems have been developed. The para-flex stage deviced by Teague and his colleagues, Fig. 4.10, uses three-hole/two-web flexure elements to form a symmetric restrain to platform, so that the forces transferred to its frame are symmetric and moment forces are cancelled by themselves [11]. Another example is double leaf spring hinge used recently in some commercial $x-y$ scanning stages, Fig. 4.11.

\subsubsection{Two dimensional rectilinear mechanisms}

Two-axis linear systems can be generally considered as combinations of two oneaxis linear systems discussed above. There are two basic configurations for two dimensional rectilinear mechanisms, symmetric structure and asymmetric struc- 


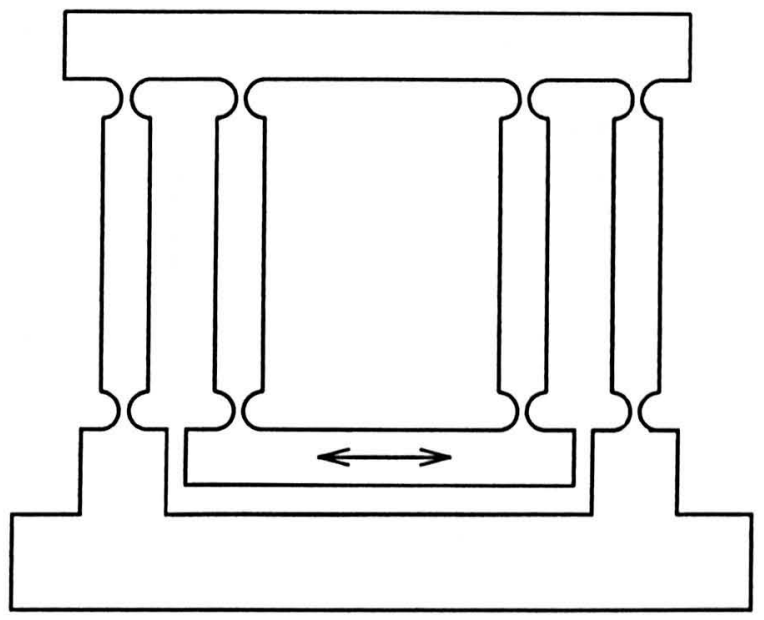

Figure 4.8: Schematic diagram of compound rectilinear mechanism

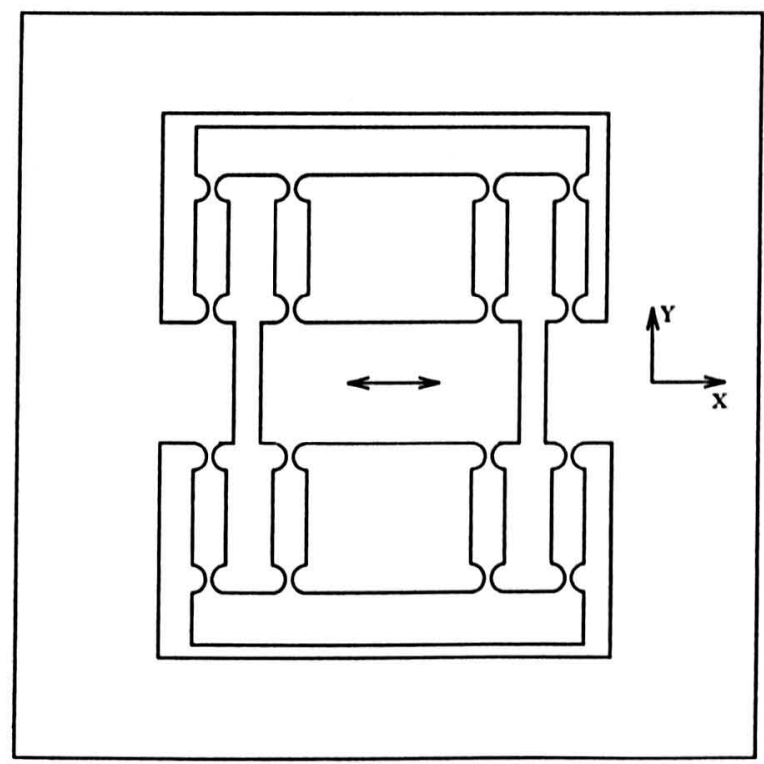

Figure 4.9: Schematic diagram of double compound rectilinear mechanism 


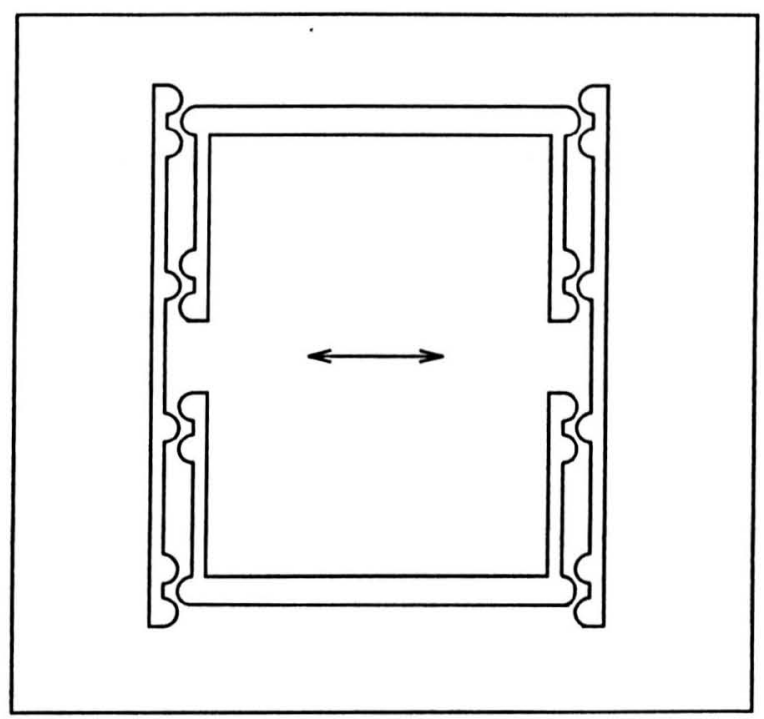

Figure 4.10: Schematic diagram of a symmetric rectilinear system of three-hole/two-web flexure elements

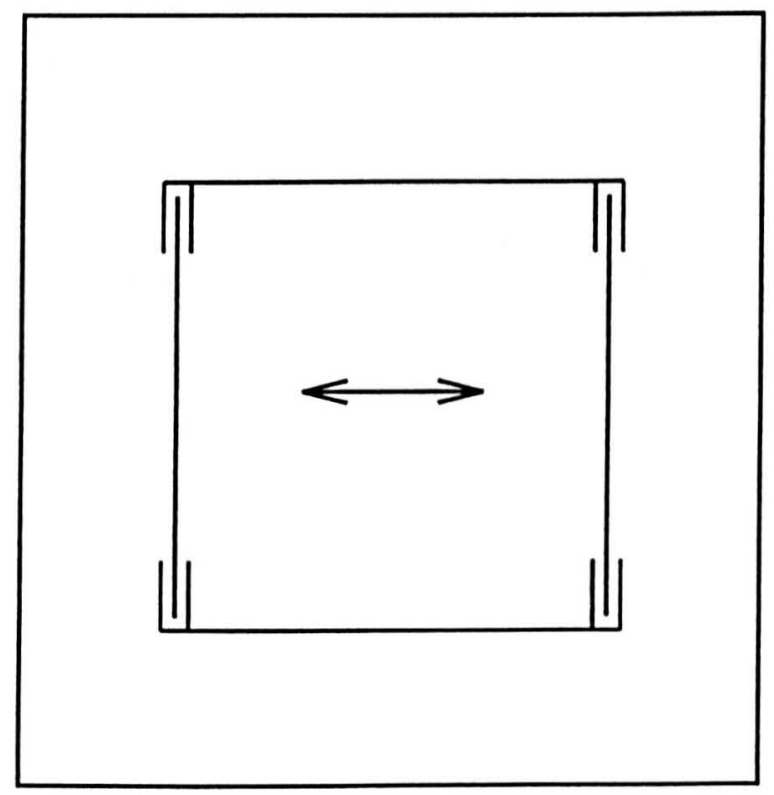

Figure 4.11: Schematic diagram of a symmetric rectilinear system double leaf spring flexures 
ture as shown in Fig. 4.12. The merits of the symmetrical mechanism can be summarized as

(1) It has higher accuracy in its motion. Because double compound spring system has to be used, theoretically, there is no intrinsic error.

(2) The system has a symmetrical stress distribution. The forces transferred from platform to frames are symmetrically balanced and the moment forces are balanced locally, so that the frames are distorted symmetrically, which will have less effects on the motion of platform.

(3) The symmetrical structure is less susceptible to thermal effects.

(4) It is capable of supporting heavy specimens because the platform is closely connected to the base on its four corners.

The main advantage of asymmetrical mechanism is that it is easy to build the system very compact in structure, especially for the piezo actuator driven monolithic translation mechanism. The piezo actuators for driving in both the $x$ and $y$ axes can be seated outside of the translation system in the solid block, keeping far away from the platform. In this case, the driving forces can be directly transferred to the solid frame, producing very small influence on the translation system. Besides, its compactness in structure makes it superior in dynamic characteristics which is also very important for the precision mechanism with these concerns. The $x-y$ scanning stage which will be introduced later is an example of the application of this configuration. 


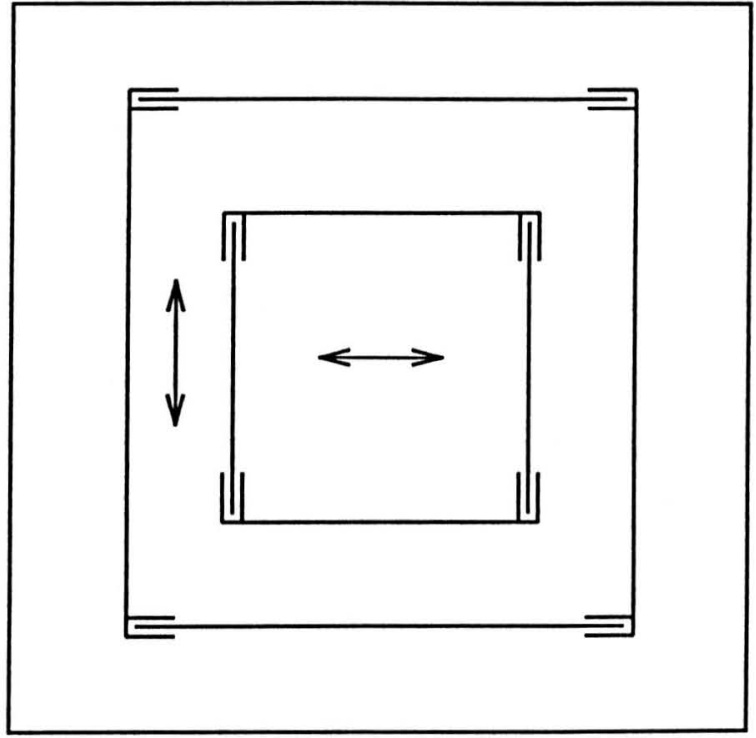

(a)

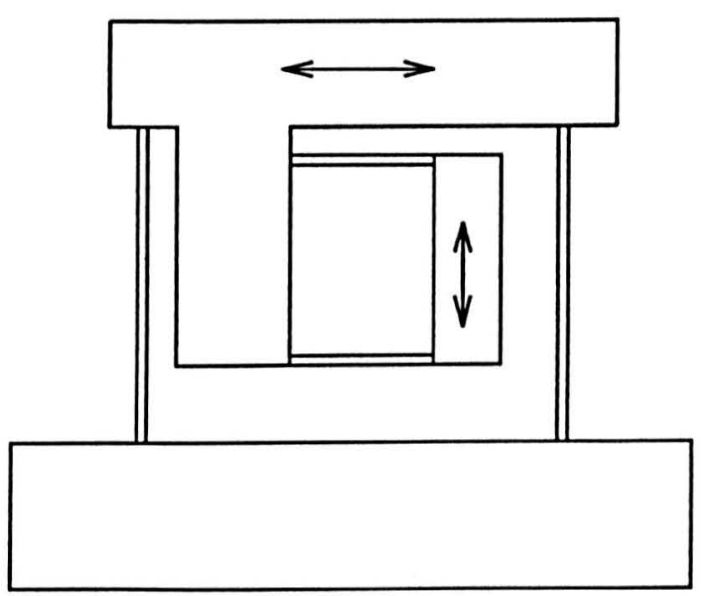

(b)

Figure 4.12: Schematic diagram of two dimension rectilinear mechanism 


\subsection{Design of precision $X-Y$ scanning stage for SPM}

\subsubsection{Introduction}

To meet the special requirements of the new generation of scanning probe microscopes, Queensgate Instruments Ltd. in U.K. is, in recent years, developing a serious of stages for precision positioning and scanning. The author joined the project as one of the designers. As an important part of our metrological scanning force microscope, the $S 220 x-y$ scanning stage has been built jointly by Queensgate Instruments Ltd. and the Centre for Nanotechnology and Microengineering at the University of Warwick. The rest of this chapter gives the details of designing, building and performance assessment of the stage, and concludes with some discussions.

The main objectives to be achieved for the design of the stage are:

(1) Positioning and scanning range $50 \mu m$ in both the $x$ and $y$ axes with potential resolution at nanometer or subnanometer levels.

(2) Resonant frequency of the system $>500 \mathrm{H}_{x}$.

(3) Closed loop control with high linearity, insignificant hysteresis and low drift.

(4) Low crosstalk for open loop application.

(5)Compact.

\subsubsection{Description of the X-Y stage}

Fig. 4.13 shows a diagram of the $S 220 x-y$ stage, which consists mainly of stage body, actuators and position sensors. A group of flexure pivoted levers amplify the translation of the PZT actuators. There are two piezoelectric actuators embedded in the stage to provide the driving force for each axis. The expansion 
range of the PZT stacks is about $15 \mu \mathrm{m}$ for a drive voltage of $100 \mathrm{~V}$. To move the platform in the $x$ axis, the corresponding piezo actuator pushs lever 1 to rotate about hinge $a$ (see Fig. 4.14). The lever 1, in turn, pulls the platform 3 to move along the $x$ axis through a flexure arm 2. The expansion of the actuator is amplified by lever 1 by a ratio of 1:4. In the same way, the expansion of the actuator for the $y$ axis is transmitted through lever 6 to the platform support 7. Two parallel notch hinge linked levers $4 \& 5$ in each side of the platform constrain it moving rectilinearly along orthogonal $x$ and $y$ axes. All the levers and notch hinges are monolithically machined in an aluminium alloy block using wire electro-discharge machining.

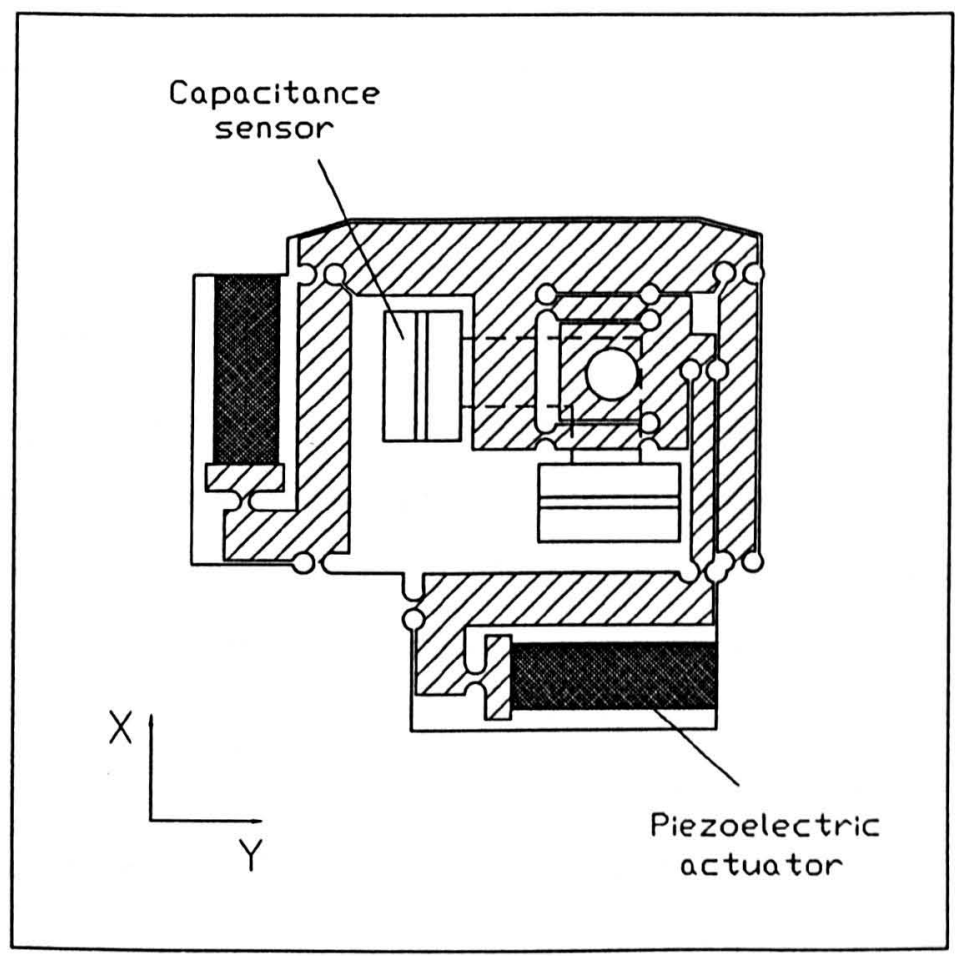

Figure 4.13: Schematic diagram of configuration of $S 220 x-y$ scanning stage

Two pairs of capacitance sensors are mounted in the stage for monitoring the 


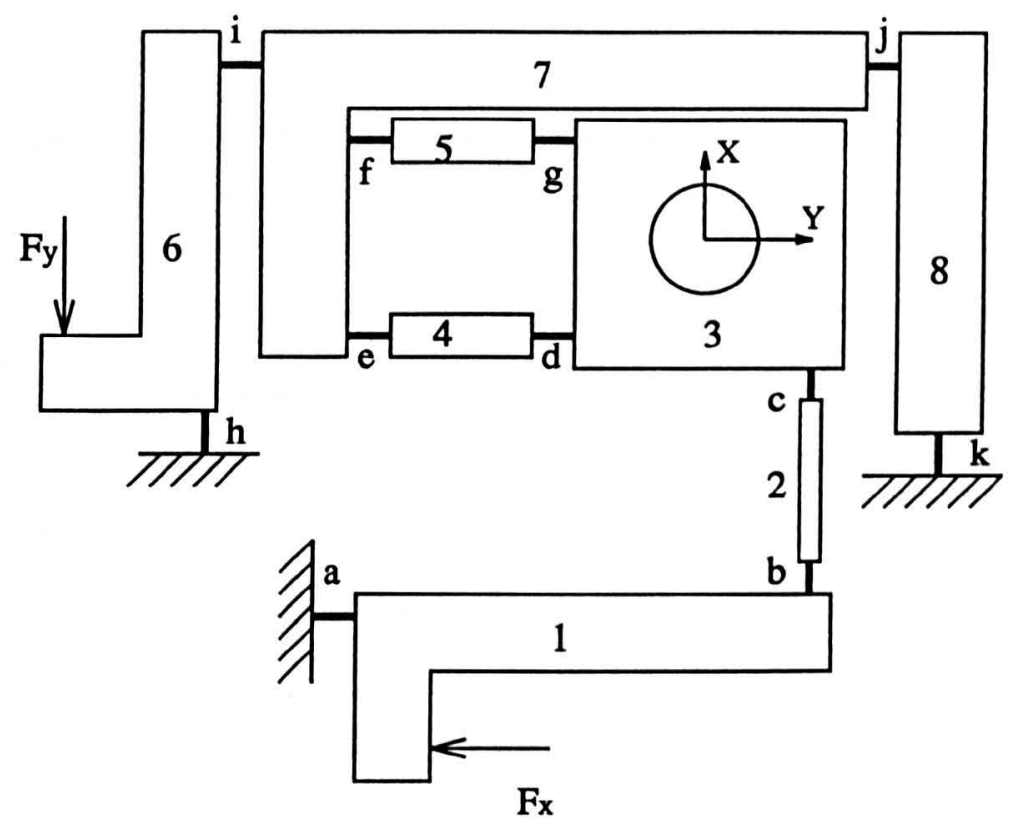

Figure 4.14: Schematic diagram of translation configuration of the $x-y$ scanning stage

actual position of the platform in the two axes. The capacitance gauge, Nanosensor (NS2000), used here is produced by Queensgate Instruments Ltd. This kind of gauge can resolve to $<1$ nanometer over 500 microns of displacement, with a bandwidth of $5 \mathrm{kHz}$. It comprises of two main parts: two sensor electrodes which form a parallel plate capacitor when attached to the pieces to be measured and an electronics module which measures the change in the capacitor's impedance as a function of the distance between the electrodes. One of the electrode plates is mounted on and moves together with the platform, and the other one is fixed at the base block on which the stage is held. The rectilinear moving of the platform will lead to relative change in distance between two electrodes.

The size of the stage body is $60 \times 56 \times 15 \mathrm{~mm}$, and it is kinematically mounted on an aluminium base. The surface of the base is used as datum on which the target electrodes of the capacitance sensors are fixed. The specimen is firstly fixed on a sample holder using double glue tape. Then the holder is positioned on the 
platform through a short taper bar on the holder and a hole in the platform.

\subsubsection{Some problems in the design}

Most of today's precision scanning stages use piezoelectric or electrostrictive components as actuators. It is well known that piezoelectric transducers (PZT) are easy to control with nanometric resolution. They have good dynamic properties and are capable of driving a high load. The main disadvantage of using it is that the amount of expansion is very small, only a $\mu \mathrm{m}$ per $\mathrm{mm}$ of the stack in most practical applications. To achieve long range displacement, two options of design strategy are generally considered, driving the stage with a multi-stack PZT which can produce large expansion, or using a large lever ratio to amplify the expansion of the actuators. The former is simple in structure, but much more expensive and not compact needing a large space to fit in the multi-stack actuators. The later is adopted in our design, so that the structure of the stage can be made very compact and the stiffness loss of multi-stack systems is avoided.

For scanning stages used for scanning probe microscopes, much attention should be paid to its dynamic properties. A high resonant frequency will not only provide a wide working bandwidth, but also render the stage less susceptible to the disturbance of environment. All the scanning probes are extremely sensitive sensors, which are correspondingly sensitive to environmental influences. The shorter the measuring time, the smaller the environment influence will be. Therefore, a certain scanning speed is essential for a good measurement. The stability of mechanical systems is crucial for a scanning stage to work reliably at nanometer levels. Some researchers met the problem that it was very hard to use the flexure stage of low resonant frequency in their SPM to achieve expected results due to the vibration problem [12]. The natural frequency of a mechanical system can be simply calculated by

$$
\omega_{n}=\sqrt{\frac{k_{e}}{m_{e}}}
$$


where $k_{e}$ is effective stiffness and $m_{e}$ is effective mass. Natural frequencies can be increased by either reducing the mass or increasing the stiffness. By making the structure very compact, the mass can be reduced. However, considering the fixed mass of sample holder and specimen, the improvement is quite limited. Alternatively, the system stiffness has to be increased. The system stiffness is determined by the individual stiffnesses of each spring flexure element and their distribution in the translation mechanism. High stiffness introduces high driving force, which is always a problem for precision mechanisms. The stage may be distorted and its performance deteriorated easily by these driving forces, especially for the lever mechanisms of motion amplifying mode in which the driving forces will be amplified in proportion to magnification of the motion. Another effect of the system stiffness which has to be considered in the design is the loss of motion if the stiffness difference between the spring system and piezo actuator is not big enough. Taking piezo actuator and translation mechanism as a serial spring system, the relative loss of the actuator's expansion will be

$$
\frac{\Delta_{p}}{\Delta}=\frac{k_{1}}{k_{1}+k_{2}}
$$

where $\Delta_{p}$ is the expansion loss of actuator, $\Delta$ is the expected expansion, $k_{1}$ and $k_{2}$ are the stiffness of the translation mechanism and the actuator, respectively. For stiffness values of $k_{1}=2 \mathrm{~N} / \mu \mathrm{m}, k_{2}=8 \mathrm{~N} / \mu \mathrm{m}$ (a common stiffness for commercial piezoelectric stacks), a $20 \%$ loss of motion is predicted. Usually, the stiffness of piezo stacks has a dominating influence on the dynamic properties of the stage because its stiffness is much higher than the stiffness of the flexures in deflection directions. For the stage of translation mechanism of motion amplification mode, the effective stiffness of the piezo stack to the scanning platform will be reduced significantly. According to the simple model in Fig. 4.15, the effective stiffness 
can be calculated by

$$
\begin{aligned}
& \Delta=\Delta_{\text {piezo }} Q=\left(\frac{Q F}{k_{\text {piezo }}}\right) Q \\
& k_{e}=\frac{F}{\Delta}=\frac{k_{\text {piezo }}}{Q^{2}}
\end{aligned}
$$

where $Q$ is the magnification of motion. From the discussion above it is obvious

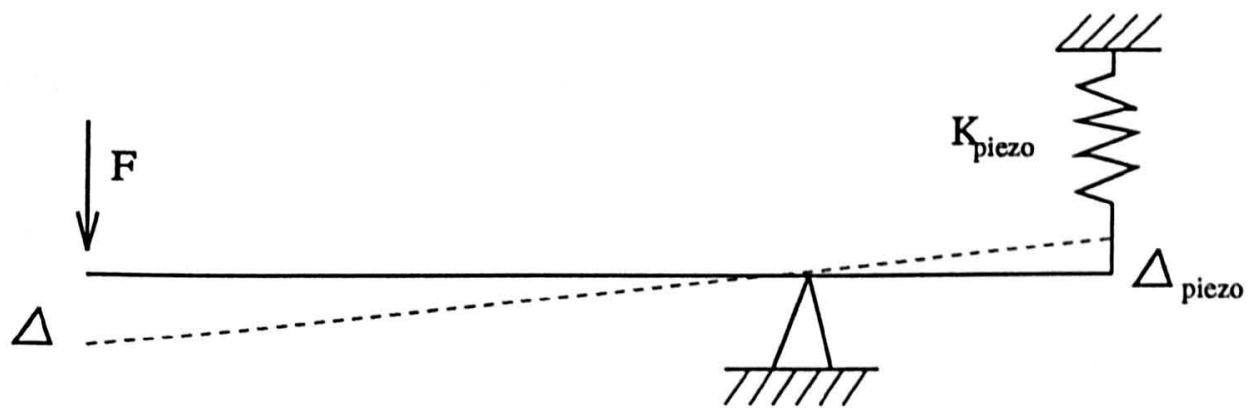

Figure 4.15: Schematic diagram of a simple model of motion amplification mechanism for effective stiffness analysis

that for the design of a motion amplifying translation mechanism, the magnification is mainly limited by the stiffness of piezo actuators in both static and dynamic aspects.

It is well known that the piezoelectric actuator has inherent non-linearities such as hysteresis and creep in voltage-displacement characteristics. Although, in principle, it is possible to reduce these effects by applying a compensation voltage, in practice these techniques have shown to be impracticable thus far. The alternative strategy we employed is to monitor the actual position of the stage in situ with a precision capacitance gauge. This enables the implementation of either open loop monitoring or closed loop controlling of sample motion. In the former case, it is not necessary for actuator controllers to keep scanning in raster fashion very precisely. So high speed scanning becomes possible and undistorted profiles and images can be obtained by sampling the probe and the position gauges simultaneously. In this case, the data have to be gridded with 
interpolation before analyzing and graphing. The latter mode is more usually used, with which the output of position gauges is used as feedback signal for servo control to position the stage accurately and to scan in a precise raster pattern. In this way, the process of image producing is much simplified and the fidelity of profile will be increased when used in scanning microscopes.

\subsubsection{Dynamic modelling}

To simplify the the model for analysis, the compound spring system of the stage can be divided into two separate parts in accordance with the scanning axes, $x$ and $y$ axes. Fig 4.16 is a schematic diagram of these two subsystems, both of

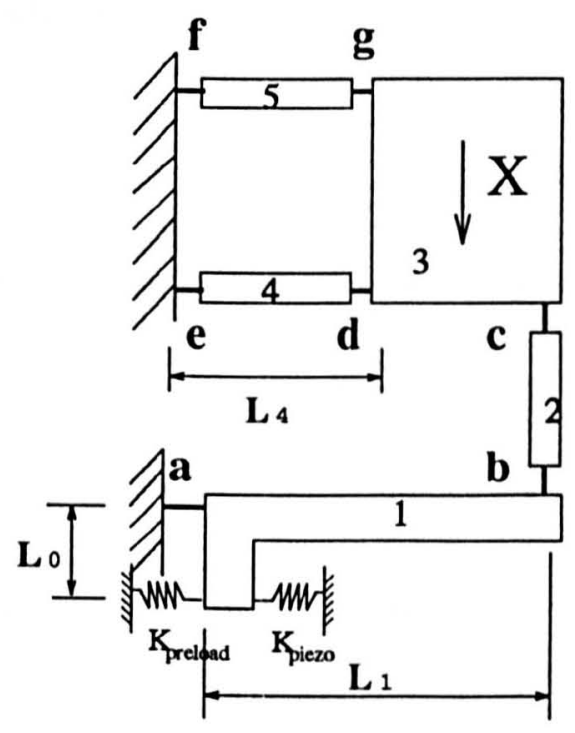

(a)

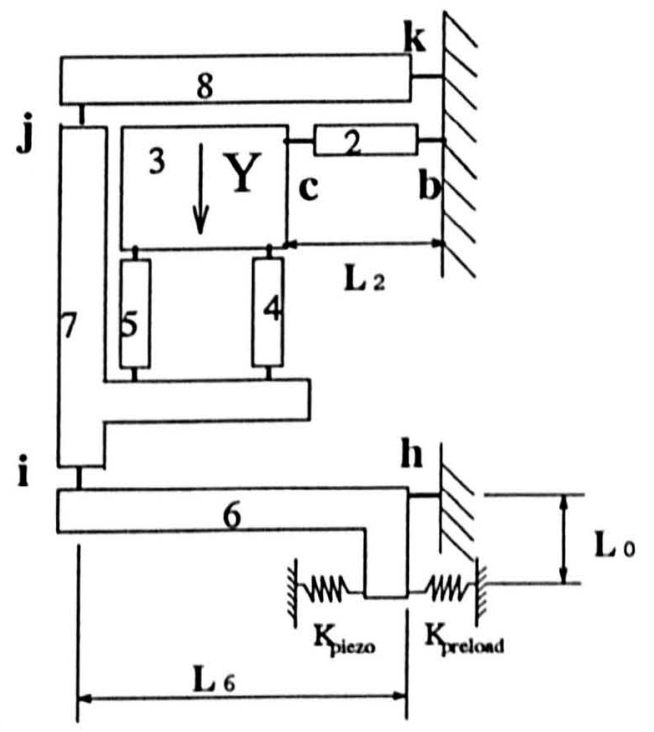

( b )

Figure 4.16: The schematic diagram of spring systems on the $x$ and $y$ axes. (a) Model for the $x$ axis, (b) Model for the $y$ axis

which are of single mobility. Here we assume that all levers are rigid components and all flexure pivots do not stretch and compress and the stiffness of each hinge along the axis of the lever to which it is connected is extremely high compared with that in its deflection direction. Fig. 4.16 (a) shows spring system of $x$ axis. 
For a small displacement, it can be considered as a linear spring system. Total mechanical energy of the system is the sum of kinematic energy $T$ of lumped masses, and potential energy $V$ stored in hinge springs, given by

$$
\begin{aligned}
& T=\frac{1}{2} I_{1} \dot{\theta}_{1}^{2}+\frac{1}{2}\left(I_{4}+I_{5}\right) \dot{\theta}_{4}^{2}+\frac{1}{2}\left(m_{2}+m_{3}\right) \dot{x}^{2} \\
& V=\frac{1}{2} k_{p} \theta_{1}^{2} l_{0}^{2}+\frac{1}{2} k_{a} \theta_{1}{ }^{2}+\frac{1}{2}\left(k_{d}+k_{e}+k_{f}+k_{g}\right) \theta_{4}^{2}
\end{aligned}
$$

where $\theta_{1}, \theta_{4}$ are the rotation angles of lever 1 and $4 . I_{1}, I_{4}$ and $I_{5}$ are the second moments of the masses of element 1,4 and $5, m_{2}, m_{3}$ are the mass of elements 2 and $3, k_{p}$ is the stiffness of piezo actuator, $k_{a}, k_{d}, k_{e}, k_{f}$ and $k_{g}$ are the rotation stiffness of hinge flexures $a, d, e, f$ and $g$ respectively, here $k_{d}=k_{e}=k_{f}=k_{g}$. Defining the Lagrangian $L=(T-V)$ and using Lagrange's equation of motion

$$
\sum_{i=1}^{n}\left\{\frac{d}{d t}\left(\frac{\partial L}{\partial \dot{q}_{i}}\right)-\frac{\partial L}{\partial q_{i}}=Q_{i}\right\}
$$

As the result, the natural angular frequency $\omega_{n x}$ of the stage is obtained

$$
\omega_{n x}=\left(\frac{k_{p} l_{0}^{2}+k_{a}+4 k_{d}\left(\frac{l_{1}}{l_{4}}\right)^{2}}{I_{1}+\left(m_{2}+m_{3}\right) l_{1}^{2}}\right)^{\frac{1}{2}}
$$

The natural periodical frequency is then given by

$$
f_{n x}=\frac{\omega_{n x}}{2 \pi}
$$

The natural frequency of the stage in the $y$ axis $f_{n y}$ can be derived in the same way

$$
\begin{aligned}
& T=\frac{1}{2} I_{6} \dot{\theta}_{6}^{2}+\frac{1}{2} I_{8} \dot{\theta}_{6}^{2}+\frac{1}{2} I_{2} \dot{\theta}_{2}^{2}+\frac{1}{2}\left(m_{3}+m_{4}+m_{5}+m_{7}\right) \dot{y}^{2} \\
& V=\frac{1}{2} k_{p} \theta_{6}^{2} l_{0}^{2}+\frac{1}{2}\left(k_{h}+k_{i}+k_{j}+k_{k}\right) \theta_{6}^{2}+\frac{1}{2}\left(k_{b}+k_{c}\right) \theta_{2}^{2}
\end{aligned}
$$


then

$$
\begin{gathered}
\omega_{n y}=\left(\frac{k_{p} l_{0}^{2}+k_{g}+k_{f}+k_{d}+k_{e}+2 k_{b} l_{6}^{2} / l_{2}^{2}}{I_{6}+I_{8}+I_{2} l_{6}^{2} / l_{2}^{2}+\left(m_{3}+m_{4}+m_{5}+m_{7}\right) l_{6}^{2}}\right)^{\frac{1}{2}} \\
f_{n y}=\frac{\omega_{n y}}{2 \pi}
\end{gathered}
$$

where $k_{b}, k_{c}, k_{h}, k_{i}, k_{j}$ and $k_{k}$ are the rotation stiffness of notch hinge $b, c, h, i, j$ and $k$, respectively, here $k_{b}=k_{c}, I_{2}, I_{6}$ and $I_{8}$ are the second moments of the masses of levers 2,6 and $8, m_{3}, m_{4}, m_{5}$ and $m_{7}$ are the masses of elements 2 and 3 , and $\omega_{n y}$ and $f_{n y}$ are the natural angular and periodical frequencies of the stage in $y$ axis.

Bringing the parameters of the stage $S 220$ into the equations above, the natural frequencies of the system are obtained, $f_{x}=1267.0 H_{z}, f y=818.1 H_{s}$. These are calculated in the condition that there is no additional mass loaded on the platform. If the masses of sample holder and specimen are considered, the natural frequencies of the system will decrease correspondingly. Fig. 4.17 shows the curves of natural frequencies vs mass added on the platform. The figure illustrates that the frequency in the $x$ axis drops much quicker than in the $y$ axis as the mass increases. The reason is that the mass of the translation system in the $x$ axis is smaller than that in the $y$ axis. The smaller the mass of translation mechanism, the larger the effect will be.

\subsubsection{Experimental assessment of motion errors}

From the kinematic point of view, the performance accuracy of the $x-y$ stage can be affected by errors coming from all components of movability, such as the errors of axial displacement, straightness and linearity in the $x$ and $y$ axes, and yaw, pitch and roll. For the stage (S220), the errors happen in the axial directions are controllable using closed loop control mode, so that the yaw, pitch and roll are considered to be the major kinematic error sources. A large Abbe error may be produced if the measurement axis is not coincident with the platform center. For example, if a measurement axis is $5 \mathrm{~mm}$ from the platform center, $1 \mu \mathrm{rd}$ rotation 


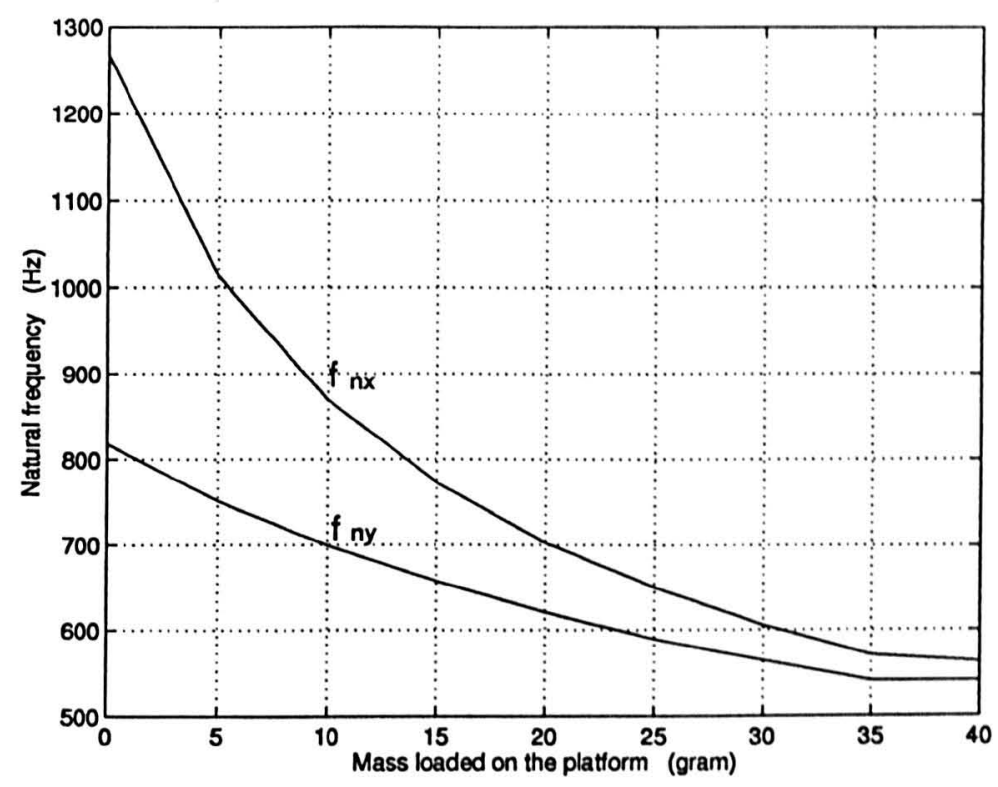

Figure 4.17: The relationship between natural frequency and mass added on the platform.

of the platform will give an error of $5 \mathrm{~nm}$ in tip position. Theoretically, the error can be easily compensated in data processing if the distance between measuring point and platform center is known. However it is not practical to measure this distance. So, the errors have to be reduced as much as possible in the design.

To determine the magnitudes of these errors, the yaw, pitch and roll were measured with optical lever and photodiode. The measurement setup for these experiments is shown in Fig. 4.18. A laser beam which passes through a beam splitter is reflected by a small mirror set on the centre of the platform. The reflected beam is partially reflected again by the beam splitter and acts on a photodiode. The photodiode is mounted on a stage which can be moved manually by micrometers in vertical and horizontal directions. Its position is monitored with an LVDT position gauge. Before driving the $x-y$ stage, the photodiode is nulled in both vertical and horizontal axes. By moving the platform in the $x$ axis, the yaw and pitch will change the beam's reflecting directions, resulting in a change in the output of the photodiode. Adjusting the diode position to null 


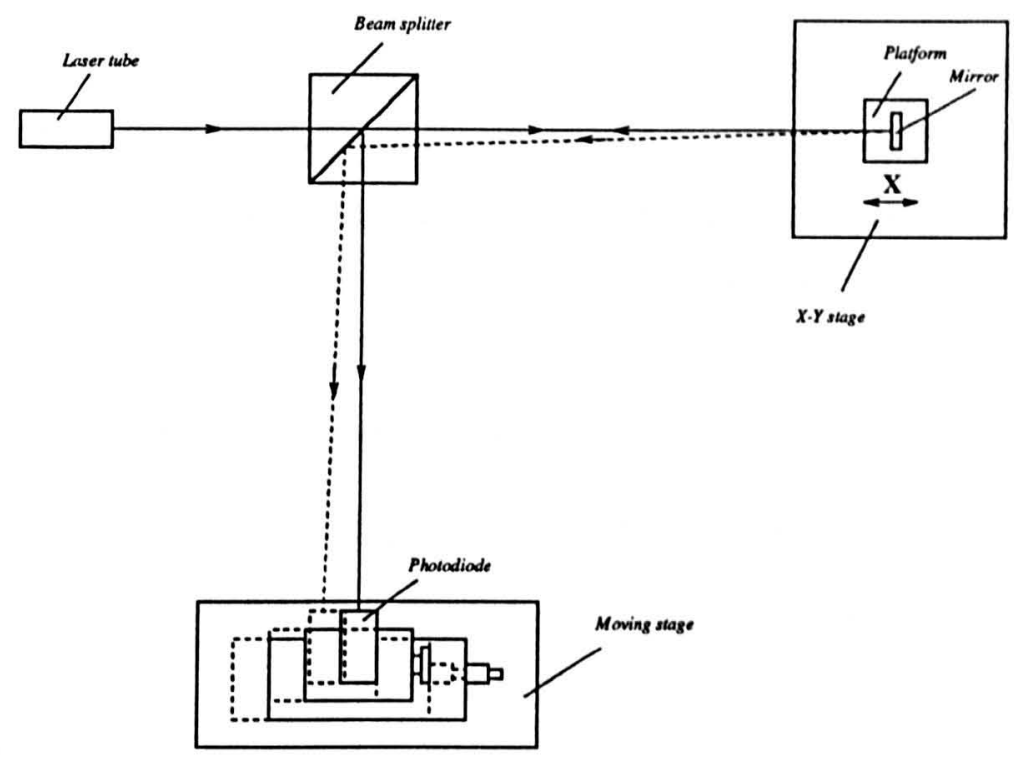

Figure 4.18: The schematic diagram of angular error measurement setup for measuring the yaw and pitch errors of the $x$ axis and the roll error of the $y$ axis

it again. The yaw and pitch errors in the $x$ axis can be calculated out according to the diode's displacement monitored by LVDTs in horizontal and vertical axes, respectively. When the $x-y$ stage is in the state as shown in Fig. 4.18, by driving the platform in the $x$ axis the yaw and pitch of the $x$ axis is measured and by driving the platform in the $y$ axis the roll and yaw in the $y$ axis are measured. Rotating the $x-y$ stage by $90^{\circ}$, the yaw and pitch errors in the $y$ axis and the roll error in the $x$ axis can be measured in the same way. The measurement results are shown in Table 4.4. The sensitivity of the photodiode to position change of beam

\begin{tabular}{|c|c|c|c|}
\hline Axis & Yaw $(\mu r d)$ & Pitch $(\mu r d)$ & Roll $(\mu r d)$ \\
\hline$X$ & 508 & 43 & 8 \\
\hline $\mathrm{Y}$ & 286 & 23 & 8 \\
\hline \multicolumn{4}{|l|}{ The measurements were taken over a scanning distance of $50 \mu \mathrm{m}$} \\
\hline
\end{tabular}

Table 4.4: Measured results for angular errors of the $x-y$ stage 
$m$ used in these experiments. The fluctuation of voltage output is within $2 \mu V$, which may be affected by the stability of laser beam, atmospheric variations and electronic circuit. The drift of the system is found to be reasonably constant, so its influence cau be eliminated by averaging the results of several measurements in which the platform is moved forwards and backwards alternately.

\subsubsection{Finite element analysis}

To optimize the design and investigate the possible error sources of performance, a finite element analysis (FEA) was carried out, both static and dynamic solutions being calculated. The FEA work was done using I-deas package on a SPARC Sun work station.

\subsubsection{Geometrical model building and mesh generation}

I-deas has very good graphic processing capability. The most outstanding characteristic of the package is its capability of automatic mesh generating. To build a finite element analysis model for such a complicated shape of the $x-y$ stage is very difficult and certainly is not feasible to do it manually. The geometrical characteristic of the stage, to which much attention has to be paid in mesh generating, is that the dimensions of components vary significantly in certain regions. At hinge areas, minimum thickness of web is as small as $0.1 \mathrm{~mm}$ and curvatures are fairly sharp, so very fine elements are needed. However, in other areas like thick frame of the stage being tens of millimetres in dimension and of no curvature, coarse elements will retain computing accuracy.

The first step of FEA work is to create a geometrical model. The model can be either built on the drawing board of the package or transferred from the model files from other software packages. We built the geometrical model directly on Ideas' drawing board. 2-D model is used due to its homogeneity in thickness. The parameter of the thickness was set for the model as an entity of element physical properties. Drawing the geometrical model, some curves had to be merged into 
compounded curves due to the limitation of maximum number of curves in one meshing area. Only one mesh area was produced due to the monolithic structure of the stage.

For automatic mesh generating, element sizing is a key step in which the parameters which determine the meshing size and size distribution are defined. The parameters have to be properly chosen to obtain the desired distribution of meshing density, more dense in curved and narrow areas and less dense in spacious areas to avoid consuming large computer memory space and processing time. The author set the global element size and length factor to be small in order to produce a high meshing density at hinge areas. The density at other spacious areas was reduced by setting large local element sizes. The percentage of curvature was chosen to increase the amount of element on curved edge. In the case of stage (S220), the parameters chosen for element size setting are listed in Table 4.5

\begin{tabular}{|c|c|c|}
\hline Global element size & $0.4 \mathrm{~mm}$ & \\
\hline & $10 \mathrm{~mm}$ & On outside edge of the frame \\
Local element size & $5 \mathrm{~mm}$ & $\begin{array}{c}\text { On inside edge of the frame } \\
\text { In spacious areas of levers and platform }\end{array}$ \\
\hline Percentage of curvature & 30 & \\
\hline Length factor & 0.4 & \\
\hline
\end{tabular}

Table 4.5: The parameters chosen for element size setting

The parabolic triangle element was chosen in the finite element modelling. Fig. 4.19 shows the meshed model which consists of 7134 elements and 15223 nodes.

\subsubsection{Boundary condition definition}

Boundary conditions which includes loads and restraints have to be applied to the model before it can be solved. To simulate the actual working condition, loads are added at each end of the blank spaces in which the PZT actuators are to be 


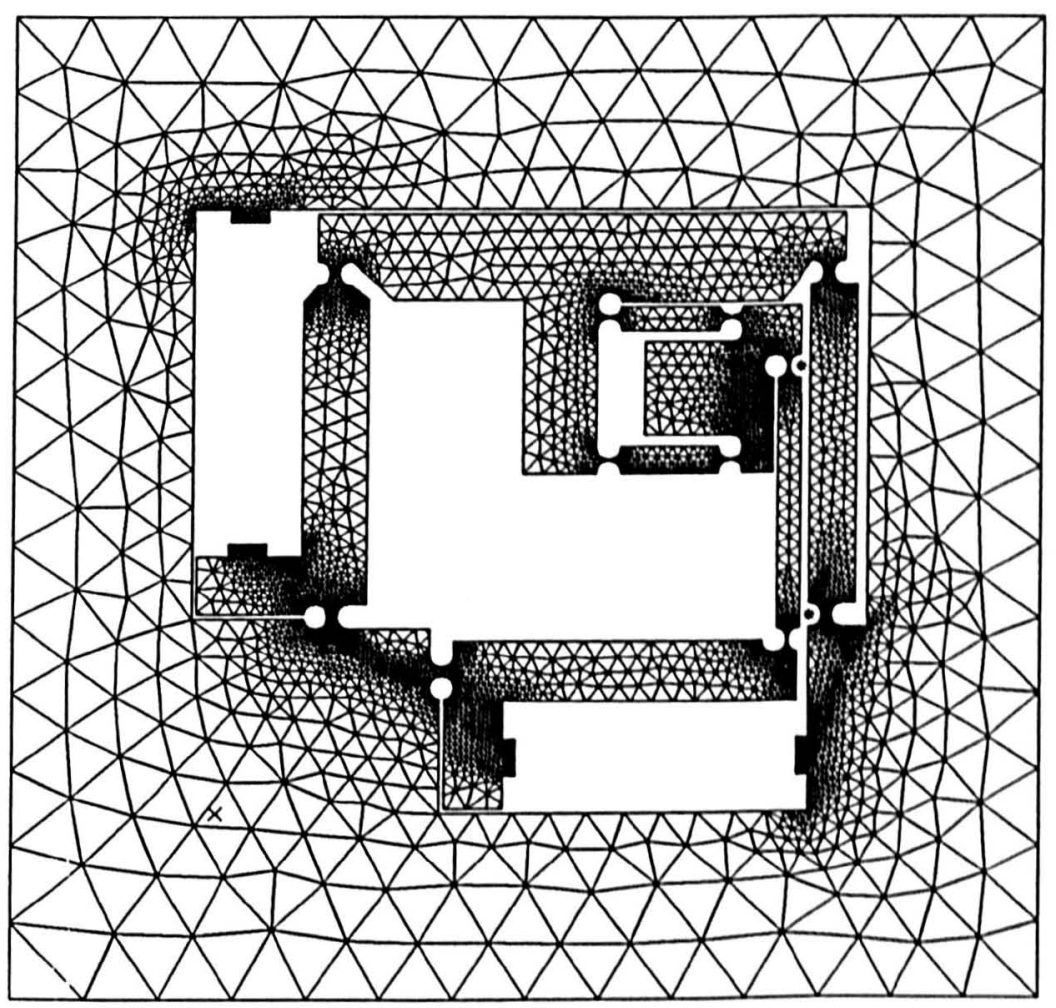

Figure 4.19: Diagram of meshed model for $x-y$ stage (S220) 
inserted, as shown in Fig. 4.20. Edge load of $10000 \mathrm{mN} / \mathrm{mm}$ is acted on four loading edges of $3 \mathrm{~mm}$ in length for each of them, leading to a total driving force of $30 \mathrm{~N}$ in each axis. Displacement restraints are applied to the stage to prevent the stage body moving and rotating freely, see Fig. 4.20. One of the restraints is applied to the node at the bottom-right corner on which motion is restricted in both the $x$ and $y$ axes. The other is put on the node at the upper-right corner with restriction only in the $x$ axis. In this way, all the mobilities of the stage body in the $x-y$ plane are restricted, however the freedom of its distortion is retained.

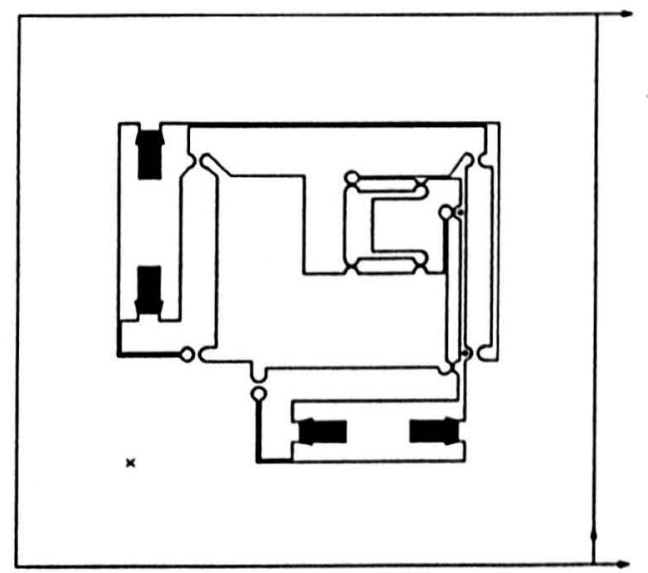

Figure 4.20: Diagram of boundary condition for FEA model

\subsubsection{Static deflections}

Fig. 4.21 shows computed deformation (solid line) with driving forces acted in the $x$ axis Fig. 4.21 (a) and in the $y$ axis Fig. 4.21 (b) respectively. The displacement of platform was obtained from the average displacement value of the element nodes on the platform. Then the system stiffness of the stage translation mechanism was calculated by the ratio of the driving force to the platform dis- 
placement. Table 4.6 gives the results of the static solution for two models. One (model-A) is the first prototype stage that was built and tested, and the other (model-B) is the modified model, both of which are similar in structure, but with different stiffness of hinges and levers.

\begin{tabular}{|l|c|c|c|c|}
\hline \multirow{2}{*}{} & \multicolumn{2}{|c|}{ model-A } & \multicolumn{2}{c|}{ model-B } \\
\cline { 2 - 5 } & $\mathrm{X}$ & $\mathrm{Y}$ & $\mathrm{X}$ & $\mathrm{Y}$ \\
\hline Driving forces $(N)$ & 30 & 30 & 30 & 30 \\
\hline Platform displacements $(\mu \mathrm{m})$ & 34.9 & 32.6 & 54.0 & 16.9 \\
\hline System stiffness $(N / \mu \mathrm{m})$ & 0.86 & 0.92 & 0.56 & 1.78 \\
\hline Yaw errors $(\mu \mathrm{r} d)$ & 234.3 & 176.7 & 6.9 & 0.19 \\
\cline { 2 - 5 }$(\mu \mathrm{rd} / 50 \mu \mathrm{m})$ & 335.7 & 271.0 & 6.4 & 0.6 \\
\hline
\end{tabular}

Table 4.6: The results of static solution of FEA

In Table1 4.6, the yaw errors of the stage model-A are much bigger than expected. The sources of the yaw errors can be analyzed from the distortion curves of local components of the stage. Fig. 4.22, 4.23, 4.24 and 4.25 are from the static solution of model-A. The component edges of $e-f, a-b, c-d$ and $j-k$ are labeled in Fig. 4.21 (a). The yaw error is obtained from Fig. 4.22, which gives the displacements (in the $y$ axis) of the nodes along platform edge $e-f$ against their coordinate locations when the stage is driven in the $x$ axis. Fig. 4.25 demonstrates the distortions of thick frame of the stage. Although the frame of the stage looks robust, it is still deformed by the driving force. From the figures we can see that the distortions are at sub-micrometer level, even though it is sometimes big enough to affect the performance of high precision stage. Fig. 4.23 and 4.24 show the distortion curves of $T$ lever on which the platform is supported. A large bending of the lever can be observed. This will directly result in the yaw of the platform and, therefore, was considered as a main source of the yaw errors.

Based on the analysis of the static solution given above, the design of the stage was modified. 'The modifications were done in mainly two aspects, increasing the 


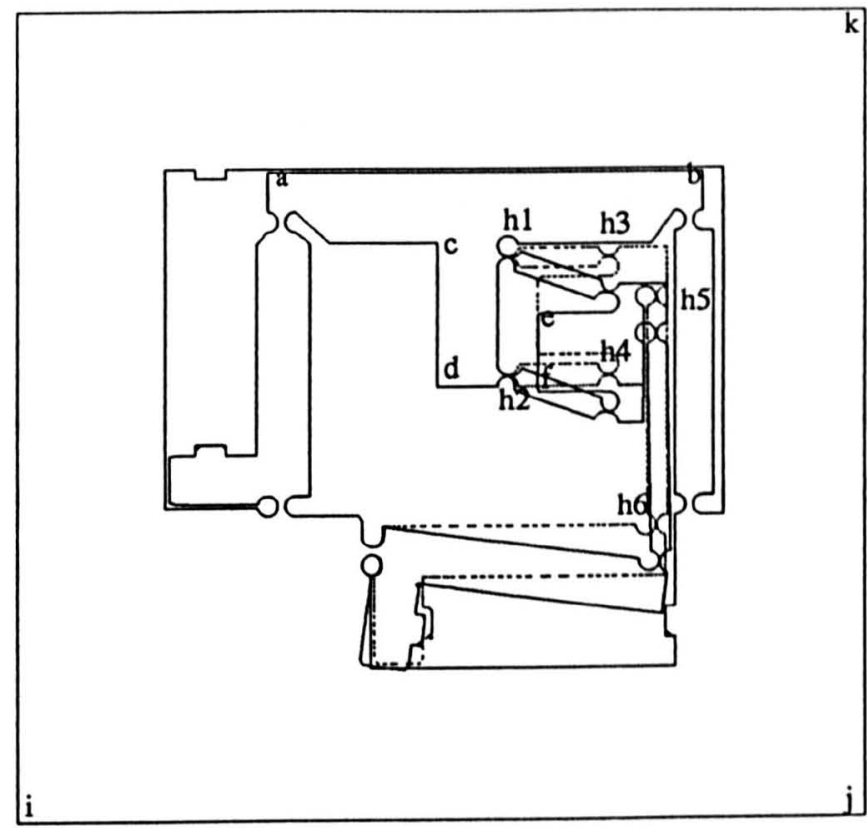

(a)

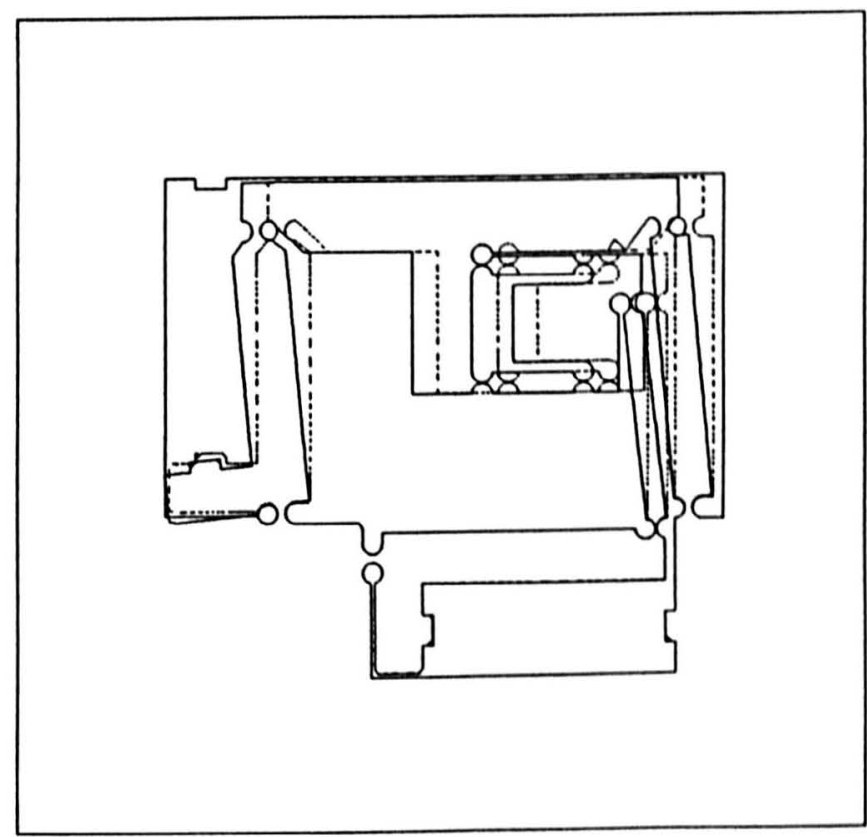

(b)

Figure 4.21: Deformed models from FEA static solution. (a) the stage is driven in the $x$ axis, (b) the stage is driven in the $y$ axis 
width of the levers, especially the $T$ lever $a-b, c-d$ in Fig. 4.21 (a), which was believed to be insufficiently stiff and reducing the stiffness of the hinges around the platform, $h 1, h 2, h 3, h 4, h 5$ and $h 6$, which produced large moments on the platform. With these in mind, model-B was designed. The width of $T$ lever section was changed from $4 \mathrm{~mm}$ to $6 \mathrm{~mm}$ and the hinge web thicknesses were reduced from $0.3 \mathrm{~mm}$ and $0.5 \mathrm{~mm}$ to $0.1 \mathrm{~mm}$ each. The results of static solution of the modified model are also given in Table 4.6 and correspondingly, Fig. 4.26, $4.27,4.28$ and 4.29 are the distortion curves. It is obvious that a significant improvement has been achieved. 


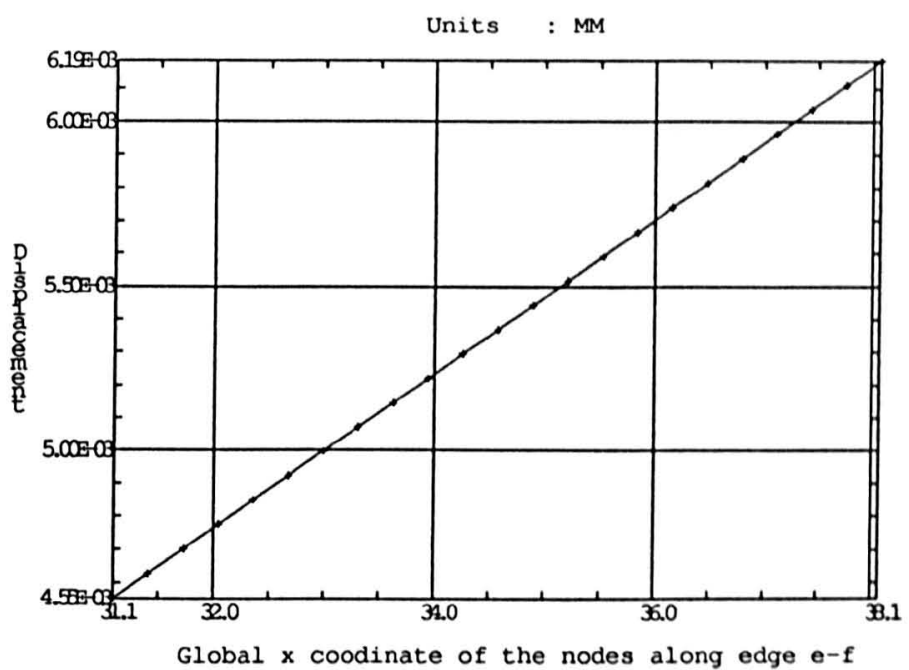

Figure 4.22: The yaw error of platform in the $x$ axis, (Model-A)

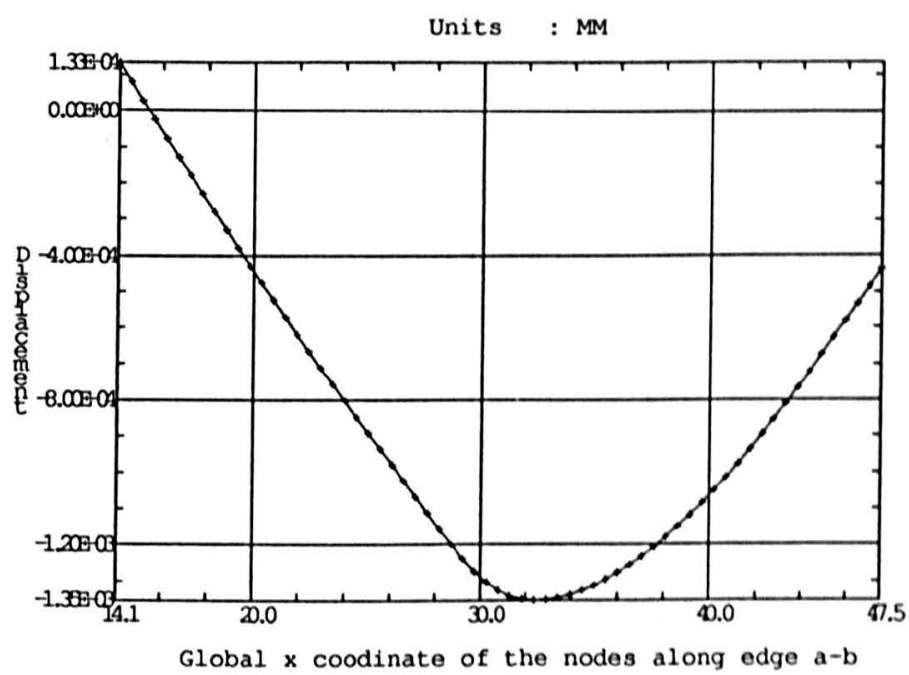

Figure 4.23: Distortion curve of nodes along $a-b$, (Model-A) 


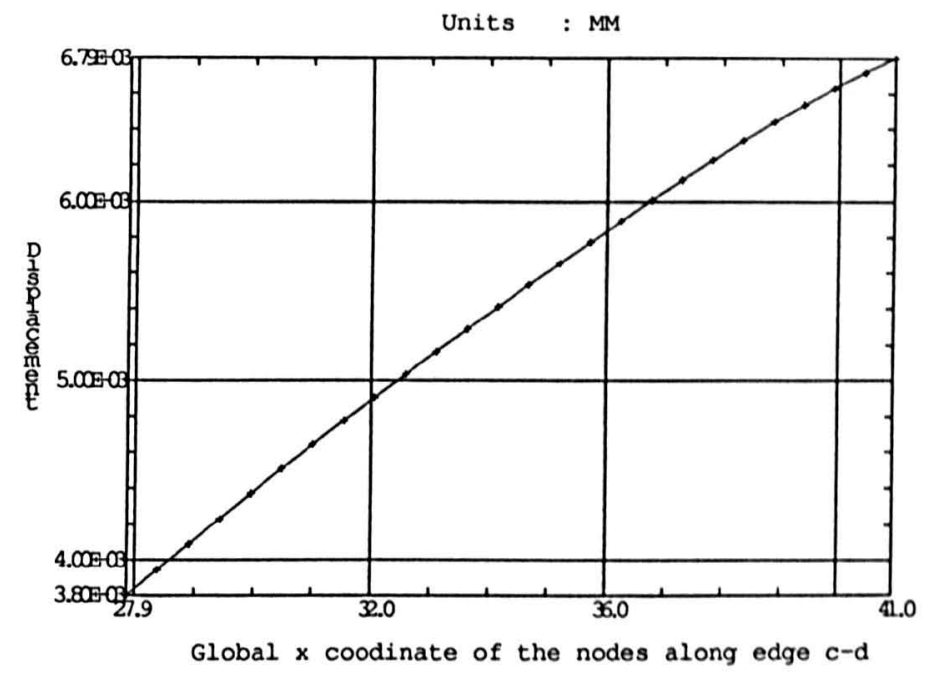

Figure 4.24: Distortion curve of nodes along $c-d$, (Model-A)

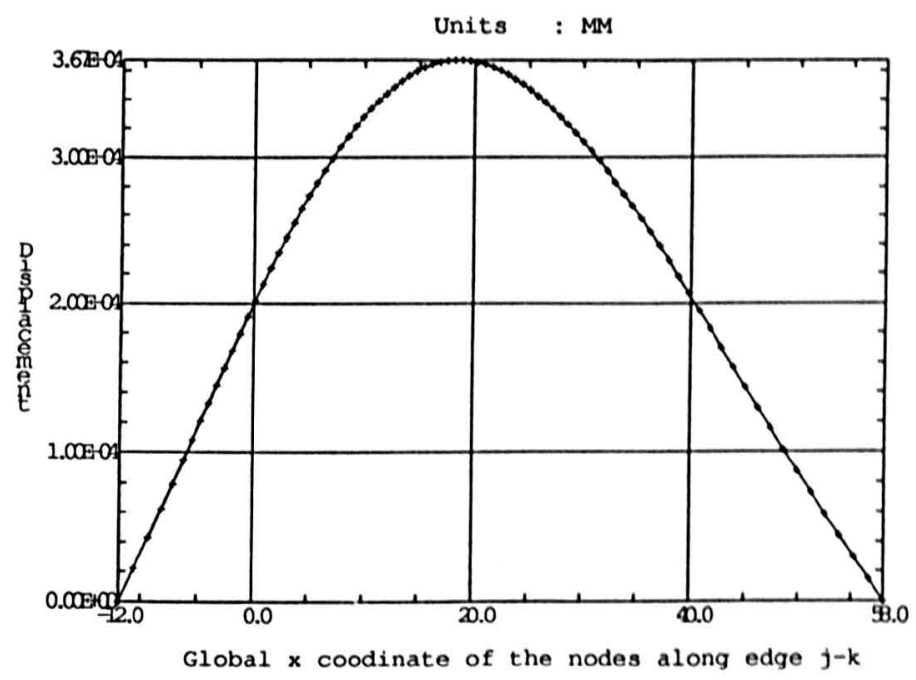

Figure 4.25: Distortion curve of nodes along $j-k$, (Model-A) 


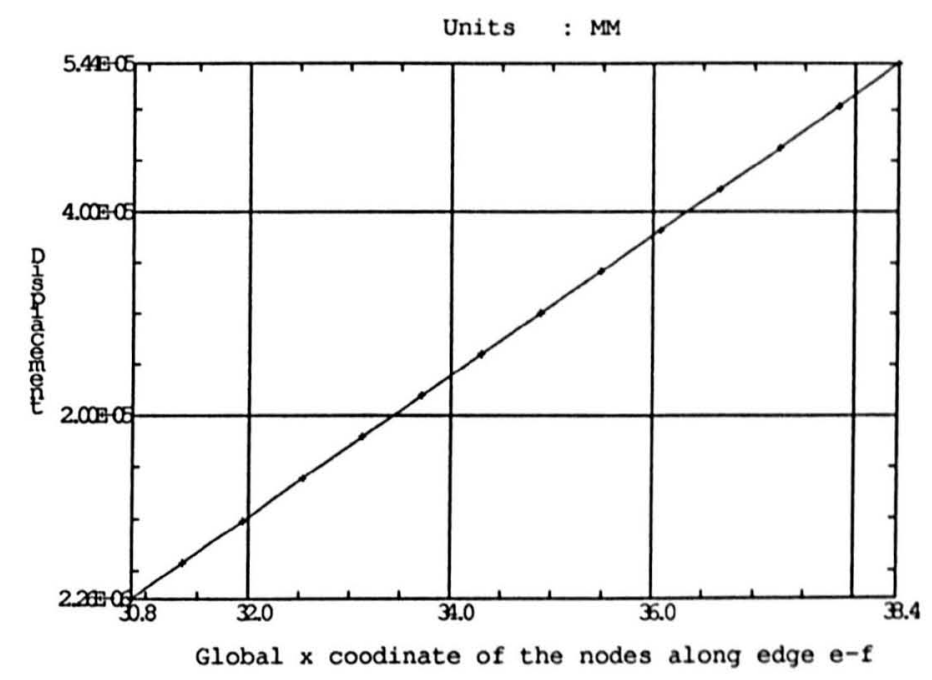

Figure 4.26: The yaw error of platform in the $x$ axis (Model-B)

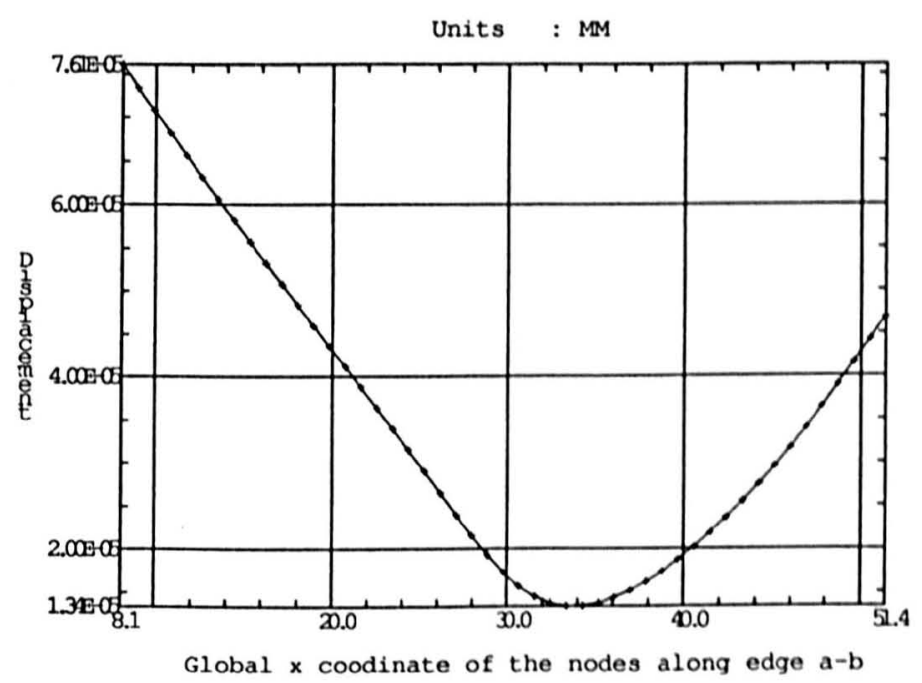

Figure 4.27: Distortion curve of nodes along $a-b$, (Model-B) 


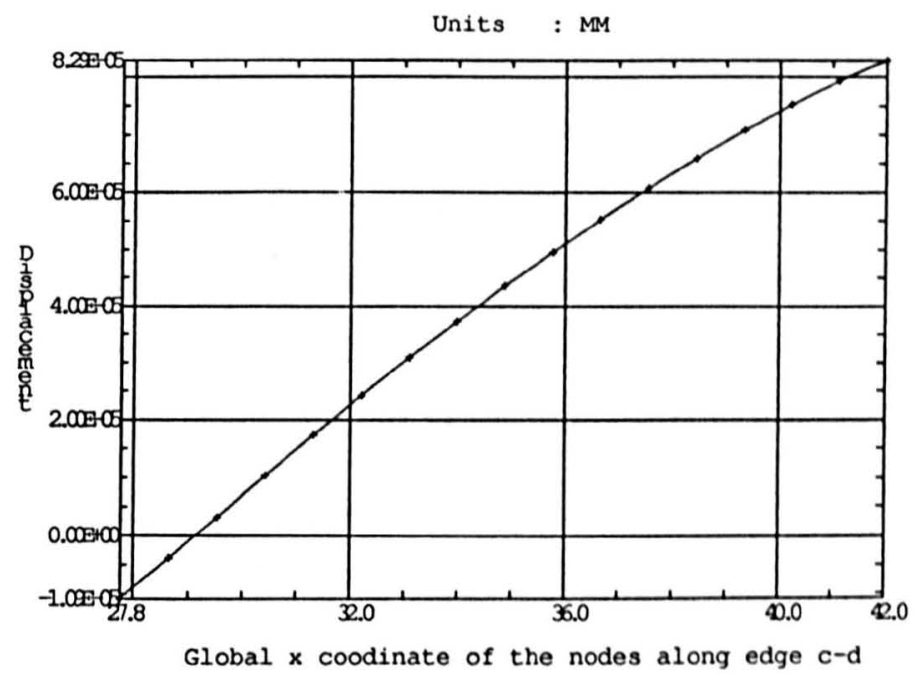

Figure 4.28: Distortion curve of nodes along $c-d$, (Model-B)

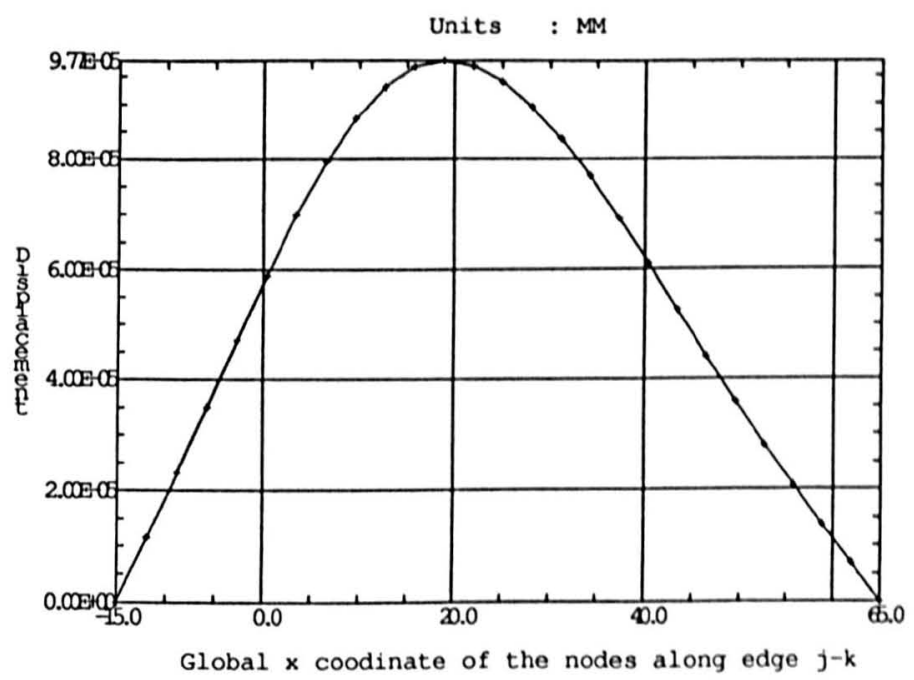

Figure 4.29: Distortion curve of nodes along $j-k$, (Model-B) 


\subsubsection{Dynamic solution}

The dynamic characteristics of the stage were also investigated with FEA models. Figure $4.30,4.31$ and 4.32 shows the three principal free vibration modes of the stage, the mode-1 corresponding to the lowest natural frequency, the mode2 to the second lowest natural frequency and the mode-3 to the next natural frequency. To include the dynamic enhancement effects of the piezo actuators and the preload springs, two spring elements were added in the stage model, fixing them on the levers at the driving point of the piezo actuators. The stiffness of the spring elements along their main deforming axes are set to be $8000 \mathrm{~N} / \mathrm{mm}$ and the other axes $100 \mathrm{~N} / \mathrm{mm}$. The results of the dynamic solution for model-B gave frequencies of $692 H_{z}, 1070 H_{z}$ and $2088 H_{z}$ for these three modes, respectively. From this it can be seen that the third frequency is much higher than the first and second, so the structure can be considered to be rigid in other directions except in the $x$ and $y$ axes.

From Fig. $4.30 \& 4.31$, it is clear that the $x$ and $y$ axes are the main vibration directions of the stage, corresponding to vibration modes 2 and 1 . The models we built for Lagrangian solution provide a similar solutions, in which we separated the spring translation system of the stage into two individual subsystems of single mobility based on the scanning directions of platform. 


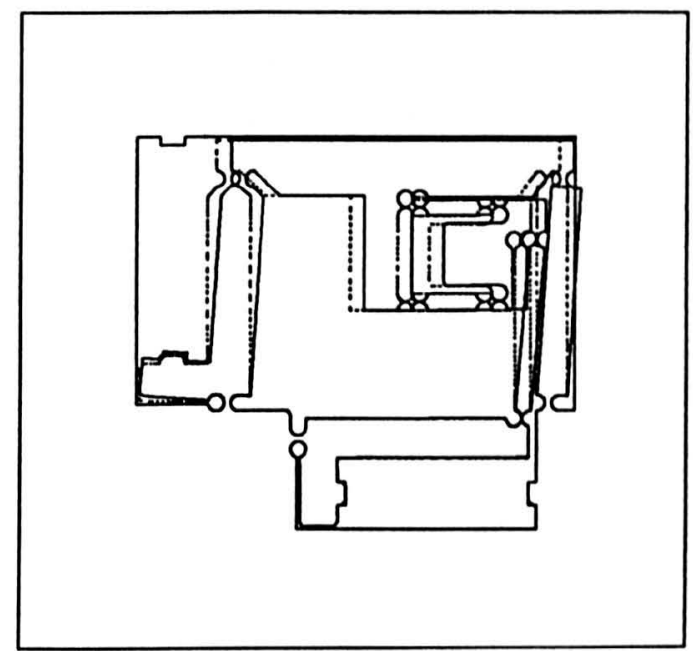

Figure 4.30: Free vibration mode of the stage, (mode-1)

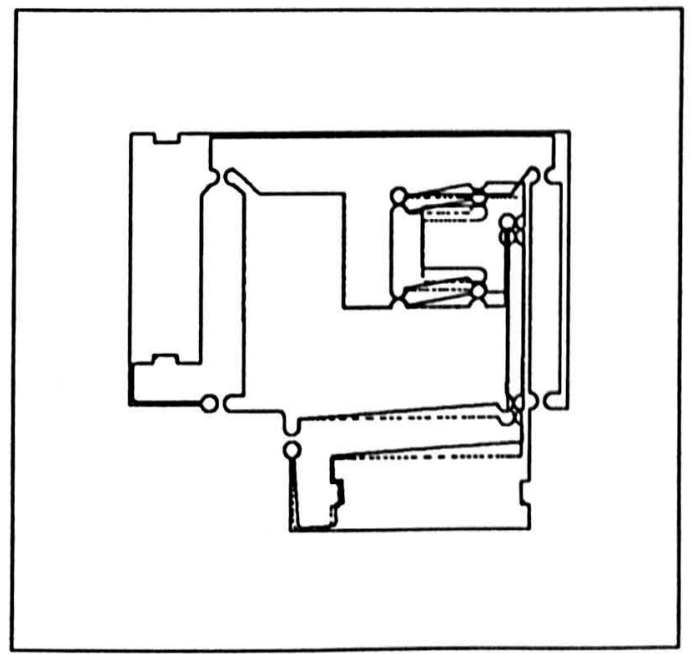

Figure 4.31: Free vibration mode of the stage, (mode-2) 


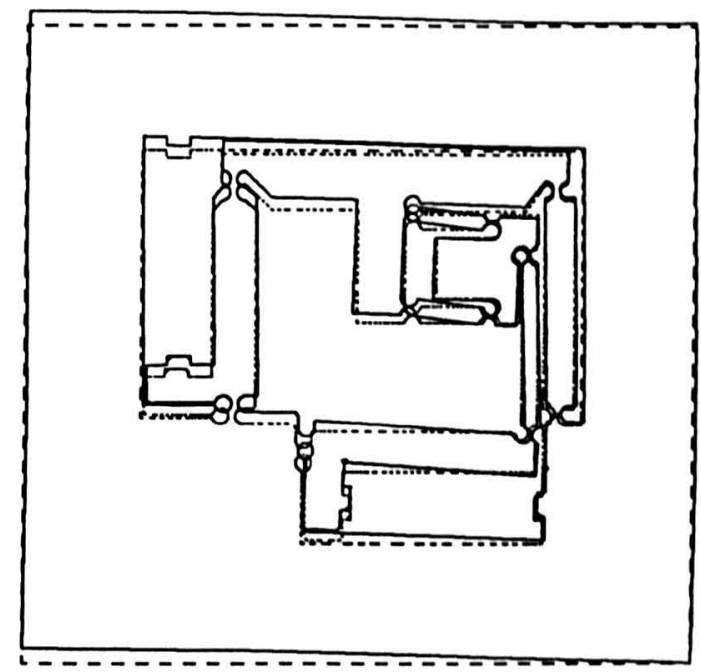

Figure 4.32: Free vibration mode of the stage, (mode-3)

\subsection{Discussion and conclusion}

Flexure translation mechanisms have been widely used in precision devices to provide ultra smooth and precise motions. Leaf spring and notch hinge spring are the most commonly used flexure components for the purpose. The capabilities of these two types of flexures are analyzed by comparing the performances of simple leaf spring rectilinear mechanism and simple notch hinge rectilinear mechanism.

The leaf spring system is more flexible to use when the deflection range is concerned. From equation (4.1) and (4.7), it can be seen that by keeping $d / L$ constant and increasing $L$ the deflection range of the leaf spring mechanism can be increased without losing system stiffness or by keeping $L^{2} / d$ constant and changing $d$ and $L$ the system stiffness can be adjusted to be higher or lower without affecting its maximum deflection ranges. However, for notch hinge mechanism of equations (4.2) and (4.11) the deflection range can be increased by increasing either $L$ or $R / t$, both of which will decrease the system stiffness. A certain stiffness is, sometimes, important for the stability and dynamic properties of a 
system. Moreover, the leaf springs store the elastic deflection energy in whole spring length so that the components are less strained. The notch hinges hold the energy in very small local area, resulting in a high strain in the components. The maximum principal stresses in deformed leaf spring and notch hinge spring mechanisms which have same stiffness and deflection are shown in Table 4.2. Therefore, from the points of view of deflection range and strain state, the leaf spring mechanism is superior to notch hinge mechanism.

In another aspect, the theoretical analysis indicates that simple notch hinge mechanism is more advantageous for high accuracy of performance, which, in most application cases of flexure mechanism, is the main concern of designers. From Table 4.1 and 4.2 it can be seen that the parasitic pitching errors caused by driving forces in leaf spring mechanism can be about twice as big as that in notch hinge mechanism. The errors can be reduced by properly designing parameters according to the equation 4.12 and 4.14. When the height of force acting line $L_{a}$ is moved to midway of spring leg, the pitching error is reduced to zero. Moreover, the notch hinge mechanism has higher buckling loads than leaf spring mechanism in the directions perpendicular to its translation axis. Usually, it is monolithically machined in a solid blank by CNC machine tool. The accuracy of the hinge flexure is primarily dependent upon the accuracy of the centers of the holes which form the hinges. Three sources of potentially significant errors have been investigated in this chapter. The results shows that the accuracy of performance of the mechanism is insensitive to manufacturing tolerances. This characteristic makes fabrication much easier.

The compound structures of rectilinear mechanism can be used to improve the accuracy of performance, and symmetric structure system provides more effective restrict to pitching of platform.

As an application case of flexure mechanism, the $x-y$ stage $(S 220)$ was designed and built. The experiment results of the first prototype of the stage showed that the errors of yaw, pitch and roll were larger than those that we expected. 
Then the stage was analyzed with FEA modelling, and large distortions in the translation mechanism were observed. A modified design of the stage shows a significant improvement in accuracy of performance. For design of flexure translation mechanism driven by piezo actuator, the piezo expansion can be amplified by the translation mechanism but the magnification has to match the stiffness of piezo element. 'The stiffness of the piezo element dominates the natural frequency of system. The amplification of its expansion will reduce the effective stiffness in square order. 'l'he loss of expansion range may also be a problem due to a high amplification of motion.

The FEA method is an effective tool for design of flexure translation mechanism. Using this technique, the performances of translation device can be predicted very well in both static and dynamic aspects. 


\section{Bibliography}

[1] R.V. Jones, 1962, Some uses of elasticity in instrument design, J. Sci. Instrum., 39,, $193-209$

[2] P.H. Sydenham, 1984, Elastic design of fine mechanism in instruments, $J$. Phys. E: Sci. Instrum., 17, 922 - 930

[3] F.E. Scire and E.C. Teague, 1978, Piezodriven 50- $\mu m$ range stage with subnanometer resolution, Rev. Sci. Instrum., 49 (12) 1735 - 1740

[4] S.T.Smith and D.G.Chetwynd, Foundations of Ultraprecision Mechanism Design, Gordon and Breach Science Publishers, London, 1992

[5] R.V. Jones, 1951, Parallel and rectilinear spring movements, J. Sci. Instrum., 28, 38 - 41

[6] S.T.Smith, D.G. Chetwynd, and D.K. Bowen, 1987, Design and assessment of monolithic high precision translation mechanisms, J. Phys. E: Sci. Instrum., 20, 977 - 989

[7] S.T.Smith, D.G. Chetwynd, and S. Harb, 1993, A simple two-axis ultraprecision actuator, Rev. Sci. Instrum., 65 (4) $910-917$

[8] J.M. Paros and L. Weisbord, 1965, How to Design Flexure Hinges, Machine design, November 25, $151-156$

[9] R.V. Jones, 1956, Some parasitic deflexions in parallel spring movements, $J$. Sci. Instrum., 33, $11-15$ 
[10] Private communication with Queensgate Instruments Ltd.

[11] E.C. Teague, R.D. Young, F. Scire, and D. Gilsinn, 1988, Para-flex stage for microtopographic mapping, Rev. Sci. Instrum., 59 (1) 67 - 73

[12] T.H. McWaid and J. Schneir, 1994, The Development of a Calibrated Atomic Force Microscope, Proceedings of ASPE 1994 Spring Topical Meeting. 


\section{Chapter 5}

\section{Metrological SFM system}

\subsection{Introduction}

Both scanning tunneling microscope (STM) and scanning force microscope (SFM) are capable of a nanometer or subnanometer lateral and vertical resolution. Many of this generation SPMs have successfully achieved the ability of producing surface topographical images at atomic levels, which approaches the physical limits of resolution for this surface measurement technique. However, as the applications of SPM are spread to more and more industrial fields, the requirements of scanning probe microscopies have been extended from high resolution to high accuracy. The new generation of SPM should be able to not only produce topographic images of specimens with nanometer or subnanometer resolution, but also to work as a metrological measurement tool with the accuracy at this level or to do microfabrication with the same precision level.

At present, almost all of the SPMs use piezoelectric components as translators for $x-y$ scanning and $z$ profiling. Commonly, translators are in the form of tube, tripod, or bimorph scanner. A big problem of using these scanners is their inherent nonlinearity which is mainly caused by the nonlinear response to the applied voltage, hysteresis and creep of piezoelectric elements (PZT) and the coupling between the motions of $x, y$ and $z$ axes. Because of this, distortions always exist 
in the topographic image from these scanning techniques. Sometimes, over $40 \%$ difference in image scales from one region of the image to another can result [4].

Many strategies have been reported to reduce these errors. One way is to linearize the expansion of piezoelectric actuator by providing nonlinear compensatory drive voltage using a predetermined algorithm [3]. Alternatively a charge drive can be used [5][6]. With these, the nonlinear errors can be reduced to certain extent but, at present, not adequately for many applications. A more satisfactory method is to directly monitor the tip or specimen position with high sensitivity transducers to eliminate the errors in the ways by the use of either post-scan image correction or closed loop feedback control [4][7] - [11]. With this method, the nonlinear response, hysteresis and creep can be reduced to insignificant levels.

Our metrological scanning force microscope (SFM) is developed based on the position monitoring strategy with the objective of achieving both high resolution and accuracy at nanometer or subnanometer levels. The instrument should be capable of conducting routine measurement of precision engineering surfaces at this level. In this chapter, the principle of operation of the metrological SFM is introduced. Integration of the various sub-systems into a working instrument is then described, which includes discussions of the force probe and vertical DPT translator system, $x-y$ scanning stage, coarse positioning and approaching mechanism, and instrument base. A brief introduction on electronic specifications and computer software is included. The characteristics of the instrument system are experimentally assessed and analyzed. Finally, various specimens from a wide range of engineering areas have been measured using this SFM to illustrate its metrological capability.

\subsection{Principle of operation}

The metrological scanning force microscope of this study mainly consists of a probe and its vertical translating system, a precision $x-y$ scanning stage, a coarse 
positioning mechanism, and electronics. Fig. 5.1 is a schematic diagram of the instrument showing modular components of the system and Fig. 5.2 a photograph of the system. The specimen is held on the scanning platform of the $x-y$ scanning stage (S220), in which piezoelectric actuators are used for the drive mechanism with a lever ratio of four to one giving a range of $50 \mu \mathrm{m}$ and a natural frequency of above $500 \mathrm{H}_{z}$ in both axes. Two capacitance sensors, Queensgate Instruments NanoSensor ${ }^{\mathrm{TM}}$, are built in the stage for in-situ monitoring of platform position. The NanoSensor ${ }^{\mathrm{TM}}$ is capable of resolving better than $0.1 \mathrm{~nm}$ in a range of hundreds of micrometers with a frequency response of up to $5 k H_{z}$, and its linearity error is less then $0.2 \%$. The outputs of the sensors are used as feedback for closed-loop control resulting in a very low crosstalk and nonlinearity. The scanning of the X-Y stage is controlled from a PC computer through a servo module and PZT driver for each axis. The design details of the stage are discussed in Chapter 4.

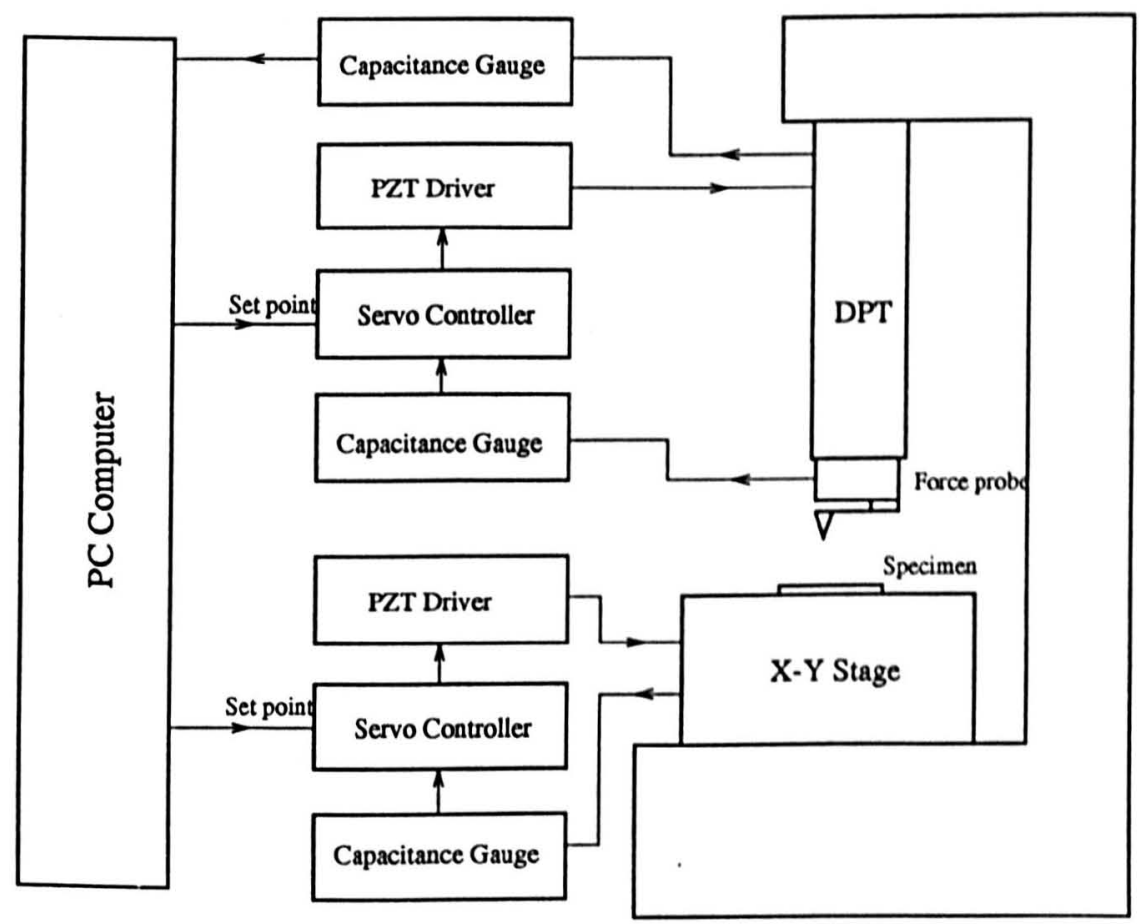

Figure 5.1: Block diagram of SFM system 


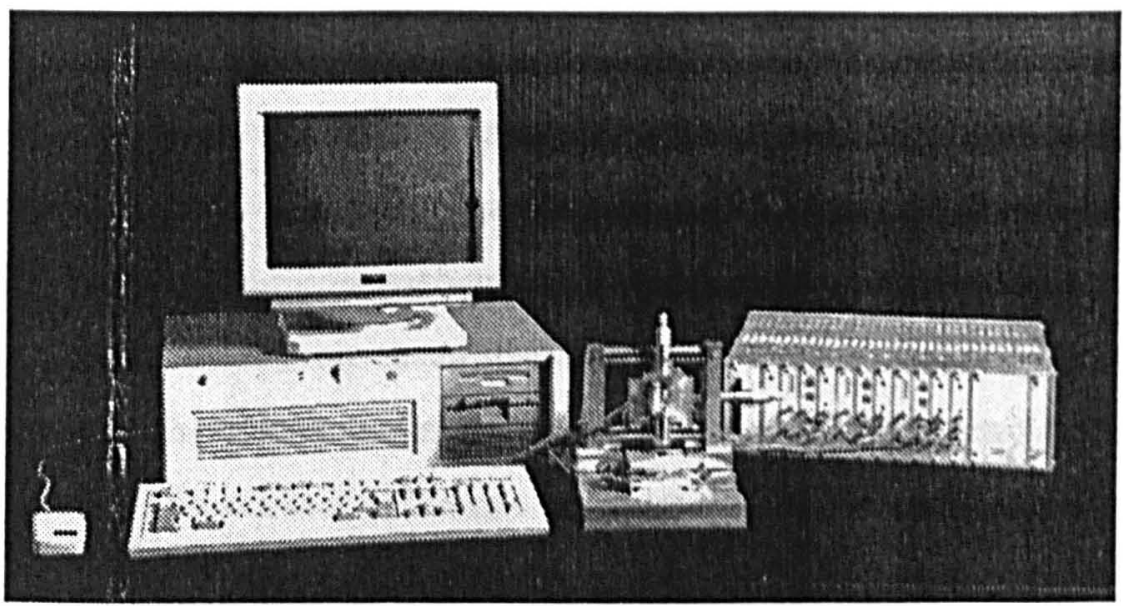

Figure 5.2: Photograph of the SFM system

Three dimensional imaging is achieved by positioning a force probe above the specimen, orthogonal to the scanning plane of the $x-y$ stage. The force probe, a capacitance force sensor, consists of a stylus type diamond tip attached on a small cantilever beam with capacitance electrodes on the rear face being used to sense any distortion caused by the interaction between tip and surface. For the details of the force probe see Chapter 3. The force probe is mounted on a linear translator, Queensgate Instruments Digital Piezoelectric Translator (DPT), in the vertical, or $z$, axis. The translator consists of a piezoelectric actuator with a capacitance sensor along the central axis to monitor the actual expansion. The output from the force sensor is maintained at constant value again by feedback through a PID controller to the vertical translator, i.e. the contact force between tip and specimen is kept constant by the feedback closed loop control. While the $x-y$ stage is scanning, the force probe driven by the vertical translator will follow the contour of constant force which is interpreted as the mechanical surface height. For traditional scanning force microscopes, the expansions of $z$ translators are assumed to be of linear relationship with driving voltages which are used as the output of measurement. In these cases, nonlinear errors in the measurements are inevitable. When using the DPT, the 
measurement results come from the output of its integral capacitance sensor which therefore measures the actual expansion of PZT element, eliminating the undesirable nonlinear characteristics. The DPT translator has a linear expansion range of $15 \mu \mathrm{m}$ with subnanometre resolution and a working frequency bandwidth of $5 \mathrm{kHz}_{\mathrm{z}}$. When calibrated with $\mathrm{x}$-ray interferometry, the capacitance gauges have been shown to exhibit accuracies of better then a tenth of a nanometer [12]. Because of their hardness and well defined geometry diamond tips are used for the probe. The contact force can be controlled over a wide range from 10 ${ }^{-5}$ to $10^{-8} N$ with this kind of probe, covering the entire force range generally encountered in both atomic force microscopes and precision stylus profilers.

Fig. 5.3 shows a line diagram of the mechanical system of the SFM. The $x-y$ scanning stage is fixed on a $25 \mathrm{~mm}$ thick aluminium base. The $z$ translator on which the force probe is attached is, in turn, mounted on a three axis coarse positioning device. Three mechanical micrometers are used as positioning actuators providing a coarse adjustment range of $25 \mathrm{~mm}$ in $x, y$, and $z$ axes with resolution of approximately $10 \mu \mathrm{m}$. The specimen scanning and data acquisition for the microscope is controlled by a PC computer using an in-house software package providing stage positioning, data sampling and image processing under a Windows environment.

Before the surface can be measured, the specimen and probe have to be carefully set up. The specimen is first fixed on a sample holder with double sided stick tape loaded onto the scanning platform of $x-y$ stage. The probe system is then manually moved down towards the surface using a vertical coarse approach micrometer. The forces from the micrometer are transferred through a bysteretic coupling to drive the vertical translator and probe system until the tip is in contact with the surface. To make full use of the translator, the actuator of the DPT should be set in the middle of its expansion range. After the probe and actuator have been positioned, it is possible to retract the micrometer so that it no longer forms part of the measurement loop. 
For the consideration of vibration, the instrument is mounted on a general metrology table consisting of a heavy granite plate $(1 \mathrm{~m} \times 2 \mathrm{~m} \times 0.15 \mathrm{~m})$ supported by a steel frame of six leveling feet with $10 \mathrm{~mm}$ thick rubber pads underneath to isolate high frequency vibration. No obvious effects from ground and airborne vibration have been found in our experiments.

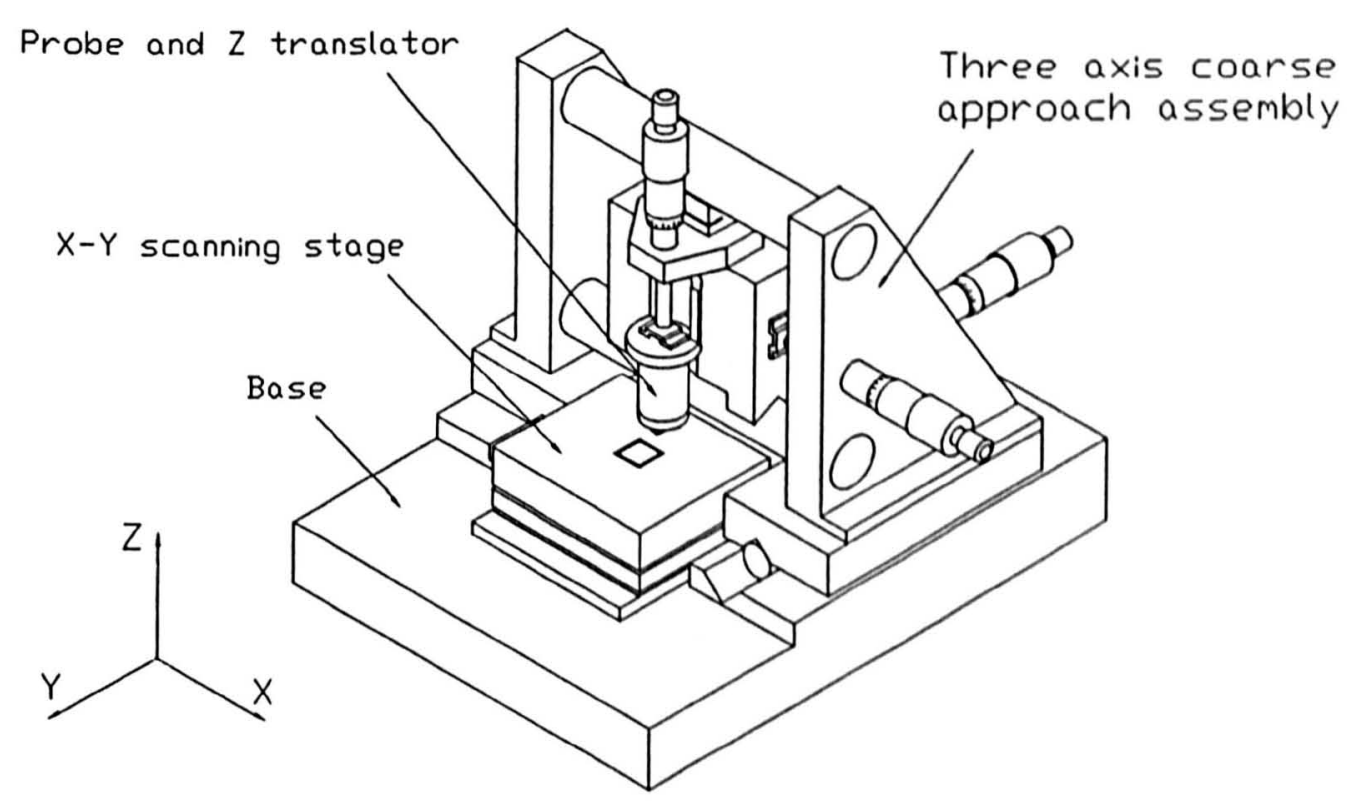

Figure 5.3: Isometric sketch of the metrological SFM

\subsection{Some design details}

\subsection{1 $\mathrm{X}-\mathrm{Y}$ axis coarse positioning mechanism}

Fig. 5.4 shows a diagram of the $x-y$ axis coarse positioning mechanism. A parallel slideway, along the longitudinal, or $y$, axis, is machined into the base. Upon 
this slideway rides two axis coarse positioning assembly. This $y$ axis slideway is composed of a pair of vee and flat grooves on both opposite surfaces between the base block and the bottom of the assembly, separated by a pair of polished brass rods. A coarse actuator (MY), a mechanical micrometer, is fixed on the base and provides driving forces through a hysteretic coupling to the coarse positioning assembly to drive it in the $y$ axis. A locking mechanism is applied to the

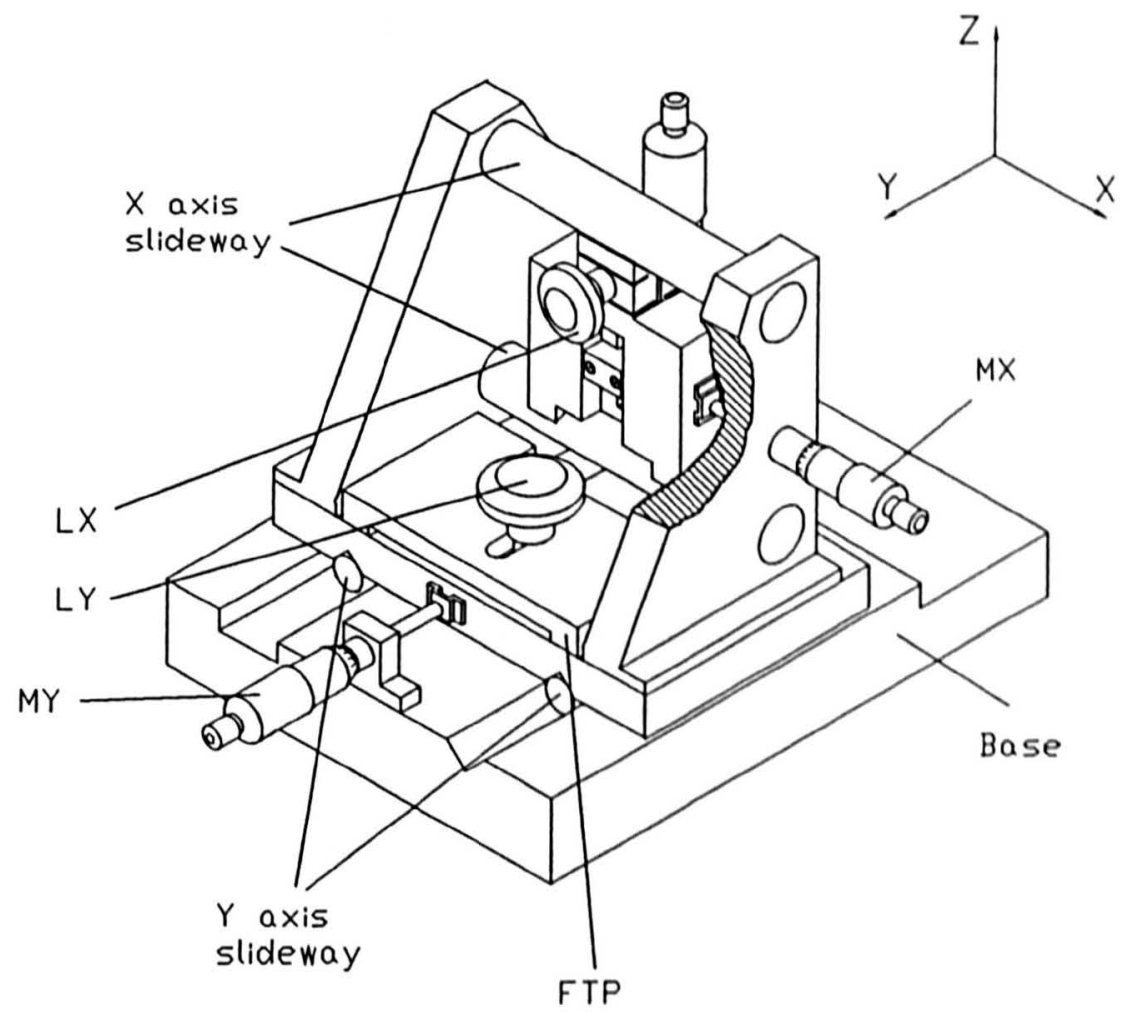

Figure 5.4: Isometric sketch of the X-Y coarse positioning mechanism

slideway. A cross section view of the locking mechanism is shown in Fig. 5.5. An $M 8$ locking bolt (LY) passes through slots in the force translator plate and the moving substructure and screws into the base block. When locked, the bolt's shoulder supplies a clamping force through the force transferring component (FT) to the base plate through the slideway rods. The force transferring component 


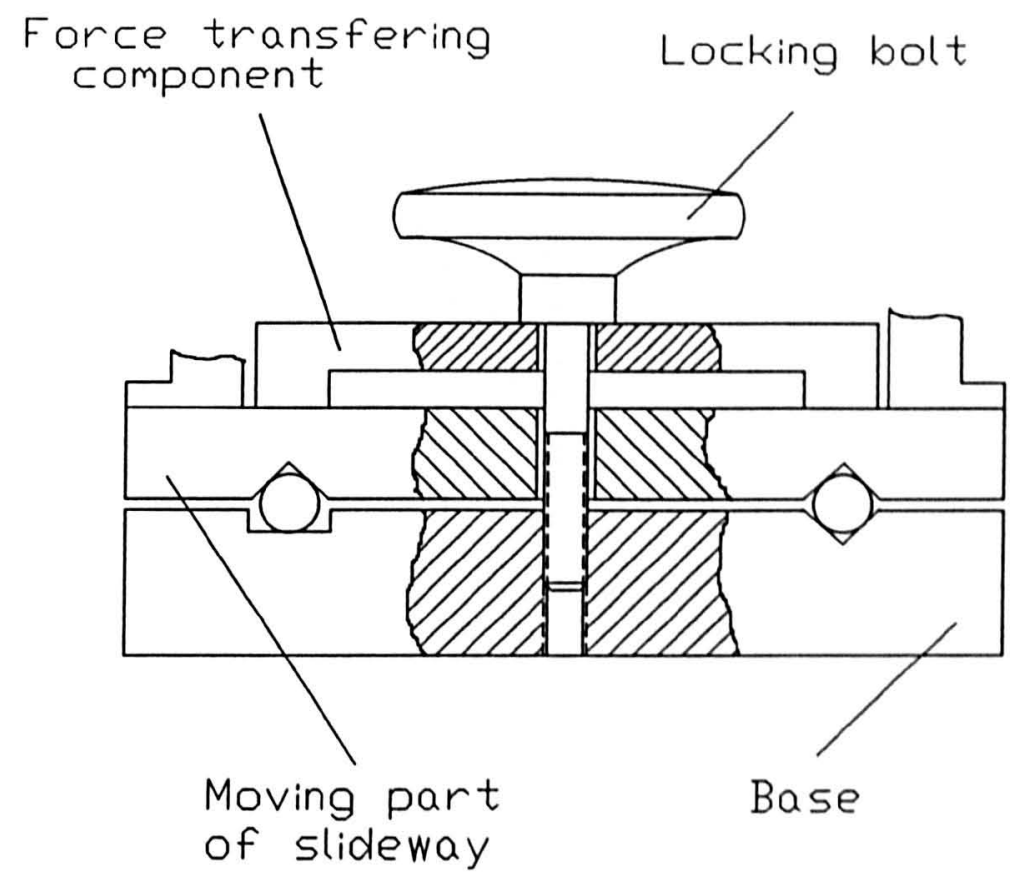

Figure 5.5: Section view of $y$ axis locking mechanism

contacts the bottom plate with four small plateaus cut onto its bottom surface of the component at four corners. The contact spots are on the parallel axial lines of the slideway, ensuring that the locking force will produce no bending moment on the plate, and thus generate a very small elastic distortions in the system. Above these in Fig. 5.4 is a micrometer (MX) acting on the $z$ translator carriage through a hysteretic coupling. The carriage rides on two parallel solid steel bars supported on thick plates at each end. These two steel bars are $25 \mathrm{~mm}$ in diameter giving a very high stiffness in the $z$ axis. The carriage holding the $z$ axis translator is machined from an aluminium block. At the top and bottom of this block vee grooves contact the steel rods to form a slideway. This slideway is perpendicular to the slideway on the base block, thus providing the second axis drive for $x-y$ positioning. To ensure constant contact of this $x$-axis slideway, the top part of the block is necked with two parallel narrow slots cut deep into the block in both front and back surfaces and a vertical narrow slots cut into the 


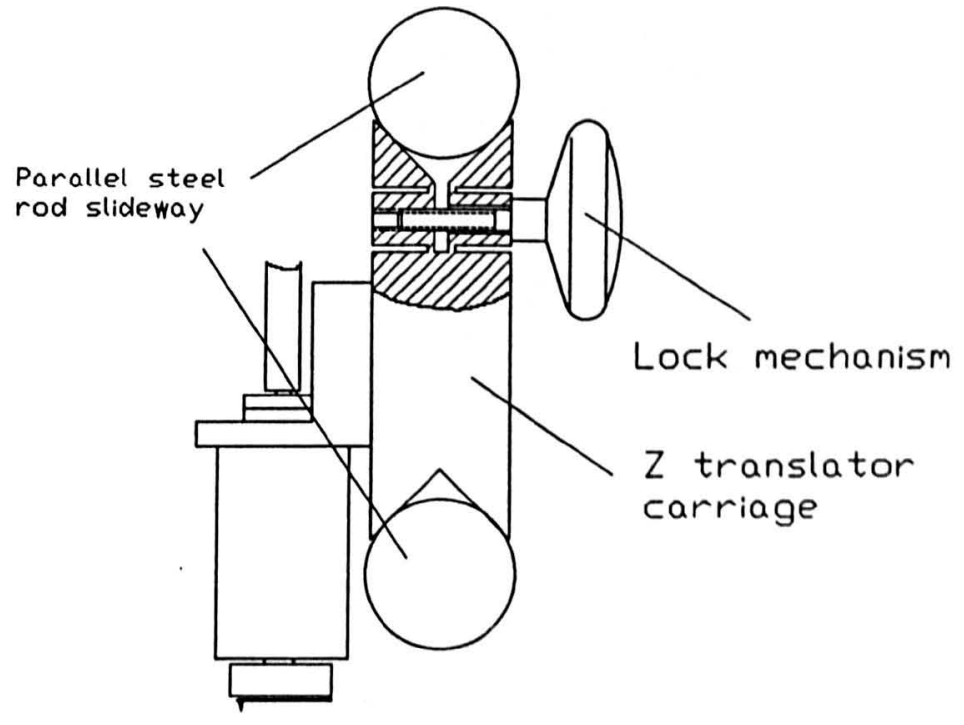

Figure 5.6: Diagram of elastic contact mechanism of $Z$ translator carriage

block along the bottom of the top vee groove to provide an elastic compliance, see Fig. 5.6. When a clamp force is properly applied against the block face by a locking bolt (LX), the clearance in the top slideway will be eliminated and the elastic contact guarantees the carriage moving along the slideway smoothly. The assembly can be locked in both the $x$ and $y$ axes leading to an increase in stiffness. Again the micrometers are connected with hysteretic couplings so that they can be decoupled after coarse positioning.

\subsubsection{Probe coarse approach mechanism}

A vertical vee groove is cut into the front face of the $\mathrm{x}$-axis carriage to guide a DPT holder in the $z$ axis. The DPT holder is secured in the vee groove by a spring assembly acting from the back of the carriage, pulling the holder against the carriage with its curved surface, see Fig. 5.7. The spring is adjusted so 

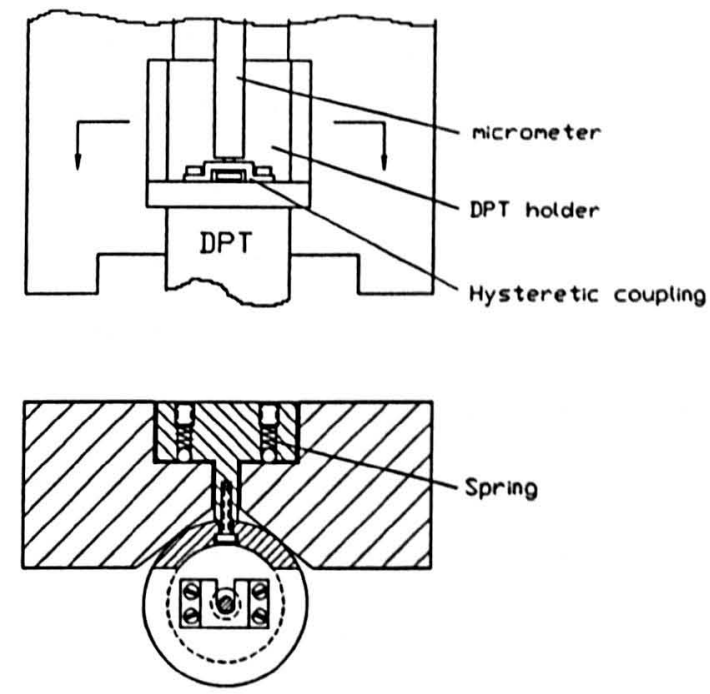

Figure 5.7: Diagram of decoupling mechanism

that the static friction force is sufficient to prevent the DPT holder sliding down under its own weight, but low enough to enable sliding on application of a reasonable force from the micrometer. The DPT thus remains in position after coarse approach micrometer retracts clear from the measurement loop through the hysteretic coupling.

\subsubsection{Measurement loop and material selection}

For the first prototype of this metrological SFM, the base, frame and most of the other components except $z$ transducer and slideway rods are made of aluminium alloy because of its ease of manufacturing and favorable mechanical and thermal properties. Thermal effects are always a main concern for material selection in the design of precision instruments. Basically, there are two approaches to the problem: The use of low thermal expansion materials such as Zerodur ${ }^{\mathrm{TM}}$, fused silica and Invar ${ }^{\mathrm{TM}}$, where the instrument is geometrically and dimensionally less susceptible to environmental temperature change. However, these materials usually have poor machinability and are relatively expensive. The other competing 
choice is to use inexpensive and easy to machine materials which usually have high thermal diffusivity. With that, the effects of thermal shock resulting from manual operation or some other local thermal contacts can be rapidly dissipated and therefore the settling times reduced. However, it makes instrument very susceptible to temperature change and so the instrument often has to be used in a temperature controlled environment.

As shown in Fig. 5.8, the instrument measurement loop goes down from the $x-y$ stage to the base. Then it passes upwards through the coarse positioning assembly and the $z$ transducer carriage and finally through the transducer itself to the force probe. The ideal structure should be designed in a way that all components in the measurement loop have equal expansion coefficients and the same thermal capacities, then thermal expansions can be compensated itself in the loop and, ideally, a zero expansion is possible. A symmetric structure can be adopted to reduce the effects of lateral thermal expansion. However, the actual situation of our instrument departs somewhat from the ideal case, so that the thermal drift in the measuring process is inevitable.

Theoretically, the fewer the components involved in the measurement loop, the less the unstable error sources will be. The mechanical micrometers for coarse positioning in the $x$ and $y$ axes and coarse approach in the $z$ axis are decoupled from the measurement loop after the probe is properly positioned. This can avoid the possible creep caused by residual strains at the interface between the couplings and the micrometers, which result from the driving forces. Therefore it is found to be very effective for the improvement of system stability.

\subsubsection{Electronic specification}

All the electronics used in our metrological SFM are modularized according to their functions as shown in Fig. 5.9 in which each block corresponds to an electronic module. All of these modules are assembled into a Queensgate Instruments S2000 rack, combining as a S2000 control system. The system consists of four 


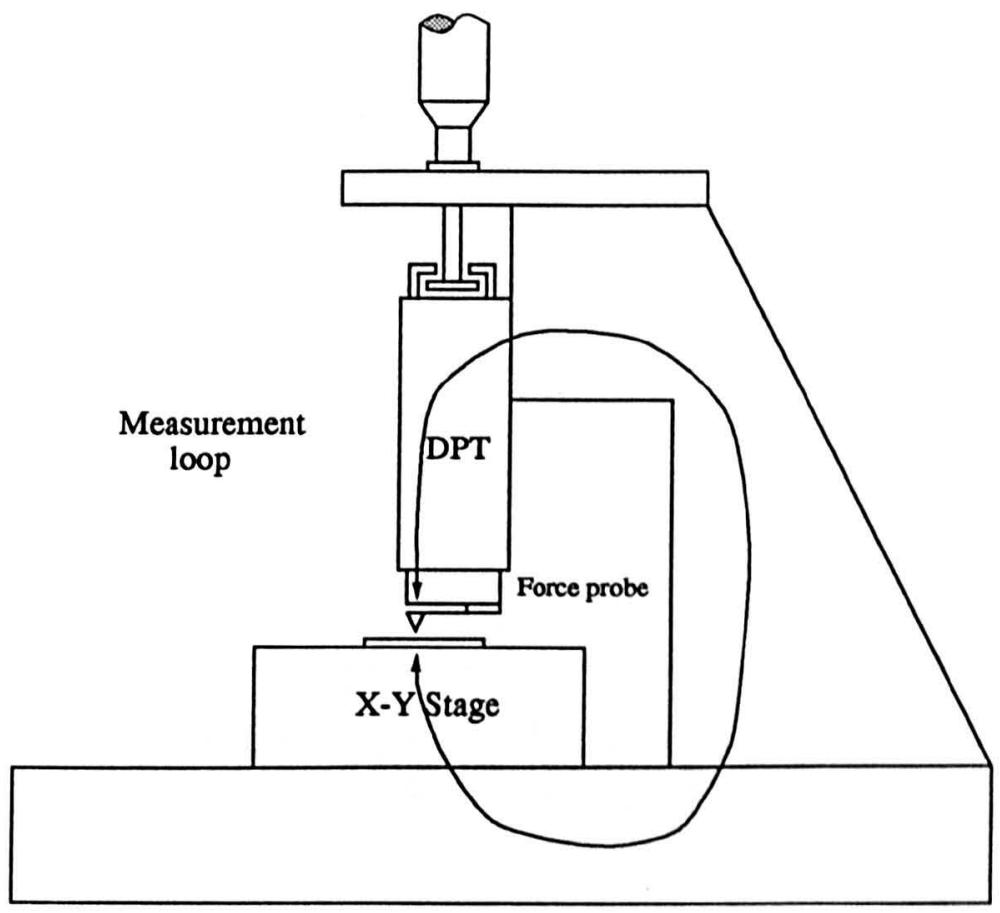

Figure 5.8: Schematic diagram of the measurement loop of the instrument

NanoSensor modules for modulations of the signals from the capacitance sensors integrated in the $x-y$ stage, $z$ translator DPT and from the capacitance based force probe. Three SM Servo Modules and DM Driving Modules conduct controlling and driving to the PZT actuators in three axes. An OSO Oscillator Modul provides a $30 \mathrm{kH}_{z}$ drive voltage and $4.5 \mathrm{~V}$ dc reference for all the NS NanoSensor Modules in the S2000 rack. A National Instruments PC-DIO-96 card is used to match the PAR parallel interface of the system that allows the S2000 system to be controlled by a computer via a high speed parallel I/O bus.

Each NanoSensor module has a 16 bit A/D converter chip, so that both analogue and digital outputs are provided. The calibration, which will be described later, gives a resolution of $0.216 \mathrm{~nm} / \mathrm{bit}$ for the outputs of NanoSensor module of the $z$ translator and $0.88 \mathrm{~nm} / \mathrm{bit}$ for that of the $x-y$ stage. The linearities of the modules are calibrated to be better than $0.2 \%$ by manufacturer and noise level $<0.2 n m H_{z}^{-\frac{1}{2}}$ (RMS) when working with the NanoSensor we used. The 


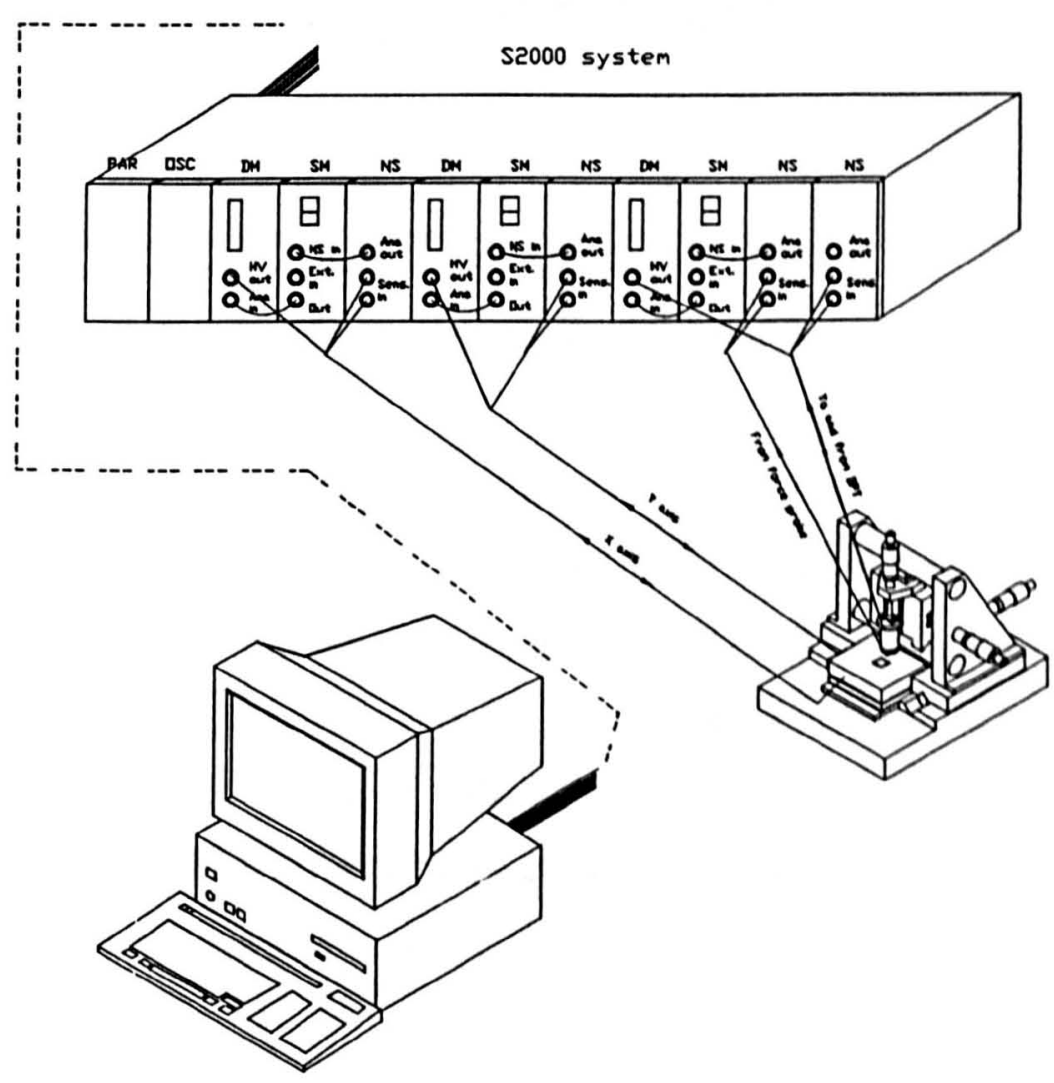

Figure 5.9: Schematic diagram of S2000 rack system 
Servo module generates a command voltage corresponding to its reference which can be controlled by either an external analogue input, a digital input or a signal generated from a up/down counter which is adjusted manually by the front panel push buttons. This command is then compared with the NanoSensor output and the difference signal is amplified and integrated, see Fig. 5.10. The resultant

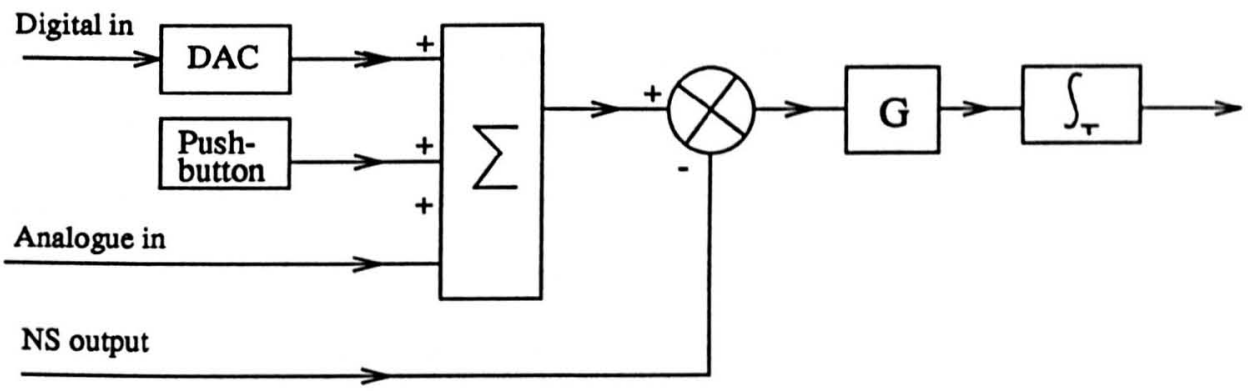

Figure 5.10: Block diagram of of SM Servo Module

outputs are sent to a DM module. In our case, only digital input is used. The Servo Module has two 14 bit D to A converters, fine and coarse. The coarse DAC allows a control over full range of translator $\pm R / 2(R=$ full range $)$ and the fine $\mathrm{DAC}$ gives a precision control over a limited range of $\pm \mathrm{R} / 16$ with a resolution of $0.425 \mathrm{~nm} /$ bit for the $x-y$ stage. DM Driving Module is a high voltage amplifier which is optimized for driving PZT actuator. It is controlled by a Servo Module and supplies a voltage in the range of $0 \mathrm{~V}$ to $+175 \mathrm{~V}$ to the PZT actuator.

\subsection{Performance evaluation}

\subsubsection{Instrument calibration}

Before an instrument can be used for metrological measurement, it has to be calibrated. Although the NanoSensors ${ }^{\mathrm{TM}}$ we used in the $x-y$ scanning stage and DPT had been calibrated by manufacturer, it is still necessary to calibrate the instrument system after integration. The $x-y$ scanning stage was calibrated using a Hewlett Packward 5505A laser interferometer system. Fig. 5.11 is a schematic 
diagram of the calibration system. A collimated laser beam goes through a polarized beam splitter and is separated into two beams. One of them travels straight ahead and is reflected by a mirror which is settled on the center of scanning platform of the stage with its reflecting face perpendicular to the moving axis to be calibrated and the other beam is reflected by a fixed ratio reflector. The two beams combine together to form a return beam which was then measured by the laser interferometer system. The control system of the stage is in closed loop state. The digital driving signal is sent step by step through computer to servo controller manually, and the outputs of laser interferometer and integral sensor of the stage are read at the same time. The reading of the latter comes from computer, so that it reflects a comprehensive property of the scanning stage including the effects from signal modulator of capacitance sensor, servo controller, DPT driver, A/D converter and interface board. Fig. 5.12 shows the plot of calibration curves of one cycle measurement in the $x$ axis. The results of $y$ axis calibration are as same as that of the $x$ axis. From the curves it can be seen that the output of laser interferometer has a little bit drift, but the integral sensor and servo system are very stable and have a good linearity and repeatability which will be further discussed later. At each end of the curves there is a blank section in which input driving signals are out of the working range for DPT driver, so that no output is measured. By comparing these two curves from the laser interferometer and the integral capacitance sensor, one can obtain a displacement of $0.85 \mathrm{~nm}$ corresponding to one bit of $\mathrm{A} / \mathrm{D}$ converter for data sampling and 3.4 $n m$ corresponding to one bit of coarse $\mathrm{D} / \mathrm{A}$ converter of servo module, resulting in a minimum driving step of $0.425 \mathrm{~nm}$ through fine $\mathrm{D} / \mathrm{A}$ converter.

The measurement loop in the $z$ axis was calibrated with a commercial Qucensgate Instruments Digital Piezoelectric Translator which was very well calibrated by manufacturer to nanometer level and of a nonlinearity of less than $0.2 \%$. To separate it with the DPT used for $z$ translator, we call it standard DPT (SDPT) here. A schematic diagram of the calibration set is shown in Fig. 5.13. The 


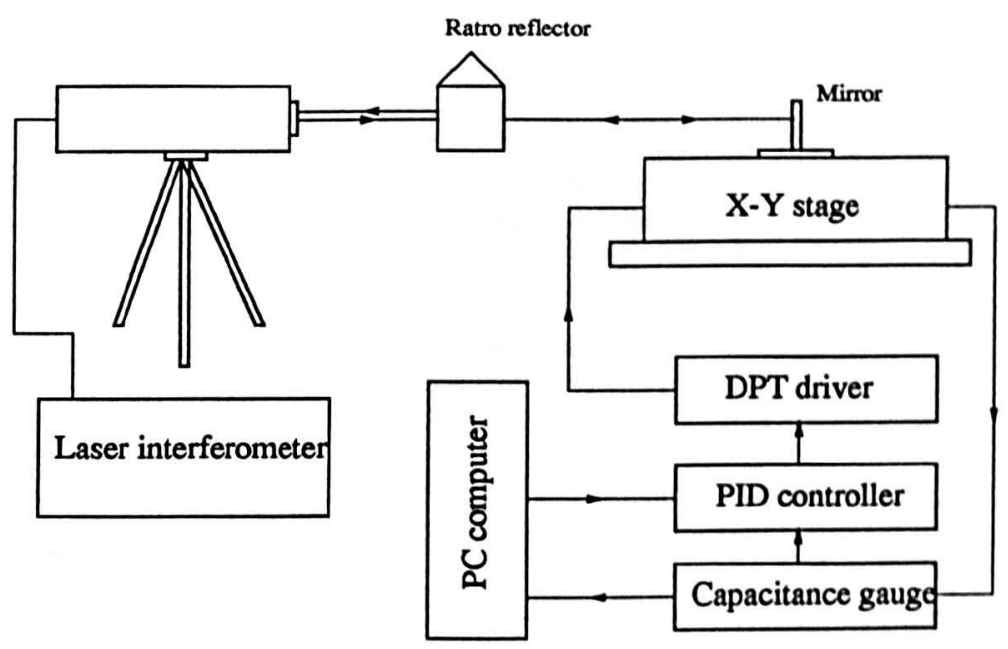

Figure 5.11: Schematic diagram of calibration system of the $x-y$ stage

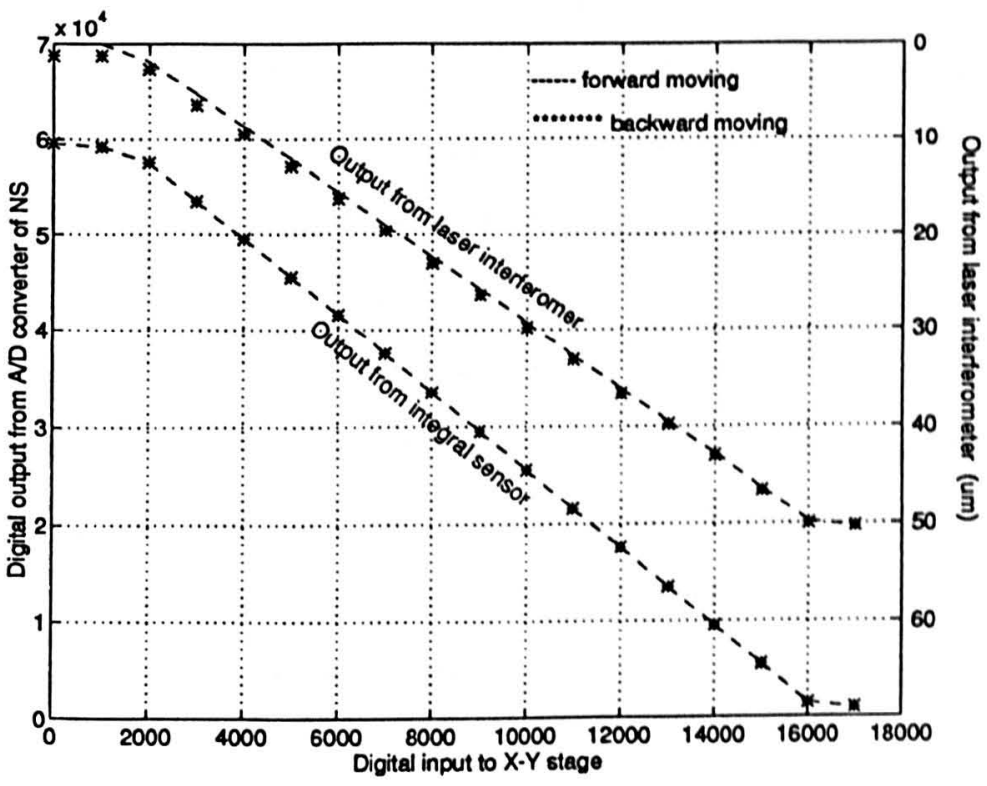

Figure 5.12: Plot of the calibration curves of the X-Y stage in $x$ axis 


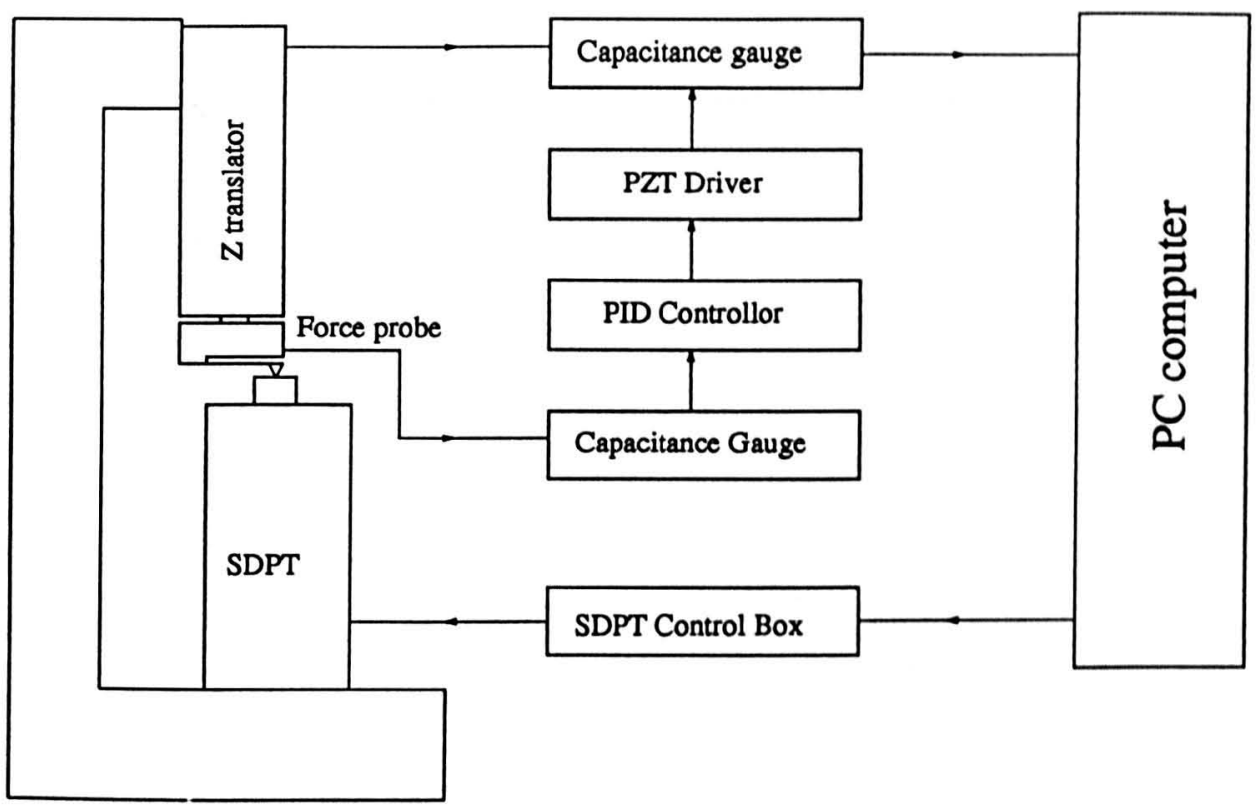

Figure 5.13: Schematic diagram of calibration set for measurement system in the $z$ axis

SDPT is located under the $z$ translator on which the force probe is mounted. The $z$ translator is thus positioned that the tip of force probe is in contact with the top surface of expansion element of SDPT and the system to be calibrated is in fully closed loop control. Then read the outputs from the sensor of $z$ translator through computer while sending a linear driving signal to SDPT. Fig. 5.14 shows the results of the calibration. It gives a resolution of $0.21 \mathrm{~nm}$ corresponding to one bit of A/D converter for the $z$ translator. To assess the results of the calibration, A standard 30nm step specimen manufactured by Rank-Taylor-Hobson was measured on both the SFM and Rank-Taylor-Hobson Nanostep and results were compared. Profiles of the step are presented in Fig. 5.15 showing a good agreement between these two instruments and specimen. 


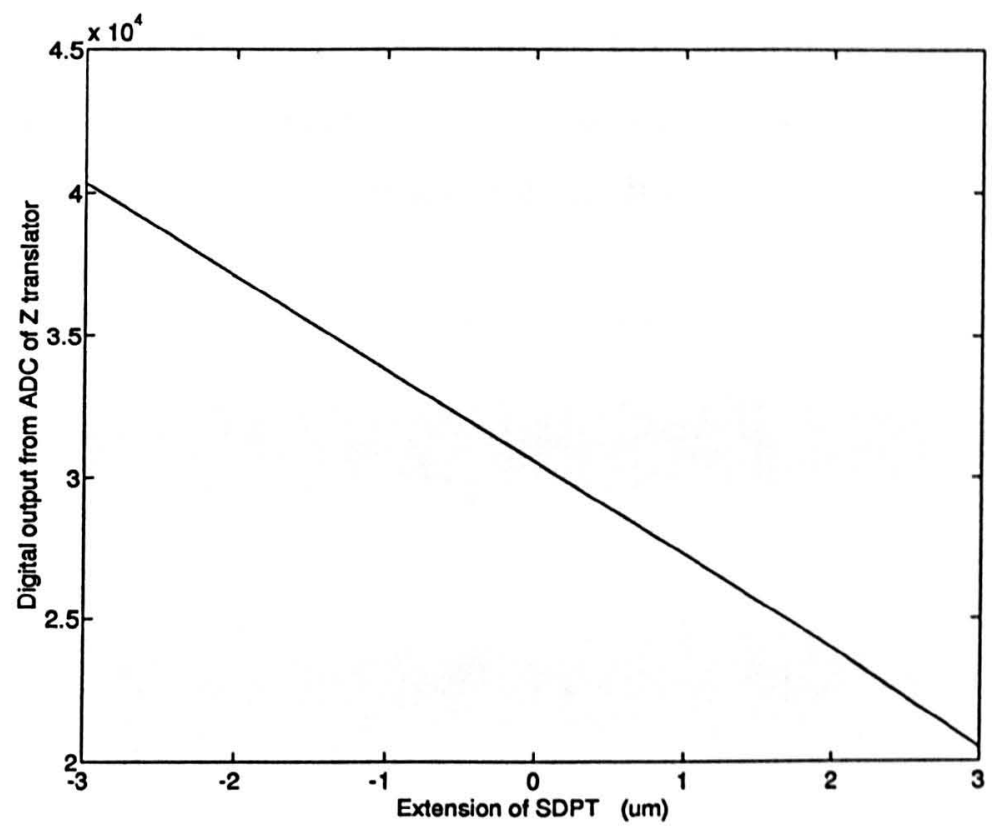

Figure 5.14: Plot of the calibration curves of measurement loop in the $z$ axis
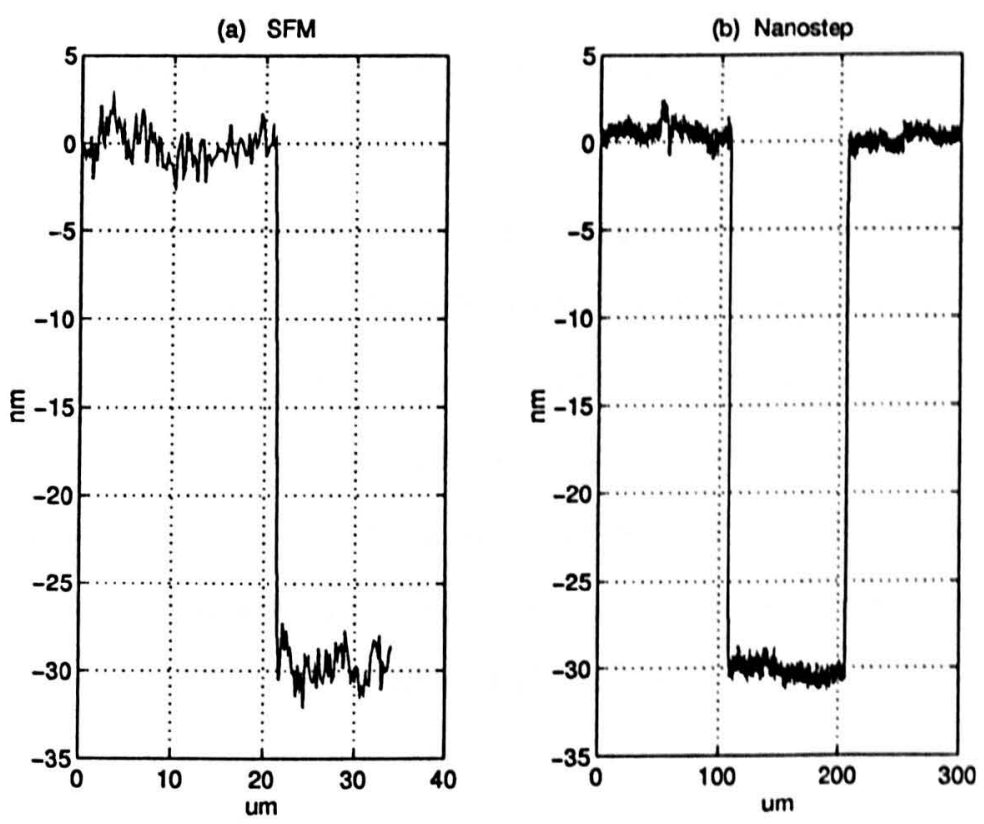

Figure 5.15: Plot of standard step profiles from SFM and Nanostep 


\subsubsection{Noise level}

To reveal the effects of various noise sources, the instrument outputs have been measured with the various components of the system being systematically activated. Fig. 5.16 (a) illustrates the noise signal from the $z$ axis Digital Piezoelec-

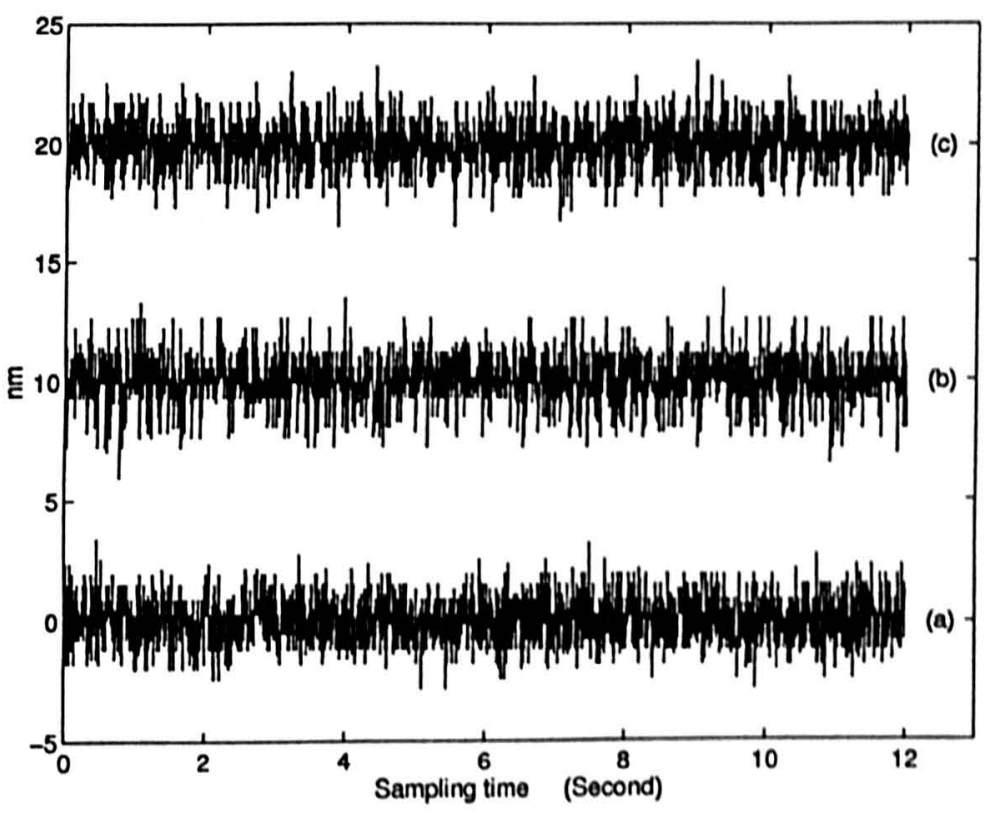

Figure 5.16: Noise level of the instrument system

tric Translator only. This includes the noise components of piezoelectric actuator, integral capacitance sensor and NanoSensor module. Connecting the output of the capacitance gauge of the force probe to the servo controller which, in turn, goes to DPT driver results in the noise of Fig. 5.16 (b). Finally, the total noise level for the system in the $z$ axis while the tip is in contact with a stationary silica specimen under full closed-loop control is plotted as 5.16 (c) which shows a peakto-peak value of less than $4 \mathrm{~nm}$ and a RMS value of $1.03 \mathrm{~nm}$. For Fig. 5.16 (a) and $5.16(\mathrm{~b})$, the RMS values are $1.00 \mathrm{~nm}$ and $1.14 \mathrm{~nm}$, respectively. Although measurement results might imply an increased noise level as more components involved in the noise measurement system in the $z$ axis, the increase is insignificant for the $z$ axis probe. Therefore, the noise magnitude of the output of DP'T 
system can be considered to be a main contribution to the noise level. The noise level of Queensgate Instruments NanoSensor module is related to bandwidth by

$$
N_{\text {tot }}=N_{f} \sqrt{B}
$$

here, $N_{\text {tot }}$ is total noise ( $n m$ RMS), $N_{f}$ is noise factor [13] (in our case, $N_{f}=$ $\left.0.02 \mathrm{~nm}_{z}^{-\frac{1}{2}}\right)$ and $B$ is bandwidth $\left(H_{z}\right)$. We used the default $5 \mathrm{kH}$ bandwidth set by manufacturer giving a noise level of $1.41 \mathrm{~nm}$ RMS from equation (5.1). This bandwidth is excessive for our application and was reduced in subsequent experiments.

It is obvious that if subnanometer resolution is to be achieved, the system noise level has to be cut down further. Usually it is very difficult to reduce the noise level by improving the design of electronics. To add a low-pass filter at the output port of NanoSensor module of the DPT is another possible option. However, these electronics modules we used for SPM are all commercial kits and it was inconvenient to modify them. To increase the resolution of our SFM, a simple box-car averaging filter has been adopted in the process of data acquisition. Because the $x-y$ stage is controlled digitally, the computer can do sampling and averaging repeatedly to the output of DPT at each increment. The experimental results are plotted in Fig. 5.17 which shows the output of $z$ translator while the force probe is in contact with a stationary silica specimen.

It is clear that as the averaging times increases, the noise level drops down accordingly. Fig. 5.18, gives the relationship of averaging times, noise level and sampling speed. At the point corresponding to 500 times averaging in Fig. 5.18, the noise level drops to approximately $0.176 \mathrm{~nm}$ (RMS). After that, improvements in noise level will result in unacceptably high scan times. Averaging 1000 times at each data point, one can obtain the noise level of $0.1 \mathrm{~nm}$ (RMS). As a result, angstrom resolution can be achieved, implying a precision of one part in $10^{5}$ with a full DPT vertical range of $15 \mu \mathrm{m}$. However, it has to be emphasised that this decreases speed of data acquisition as well. It can be seen from Fig. 5.18 that the 


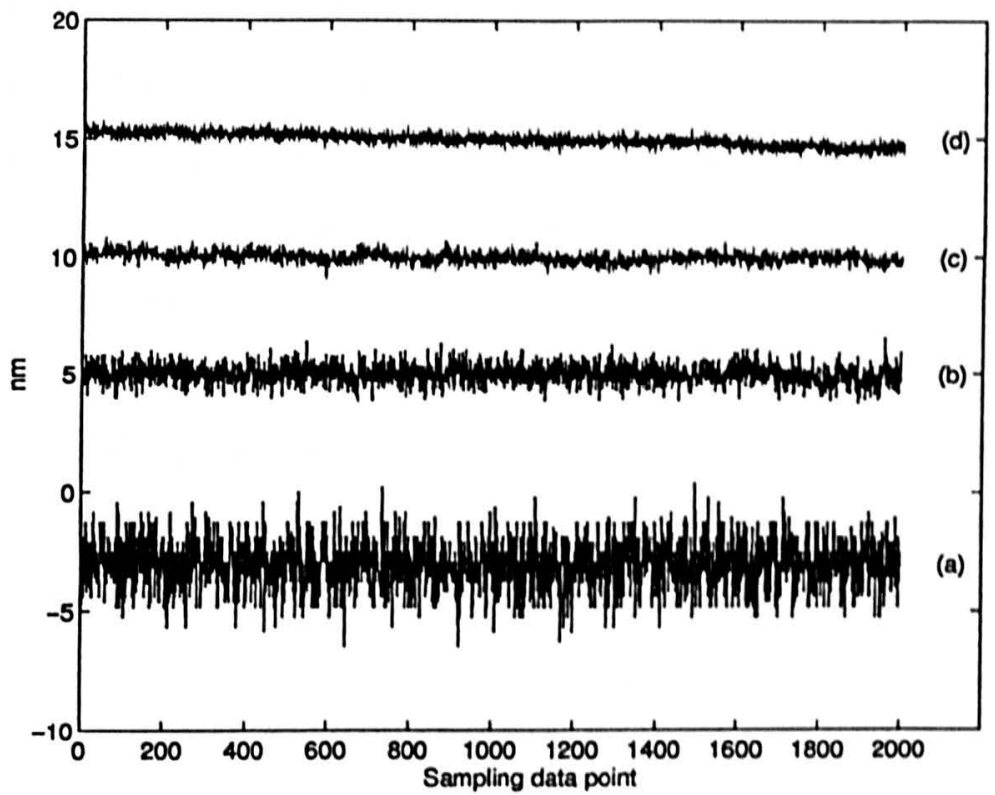

Figure 5.17: Noise level corresponding to sampling processes with various averaging times for each data point. The averaging times for these plots: (a) - zero, (b) $-10,(\mathrm{c})--50,(\mathrm{~d})-1000$.

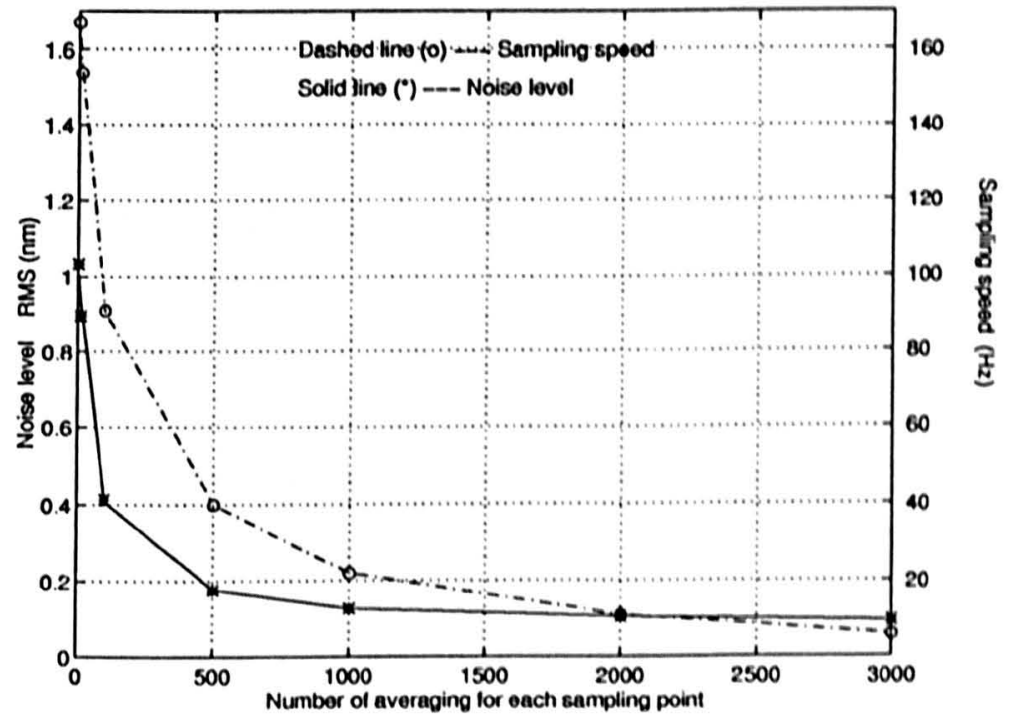

Figure 5.18: Noise level and speed of data acquisition vs. averaging times 
speed of data acquisition declines from $160 \mathrm{H}_{z}$ to $40 \mathrm{H}_{z}$ when averaging times goes up from zero to 500 . With this speed ( $40 H_{x}$ ) of data acquisition, about 17 minutes are needed for scanning a image of $200 \times 200$ data points. A small drift can be seen from the noise signal plots in Fig. 5.17. Least squares fit straight lines reveal a drift of less than $0.01 \mathrm{~nm} / \mathrm{s}$. For example, Fig. 5.17 (c), corresponding to 500 averaging times and $40 \mathrm{H}_{\mathrm{x}}$ data acquisition rate over a period of 50 seconds, shows a drift less than $0.5 \mathrm{~nm}$.

The noise magnitudes of the $x-y$ scanning stage were measured by monitoring the output from the two sensors when the stage stayed still. Because noise levels are similar in two axes, only the signals from the sensor of $x$ axis are presented here. Fig. 5.19 (a) is the noise signal measured while the $x-y$ stage in openloop state, i.e. the noise of integrated capacitance sensor and the NanoSensor modulator, showing a RMS value of $1.18 \mathrm{~nm}$. The bandwidth of NanoSensor modules used for the $x-y$ stage is set at $500 H_{z}$, therefore, the noise level is lower than that of the DPT. Fig. 5.19 (b) corresponds to the noise level of the stage under full closed-loop control, giving a peak-to-peak magnitude of $5 \mathrm{~nm}$ and a RMS value of $1.586 \mathrm{~nm}$. With a full scanning range of $50 \mu \mathrm{m}$, a precision of better than one part in 30,000 can be achieved over this relatively broad sensor bandwidth.

\subsubsection{Stability and repeatability}

Because a relatively long scanning time is required for measurement, a good stability is essential for precise measurement. The thermal effect is usually considered to have a big influence on instrument stability, and so, the thermal drift of the instrument was measured. A semiconductor thermal sensor was fixed onto the SFM to monitor the temperature fluctuation. The temperature of the laboratory is servo controled, so it fluctuated periodically. The SFM was in a closed loop control state where the tip is kept in contact with a specimen, so that the effects of the whole measurement loop is included. Then the outputs from the 


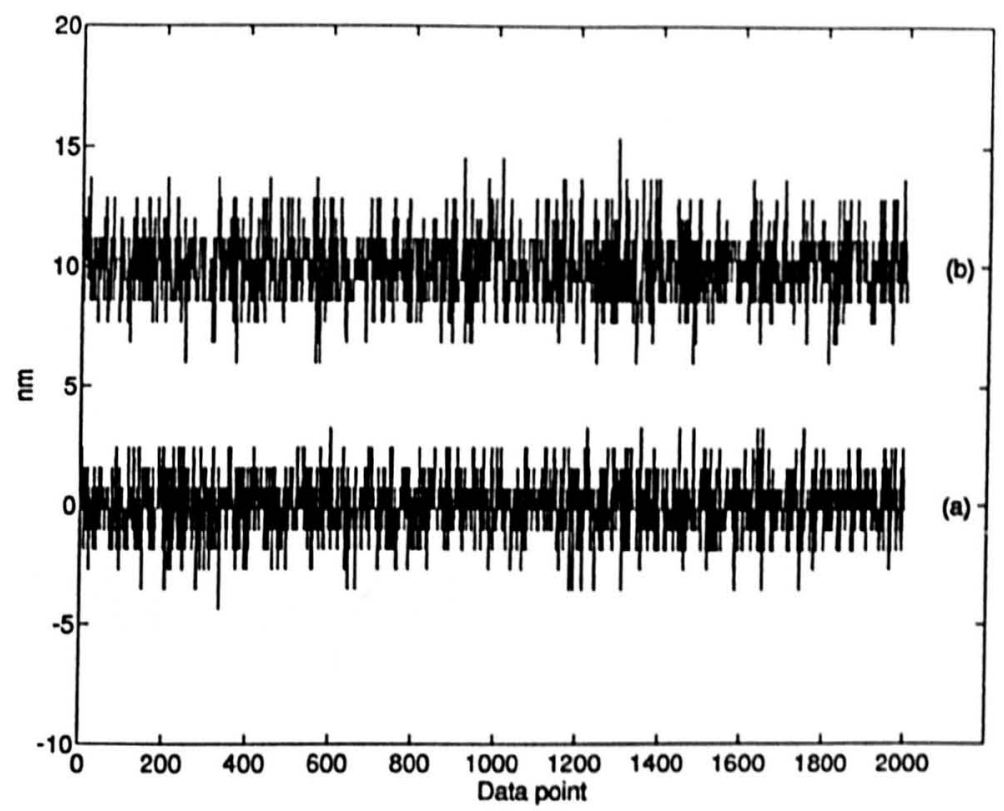

Figure 5.19: Noise levels of $x-y$ scanning stage in the $x$ axis. (a) - open-loop control state, (b) - closed-loop control state

thermal sensor and $z$ transducer of SFM were sampled through a computer simultaneously. Fig. 5.20 (a) shows a drifting curve of the system output, indicating a $50 \mathrm{~nm}$ drift in a period of $100 \mathrm{~min}$. To separate the effects of temperatures with others, the data from $z$ transducer was leveled, and is compared with the temperature changes, see Fig. 5.20 (b). From this, the thermal influence can be seen clearly that the output drifting follows the temperature fluctuation with a small lag in time. The temperature was measured to have a peak-to-peak fluctuation magnitude of $0.22^{\circ} \mathrm{C}$ with a fluctuation period of 11 minutes. The instrument has a periodic drift of $10 \mathrm{~nm}$ in magnitude, corresponding to a drift rate of $50 \mathrm{nmK}^{-1}$, with the same period as the temperature. The specification of the NanoSensor used in DPT gives a thermal drift of $40 \mathrm{~nm}^{-1}$, which corresponds to $8.8 \mathrm{~nm}$ drift in $0.22 C^{\circ}$ temperature variation. Besides the NanoSensor, the force probe is also a capacitance sensor which inevitably is also affected by temperature fluctuations although this is expected to be smaller. Therefore it is 

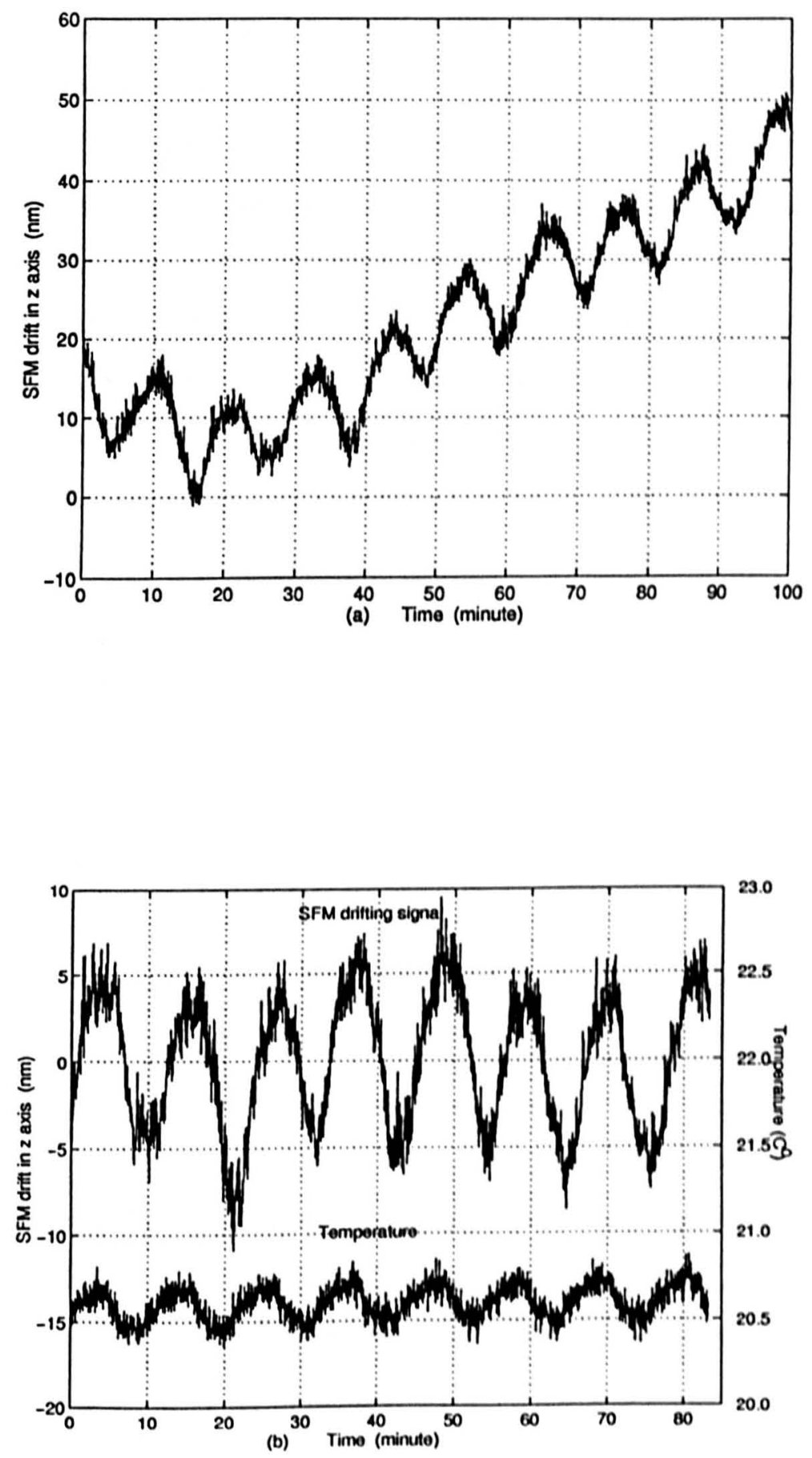

Figure 5.20: Plots of system thermal drift 
clear that the thermal sensitivity of the structure of measurement loop is much less than that of capacitance sensors.

To evaluate the linearity of the $x-y$ stage, we did a ramp scan over a range of $40 \mu \mathrm{m}$ by supplying a linear digital driving signal from computer to the $x-y$ scanning stage system in $x$ and $y$ axes simultaneously with the feedback loop closed and reading the outputs from the integral capacitance sensors. The scan trace is shown in Fig. 5.21. By fitting each straight line section in ramp scan plot with least square straight line, the maximum deviation point between ramp trace and fitted line is found to be $3.71 \mathrm{~nm}$ corresponding to a nonlinearity of $0.001 \%$ in a range of $40 \mu \mathrm{m}$. The outlines of deviation details are shown in Fig. 5.21 by leveling the ramp trace. The deviation data points looks much like noise evenly distributed along its centre line, and its peak-to-peak magnitude is within the noise level of the stage (see Fig. 5.19). From this, it appears that a nonlinear error of less than one nanometer in full scan range of $50 \mu \mathrm{m}$ is achievable in each axis.
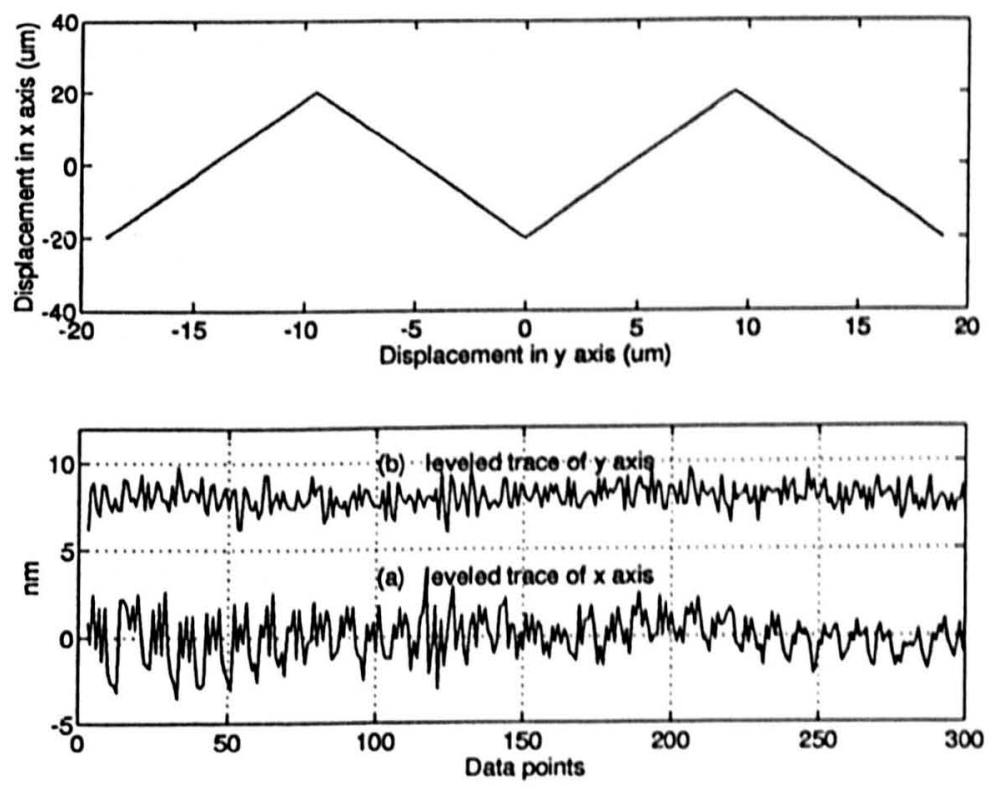

Figure 5.21: Ramp scan trace for analysis of linearity 
To demonstrate the repeatability of the $x-y$ stage, two repeat meander pattern scans over a small area of $44 \times 44 \mathrm{~nm}$ are shown in Fig. 5.22, which are the same scan pattern as we used for surface measurement with the scanning force microscope. The data are sampled and plotted without any artificial processing and relocating. Again no filtering is applied in data sampling and processing. Based on these data, the position errors of these parallel lines are calculated by fitting the grating traces with least square straight lines. It gives a maximum space error of $0.31 \mathrm{~nm}$ between adjacent lines and a maximum inclined error of $0.77 \mu \mathrm{rad}$ for single line. The errors of the second scan are worked out to be quite similar with the first one. By comparing these two scans, the maximum position difference between the corresponding lines (line 1 in scan 1 to line 1 in scan 2) comes out to be $0.246 \mathrm{~nm}$. In Fig. 5.22, the individual bits of the output of capacitance gauge are clearly visible, and it is obvious that the peak-to-peak magnitude of the trace fluctuation is within 3 bits of the output of $A / D$ converter with each bit corresponding to a displacement of $0.875 \mathrm{~nm}$. These indicate that the $x-y$ stage is capable of positioning and scanning with an accuracy and repeatability at subnanometer levels. To assess the repeatability of the instrument in vertical axis, two repeat profiles of an optical grating surface are plotted in Fig. 5.23, which are obtained from a continuous forward and backward scan. The data were sampled from the $z$ translator while the stage was scanning. Again no filtering was applied. From these data, the mean point to point vertical difference between the two profiles comes to be $0.3125 \mathrm{~nm}$, resulting in a relative error of 0.4 $\%$ over a vertical range of $66.56 \mathrm{~nm}$. These include not only the errors from the $z$ translator and force probe system but also the vertical errors of $x-y$ stage. From the evaluation above, it is clear that the instrument system has an repeatability at nanometer levels in all three axes. 


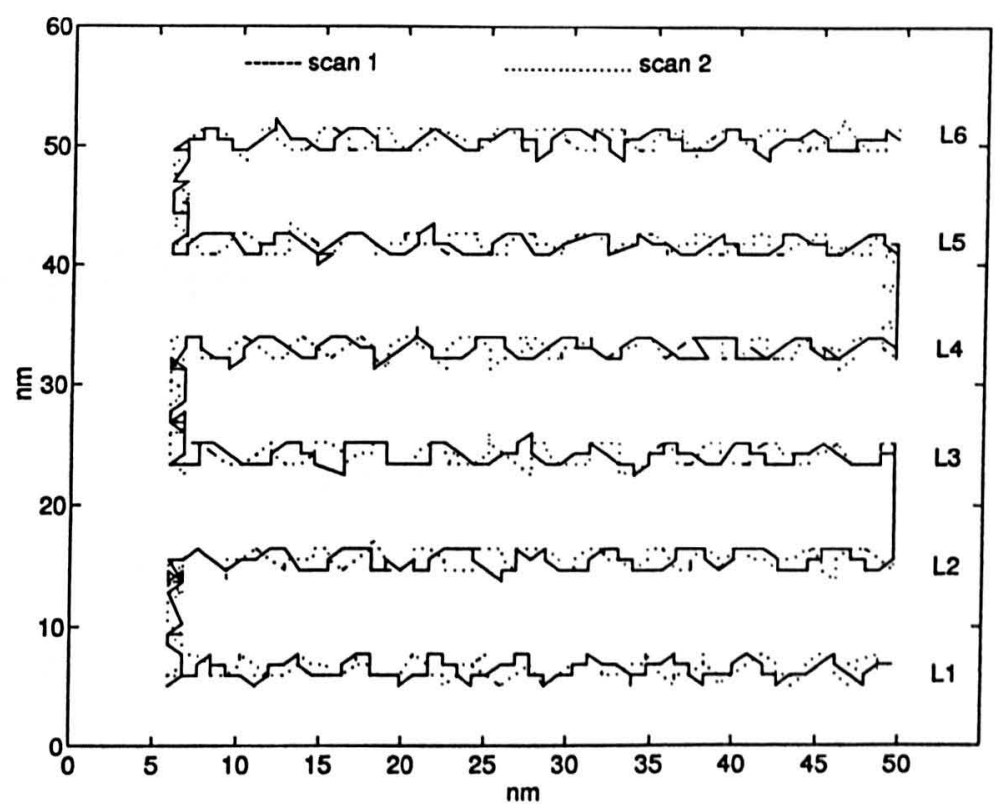

Figure 5.22: Plots of two meander pattern scans

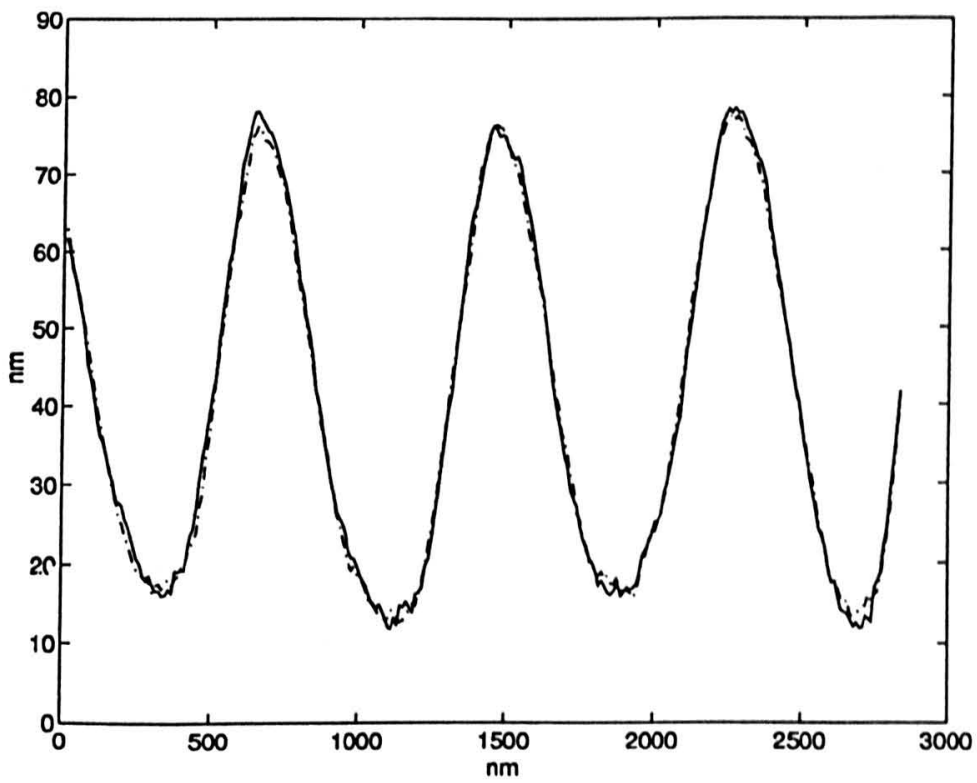

Figure 5.23: Plot of two optical grating surface profiles obtained from a continuous forward and backward scan 


\section{Bibliography}

[1] Y. Kuk and P.J. Silverman, 1989, Scanning tunneling microscope instrumentation, Rev. Sci. Instrum. 60(2), $165-180$

[2] J.E. Griffith, D.A. Grigg, M.J. Vasile, P.E. Russell, and E.A. Fitzgerald, 1992, Scanning probe metrology, J. Vac. Sci. Technol. B10(4), 674 - 679

[3] D.R. Baselt, S.M. Clark, M.G. Youngquist, C.F. Spence and J.D. Baldeschwieler, 1993, Digital signal processor control of scanned probe microscopes, Re». Sci. Instrum., 64 (7), 1874 - 1882

[4] R.C. Barret and C.F. Quate, 1991, Optical scan-correction system applied to atomic force microscopy, Rev. Sci. Instrum. 62(6), 1999 - 1999

[5] C.V. Newcomb I. Flinn, 1982, Improving The Linearity of Piezoelectric Ceramic Actuators, Electron. Lett. 18442 - 443

[6] H.Kaizuka, 1989, Application of capacitor insertion method to scanning tunneling microscopes, Rev. Sci. Instrum. 60, 3119 - 3122

[7] J.E. Griffith, G.L. Miller and C.A. Green, 1990, A Scanning Tunneling microscope with a capacitance-based position monitor, J. Vac. Sci. Technol. B8 (6), 2023 - 2027

[8] H. Yamada, T. Fujii and K. Nakayama, 1989, Linewidth Measurement by a New Scanning Tunneling Microscope, Jpn. J. Appl. Phys. 28, 2402 - 2404 
[9] E. Clayton Teague, Russell D. Young, Frederic Scire, and David Gilsinn, 1988, Para-flex stage for microtopographic mapping, Rev. Sci. Instrum. $59(1), 67-79$

[10] E. Clayton Teague, 1989, The National Institute of Standards and Technology molecular measuring machine project: Metrology and precision engineering design, J. Vac. Sci. Technol. B7(6), 1896 - 1902

[11] T.H. McWaid and J. Schneir, 1994, The Development of a Calibrated Atomic Force Microscope, Proceedings of ASPE 1994 Spring Topical Meeting.

[12] S.T. Smith, D.G. Chetwynd and D.K. Bowen, 1991, Calibration of heightmeasuring probes, SPIE Vol. 1573., 225 - 236

[13] System NanoPositioning User's Guide, by Queensgate Instruments Ltd.

[14] S.T. Smith, S. Harb, V. Eastwick-Field, Z.Q. Yao, P.N. Bartlett, D.G. Chetwynd and J.W. Gardner, 1993, Tribological properties of electroactive polymetric thin film bearings, Wear, $169,43-57$

[15] T.H. Waid, J. Fu, T.V. Verburger and J.F. Song, 1993, A comparison of stylus, interferometric and scanning probe measurements, Proceedings of ASPE 1993 Annual Meeting, 259 - 261

[16] J.M. Bennett, V. Elings, and K. Kjoller, 1993, Recent developments in profiling optical surfaces, Applied Optics, 32 (19), 3442 - 3447

[17] K. Huster, 1994, Surface measuring techniques for nanotechnology, M.Sc. Thesis, University of Warwick

[18] K. Lindsey, S.T. Smith, and C.J. Robbie, 1988, Subnanometre surface texture and profile measurement with NANOSURF 2, CIRP Ann., 37, 519 522 
[19] J.D. Garratt and S.C. Bottomley, 1990, Technology transfer in the development of a nanotopographic instrument, Nanotechnology, 1, $38-43$ 


\section{Chapter 6}

\section{Surface measurement}

\subsection{Surface topography}

Specimens with different surface geometrical characteristics, feature scales and material properties were measured for instrument testing. All the images shown below are sampled with $200 \times 200$ data points which we feel to be sufficient for imaging purposes. These specimens are chosen to illustrate metrological capability over a broad engineering specimen range. Tips of radius 1.0 (normal stylus tip) and $<0.1$ (Berkovich tip) were used. Fig. 6.1 and 6.2 show the SEM images of these two tips.

The contact forces between the tip and specimen were controlled at about $200-400 n N$ in the measurements. The contact forces are determined by both the stiffness of the cantilever and its deflection. The stiffness can be directly calculated out according to the dimensions of the cantilever beam or calibrated experimentally by adding small mass on the cantilever while monitoring the deflection of the cantilever. The deflection in measurement is determined by the difference betwisen the initial output of force probe and setting point of servo modul. 


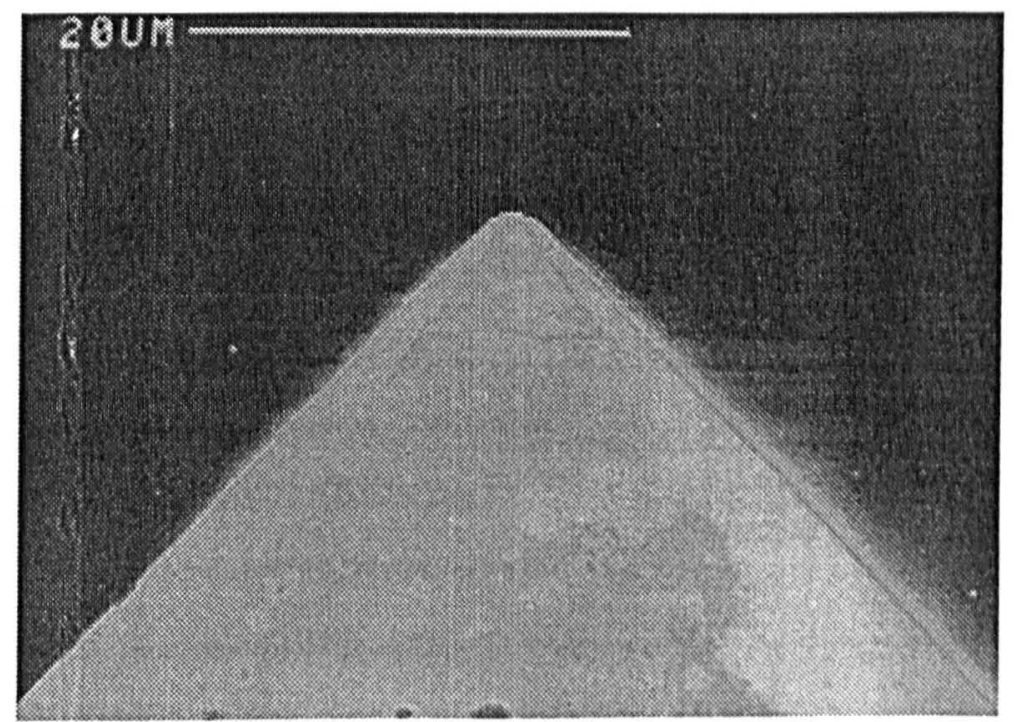

Figure 6.1: SEM image of normal stylus tip of radius $1 \mu \mathrm{m}$

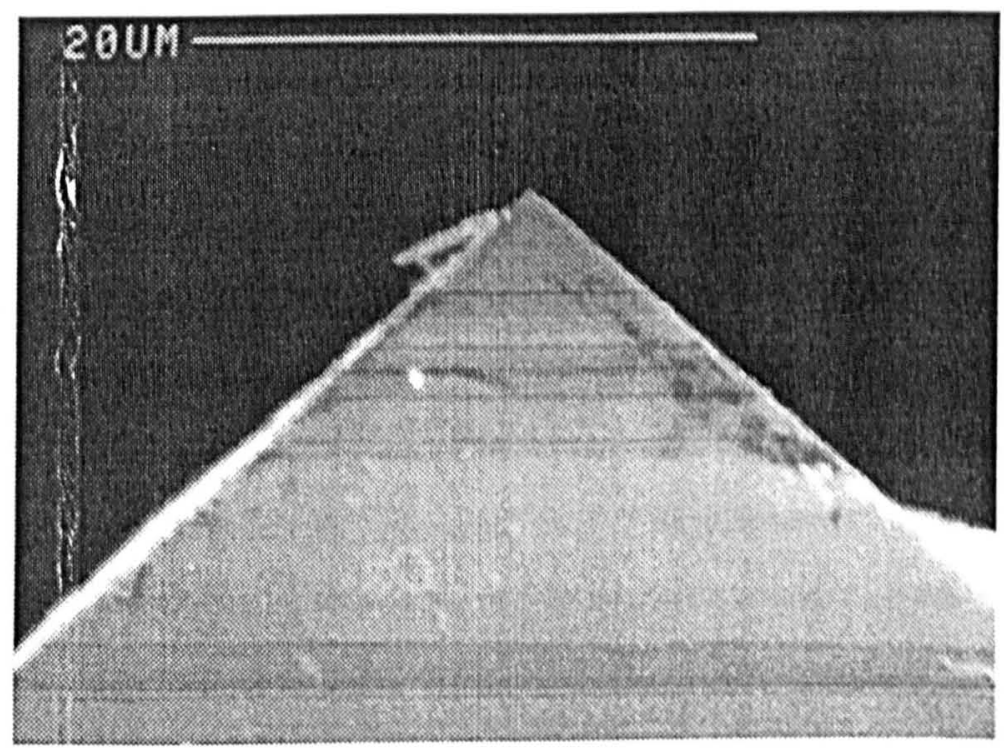

Figure 6.2: SEM image of Berkovich tip of radius $<0.1 \mu \mathrm{m}$ 


\section{Optical grating surface}

Two standard optical grating specimens were scanned using the metrological SFM. One of them is a specimen of 1216 lines/mm produced by Rank-TaylorHobson with sinusoidal undulations on the surface. Fig. 6.3 (a) shows a 3D view of a scanning area of $6.6 \mu \mathrm{m} \times 6.6 \mu \mathrm{m}$. Its section profile, Fig. 6.3 (b), illustrates the depth of grating lines of about $65-70 \mathrm{~nm}$ and the distance between the lines of about $820 \mathrm{~nm}$. Fig. 6.4 is a 2D map of this grating surface and Fig. 6.5 is a SEM image from the same specimen. Comparing these two images, there is no distortion can be seen in the 2D map. Fig. 6.6 is the image of a cross grating surface of 2160 lines $/ \mathrm{mm}$ which is a standard specimen for SEM calibration. All the images above are measured using the Berkovich tip shown in Fig. 6.2. The stylus tip was also tried, but no surface features could be obtained for the specimen shown in Fig. 6.6.

\section{Polymeric films}

Because of the low contact force, the SFM is capable of measuring soft specimen surfaces. As an example, electroactive polymeric films presently under investigation for use as micro-actuator bearings [1] were measured. Poly(N-methylpyrrole) polymer is eletrochemically deposited from an electrolyte solution containing the monomer onto a gold film that is, in turn, evaporated on to the convex face of polished glass substrates. The friction properties of the polymer film bearings are believed to be dependent upon the load, film thickness and bearing geometry according to present theoretical and experimental analysis. A group of the bearings were chosen for the SFM measurements. These bearings, labeled as D2, D9 and D12 in the reference [1], were grown using same polymer, electrolyte, growth potential and oxidation state, but different growth charges. The thicknesses of the polymeric films were claimed to be $0.49 \mu m$ (D9), $0.43 \mu m$ (D12) and 0.12 $\mu m$ (D2). Fig. 6.7, 6.8 and 6.9 demonstrate the surface topographic images of these polymer films. Polymer agglomerates are clearly shown and a big difference 
in surface features can be observed. The initial results of the friction experiment [1] indicate that the coefficient of friction tends to rise with the thickness of the polymeric films. From the images, the thicker the film, the rougher the surface appears. It can be suspected that the surface features may also have an influence on the frictional or tribological properties of the bearings. Therefore, the metrological SFM may be very useful for tribology research.

\section{Others}

Some other specimens with various surface topographic features have also been measured with the metrological SFM to demonstrate its potential application areas. Fig. 6.10 presents an image of a well polished Zerodur optical flat. Tiny scratches with depths of 3 or $4 \mathrm{~nm}$ can be observed in the surface image, which are believed to have been produced in the polishing process. The $R_{q}$ and $R_{a}$ values of surface roughness was calculated to be $0.69 \mathrm{~nm}$ and $0.54 \mathrm{~nm}$ from these data. Fig. 6.11 shows an isometric plot of integrated electronics on a silicon chip with scan range of $30 \times 30 \mu \mathrm{m}$ and peak-to-valley variations of about $2 \mu \mathrm{m}$. With this measurement technique, fabrication quality can be monitored precisely. Another example of application is the surface quality control of semiconductor thin film growing process. Fig. 6.12 shows a surface topographic map of $S_{i} / S_{i 0.8} G_{e 0.2} / S_{i}$ heterostructure sample. The tiny pits on the surface are believed to be caused by strains produced in the process of multilayer growing. 

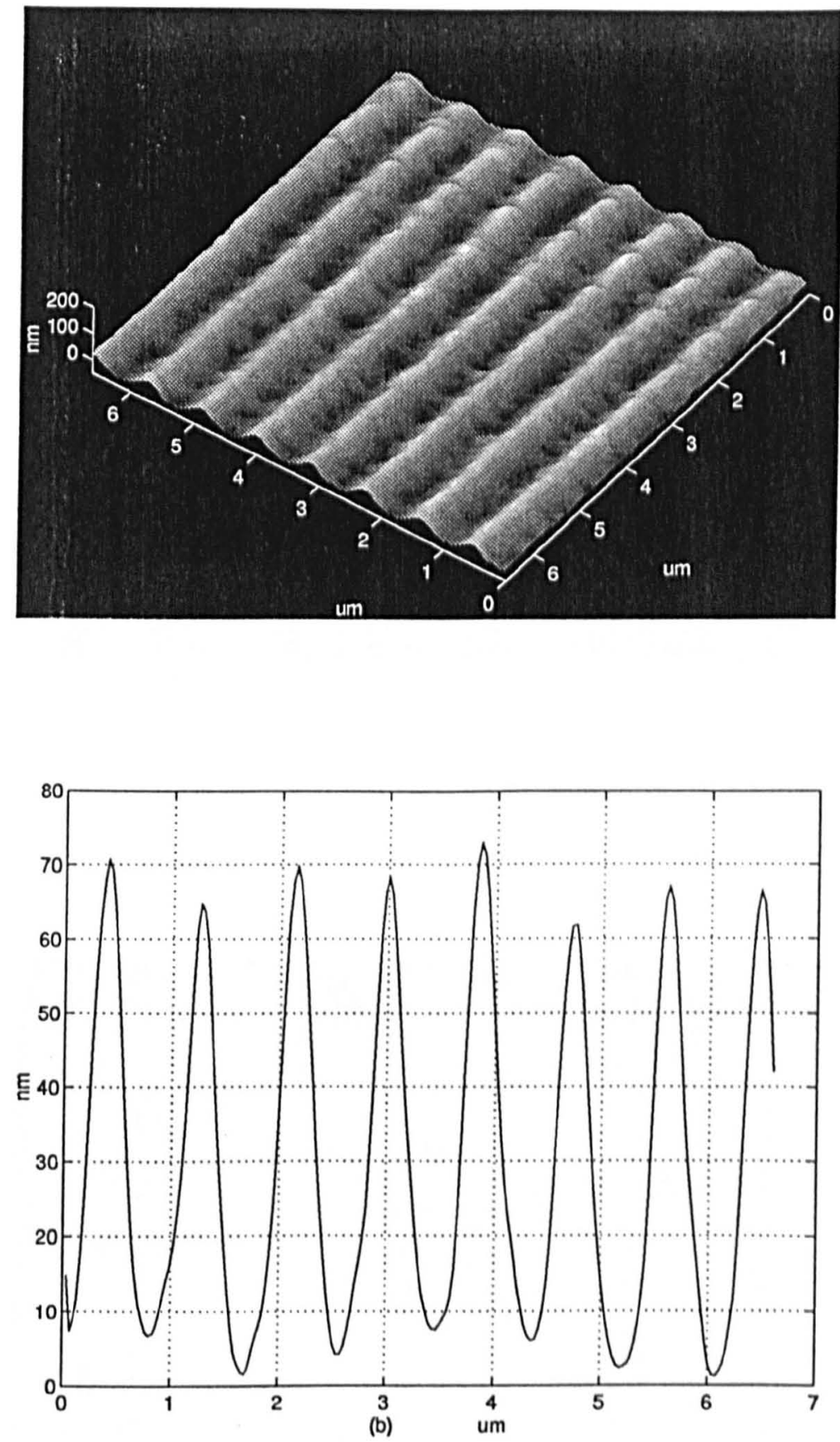

Figure 6.3: Image of optical grating surface. (a) 3D view, (b) Section profile 


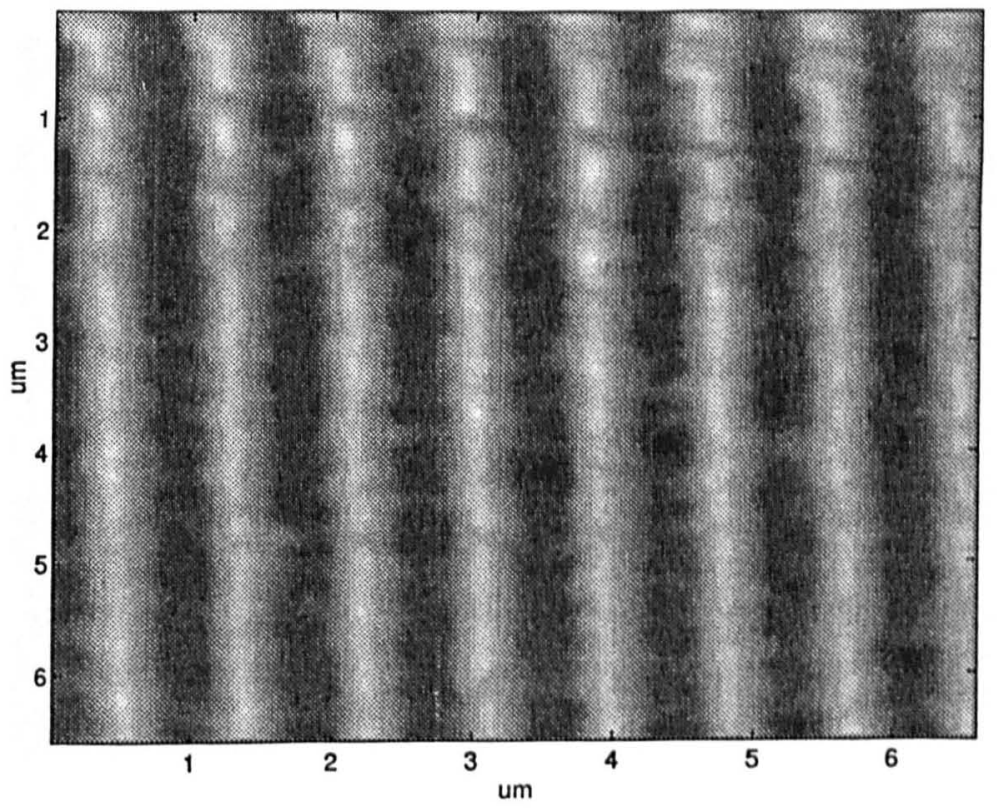

Figure 6.4: $2 \mathrm{D}$ view of the same grating surface as in Fig.6.3

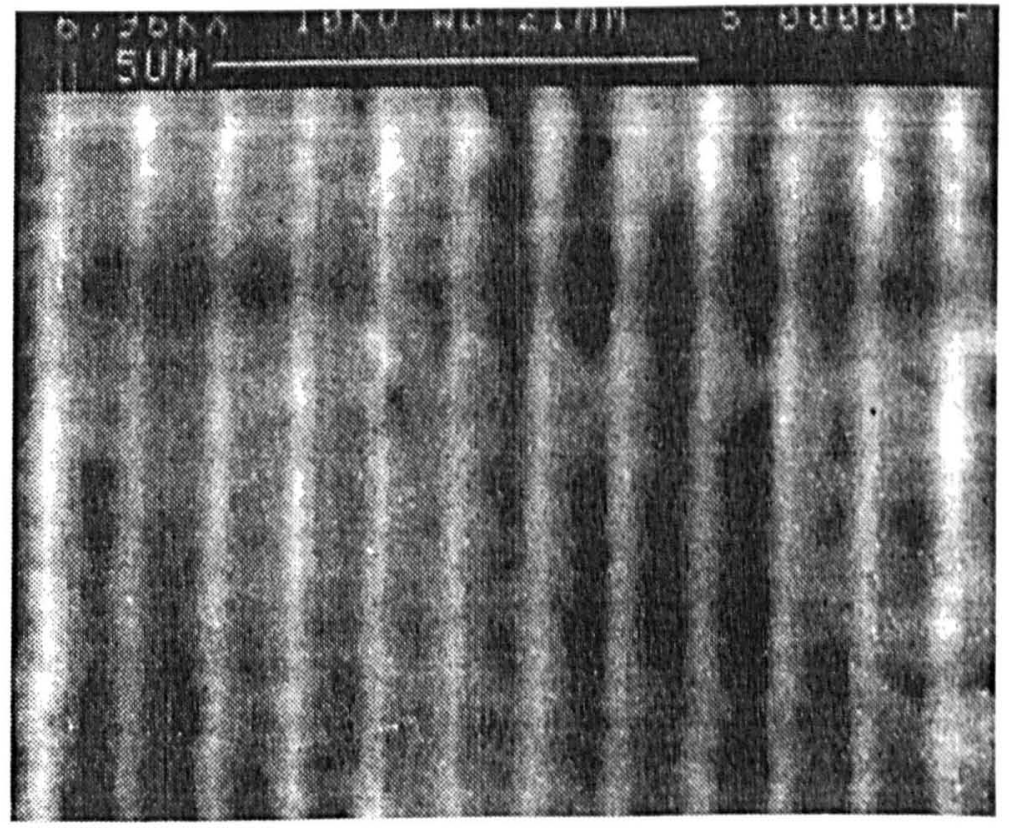

Figure 6.5: SEM image of the same grating surface as in Fig.6.3 


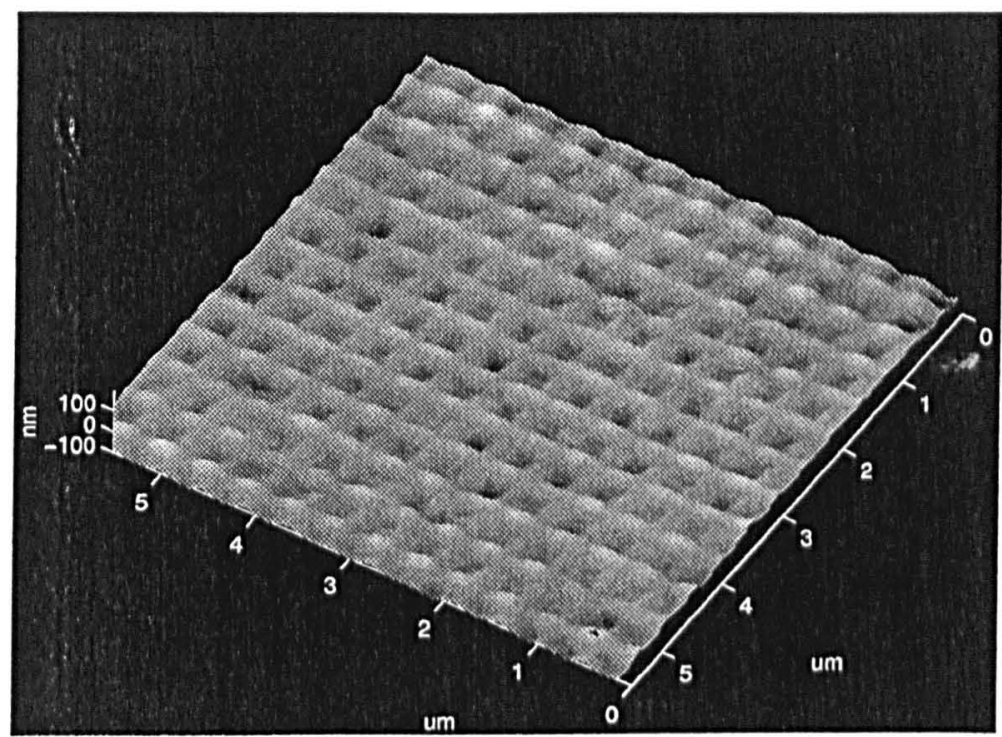

Figure 6.6: SPM image of standard gratings of 2160 lines $/ \mathrm{mm}$ for SEM calibration

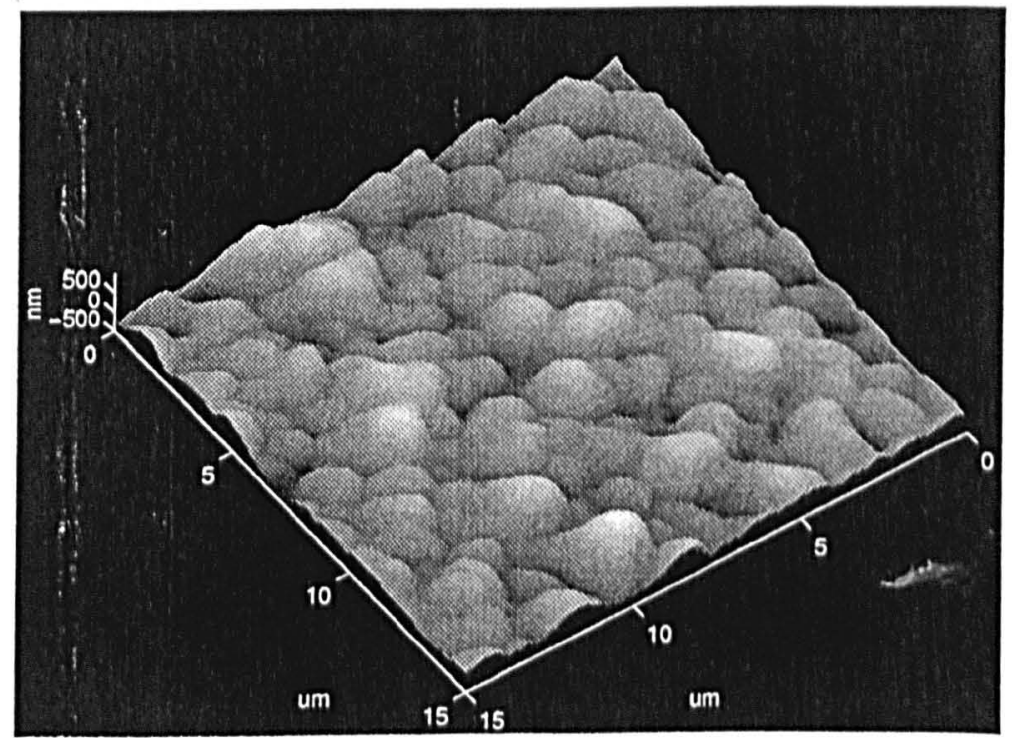

Figure 6.7: SPM image of polymer film (D9) 


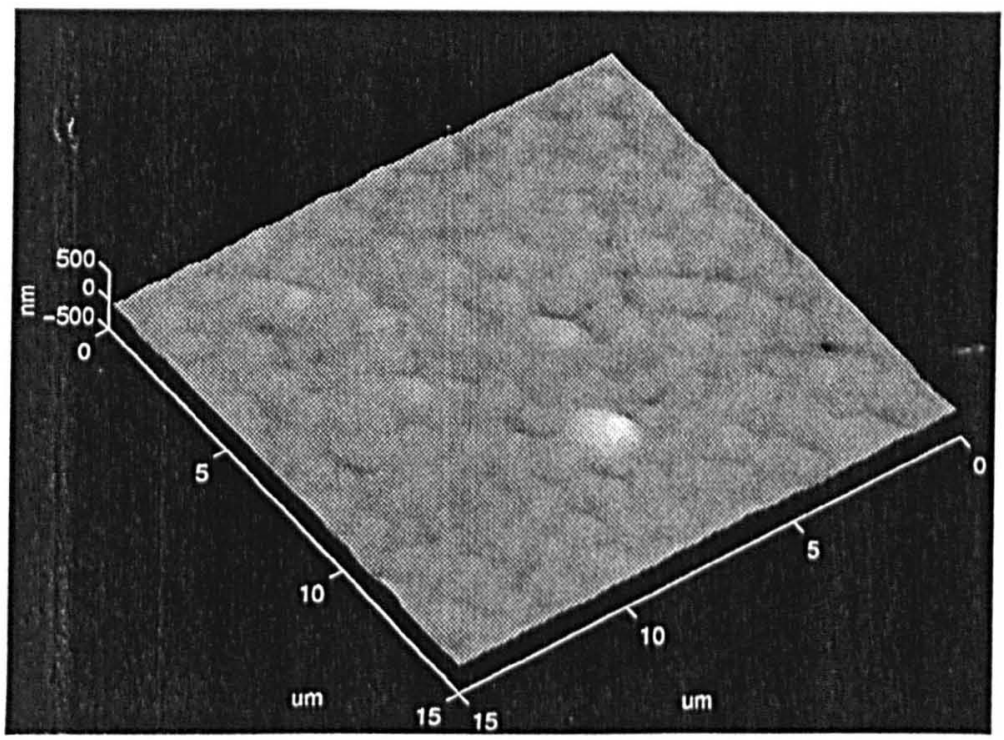

Figure 6.8: SPM image of polymer film (D12)

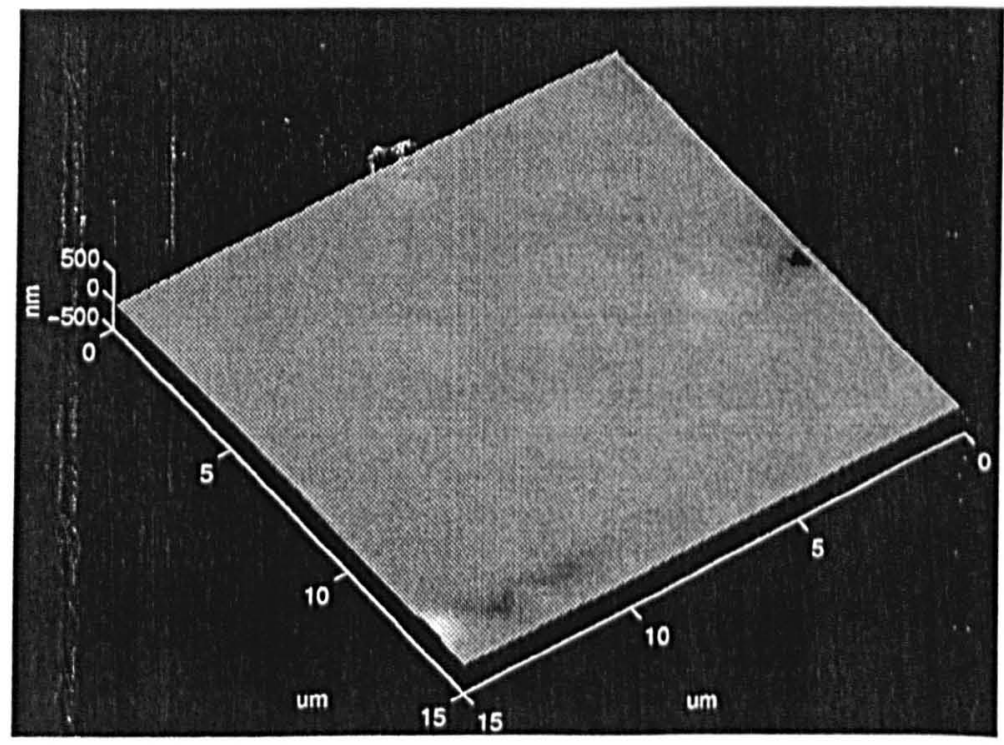

Figure 6.9: SPM image of polymer film (D2) 


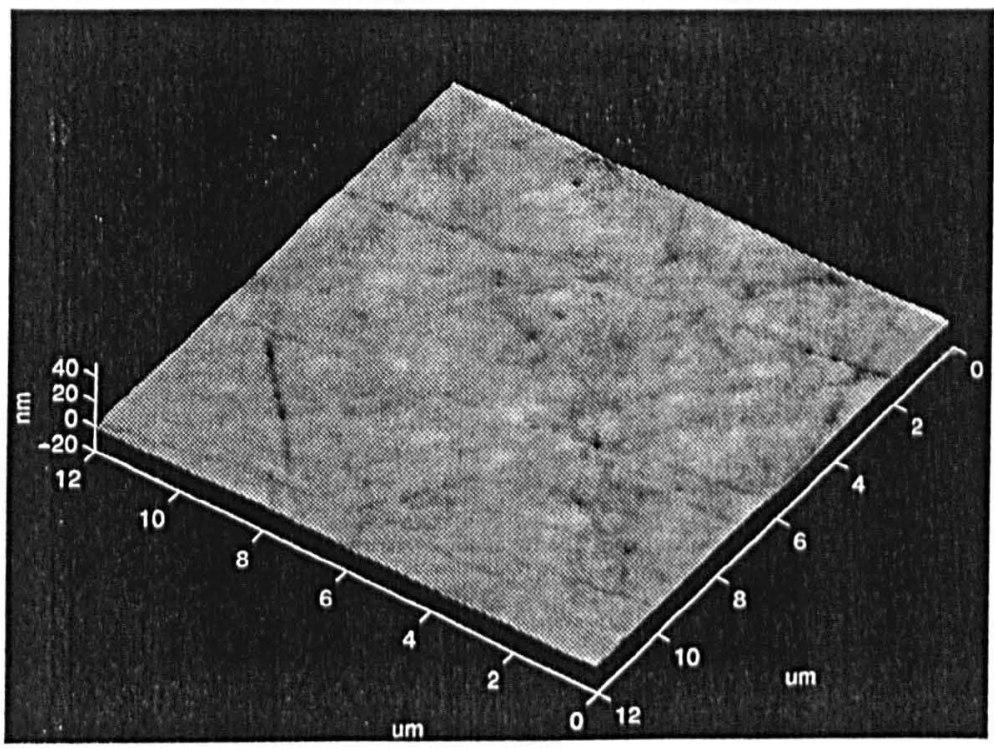

Figure 6.10: SPM image of a well polished Zerodur optical flat

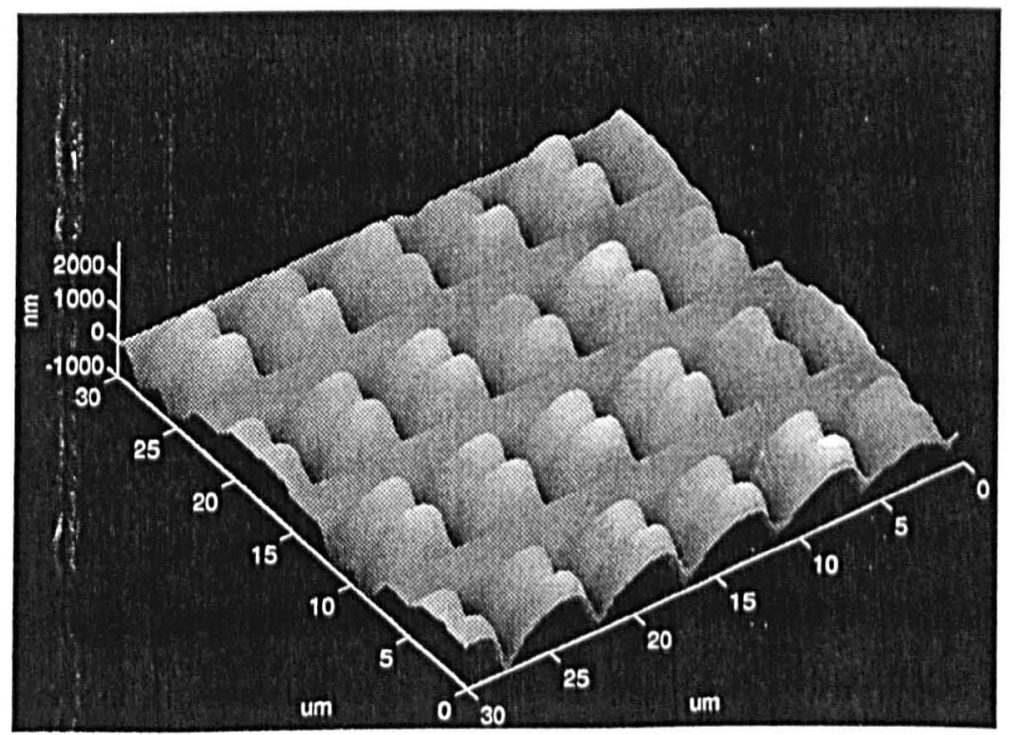

Figure 6.11: 3D image of electronic components on a silicon chip 


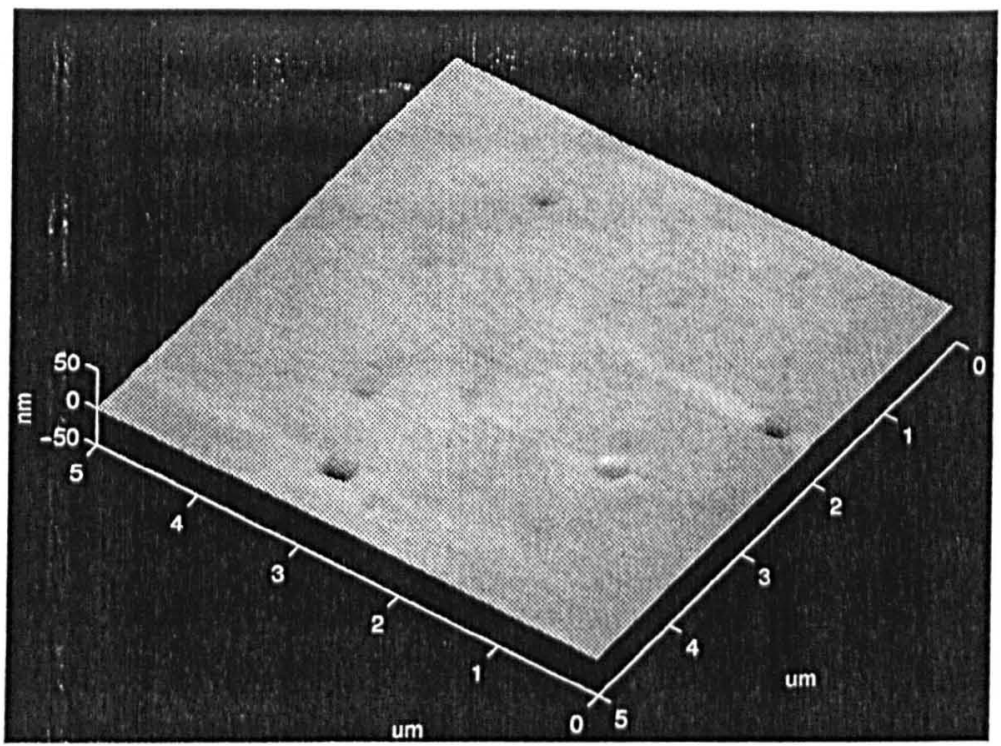

Figure 6.12: Surface topography of $S_{i} / S_{i 0.8} G_{e 0.2} / S_{i}$ heterostructure sample

\subsection{Surface roughness measurement}

\subsubsection{Introduction}

As mentioned in Chapter 1, surface roughness is a significant feature of merit for characterization of samples. It can provide information about sample fabrication processes and input to theories for prediction of mechanical, optical and electronic performance. As surface features have become smaller, it is more difficult to obtain meaningful surface parameters, because the heights of surface feature are comparable with instrumental noise levels and the lateral dimensions of surface textures are close or smaller than the lateral resolution of many conventional instruments. Therefore, many new techniques have been developed to meet the increasing demands on the measurements of ultra-smooth surfaces. However, these techniques are based on different working principles, measuring surface features from different point of view. The reliabilities of these instruments become more and more concerned by both designers and users. An easy way to assess the reliabilities is to compare the measurement results, obtained using different tech- 
niques. Some research work investigating the relationship between the techniques have been reported in recent years [2] [3] [4].

In our experiments, three advanced surface measurement instruments are used, Nanostep, X-ray reflectometer and metrological SFM, and their measurement results are compared.

\subsubsection{Instrumentation}

\section{Nanostep}

The Rank Taylor Hobson (RTH) Nanostep 2010 is a stylus instrument designed to meet the requirements of the microelectronics industry for micro profiling. It is modeled on a design developed by Lindsey et al. at the National Physical Laboratory [5]; the configuration of the commercial instrument has been described by Garratt and Bottomley [6]. In this instrument the stylus is stationary and the sample moves. The sample is held on a stage which has movements for lateral positioning and levels the sample in the profile direction by translating a wedge located under the stage. The sample stage rests on a Zerodur slideway of inverted $V$ shape. A motor drives a Zerodur slave carriage, also resting on the slideway, through a micrometer screw and a coupling, pushing the sample stage to which it is connected. Five Teflon pads on the underside of the slave carriage and sample stage provide low-friction-bearing surfaces. This results in a super-precision slideway system with noise levels on the order of $50 \mathrm{pm}$. Much of the measurement loop is constructed of Zerodur, giving the instrument a low temperature coefficient of approximately $7 \mathrm{~nm} /{ }^{\circ} \mathrm{C}$. In our experiments, a diamond pyramidal stylus truncated to a $0.1 \times 2.5 \mu \mathrm{m}$ tip is used, which is attached to a LVDT pickup. Maximum length of traverse is $50 \mathrm{~mm}$, with speed limited by the bandwidth of the modified Talystep transducer head $\left(8 k H_{x}\right)$. The system is interfaced via an electronic unit to Talydata 2010. 
X-ray method for ultra-smooth surface measurement is a relatively new technique. At very small incident angles, X-rays are totally reflected from solid surface. Above some critical angle the grazing incidence X-ray reflectivity (GIXR) falls rapidly. For a perfectly smooth and flat surface with no deposited layers and zero absorption the fall begins abruptly at the critical angle $\theta_{c}$ and is approximately proportional to $\theta^{-4}$ for angles well above $\theta_{c}$, where $\theta$ is incident angle. Departures from the ideal structure of up to few hundred nanometres may be measured. Surface roughness causes the reflectivity to fall off more rapidly [7]. Fig. 6.13 demonstrates the calculated specular curves for a Zerodur specimen with a perfect smooth surface and with added surface roughness, respectively. The reflective curves are simulated using the Bede Scientific Reflectivity Simulation program REFS. From this it can be seen that $1 \mathrm{~nm}$ (RMS) roughness difference on the surface can be easily identified. The Bede GXR1 Reflectome-

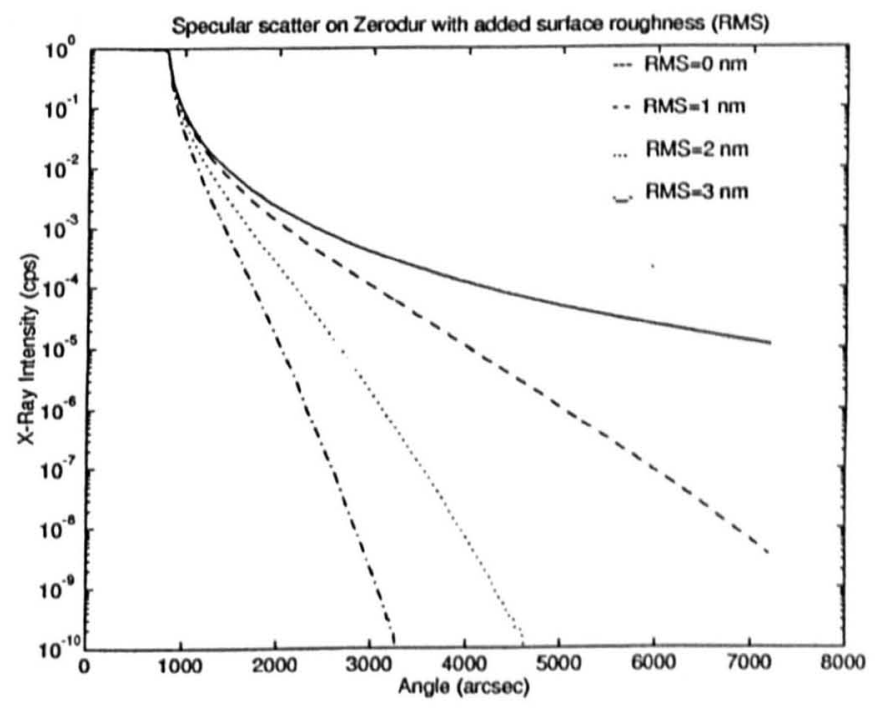

Figure 6.13: Calculated GIXR of a perfect smooth surface and the surfaces with added roughness

ter was used for the experiment. The instrument uses a conventional laboratory generator, a $C u K \alpha$ tube, running at $1.4 \mathrm{~kW}$. A beam conditioner is used to 
collimate the X-rays providing a incident beam with very small divergence, and a detector capturing the reflected beam. The sample stage is driven by motors through three micrometers arranged in a $Y$ shape to provide tilting in two directions. Care must be taken to align the specimen in angle to about 10 arcsec parallelism with the incident beam slit, and in height to about $10 \%$ of the beam height, requiring a precision of a few micrometres. No special specimen preparation is required. The instruments provides the ability to scan the specimen and the detector independently so that both specular and diffuse components of reflectivity can be measured.

\section{Scanning force microscope}

The metrological scanning force microscope has been described in detail in chapter 5 . The stylus tip of radius $1 \mu \mathrm{m}$ was used.

\subsubsection{Experiment process and results}

As mentioned above, the main objective of the experiment is to compare three complementary techniques for ultra-smooth surface measurement.

Zerodur was chosen as a desired material from the point of views of both ease of preparation and its potential application in precision engineering. It is a transparent, homogeneous glass-ceramic with both amorphous and crystalline phases. These two phases have opposite thermal expansion properties. By carefully annealing, an exceedingly low thermal expansion property can be achieved: about $1 \times 10^{-7} /{ }^{\circ} \mathrm{C}$ for temperature between -30 and $70^{\circ} \mathrm{C}$. Besides, it is also dimensionally stable compared with other glasses or ceramics and has a reasonably high $E$ modulus ( $E=90 \times 10^{9} \mathrm{~N} / \mathrm{m}^{2}$ ) [8] [9]. Because of these properties, it has become increasingly popular in precision engineering for construction of ultra-high precision machine tools and metrology devices. For the machining property, Zerodur is much like conventional optical glasses, but it is much softer and of a relative low grinding specific energy, which means a relative low grinding force and a high 
machining precision. Moreover, Zerodur can be optically polished because the material is pore free and has similar hardness between its two phases.

The specimens were cut from a Zerodur bar of $40 \mathrm{~mm}$ in diameter which is sliced into wafers of $6 \mathrm{~mm}$ thickness using a diamond wheel slicing machine. Then they were chamfered and lapped on a Lapmaster lapping machine to get surface flat to about $1 \mu \mathrm{m}$ in whole surface area checked with the RTH Talysurf. The lapping paste used for flattening was a mixture of Alumina oxide $\left(\mathrm{Al}_{2} \mathrm{O}_{3}\right)$ powder of particle size $1.2 \mu \mathrm{m}$ and distilled water. Finally, the components were polished on a Lapmaster optical polishing machine. The polishing paste consists of Cerium oxide $\left(\mathrm{Ce}_{2} \mathrm{O}_{3}\right)$ powder with $1.2 \mu \mathrm{m}$ grit size and filtered distilled water of particle size $<0.3 \mu \mathrm{m}$. The polishing table which is supported on air bearings run at the speed of $50 \mathrm{rpm}$ for approximately 30 hours for each specimen until the desired flatness and smoothness were achieved. At this stage, the flatness was compared against a reference optical flat by assessing interference fringes when two surfaces were superposed under a helium monochromatic light source with a wavelength of $0.5 \mu \mathrm{m}$ with each fringe corresponding to one half of the wavelength. A flatness of one quarter of a band $(0.0073 \mu \mathrm{m})$ was achieved. Then, the specimens were cleaned using filtered distilled water and warm acetone.

Nanostep profiling and SFM topographic imaging are both contact measurements. The possible surface damages caused by stylus scratching have to considered in the design of the experiments. To avoid the potential influences of previous measurements, the experiments have to be arranged obviously in the sequence of non-contact measurement (GIXR), low contact force measurement (SFM) and relative high load contact measurement (Nanostep).

Five Zerodur specimens were prepared and measured with GIXR, SFM and Nanostep in the experiment. The results are listed in Table 6.1.

Fig. 6.14 shows the output signal of a specular scan from the GXR1 reflectometer. The surface roughness is assessed by comparing the measured specular scattering curve with simulated curve of the scattering from a model structure 


\begin{tabular}{|c|c|c|c|c|c|c|}
\hline Specimen & \multicolumn{3}{|c|}{ Nanostep } & \multicolumn{2}{|c|}{ SFM } & GIXR \\
\hline Samp/Surf & $\begin{array}{c}p^{2} \text { prile length } \\
(\mu \mathrm{m})\end{array}$ & $\begin{array}{c}R_{q} \\
(n \mathrm{~nm})\end{array}$ & $\begin{array}{c}R_{a} \\
(\mathrm{~nm})\end{array}$ & $\begin{array}{c}R_{q} \\
(\mathrm{~nm})\end{array}$ & $\begin{array}{c}R_{a} \\
(n m)\end{array}$ & $\begin{array}{c}R_{q} \\
(n m)\end{array}$ \\
\hline Zerodur 1/1 & 12.5 & 0.6 & 0.5 & 1.2 & 1.0 & 1.08 \\
& 100 & 1.0 & 0.7 & & & \\
& 400 & 1.1 & 0.8 & & & \\
\hline Zerodur 2/1 & 12.5 & 0.7 & 0.6 & 1.1 & 0.9 & 1.25 \\
& 100 & 1.1 & 0.8 & & & \\
& 400 & 1.4 & 1.0 & & & \\
\hline Zerodur 3/1 & 12.5 & 0.6 & 0.5 & 1.1 & 0.9 & 1.25 \\
& 100 & 0.7 & 0.6 & & & \\
& 400 & 0.7 & 0.5 & & & \\
\hline Zerodur 4/1 & 12.5 & 0.7 & 0.6 & 0.6 & 0.5 & 0.94 \\
& 100 & 1.0 & 0.8 & & & \\
& 400 & 1.3 & 1.0 & & & \\
\hline Zerodur 4/2 & 12.5 & 1.0 & 0.7 & 1.2 & 0.9 & 1.33 \\
& 100 & 1.2 & 0.9 & & & \\
& 400 & 1.4 & 1.0 & & & \\
\hline Zerodur 5/1 & 12.5 & 0.7 & 0.6 & 1.2 & 0.9 & 1.14 \\
& 100 & 0.9 & 0.7 & & & \\
& 400 & 1.1 & 0.8 & & & \\
\hline
\end{tabular}

Table 6.1: Results of the measurements by Nanostep, SFM and GIXR 
and adjusting the parameters until a good fit is obtained. The data are processed using the software package REFS [10]. The diffuse reflectivity is sensitive to the distribution and correlation length of the surface structure. This may be used to measure surface roughness to the highest precision and shortest cut-off wavelength known [7]. However, the data analysis is more complex. At the time of the thesis writing, only specular scattering analysis had been carried out.

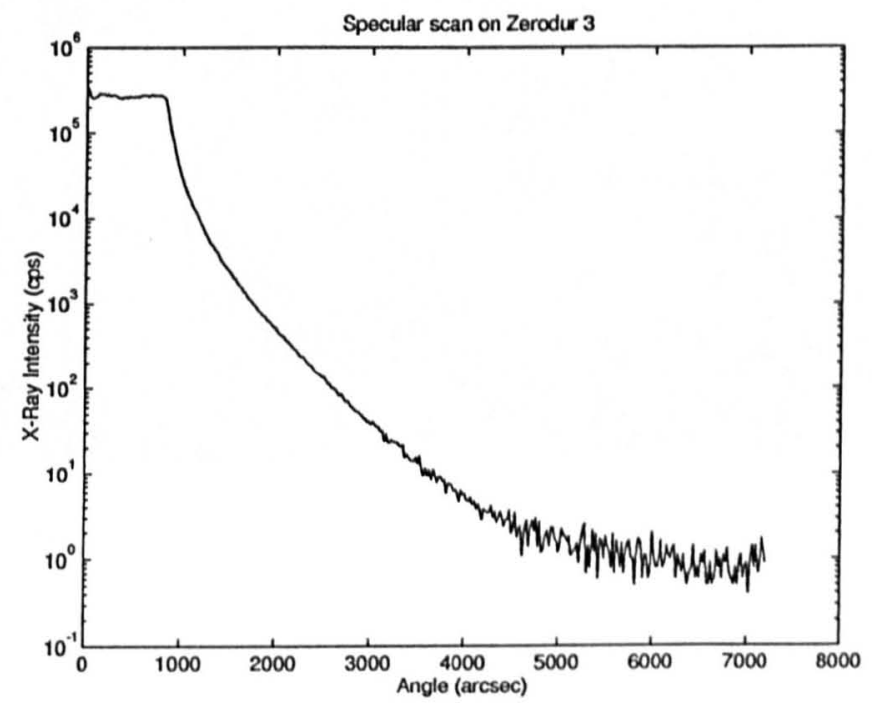

Figure 6.14: Curve of a specular scan from the GXR1 reflectometer

The GIXR method is powerful for statistical assessment of surface roughness in certain area, but measures surface roughness indirectly. The SFM provides a direct measurement on surface topography and gives point-to-point information of surface texture in a small area. More information can be extracted from the measurement. Fig. 6.15 shows a 3D image of the Zerodur surface (Zerodur 4/1), from which details of the surface feature can be seen clearly. Fig. 6.10 is the same measurement but viewed in smaller scale, from which the surface feature can be studied in macroscale. In this, the polishing scratches on the surface are clearly revealed. Therefore, the SFM method is very versatile for surface feature identification. The specimens were measured with scanning area 
$12.5 \mu \mathrm{m} \times 12.5 \mu \mathrm{m}$ and sampling data $200 \times 200$. The surface roughness was calculated two dimensionally and the results are given in the Table 6.1.

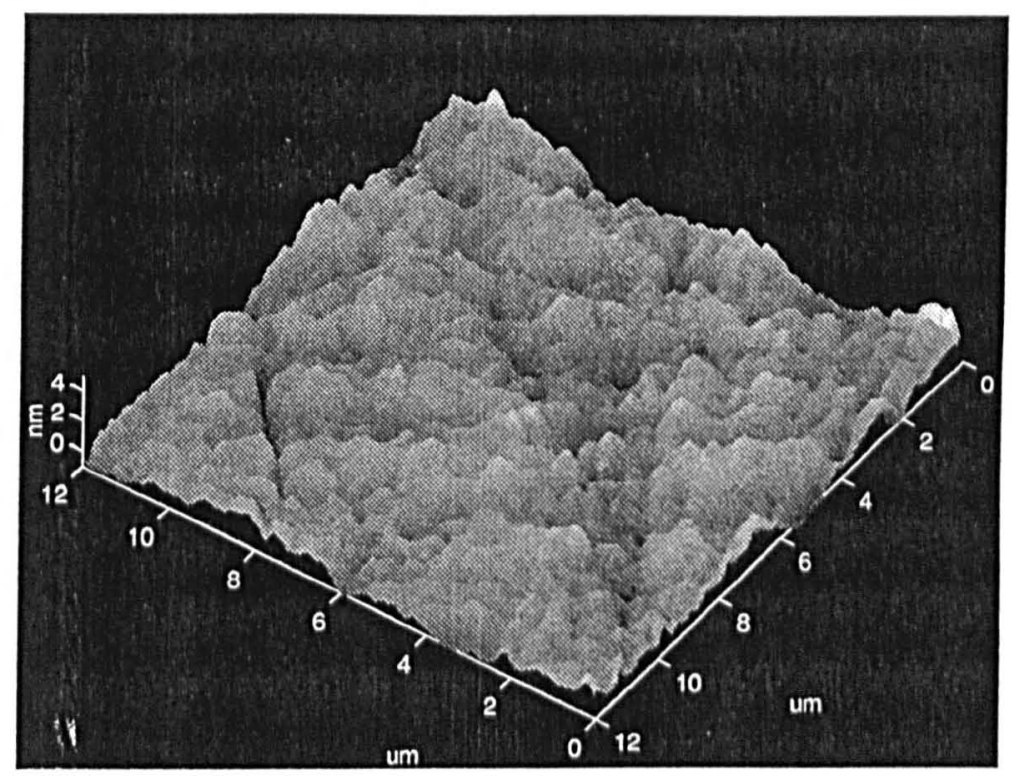

Figure 6.15: SFM image of Surface topography of Zerodur 4/1

Stylus instrument measures surface in two dimension profiling. It is considered to be a reliable measurement technique and two of the mostly used parameters $R_{a}$ and $R_{q}$ for assessment of surface roughness were defined based on the stylus technique. These parameters are now included in a number of national and international standards such as ISO4827, BS1134, ANSI B46.1 and DIN4768. Nanostep is an advanced version of the instrument, providing measurement with subnanometre resolution. On each specimen several measurements with different profiling length were conducted and the results are given in Table 6.1.

\subsubsection{Discussion}

From the results in Table 6.1, it can be concluded that the surface roughness measured with these three techniques, GIXR, SFM and Nanostep, are comparable. The measurement results are fairly close, which indicating that all these methods 
can be used as effective tools for surface roughness measurements in nanometre levels, although some deviations do exist. The possible reasons for the deviations can be analyzed in the following espects. From the results of the Nanostep it can be seen that the surface roughness will rise as profiling length increases. It is clear that as the measuring profile becomes longer, the effects of the surface features of relative long wave length will be included in roughness calculation, resulting in larger roughness values. Comparing the results of the Nanostep with SFM, the later indicates rougher surfaces in general. Data processing is considered to have some contribution to the difference. One dimensional $R_{q}$ and $R_{a}$ (Nanostep) are defined relative to a mean reference (least square) straight line along the profile. Two dimensional $R_{q}$ and $R_{a}$ (SFM) are referrd to a mean reference plane along the surface. In this case the reference line for each individual scanning trace may no longer meet the least square rule, so that some deviations may be generated. Both SFM and GIXR measure surface roughness over a area, but the roughness values of GIXR are all larger than that of SFM. This may be caused by effects of measurement ranges. Although the spot of the X-rays is only a few micrometres, it may extend to about $1 \mathrm{~cm}$ on the sample surface due to the very small incident angle. As same as the effect of profiling length, the larger area the measurement covers, the larger the surface roughness value will be.

\subsection{Discussion and conclusion}

A newly developed prototype metrological scanning force microscope has been introduced in previous and this chapters with the description of design strategy and a preliminary performance evaluation. Subnanometer resolution, stability and repeatability in all three axes are demonstrated. Because of the application of the high precision displacement position sensors in three axes, this SFM has a capability of not only imaging but also doing metrological measurement on precision engineering surfaces. The capability of directly providing areal information 
of surface characteristics is one of the advantage of SFM over stylus instruments. The low contact force makes it possible for use on soft materials without causing significant damage to the specimen. Furthermore, if other physical probe sensors are used in this SFM, various material property information may also be provided with similar accuracy.

This first prototype of the instrument is made of inexpensive material of aluminium, randering it susceptible to thermal variations. Thermal effect is inevitable for the measurement at nanometer or even subnanometer levels conducted in a normal temperature controlled laboratory. The next prototype of the instrument will be built with the materials of ultralow thermal expansion properties, such as Zerodur glass ceramic, fused silica, or Invar alloy. 


\section{Bibliography}

[1] S.T. Smith, S. Harb, V. Eastwick-Field, Z.Q. Yao, P.N. Bartlett, D.G. Chetwynd and J.W. Gardner, 1993, Tribological properties of electroactive polymetric thin film bearings, Wear, 169, $43-57$

[2] T.H. McWaid, J. Fu, T.V. Vorburger and J.F. Song, 1993, A comparison of stylus, interferometric and scanning probe measurements, Proceedings of ASPE 1993 Annual Meeting, 259 - 261

[3] J.M. Bennett, V. Elings, and K. Kjoller, 1993, Recent developments in profiling optical surfaces, Applied Optics, 32 (19), 3442 - 3447

[4] K. Huster, 1994, Surface measuring techniques for nanotechnology, Diploma Thesis, Chemnits University

[5] K. Lindsey, S.T. Smith, and C.J. Robbie, 1988, Subnanometre surface texture and profile measurement with NANOSURF 2, CIRP Ann., 37, 519 522

[6] J.D. Garratt and S.C. Bottonley, 1990, Technology transfer in the development of a nanotopographic instrument, Nanotechnology, 1, $38-49$

[7] D.K. Bowen and B.K. Tanner, 1993, Characterization of engineering surfaces by grazing incidence x-ray reflectivity, Nanotechnology, 4, 175 - 182

[8] R. Brehm, J.C. Driessen, P. v.Grootel and T.G. Gijsbers, 1985, Low thermal expansion material for high precision measurement equipment, Precision 
Engineering, 7, $157-160$

[9] S.T. Smith, 1988, Mechanical systems in nanometre metrology, Ph.D thesis, University of Warwick, U.K.

[10] REFS - Reflectivity Simulation Software, Bede Scientific Instruments Ltd. 


\section{Chapter 7}

\section{General discussion and}

\section{conclusion}

Nowadays, scanning probe microscopy is no longer a new concept in the area of precision engineering. Since the first STM was developed in 1982, a large group of SPMs have b een explored, which employ various sensing techniques to measure surfaces with ultrahigh resolutions in both vertical and lateral directions based on some of physical or chemical interaction. Although many successful results have been reported in which atomic resolution has been achieved, there are still a lot of improvements to be done and problems to be solved before SPM can be used for high precision surface measurement. The metrological scanning force microscope introduced in this thesis is an example of the efforts for the improvement of SPM technique in the aspect of instrumentation. Based on the development of this SFM system, some general questions are discussed in following.

\subsection{Metrological issues}

Because of the inherent nonlinearity of piezo actuators which are used in all the SPMs to provide scanning and contour following motions, the poor metrological capability of the SPMs developed thus far limits their application for quantita- 
tive measurement. Without a great improvement in its metrological capability, the instrument can only be used for surface imaging instead of surface measuring. The strategies to overcome the nonlinearity of piezo transducer, in general, are of two types, open loop compensation and closed loop control. For an ideal $3 \mathrm{D}$ metrological system, all the motions in the six degrees of freedom should be strictly controlled. Any parasitic motion will influence the accuracy of measurement. In the common design of SPM, three rotational directions are assumed to be restricted by the translation structure itself, and only three linear motions need to be controlled. For the metrological SFM introduced above, the closed loop control method was adopted. The expansions of piezo actuators in $x, y$ and $z$ axes are monitored by three high sensitive capacitance sensors with which the nonlinearities along these axes can be reduced down to less than $0.2 \%$. For the first prototype of the $x-y$ scanning stage, the yaw errors were much larger than expected. The revised version of the stage is expected to give maximum yaw error of less than $10 \mu r d$ which is comparable with the linearities of the translations. Even so, further improvement is necessary for more precise applications. The $z$ translator (DPT) suffers from the same problem. Calibration shows that the pitching of $z$ DPT is $\pm 1.5^{\prime \prime}$ for whole expansion range $15 \mu \mathrm{m}$. A new version of the $\mathrm{DPT}$ is being developed in Queensgate Instruments Ltd. which will give a linear vertical displacument with insignificantly small motions in lateral directions.

The tube scanner which is most popularly used in SPM has unique merit in its fast dynamic response. The technique to monitor the motion of the tip, which is mounted at the bottom of the tube, in situ, is the key for the scanner to be used in metrological SPM. This will become realistic when the deflection shape of the tube can be controlled precisely. Ideally, an $S$ shape as mentioned in chapter 2 is expected, in this case the bottom of the tube scanner will have only lincar motions parallel to $x-y$ scanning plane, which can be easily monitored with linear sensors.

Many open loop compensation strategies have becn reported. One way to 
linearize the extension of the piezoelectric actuator is to provide a nonlinear compensatory drive voltage using a predetermined algorithm. The results do show an improvement in performance although present efforts are not adequate for the more demanding applications. Therefore, it is necessary to investigate a more sophisticated algorithmic model. To match the very complicated characteristics of piezo's expansion, many new modeling techniques such as neural network or fuzzy methods should be tried.

\subsection{What is measured}

Conventional surface measurement instruments using mechanical stylus or optical techniques were developed mainly for measuring surface geometric characteristic on various scales. Although the surface information is obtained from sensing some physical properties of the surface, in this scale range the geometric characteristic dominates the measurement results. For normal engineering materials, the differences of average effect of material properties on certain scale are insignificant. Therefore, there is little doubt about what is measured. As the results of the development of scanning probe microscopies which measure a range of physical and chemical properties of surface on the nanometer or even the atomic scale, the measurement object becomes obscure and difficult to define. In this case the surface geometric features go down to the same scale of inherent features of the physical or chemical properties of surface material. Both these features will always be included in the measurement results. This makes the interpretation of the results very difficult. To identify the geometric features from material property features, the first thing to be determined is the physical meaning of the surface "geometric" feature, or in other words, how to define the surface feature. From the engineering point of view, the geometric feature requirements are usually connected to the applications of some kinds of contacting activities, i.e. being more mechanical than physical. In this case, contact measurements tend to give a 
more practical definition of a surface geometric feature. Therefore, contact mode scanning force microscope measures the surface more geometrically. Because the measurement scales are extremely small, even for contact SFM the interactions between tip and surface are very complicated. The contact dynamic characteristics between various materials at such a micro regime have not been understood very well, which may have a direct influence on the fidelity of measurement. Contamination on surfaces is another problem. In our experiments, we also found that the interaction forces between tip and surface are less predictable. Fig.7.1 shows a situation of the interaction forces when a diamond tip is approaching and moving against a floating glass flat which is cleaned with Acetone. From the force curve it is clear that at near proximity of the surface exists an attractive force which pulls the tip jumping to the surface when it is about $50 \mathrm{~nm}$ from the surface. When the tip moves away from the surface, the effect of the attractive force can also be seen. Moreover, to achieve agreement results measured with var-

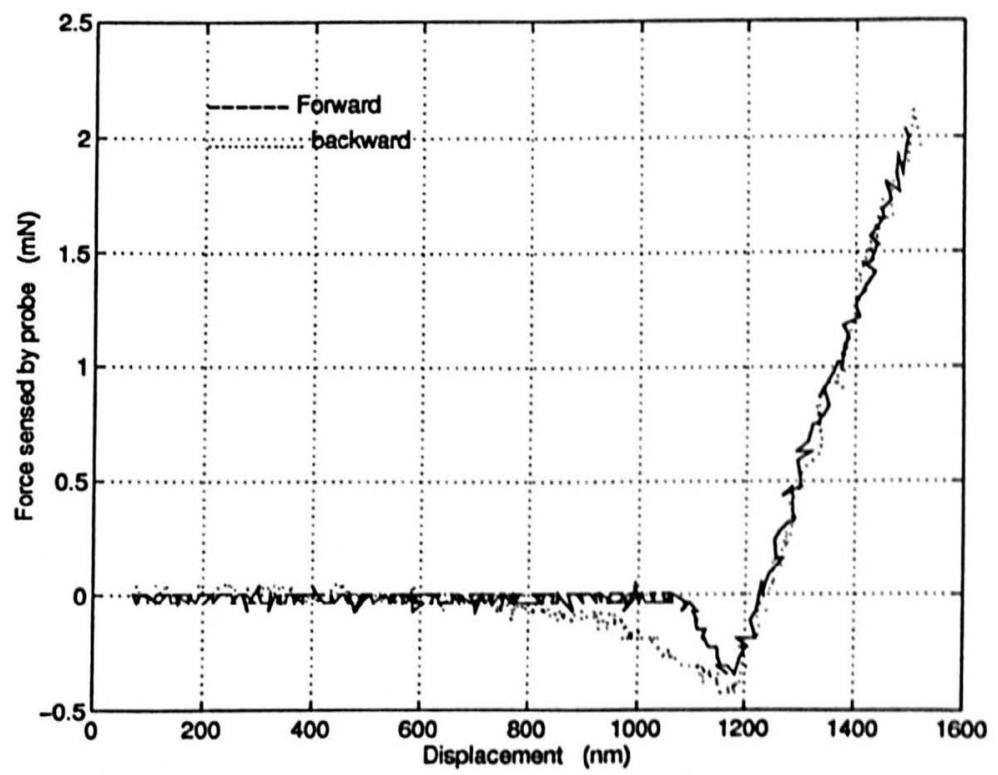

Figure 7.1: The interaction forces between a diamond tip and a floating glass cleaned with Acetone

ious instruments and by different people, a new standard for such measurement 
is necessary, which should at least include the factors of tip radius, $E$ moduluses of materials of both tip and specimen, contact forces, measuring speed, surface cleaning method and so on.

Many scanning probe microscopies were discussed in chapter 2. Each of them measures surface by sensing a particular property of surface material, so that it can meet a corresponding measuring requirement on surface property in some special aspects such as thermal, capacitance, magnetic, optical and chemical potential properties. However, for the present SPM techniques the measurement results always include the information of both surface geometric feature and material property feature as mentioned above. Techniques to separate these features are essential if more reliable results are to be achieved. If the surface geometric feature is defined as discussed above, a possible solution to the problem is to build a force probe combined SPM, i.e. combining other microscopies with a contact mode force probe. The force probe measures surface geometric feature and maintains the servo system following the surface, meanwhile, the other probes attached on the force probe may measure the surface physical or chemical properties which are interested.

\subsection{Further improvement on $\mathrm{X}-\mathrm{Y}$ stage}

$X-Y$ scanning stages have been increasingly used in SPM mainly because of its relative long scanning range and metrological capability. The design of the scanning stage (S220) jointly explored by University of Warwick and Queensgate Instruments Ltd. has been introduced in chapter 4 in detail. The motion translation system of the stage is formed by a group of flexure pivoted levers monolithically machined on an aluminium alloy block. The piezo actuators and position sensors are all embedded in the translation system. Although this design reaches the objectives very well for our application case, there are still some aspects of the design strategies which can be further improved for other applica- 
tions. In the aspect of performance accuracy, a symmetric translation mechanism is much better than the asymmetric translation mechanism. Because the antidriving forces in symmetric structure are distributed symmetricly, in theory, no yaw errors will be induced. The distortions of stage frame caused by driving forces is inevitable in the stage (S220) because the piezo actuators are embedded in the frame. In an author's resent design of $x$-y stage for scanning near-field optical microscope, these two problems have been properly dealt with and the stage is in manufacturing at Queensgate Instruments Ltd.. The configuration of the stage body is similar to the translation mechanism shown in Fig.4.11 (a), a typical symmetrical structure. The motion amplifying mechanisms are designed as separate components. The large piezo expansion forces are balanced within the amplifying mechanisms, Fig.7.2. The only forces acted on the stage are those

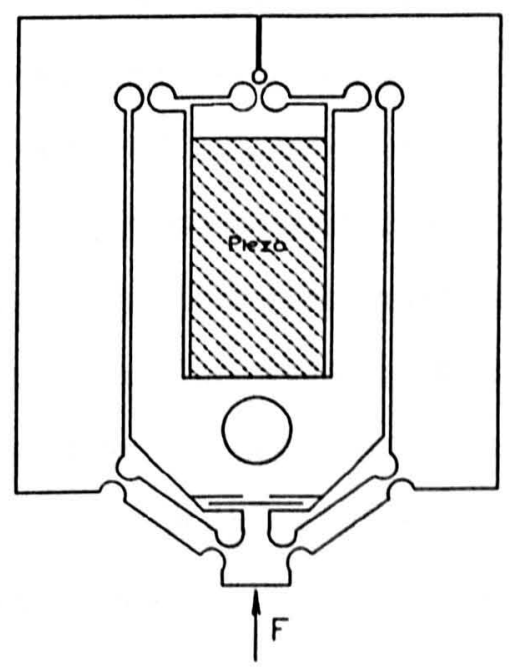

Figure 7.2: Schematic diagram of piezo expansion amplifier

required to match the stiffness of hinges around the platform, and they will be transferred to the platform through wobble pins. Very little distortions caused by driving forces are expected. Besides, to improve the fidelity of the performance damping effects have to be considered. Adding a magnetic force on the platform 
is one of strategies under investigation.

\subsection{Conclusion}

In this thesis, the development of a metrological scanning force microscope has been introduced with the aim of making an improvement on scanning probe microscopes in the aspect of instrumentation. The project is mainly composed of four parts: investigation of force probe, analysis of flexure translation mechanism and precision $x-y$ stage design, the manufacture and assessment of a metrological SFM, and surface measurement with the metrological SFM.

The force probe investigated in this thesis is a capacitance based constant force sensor. It has been successfully used in our scanning force microscope to obtain a subnanometer resolution. A dynamic model of the force probe, dominated by damping effect of squeezing air film between two electrodes, was built and experimentally verified. The results indicated that the model is viable for force probe parameter design and for prediction of its dynamic properties. The optimization of the probe design was discussed. It has been found that the force probe can be optimized in both deflection sensitivity and force sensitivity simultaneously. The former determines probe's capability of resolution, and the latter provides a low contact force between tip and surface. A proper sequence for parameter selection has been suggested based on the results of the theoretical analysis. The width of cantilever beam should be first chosen as narrow as possible. Then the minimum depth of air gap between two electrodes (mainly limited by damping force) is worked out. Finally, the length of the beam can be determined accordingly. Moreover, the investigation also shows that the force probe has an intrinsic good linearity in certain range, which makes it available to be used in both closed loop and open loop controls.

The flexure translation mechanism has unique characteristics for providing a smooth and accurate motion with a very simple structure. Two types of flexure 
spring components are usually used, leaf spring and notch hinge spring. The leaf spring system is more flexible for use when the deflection range is important. The simple hinge rectilinear mechanism has a higher accuracy of performance than that of simple leaf spring mechanism. The investigation shows that the accuracy of performance of the notch hinge mechanism is insensitive to manufacturing tolerances. The source of potentially significant errors are the driving force. The design strategies of the translation mechanisms are discussed in the design of precision $x-y$ stage. The FEA method is an effective tool for design of flexure translation mechanisms. Using this technique, the performances of device can be predicted for both static and dynamic characteristics.

A metrological scanning force microscope was built, which combines a constant force probe system, an $x-y$ scanning stage and a 3D coarse positioning mechanism into a metrological system. All the motions in three axes are monitored with capacitance NanoSensors and are in closed loop control. From calibration and preliminary performance evaluation, subnanometer resolution, stability and repeatability in all three axes are demonstrated in a normal laboratory environment. The contact force can be controlled over a range from $10^{-8}$ to $10^{-8} \mathrm{~N}$. Because of the application of the high sensitive position sensors in three axes, this SFM has a capability of not only imaging but also doing metrological measurement on precision engineering surfaces. The capability of directly providing areal information of surface characteristics is one of the advantage of SFM over stylus instruments. The low contact force makes it possible for use on soft materials without causing significant damage to the specimen. Some initial experimental results of surface measurement have been achieved showing its capability of measuring the specimens of various properties and features such as optical grating, silicon chip with integrated electronic elements, optical polished Zerodur flat and polymer film. It has also been successfully used for numerical analysis of surface roughness. The results from the SFM have a good agreement with that from Rank Taylor Hobson Nanostep and Bede GXR1 Reflectometer. 


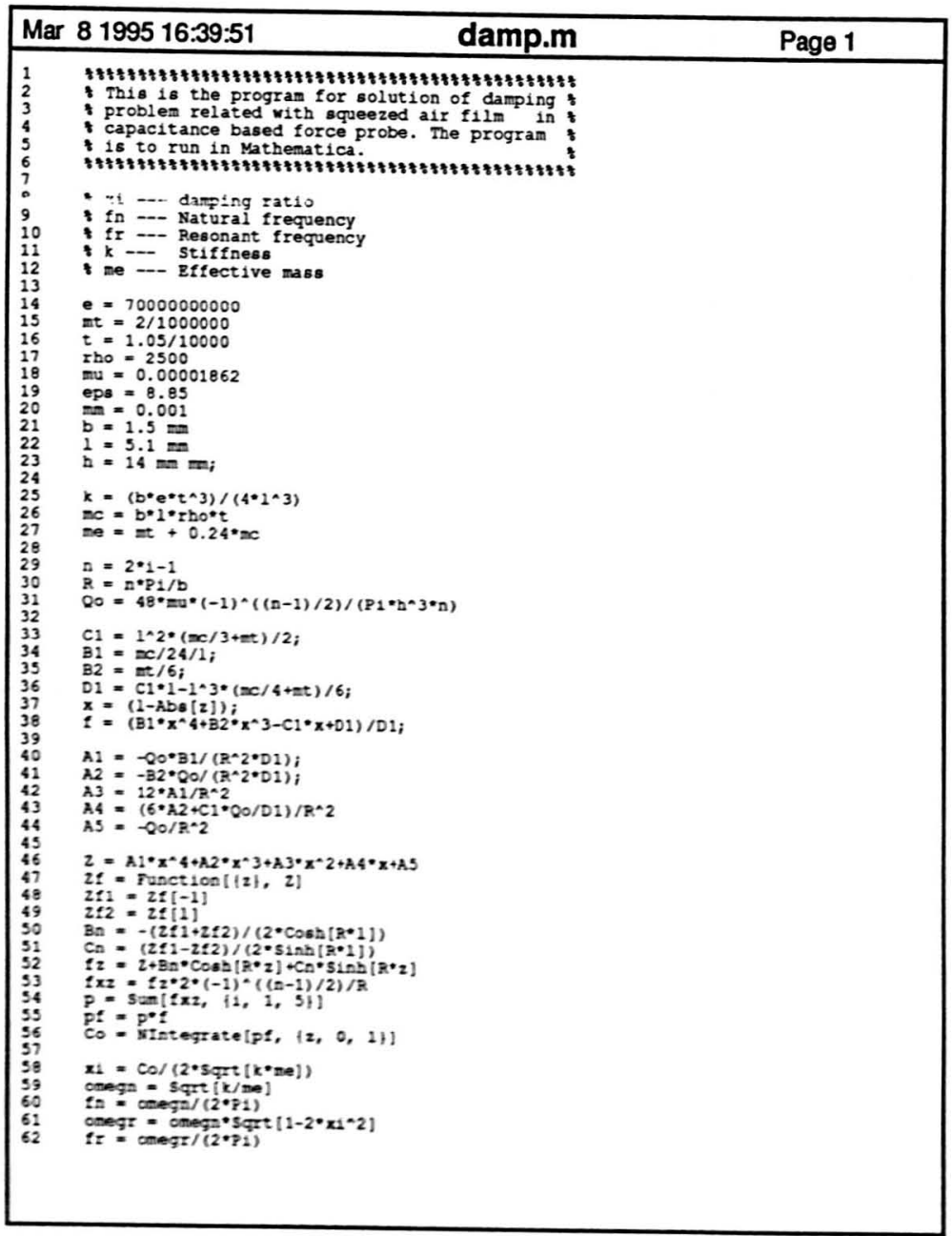

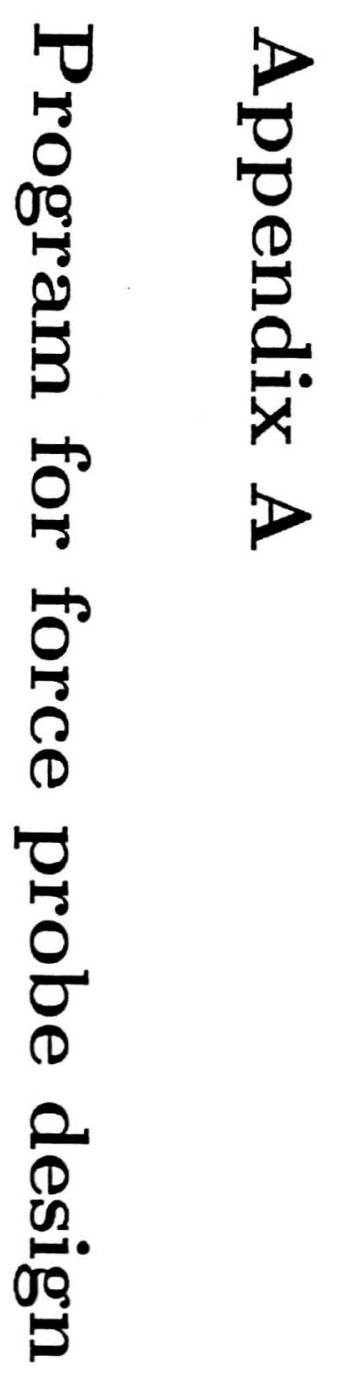




\begin{tabular}{|c|c|}
\hline Mar & damp_sen_g.m \\
\hline $\begin{array}{l}1 \\
2 \\
3 \\
4 \\
5 \\
6 \\
7 \\
8 \\
5 \\
10 \\
11 \\
12 \\
13 \\
14 \\
15 \\
16 \\
17 \\
18 \\
19 \\
20 \\
21 \\
22 \\
23 \\
24 \\
25 \\
26 \\
27 \\
28 \\
29 \\
30 \\
31 \\
32 \\
33 \\
34 \\
35 \\
36 \\
37 \\
39 \\
39 \\
40 \\
41 \\
41 \\
42 \\
43 \\
44 \\
45 \\
46 \\
47 \\
48 \\
49 \\
50 \\
51 \\
52 \\
53 \\
54 \\
55 \\
56 \\
57 \\
59 \\
59 \\
61 \\
61 \\
62 \\
63 \\
64 \\
65 \\
66 \\
\end{array}$ & 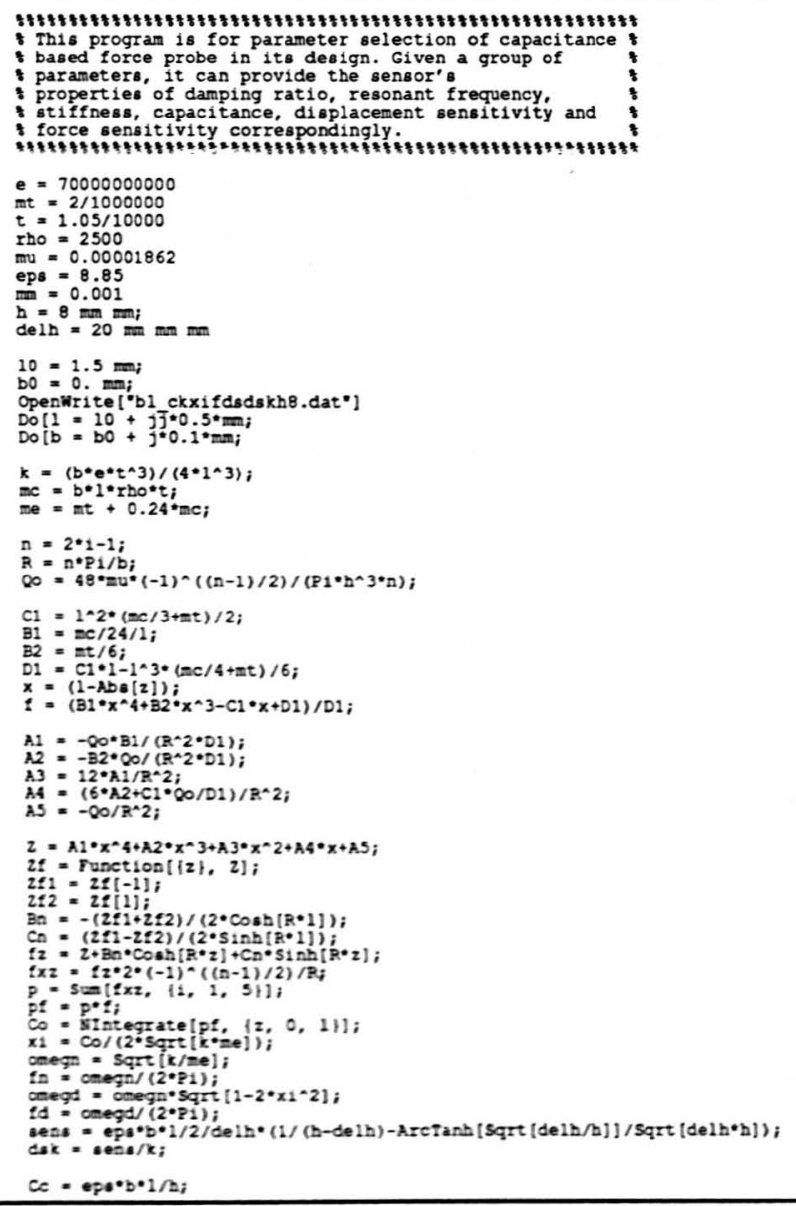 \\
\hline
\end{tabular}

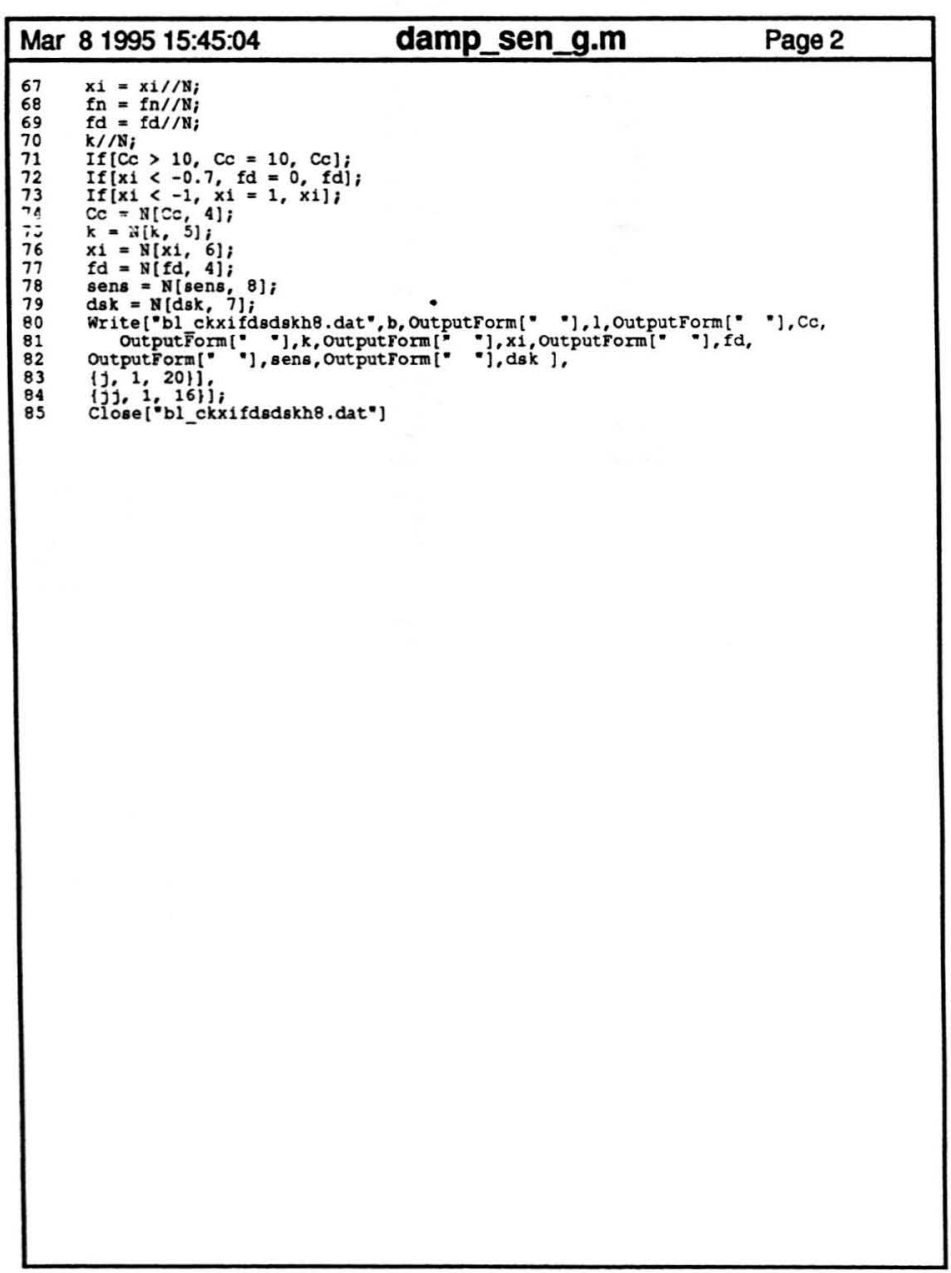




\section{Appendix B}

\section{Reaction force $R_{x}$}

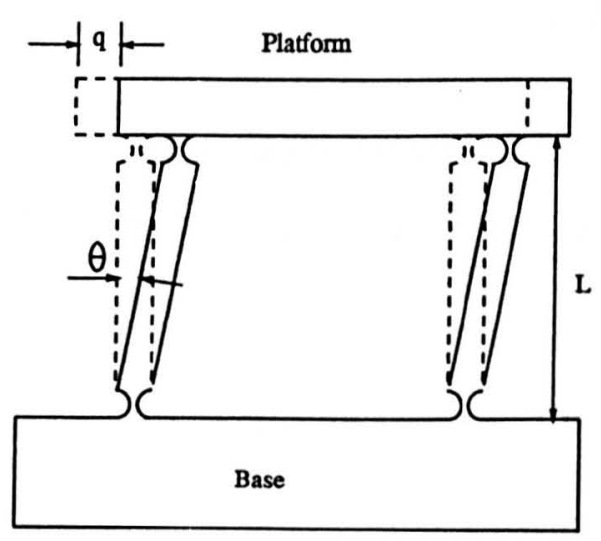

(a)

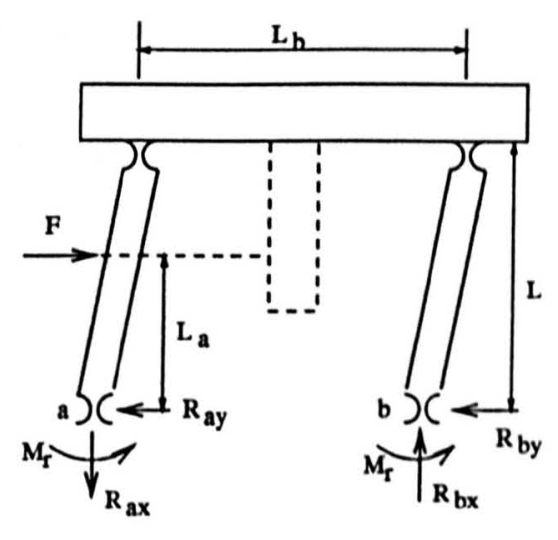

(b)

Figure B.1: Reaction force model of simple notch hinge rectilinear mechanism

Fig. B.1 shows a reaction force model of simple notch hinge rectilinear mechanism. It is clear that

$$
\begin{aligned}
R_{a y} & =R_{b y}=\frac{F}{2} \\
M_{r} & =k_{z} \theta
\end{aligned}
$$


The system elastic energy $V$ can be written as

$$
V=\frac{1}{2} k_{s} q^{2}=4\left(\frac{1}{2} k_{z} \theta^{2}\right)
$$

Where $k_{s}$ is the system stiffness and $k_{z}$ is single hinge stiffness. Then one can obtain

$$
k_{s}=\frac{4 k_{x}}{L^{2}}=\frac{F}{q}
$$

From Fig. B.1 (b), we can write out the relationship of the forces

$$
\begin{array}{r}
\sum M_{a}=0 \\
F L_{a}-2 M_{r}-R_{b x} L_{b}=0
\end{array}
$$

Then, by bringing equations B.2 \& B.4 into B.5, the axial reaction forces in the legs, $R_{x}\left(=R_{a x}=R_{b x}\right)$, can be worked out

$$
R_{x}=\frac{2 k_{z}\left(2 L_{a}-L\right)}{L^{2} L_{b}}
$$

